\title{
Genomic organization and spatio-temporal expression of the hemoglobin genes in European sea bass (Dicentrarchus labrax)
}

\author{
Cadiz L. ', Desmarais E. ${ }^{2}$, Servili Arianna ${ }^{1}$, Quazuguel Patrick ${ }^{1}$, Madec Lauriane ${ }^{1}$, \\ Huelvan Christine ${ }^{1}$, Andersen O. ${ }^{3,4}$, Zambonino Jose-Luis ${ }^{1}$, Mazurais David ${ }^{1,{ }^{*}}$
}

${ }^{1}$ Ifremer, Unité de Physiologie Fonctionnelle des Organismes MarinsPlouzané,France

2 Institut des Sciences de l'Evolution (UMR 5554), Université de Montpellier, CNRS-UM-IRD-

EPHEMontpellier,France

${ }^{3}$ NofimaÅs,Norway

${ }^{4}$ Department of Animal and Aquaculture SciencesNorwegian University of Life SciencesÅs,Norway

*Corresponding author : David Mazurais, email address : $\underline{\text { dmazurai@ifremer.fr }}$

\begin{abstract}
:
Hemoglobins $(\mathrm{Hb})$ play a critical role in satisfying the oxygen demand of vertebrate aerobic metabolism. The present study reports the characterization of the European sea bass (Dicentrarchus labrax) $\mathrm{Hb}$ genes, including genomic organization, phylogeny, and spatio-temporal gene expression. These $\mathrm{Hb}$ genes are divided into two unlinked clusters, the "MN" cluster containing eleven genes (five Hba genes named MN-Hba1-5 and six $\mathrm{Hb} \beta$ genes named $\mathrm{MN}-H b \beta 1-6)$ and the "LA" cluster consisting of three genes (two $\mathrm{Hba}$ genes named LA-Hba1-2 and one $\mathrm{Hb} \beta$ gene named $\mathrm{LA}-\mathrm{Hb} \beta 1$ ). Comparative analysis of $\mathrm{Hb}$ amino acid sequences indicates that most of the important amino acid residues involved in hemoglobin-oxygen binding, particularly in the Bohr and Root effects, are generally well conserved,

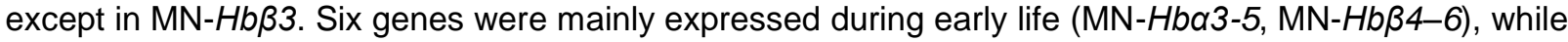
the others were predominantly expressed at juvenile-adult stages. Adult fish expressed $\mathrm{Hb}$ genes at high levels in the head kidney and spleen; the main organs involved in blood formation. The Hb genes expressed in non-hematopoietic organs (intestine, gills, heart, brain, and liver) may facilitate oxygen homeostasis or be involved in antimicrobial defense. Stage- and tissue-specific gene expression patterns, together with the sequence features of the different $\mathrm{Hb}$ proteins, suggest a broad range of roles in European sea bass.
\end{abstract}




\section{Introduction}

Teleost species inhabit contrasting marine and freshwater environments across the planet and face a wide variety of environmental conditions in terms of water temperature, salinity and dissolved oxygen concentration (Helfman et al. 1997; von der Heyden et al. 2015). Specifically, several fish species have offshore spawning and larval migration toward coastal areas. As a consequence, genetic adaptation or physiological acclimatization has been observed, especially to adjust oxygen demand (metabolism) and supply (oxygen uptake and transport) to environmental oxygen availability at the different stages of their life-cycle (Ishibashi et al. 2007; McKenzie et al. 2008). Several authors have suggested that the ability of fish to colonize a wide range of habitats has evolved together with the molecular and functional modulation of the hemoglobin system (Weber 2000; Verde et al. 2002; Marinakis et al. 2003; Verde et al. 2006; Verde et al. 2008). Hemoglobin ( $\mathrm{Hb})$ in vertebrates, including fish, consists of a tetrameric molecule composed of twin $\alpha$ and $\beta$ globin subunits $\left(\alpha_{2} \beta_{2}\right)$, each of which contain a heme group that can reversibly bind oxygen. While genes coding for $\alpha$ and $\beta$ globins in mammals are clustered on one chromosome, teleost $\alpha$ and $\beta$ globin genes ( $\mathrm{Hb} \alpha$ and $\mathrm{Hb} \beta$ genes) are grouped within two clusters labeled "MN" and "LA" located on two separate chromosomes resulting from the teleost-specific genome duplication (TGD) (Hardison 2008; Opazo et al. 2013). The cluster names are derived from their flanking genes: $\mathrm{N}$-methylpurine-DNA glycosylase $(\mathrm{mpg})$ and Nitrogen permease regulator-like 3 (nprl3) for cluster "MN", and Leucine carboxyl methyltransferase 1 (Icmt1) and Aquaporin-8 (aqp8) for cluster "LA". Both physical extent and gene content of the teleost globins have been altered by gene loss and by chromosomal or tandem duplications (Opazo et al. 2013) and the 
number of $\mathrm{Hb}$ genes varies from a single pseudogene in icefishes (Near et al. 2006) to 36 genes (including 9 pseudogenes) in Atlantic salmon (Quinn et al. 2010).

The multiplicity of $\mathrm{Hb}$ genes in many fish species is considered as an advantage that allows them to cope with a variable oxygen demand throughout ontogenic development as well as with temporal and spatial alterations in environmental oxygen availability (Perutz 1983; Rutjes et al. 2007). The strong adaptability has been shown to involve differential expression of $\mathrm{Hb}$ genes throughout the fish life cycle (Maruyama et al. 2004; Tiedke et al. 2011) and differential regulation under hypoxia or extreme conditions (Campo et al. 2008; Feng et al. 2014). Furthermore, the diversified $\mathrm{Hb}$ forms may exhibit different functional properties in terms of oxygen affinity and responsiveness to regulation by phosphates (ATP, GTP), temperature and pH (Bohr and Root effects) (Weber 1990).

European sea bass is an economically important farmed species with a natural distribution ranging in North Atlantic from the south of Norway $\left(60^{\circ} \mathrm{N}\right)$ to the west coast of Morocco $\left(30^{\circ} \mathrm{N}\right)$ and throughout the Mediterranean and Black Sea (Tortonese 1986; PerezRuzafa and Marcos 2014). The metabolic scope was shown to be susceptible to reduced oxygen availability and elevated temperatures, and hypoxia caused morphological changes and altered expression of genes coding for oxygen-dependent molecules in the gills of European sea bass (Claireaux and Lagardère 1999; Rinaldi et al. 2005). Multiple Hbs proteins have been documented by gel electrophoresis, and a partial globin with antimicrobial properties has been reported in European sea bass (Perez and Maclean 1976) (Terova et al. 2011). The recently published European sea bass genome sequence is a valuable source of information to investigate the globin genes (Tine et al. 2014). Starting from this genome assembly, the objectives of the present study were to clarify the organization of the genomic 
"MN" and "LA" clusters in European sea bass, in order to identify $\mathrm{Hb}$ genes and the predicted globin proteins expressed from embryonic to adult stages in this species.

\section{Materials and Methods}

\section{Genomic organization and structure analysis of $\mathrm{Hb}$ gene clusters}

The first genome assembly of European sea bass was produced based on high throughput sequencing and physical maps (Tine et al. 2014). This assembly was first annotated by homology at the protein level with five closely related published genomes and then by mapping of an annotated gene model constructed from RNA-Seq experiments. It contains 19 annotations for $\mathrm{Hb}$ genes localized in different linkage groups (LG), including two clusters, one composed of $3 \mathrm{Hb}$ genes in LG1B and the other composed of $8 \mathrm{Hb}$ genes in LG8. These clusters harbored the typical flanking genes of $\mathrm{Hb}$ clusters (i.e. Icmt1 and aqp8 for LG1B and $m p g$ and $n p r / 3$ for LG8), and probably corresponded to the LA and MN clusters, respectively (Hardison 2008). However, eight globin homolog genes were also detected in several unordered genomic contigs (UN), suggesting a potential incomplete or imperfect reconstitution of the genomic clusters in the published assembly. This is not surprising given the perfect homology that some exons of different $\mathrm{Hb}$ genes can display and the chromosomal clustering of these genes. In an attempt to improve the original assembly of the $\mathrm{Hb}$ cluster regions, especially with the goal of integrating the unplaced contigs containing $\mathrm{Hb}$ genes, we used an iterative process of assembly, based on sequential homology searches among long sequences of the plasmids used for building the initial physical map of the sea 
(http://seabass.mpipz.de/index.html?org=European+seabass\&db=dicLab1\&hgsid=3779). We first built a blast database containing the original Sanger sequences of the plasmids (500$1000 \mathrm{bp})$. We then blasted this database with the sequences of the flanking genes kank2 and aqp8 to retrieve homologous but overlapping plasmids to walk inward the $\mathrm{Hb}$ clusters. Once the first plasmids were merged into the gene sequences, we used this growing assembly as the query for another round of homology search, until we reached the other end of the cluster, i.e. the flanking gene on the other side of the $\mathrm{Hb}$ clusters in other species. The rationale for this approach was to benefit from the larger length of these sequences compared with those of NGS reads for resolving assembly ambiguities. Indeed, some parts of the $\mathrm{Hb}$ genes can be strictly identical sometimes over more than $200 \mathrm{bp}$ but never on longer stretch of sequence. Moreover, the plasmids contained genomic inserts up to several $\mathrm{Kb}$ long and were sequenced from both ends. We first built a (frame) scaffold of overlapping plasmid sequences by taking into account the pairing information between the two sequence ends of each cloned genomic fragment insert (sometime spanning a $5 \mathrm{~Kb}$ region of genome). We then plot the whole genome shotgun (WGS) short reads on that frame with BWA-MEM. The alignment of short reads was then manually curated with Geneious 9.1.7 over the two $\mathrm{Hb}$ clusters to remove inconsistently mapped pairs of reads, i.e. those without the two mates matching in this genomic region. Finally we reconstituted a consensus sequence based on the short reads and compare it to that obtained from the plasmids sequences only. This approach made it possible to reorganize the two globin clusters (LA and MN cluster sequences available in Supplementary file 1). Predicted exon-intron structures of genes were investigated using Genscan software (http://genes.mit.edu/GENSCAN.html) (Burge and Karlin 1997). The sequences of the $\mathrm{Hb}$ genes have been published in GenBank under accession numbers KX196178-KX196191. In order to validate the $\mathrm{Hb}$ gene succession 
in the MN Cluster, PCR primers were designed at different locations in this cluster and allowed to confirm the predicted amplicon sizes on genomic DNA (Supplementary file 2). Our LA cluster assembly was similar to that resulting from the automatic LG1B assembly published in the databases (http://seabass.mpipz.de/cgi-bin/hgGateway). Then based on the transcript sequences predicted from this new assembly we amplified cDNA that we cloned and sequenced (GenBank accession numbers: KY425658-KY425671), thereby confirming the biological existence of the new gene sequences we built.

\section{Sequence alignment and phylogenetic analysis}

$\mathrm{Hb} \alpha$ and $\mathrm{Hb} \beta$ amino acid sequences inferred by the translation of the European sea bass $\mathrm{Hb}$ cDNAs cloned in the present study were aligned using ClustalW along with sequences listed in a previous work (Opazo et al. 2013). Human and elasmobranch species were included as outgroup for alignment. To reconstruct the $\mathrm{Hb}$ phylogenies, the best model of evolution was assessed from this protein sequence alignment by means of the ProtTest 2.4 program (Abascal et al. 2005). Based on the Akaike information criterion (AIC), LG model was chosen as transition probability matrices (Le and Gascuel 2008), calculated as distance of amino acid substitution per site. We reconstructed $\mathrm{Hb}$ phylogeny including $\mathrm{Hb} \alpha$ and $\mathrm{Hb} \beta$ genes using maximum likelihood and Bayesian approaches. Maximum Likelihood phylogeny inference as implemented in MEGA v. 6 (Tamura et al. 2013) was used with 1000 replicates for the bootstrap test. The proportion of invariant sites was evaluated during the analysis, and the range of variation rate across site was determined by a gamma distribution with 4 categories. A Bayesian Markov Chain Monte Carlo (MCMC) analysis was performed using MrBayes software for testing evolutionary hypotheses in which the tree was weighted proportionally to their posterior probability. Two independent assays were performed for $\mathrm{Hb}$ 
alignment: LG was used as the substitution matrix, the site heterogeneity model was also a gamma distribution with 4 categories, and the number of MCMC replicates was $10^{6}$. The final results were summarized in the best tree after discarding the first $25 \%$ of them. All phylogenetic trees were viewed by FigTree v.1.4.3 software (http://tree.bio.ed.ac.uk/software/figtree/).

\section{Animals}

Eggs, juveniles ( 3 months post hatching) and adults (18 months post hatching) of European sea bass were obtained from a commercial hatchery (Aquastream, Ploemeur, France). Eggs were transferred to the wet laboratories in one 60-I incubator with open flow and aerated sea water kept at $15 \pm 0.4^{\circ} \mathrm{C}$. After hatching, larvae were fed daily with Artemia until the end of larval development (Zambonino-Infante et al. 1996). In order to investigate the gene expression pattern during embryonic and larval development, pools (in triplicate) of eggs and larvae between 20 and $50 \mathrm{mg}$ (containing five to several hundred individuals depending on developmental stage) were sampled at two embryonic (12 and $60 \mathrm{~h}$ post fertilization) and six larval stages $(5,13,19,26,33,45$ days post hatching). Juvenile ( $n=3$ per triplicate) and adult fishes $(n=3)$ were killed by lethal doses of anesthesia (Eugenol 0.05\%, Sigma-Aldrich Saint-Louis, Missouri, USA). Adult tissues (brain, gills, spleen, head kidney, heart, intestine and liver) were immediately dissected. Blood was quickly removed to reduce red blood cell contamination from tissues by rinsing with saline solution ( $\mathrm{NaCl} 0.01 \%)$. The pools of eggs and larvae as well as whole juvenile and adult tissues were transferred into microtubes containing RNAlater (Qiagen, Hilden, Germany) and placed at $4^{\circ} \mathrm{C}$ until total RNA extraction. Present work was performed in accordance with French and European policies and guidelines of the French Animal Care Committee (ACCF). No ethical approval for this study 
was necessary since fish were reared in optimal conditions until they were sacrificed. Fish were killed with overdose of Eugenol $(500 \mathrm{mg} / \mathrm{L})$ by prolonged immersion. Fish were left in the solution for $10 \mathrm{~min}$ at least following cessation of opercular movements. Fish were next decapitated to ensure brain death.

\section{RNA extraction and cDNA synthesis}

Total RNA for analysis of gene expression was extracted from the samples using Extract-all reagent (Eurobio; Courtaboeuf, Essonne, France) combined with the Zymo Direct-zol ${ }^{\mathrm{TM}}$ RNA MiniPrep Kit, following recommendations from the supplier. Genomic DNA was removed using the DNA-free Kit (MoBio Laboratories Inc.; Carlsbad, CA, USA). The quantity, purity and quality of RNA were assessed using a ND-1000 NanoDrop ${ }^{\circledR}$ spectrophotometer (Thermo Scientific Inc.; Waltham, MA, USA) and by electrophoresis using an Agilent Bioanalyser 2100 (Agilent Technologies Inc.; Santa Clara, CA, USA). Only samples with a RNA integrity number (RIN) greater than 7 were measured using real-time quantitative PCR (qPCR). RNA samples were stored at $-80^{\circ} \mathrm{C}$ until use.

Synthesis of cDNA was carried out using $0.5 \mu \mathrm{g}$ of DNase-treated total RNA with an iScript ${ }^{\mathrm{TM}}$ cDNA Synthesis kit (Bio-Rad Laboratories Inc.; Hercules, CA, USA). Total reaction was carried out in a final volume of $20 \mu \mathrm{L}$ containing $5 \mu \mathrm{L}$ of sample, $4 \mu \mathrm{L} 5 \mathrm{x}$ iScript $^{\mathrm{TM}}$ Reaction Mix, containing oligo(dT), random primers and RNaseA inhibitor, $1 \mu \mathrm{L}$ iScript $^{\mathrm{TM}}$ Reverse transcriptase and $10 \mu \mathrm{L}$ RNase/DNase free water. The cDNA synthesis reaction was incubated for $5 \mathrm{~min}$ at $25^{\circ} \mathrm{C}$ followed by $30 \mathrm{~min}$ at $42^{\circ} \mathrm{C}$ and terminated by incubation for 5 $\min$ at $85^{\circ} \mathrm{C}$ to inactivate the enzyme. Reverse transcription (RT) was performed using a Thermo-cycler TC-152 (Techne Barloworld Scientific; Stone, Staffordshire, UK). cDNA was stored at $-20^{\circ} \mathrm{C}$ until use. RT negative controls were performed on each sample. 


\section{Quantitative real-time RT-PCR analysis}

The analysis of globin gene expression at different developmental stages and in different tissues of European sea bass was carried out by quantitative RT-PCR using the primers given in table 1. Standard curves were estimated for each primer pair using serial dilutions (from $1 / 10$ to $1 / 270$ ) of a pool of cDNA. Efficiencies of qPCR for each pair of primers ranged from 95 to $100 \%$ with $\mathrm{R}^{2}>0.99$. Primers were designed using Primer 3 plus (http://www.bioinformatics.nl/cgi-bin/primer3plus/primer3plus.cgi).

Expression of the $\mathrm{Hb}$ genes was quantified using the iCycler MyiQ ${ }^{\mathrm{TM}}$ Single Color Real-Time PCR Detection System (Bio-Rad Laboratories Inc.). Each sample was run in triplicate in a final well volume of $15 \mu \mathrm{L}$ containing $5 \mu \mathrm{L}$ cDNA (1/30 dilution) and $10 \mu \mathrm{L}$ of reaction mix, composed of $0.5 \mu \mathrm{L}$ of each primer $(10 \mathrm{mM}), 1.5 \mu \mathrm{L}$ RNase/DNase free water, $7.5 \mu \mathrm{L} \mathrm{iQ}^{\mathrm{TM}}$ SYBR $^{\circledR}$ Green Supermix (Bio-Rad Laboratories Inc.) containing antibody-mediated hot-started iTaq DNA polymerase, dNTPs, $\mathrm{MgCl}_{2}, \mathrm{SYBR}^{\circledR}$ Green I dye, enhancers, stabilizers and fluorescein. Negative controls (non-template control) were systematically included in each plate. RT negative controls were also used as template in order to ensure the absence of residual DNA contamination. The qPCR profiles contained an initial activation step at $95^{\circ} \mathrm{C}$ for $2 \mathrm{~min}$, followed by 39 cycles: $5 \mathrm{~s}$ at $95^{\circ} \mathrm{C}$ and $20 \mathrm{~s}$ at annealing temperature $\left(60^{\circ} \mathrm{C}\right.$ for LA$H b \alpha 1, \mathrm{LA}-H b \alpha 2, \mathrm{LA}-H b \beta 1, \mathrm{MN}-H b \alpha 1, \mathrm{MN}-H b \alpha 2, \mathrm{MN}-H b \beta 1, \mathrm{MN}-H b \beta 2, \mathrm{MN}-H b \beta 3, \mathrm{MN}-H b \beta 4$ and reference genes; $62^{\circ} \mathrm{C}$ for $\mathrm{MN}-\mathrm{Hb} \beta 5$ and $\mathrm{MN}-\mathrm{Hb} \beta 6$; and $70^{\circ} \mathrm{C}$ for $\mathrm{MN}-\mathrm{Hb} \alpha 3, \mathrm{MN}-\mathrm{Hb} \alpha 4$ and $\mathrm{MN}-\mathrm{Hb} \alpha 5$ ). Annealing temperatures for each couple of primers were fixed by testing PCR condition on plasmids containing the different $\mathrm{Hb}$ cDNAs. Following this procedure, we determined $\mathrm{qPCR}$ condition allowing specific amplification of each $\mathrm{Hb}$ cDNA subtype. After 
the amplification phase, a melting curve was performed to confirm the amplification of a single product in each reaction.

For each sample, the corresponding Cq (Quantification cycle) value was determined automatically using "Gene Expression Module" of CFX Manager software (Bio-Rad Laboratories Inc.). Cq is the cycle number required to yield a detectable fluorescence signal. The relative quantity of messenger was normalized with the $\Delta \Delta \mathrm{Ct}$ method using the same CFX Manager software. Reference genes were used to correct for loading differences or other sampling variations present in each sample. The $18 \mathrm{~S}$ gene was chosen as reference gene for eggs, larvae and juvenile tissues, while elongation factor 1-alpha (ef1 $\alpha$ ) and 18S were used as reference genes in adult tissues. These reference genes were used since they did not show any significant variation of expression between samples (relative standard deviation $<5 \%$ among samples). Significant differences of expression between developmental stages were analyzed by one-way ANOVA using Tukey'HSD as post-hoc test $(p<0.05)$. Expression data were Log transformed to fit a normal distribution. Heat map was used to illustrate relative expression of each $\mathrm{Hb}$ gene during the fish development and among tissues. To this end, normalized data were subjected to hierarchical clustering (Distance metric selection: Pearson uncentered) and a heat map was generated using MeV software (Multi Experiment Viewer; http://www.tm4.org).

\section{Results}

\section{Genomic organization of globin gene clusters}

The MN and LA clusters in European sea bass were shown to contain a total of seven $\mathrm{Hb} \alpha$ and seven $\mathrm{Hb} \beta$ genes (Fig1). The $\mathrm{MN}$ cluster included five $\mathrm{Hb} \alpha$ genes annotated $\mathrm{MN}$ $H b \alpha 1$ to $\mathrm{MN}-\mathrm{Hb} \alpha 5$ and six $\mathrm{Hb} \beta$ genes annotated $\mathrm{MN}-H b \beta 1$ to $\mathrm{MN}-H b \beta 6$ following the suggested nomenclature (Opazo et al. 2013). The cluster consisted of about $42 \mathrm{~kb}$ from the 
first codon of $\mathrm{MN}-\mathrm{Hb} \alpha 5$ to the stop codon of $\mathrm{MN}-\mathrm{Hb} \beta 1$. Whereas stickleback (Gasterosteus aculeatus) $\mathrm{MN}-\mathrm{Hb} \alpha$ and $\beta$ genes were found on reverse and forward strands respectively, these genes were indifferently located on either strands in European sea bass (Fig1). A gene exhibiting high homology with the CCDC (Coiled-coil domain-containing protein) gene family was predicted on the reverse strand between $\mathrm{MN}-\mathrm{Hb} \beta 4$ and $\mathrm{MN}-\mathrm{Hb} \beta 5$.

The LA cluster included two $\mathrm{Hb} \alpha$ and one $\mathrm{Hb} \beta$ genes, annotated LA- $H b \alpha 1$, LA-Hb $\alpha 2$ and LA$H b \beta 1$ (Fig1). These three genes spanned a sequence length of about $14 \mathrm{~kb}$ from the first codon of LA-Hb $\alpha 2$ to the stop codon of LA-Hbo1. The Hbo genes of the LA cluster were encoded by one strand whereas $H b \beta 1$ was encoded by the reverse strand. LA-Hb $\alpha 1$ and LA$H b \beta 1$ were organized head to head whereas LA-Hb $\alpha 2$ and LA- $H b \beta 1$ were arranged tail to tail. The organization of the LA-cluster was conserved between the European sea bass and tilapia (Oreochromis niloticus).

\section{Structure of European sea bass hemoglobin genes}

The complete gene sequences and deduced coding sequences were confirmed by cDNA cloning. The European sea bass $\mathrm{Hb} \alpha$ and $\mathrm{Hb} \beta$ genes typically consist of three exons separated by two introns (Table 2). While all the $\mathrm{Hb} \beta$ genes coded for proteins of $147 \mathrm{aa}$, the size of the proteins encoded by $\mathrm{Hb} \alpha$ genes ranged between 141 and 144 aa. 


\section{Analysis of putative key residues in European sea bass $\mathrm{Hb}$}

The histidine (His) residues deemed to be implicated in oxygen binding were conserved in the predicted $\mathrm{Hb} \alpha$ and $\mathrm{Hb} \beta$ globins (Figs2 and 3). The His residue at position 122 of $\mathrm{Hb} \alpha$ globins seems to be involved in the Bohr effect (Bellelli and Brunori 2011) and was found in all $\mathrm{Hb} \alpha$ sequences of European sea bass. Similarly, the C-terminal His residue of $\mathrm{Hb} \beta$ responsible for the Bohr effect was also present in all $\mathrm{Hb} \beta$ sequences, except for $\mathrm{MN}-\mathrm{Hb} \beta 3$ where it is substituted by a Phe residue. European sea bass $\mathrm{Hb} \alpha$ and $\mathrm{Hb} \beta$ genes possessed all the putative residues responsible for the Root effect, such as Val2 $\beta$, Ser $2 \alpha$, Trp4 $\beta$, Ser90 $\beta$, Ser94 $\beta$, Glu95 $\beta$, Asp95 $\alpha$, Asp101 $\beta$, Glu140 $\alpha$, Arg144 $\beta$, Gln145 $\beta$, Tyr146 $\beta$ and His147 $\beta$ (Bonaventura et al. 2004), except $\mathrm{MN}-\mathrm{Hb} \beta 3$ which was devoid of Ser94 $\beta$ and $\operatorname{Arg} 144 \beta$.

\section{Phylogenetic analysis of European sea bass $\mathrm{Hb}$}

Phylogenetic analysis was performed based on the deduced $\mathrm{Hb} \alpha$ and $\mathrm{Hb} \beta$ amino acid sequences of the European sea bass and other teleost and non-teleost species (Supplementary file 3). The data set was used to calculate the best $\mathrm{Hb}$ tree using maximum likelihood (ML) approach and the Bayesian MCMC methods. Since the two methods led to similar phylogenies, we only present the tree obtained with the Bayesian approach (Figs4 and 5) where the nodes appeared to be more robust, i.e. posterior probabilities above 0.5 . The phylogenetic analysis shed light on the ortho- versus paralogy relationships of the $D$. labrax genes with those of other species. The tree allowed to group Hbo in many clades, two 
of them composed with genes from the LA cluster. $\mathrm{Hb} \beta$ are grouped in four major clades including three clades related to the MN cluster and one related to the LA cluster.

While each $\mathrm{Hb} \beta$ clade included at least one $\mathrm{Hb} \beta$ globin from European sea bass, only four of the five $\mathrm{Hb} \alpha$ clades contained one or more European sea bass $\mathrm{Hb} \alpha$ globins. Analysis of the MN clades revealed that European sea bass $\mathrm{MN}-\mathrm{Hb} \alpha 3-5$ on one hand and $\mathrm{MN}-\mathrm{Hb} \beta 2, \mathrm{MN}-$ $\mathrm{Hb} \beta 4-6$ on the other clustered together in the $\mathrm{MN}-\mathrm{Hb} \alpha$ and $\mathrm{MN}-\mathrm{Hb} \beta$ clades, respectively, that is consistent with the high coding sequence similarities (Supplementary file 4).

\section{Expression pattern of $\mathrm{Hb}$ genes during developmental stages}

The temporal expression patterns of the $\mathrm{Hb}$ genes varied considerably, but some common features can be noted (Fig6, table 3). During embryogenesis (12 h and $60 \mathrm{~h}$ post-fertilization; hpf) relative expression levels of both $\mathrm{Hb} \alpha$ and $\mathrm{Hb} \beta$ genes were very low or not detected

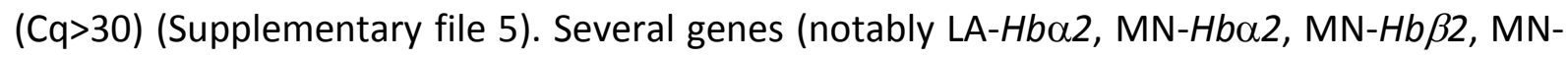
$H b \alpha 3, \mathrm{MN}-\mathrm{Hb} \beta 5, \mathrm{MN}-\mathrm{Hb} \beta 4$ and $\mathrm{MN}-\mathrm{Hb} \alpha 5)$ exhibited a substantial increase in expression between $60 \mathrm{hpf}$ and 5 days post hatching (dph). The expression levels of several genes (LA$H b \alpha 2, \mathrm{LA}-H b \beta 1, \mathrm{MN}-H b \beta 1, \mathrm{MN}-H b \alpha 2, \mathrm{MN}-H b \beta 4, \mathrm{MN}-H b \alpha 5, \mathrm{MN}-\mathrm{Hb} \alpha 4$ and $\mathrm{MN}-\mathrm{Hb} \beta 6$ ) increased exponentially between 5 and $45 \mathrm{dph}$, with a key step between $26 \mathrm{dph}$ and $45 \mathrm{dph}$. After $45 \mathrm{dph}$, expression increased substantially for some genes (LA- $H b \beta 1, \mathrm{MN}-H b \alpha 2$, LA$H b \alpha 1, \mathrm{MN}-H b \alpha 1, \mathrm{MN}-H b \beta 2$ and $\mathrm{MN}-H b \alpha 2)$ during the juvenile stage, while it dropped significantly for others ( $\mathrm{MN}-\mathrm{Hb} \beta 4, \mathrm{MN}-\mathrm{Hb} \alpha 5, \mathrm{MN}-\mathrm{Hb} \alpha 4$ and $\mathrm{MN}-\mathrm{Hb} \beta 6$ genes). $\mathrm{MN}-\mathrm{Hb} \beta 3$ was very weakly expressed $(\mathrm{Cq}>35)$ at all the developmental stages investigated. 


\section{Tissue expression pattern of $\mathrm{Hb}$ genes}

Based on the order of $\mathrm{Cq}$ value magnitude, $\mathrm{MN}-\mathrm{Hb} \alpha 1$ and $\mathrm{MN}-\mathrm{Hb} \beta 1$ exhibited the highest expression levels in the adult fish, irrespective of the tissue considered (Supplementary file 6). In contrast, genes $\mathrm{MN}-H b \alpha 3, \mathrm{MN}-H b \alpha 4$ and $\mathrm{MN}-H b \alpha 5$, as well as $\mathrm{MN}-H b \beta 4, \mathrm{MN}-H b \beta 5$ and $\mathrm{MN}-\mathrm{Hb} \beta 6$, were expressed at very low levels in the different adult tissues investigated. $\mathrm{MN}-H b \alpha 1, \mathrm{MN}-H b \beta 1$ and $\mathrm{MN}-H b \beta 3$ showed the highest expression levels in the head kidney and lesser levels in the spleen (Fig7, Table 4). $\mathrm{MN}-\mathrm{Hb} \alpha 2$ and $\mathrm{MN}-\mathrm{Hb} \beta 2$ genes showed the highest transcript levels in spleen. Several $\mathrm{Hb}$ genes were expressed at low levels in other non-hematopoietic tissues such as brain, heart, intestine and gills.

\section{Discussion}

In order to gain knowledge about $\mathrm{Hb}$ genes in the European sea bass, we assembled and confirm by transcript sequencing fourteen $\mathrm{Hb}$ genes that we assigned onto two genomic LA and MN clusters localized respectively on the linking group (LG) 1B (Sequence ID: emb|CBXY010008361.1|) and LG 8 (Sequence IDs: emb|CBXY010016043.1|emb|CBXY010016046.1|) of the first assembly of the European sea bass genome (Tine et al. 2014).

Three genes ( $2 \mathrm{Hb} \alpha$ and $1 \mathrm{Hb} \beta$ ) were located adjacent to the conserved aqp8 and lcmt1 genes of the LA cluster in teleosts (Opazo et al. 2013). As observed in platyfish, medaka and tilapia, the two $\alpha$-globin genes were separated by the $\beta$-globin gene located on the opposite strand (Opazo et al. 2013). The similar organization of this cluster suggests that the LA $\alpha-$ 
and $\beta$-globin genes of European sea bass are 1:1 orthologs with those in platyfish, medaka and tilapia, in agreement with the phylogenetic analysis which grouped the three LA genes within the respective LA-clades of these species. The LA clades also included orthologous globin genes from green spotted puffer and fugu, wherein these genes have been reversed in the LA cluster. We noted that the ortholog of sea bass LA-Hb $\beta 1$ seems to have been lost in stickleback, which yet exhibits high overall orthologous sequence homologies with European sea bass (Tine et al. 2014). Our data also revealed that eleven globin genes (five $\mathrm{Hb} \alpha$ and six $\mathrm{Hb} \beta$ ) were flanked by $n p r l 3$ and kank2 genes similar to the $\mathrm{MN}$ cluster in teleosts. Intriguingly, a $C C D C 106-$ like gene was found inserted between $\mathrm{MN}-H b \beta 4$ and $\mathrm{MN}-H b \beta 5$, and the specific functional role of this potential p53-interacting partner (Zhou et al. 2010) warrants further studies. It is accepted that distinct organization between teleost species in the LA and, especially, MN clusters results from lineage-specific changes in gene content via repeated duplication and deletion events (Opazo et al. 2013). Our phylogenetic analysis suggests that two gene groups ( $\mathrm{MN}-\mathrm{Hb} \alpha 3, \mathrm{MN}-\mathrm{Hb} \alpha 4, \mathrm{MN}-\mathrm{Hb} \alpha 5$ and $\mathrm{MN}-\mathrm{Hb} \beta 2, \mathrm{MN}-\mathrm{Hb} \beta 4$, $\mathrm{MN}-H b \beta 5, \mathrm{MN}-H b \beta 6$ ) each consist of close paralogous copies and are probably the results of recent duplication events that occurred in the Moronidae lineage. In comparison, $\mathrm{MN}-\mathrm{Hb} \alpha 4$ and $\mathrm{MN}-\mathrm{Hb} \beta 2$ branch deeper in the phylogenetic trees, suggesting that they might be ancestral with regard to their respective paralog copies located at the tips of their branch. In any case, from an evolutionary point of view, the $\mathrm{MN}$ cluster seems to be largely more dynamic than the LA one, which was suggested to have been lost in Atlantic salmon (Quinn et al. 2010).

The LA-Hb genes of European sea bass did not show expression patterns specific to the early life stage, contrary to the embryonic $\mathrm{Hb}$ genes in medaka, zebrafish and tilapia (Maruyama 
et al. 2004; Tiedke et al. 2011; Opazo et al. 2013). However, the three MN-Hba3-5 genes and three $\mathrm{MN}-\mathrm{Hb} \beta 4-6$ genes exhibited high larval expression compared to juvenile and adult levels. Intriguingly, the expression patterns throughout development were similar in the closely linked $\mathrm{MN}-\mathrm{Hb} \alpha 4, \mathrm{MN}-\mathrm{Hb} \alpha 5$ and $\mathrm{MN}-\mathrm{Hb} \beta 6$ on one hand and in the neighbour genes $\mathrm{MN}-\mathrm{Hb} \alpha 3$ and $\mathrm{MN}-\mathrm{Hb} \beta 5$ on the other hand, suggesting that these $\mathrm{Hb} \alpha$ and $\mathrm{Hb} \beta$ genes likely display common elements or/and mechanisms of regulation. With the new assembly we built, it will be now possible to investigate further the existence of such regulation elements. The common feature of the $\mathrm{Hb}$ genes significantly expressed just after hatching, was the increased expression from $60 \mathrm{hpf}$ to $5 \mathrm{dph}$. This data suggests that around the $60 \mathrm{hpf}$ stage oxygen supply by simple diffusion throughout the embryonic tissues may be sufficient to fulfill physiological requirements in European sea bass. The absence of significant $\mathrm{Hb}$ gene expression in fertilized eggs of sea bass was consistent with data obtained in another seawater species, the Atlantic cod, but contrasted with the expression profiles of embryonic genes observed in medaka and zebrafish (Maruyama et al. 2004; Wetten et al. 2010; Tiedke et al. 2011). It has been shown that the expression of embryonic $\mathrm{Hb}$ genes in zebrafish starts with primitive erythropoiesis, which takes place in the intermediate cell mass around $15 \mathrm{hpf}$ (Kulkeaw and Sugiyama 2012). The stage at which intermediate cell mass develops is not known in European sea bass; however, our data suggested that primitive erythropoiesis resulting in matured erythrocytes that are replete with $\mathrm{Hb}$ occurs after $60 \mathrm{hpf}$. Moreover, European sea bass $\mathrm{Hb}$ genes that did not show high mRNA levels during the first stage of larval development (LA-Hb $\alpha 1, \mathrm{LA}-H b \alpha 2, \mathrm{MN}-H b \alpha 1$, $\mathrm{MN}-H b \alpha 2, \mathrm{LA}-H b \beta 1, \mathrm{MN}-H b \beta 1, \mathrm{MN}-H b \beta 2$ and $\mathrm{MN}-H b \beta 3)$ showed a progressive increase in gene expression from $26 \mathrm{dph}$ to juvenile/adult stage. Most of these "late expressed" $\mathrm{Hb}$ genes exhibited a significant rise in expression between 26 and $33 \mathrm{dph}$. Interestingly, this 
developmental window was previously associated with a switch of global transcriptomic profile related to a key physiological step in the development of European sea bass larvae (Darias et al. 2008). This increase in $\mathrm{Hb}$ gene expression was likely related to the transition from primitive to definitive erythropoiesis, which is associated with the emergence of the hematopoietic function in the kidney and spleen during larval development of teleosts (Brownlie and Zon 1999). Indeed, erythropoietic activity has been detected in the spleen of European sea bass before $40 \mathrm{dph}$ (Quesada et al. 1994). In zebrafish, the switch from primitive to definitive erythropoiesis has been shown to start around $10 \mathrm{dph}$ (Tiedke et al. 2011). The earlier expression of $\mathrm{Hb}$ embryonic genes, as well as the earlier period of transition between embryonic and adult $\mathrm{Hb}$ expression observed in zebrafish, can be explained by faster developmental processes in this species.

The present investigation revealed that head kidney and, to a lesser extent, spleen are the major tissues in which most of European sea bass $\mathrm{Hb}$ genes were expressed at adult stage. Consistently, spleen, kidney (mesonephros) and head kidney (pronephros) are the sites of erythropoiesis in European sea bass (Esteban et al. 1989; Quesada et al. 1994) and other teleosts species, such as catfish (Ictalurus punctatus) (Feng et al. 2014) and euryhaline flounder (Platichthys flesus) (Lu et al. 2011). MN-Hb $\alpha 1, \mathrm{MN}-H b \beta 1$ and to a lesser extent LA$H b \alpha 2, \mathrm{MN}-H b \beta 3, \mathrm{MN}-H b \alpha 2$ and $\mathrm{MN}-H b \beta 2$ were the most predominantly expressed genes at the adult stage. The expression of three different $\mathrm{Hb} \alpha$ and three different $\mathrm{Hb} \beta$ genes in adult European sea bass is consistent with the identification by gel electrophoresis of five major $\mathrm{Hb}$ tetramers which likely comprise different combinations of these subunits (Perez and Maclean 1976). Most of the European sea bass Hb genes exhibited moderate expression level in non-hematopoietic tissues such as brain, heart, liver, intestine and gill. Consistently, 
expression of $\mathrm{Hb}$ in several vertebrates has been found in many non-erythroid cells (Saha et al. 2014), in which it may facilitate tissue oxygen transport or increase cellular oxygenation (Nishi et al. 2008; Biagioli et al. 2009; Tezel et al. 2010). In channel catfish, the expression of $\mathrm{Hb}$ genes in the intestine is associated with the respiratory function of this tissue (Feng et al. 2014). Moreover, antimicrobial activity has also been attributed to $\mathrm{Hb} \beta$ in gills and skin of channel catfish exposed to parasite (Ullal et al. 2008). Such antimicrobial activity is suggested to be also associated with the increase of $\mathrm{Hb} \beta$-like (highly similar to the present $\mathrm{MN}-H b \beta 1 \mathrm{cDNA}$ sequence) expression in the epithelium of European sea bass submitted to an acute stress (Terova et al. 2011).

Compared to mammals, the functional property of fish Hbs known as Root effect is a decrease in both Hb-oxygen affinity and cooperativity at low blood $\mathrm{pH}$. Even if the Root effect cannot be based solely on the presence or absence of residues, Asp95 $\alpha$ and Asp101 $\beta$, that are considered as the minimal structural requirement for the Root effect (Mazzarella et al. 2006), were conserved in the different European sea bass $\mathrm{Hb}$ genes. Moreover, Ser94 $\beta$, $\operatorname{Arg} 144 \beta$ and His147 $\beta$ also known to be important amino acid residues involved in Hb-oxygen binding and particularly in the Root effect (Mylvaganam et al. 1996; Bonaventura et al. 2004) were conserved in the different European sea bass $\mathrm{Hb} \beta$ genes except for $\mathrm{MN}-H b \beta 3$. The substitution of key functional residues are partially found in $H b \beta 1, H b \beta 4$ and $H b \beta 5$ of catfish (Feng et al. 2014) and in trout $\mathrm{Hb} I$ (Gabbianelli et al. 2004). Functional characterization of the European sea bass $\mathrm{Hb}$ proteins related to water temperature, oxygen affinity and $\mathrm{pH}$ sensitivity will be necessary to determine potential specific functional properties. 


\section{Conclusion}

Even though the composition the $\mathrm{Hb}$ tetramers in European sea bass $\mathrm{Hb}$ and their functional properties are not known, present data support the evidence that European sea bass is capable of producing different types of $\mathrm{Hb}$ with potential different functional properties over their life cycle. The positive relation between the diversity of $\mathrm{Hb}$ genes expressed in fish species and the diversity of their living environments suggests an important role played by these oxygen binding proteins in the ability to cope with environmental constraints. This appears to be particularly relevant in the European sea bass, which inhabits coastal nurseries with large fluctuations in environmental conditions.

\section{Acknowledgments}

The first author was supported by a joint Ifremer-Région Bretagne doctoral grant and bythe "Laboratoire d'Excellence" LabexMER (ANR-10-LABX-19). The authors are very grateful to Laure Quintric and Fanny Marquer (Cellule bioinformatique, Centre Ifremer Bretagne) for bioinformatic assistance, to Dorothée Vincent for correcting the manuscript and to Helen Mc Combie for correcting the English.

\section{Compliance with Ethical Standards:}

Conflict of Interest: The authors declare that they have no conflict of interest.

Ethical approval : All applicable international, national, and/or institutional guidelines for the care and use of animals were followed. 


\section{References}

Abascal F, Zardoya R, Posada D (2005) ProtTest: selection of best-fit models of protein evolution. Bioinformatics 21: 2104-2105

Bellelli A, Brunori M (2011) Hemoglobin allostery: Variations on the theme. Biochimica et Biophysica Acta (BBA) - Bioenergetics 1807: 1262-1272

Biagioli M, Pinto M, Cesselli D, Zaninello M, Lazarevic D, Roncaglia P, Simone R, Vlachouli C, Plessy $C$, Bertin N, Beltrami A, Kobayashi K, Gallo V, Santoro C, Ferrer I, Rivella S, Beltrami CA, Carninci $P$, Raviola E, Gustincich $S$ (2009) Unexpected expression of alpha- and beta-globin in mesencephalic dopaminergic neurons and glial cells. Proc Natl Acad Sci U S A 106: 1545415459

Bonaventura C, Crumbliss AL, Weber RE (2004) New insights into the proton-dependent oxygen affinity of Root effect haemoglobins. Acta Physiol Scand 182: 245-258

Brownlie A, Zon L (1999) The Zebrafish as a Model System for the Study of Hematopoiesis: Zebrafish mutants point the way to novel genes involved in the generation of vertebrate blood cells. BioScience 49: 382-392

Burge C, Karlin S (1997) Prediction of complete gene structures in human genomic DNA. J Mol Biol 268: 78-94

Claireaux G, Lagardère JP (1999) Influence of temperature, oxygen and salinity on the metabolism of the European sea bass. Journal of Sea Research 42: 157-168

Darias MJ, Zambonino-Infante JL, Hugot K, Cahu CL, Mazurais D (2008) Gene expression patterns during the larval development of European sea bass (dicentrarchus labrax) by microarray analysis. Mar Biotechnol (NY) 10: 416-428

Esteban MA, Meseguer J, Garcia Ayala A, Agulleiro B (1989) Erythropoiesis and thrombopoiesis in the head-kidney of the sea bass (Dicentrarchus labrax L.): an ultrastructural study. Arch Histol Cytol 52: 407-419

Feng J, Liu S, Wang X, Wang R, Zhang J, Jiang Y, Li C, Kaltenboeck L, Li J, Liu Z (2014) Channel catfish hemoglobin genes: identification, phylogenetic and syntenic analysis, and specific induction in response to heat stress. Comp Biochem Physiol Part D Genomics Proteomics 9: 11-22

Gabbianelli R, Zolese G, Bertoli E, Falcioni G (2004) Correlation between functional and structural changes of reduced and oxidized trout hemoglobins I and IV at different pHs. A circular dichroism study. Eur J Biochem 271: 1971-1979

Hardison RC (2008) Globin genes on the move. J Biol 7: 35

Helfman G, Collete B, Facey D (1997) The Diversity of Fishes Malden, Mass.

Ishibashi Y, Kotaki T, Yamada Y, Ohta $H$ (2007) Ontogenic changes in tolerance to hypoxia and energy metabolism of larval and juvenile Japanese flounder Paralichthys olivaceus. Journal of Experimental Marine Biology and Ecology 352: 42-49 
Kulkeaw K, Sugiyama D (2012) Zebrafish erythropoiesis and the utility of fish as models of anemia. Stem Cell Res Ther 3: 55

Le SQ, Gascuel O (2008) An improved general amino acid replacement matrix. Mol Biol Evol 25: 13071320

Lu W, Mayolle A, Cui G, Luo L, Balment RJ (2011) Molecular Characterization and Expression of alphaGlobin and beta-Globin Genes in the Euryhaline Flounder (Platichthys flesus). Evid Based Complement Alternat Med 2011: 965153

Marinakis $P$, Tamburrini $M$, Carratore V, di Prisco $G$ (2003) Unique features of the hemoglobin system of the Antarctic notothenioid fish Gobionotothen gibberifrons. Eur J Biochem 270: 3981-3987

Maruyama K, Yasumasu S, Naruse K, Mitani H, Shima A, luchi I (2004) Genomic organization and developmental expression of globin genes in the teleost Oryzias latipes. Gene 335: 89-100

Mazzarella L, Bonomi G, Lubrano MC, Merlino A, Riccio A, Vergara A, Vitagliano L, Verde C, di Prisco G (2006) Minimal structural requirements for root effect: crystal structure of the cathodic hemoglobin isolated from the antarctic fish Trematomus newnesi. Proteins 62: 316-321

McKenzie DJ, Lund I, Pedersen PB (2008) Essential fatty acids influence metabolic rate and tolerance of hypoxia in Dover sole (Solea solea) larvae and juveniles. Marine Biology 154: 1041-1051

Mylvaganam SE, Bonaventura C, Bonaventura J, Getzoff ED (1996) Structural basis for the Root effect in haemoglobin. Nat Struct Mol Biol 3: 275-283

Near TJ, Parker SK, Detrich HW, 3rd (2006) A genomic fossil reveals key steps in hemoglobin loss by the antarctic icefishes. Mol Biol Evol 23: 2008-2016

Nishi $H$, Inagi $R$, Kato $H$, Tanemoto $M$, Kojima I, Son D, Fujita T, Nangaku M (2008) Hemoglobin is expressed by mesangial cells and reduces oxidant stress. J Am Soc Nephrol 19: 1500-1508

Opazo JC, Butts GT, Nery MF, Storz JF, Hoffmann FG (2013) Whole-genome duplication and the functional diversification of teleost fish hemoglobins. Mol Biol Evol 30: 140-153

Perez-Ruzafa A, Marcos C (2014) Ecology and Distribution of Dicentrarchus labrax (Linnaeus 1758). In: Sanchez Vazquey J, Munoz-Cueto J (eds) Biology of European Sea Bass. CRC Press, Boca Raton, FL, U.S.A., pp 3-33

Perez JE, Maclean N (1976) Multiple globins and haemoglobins in the bass, Dicentrarchus labrax (L.) (Serranidae: Teleostei). Journal of Fish Biology 8: 413-417

Perutz MF (1983) Species adaptation in a protein molecule. Mol Biol Evol 1: 1-28

Quesada J, Villena MI, Navarro V (1994) Ontogeny of the sea bass spleen (Dicentrarchus labrax): A light and electron microscopic study. Journal of Morphology 221: 161-176

Quinn NL, Boroevich KA, Lubieniecki KP, Chow W, Davidson EA, Phillips RB, Koop BF, Davidson WS (2010) Genomic organization and evolution of the Atlantic salmon hemoglobin repertoire. BMC Genomics 11: 539 
Rinaldi L, Basso P, Tettamanti G, Grimaldi A, Terova G, Saroglia M, de Eguileor M (2005) Oxygen availability causes morphological changes and a different VEGF/FIkât? 1/HIFât? 2 expression pattern in sea bass gills. Italian Journal of Zoology 72: 103-111

Rutjes HA, Nieveen MC, Weber RE, Witte F, Van den Thillart GE (2007) Multiple strategies of Lake Victoria cichlids to cope with lifelong hypoxia include hemoglobin switching. Am J Physiol Regul Integr Comp Physiol 293: R1376-1383

Saha D, Patgaonkar M, Shroff A, Ayyar K, Bashir T, Reddy KV (2014) Hemoglobin expression in nonerythroid cells: novel or ubiquitous? Int J Inflam 2014: 803237

Tamura K, Stecher G, Peterson D, Filipski A, Kumar S (2013) MEGA6: Molecular Evolutionary Genetics Analysis version 6.0. Mol Biol Evol 30: 2725-2729

Terova G, Cattaneo AG, Preziosa E, Bernardini G, Saroglia M (2011) Impact of acute stress on antimicrobial polypeptides mRNA copy number in several tissues of marine sea bass (Dicentrarchus labrax). BMC Immunol 12: 69

Tezel G, Yang X, Luo C, Cai J, Kain AD, Powell DW, Kuehn MH, Pierce WM (2010) Hemoglobin expression and regulation in glaucoma: insights into retinal ganglion cell oxygenation. Invest Ophthalmol Vis Sci 51: 907-919

Tiedke J, Gerlach F, Mitz SA, Hankeln T, Burmester T (2011) Ontogeny of globin expression in zebrafish (Danio rerio). J Comp Physiol B 181: 1011-1021

Tine M, Kuhl H, Gagnaire PA, Louro B, Desmarais E, Martins RS, Hecht J, Knaust F, Belkhir K, Klages S, Dieterich R, Stueber K, Piferrer F, Guinand B, Bierne N, Volckaert FA, Bargelloni L, Power DM, Bonhomme F, Canario AV, Reinhardt $R$ (2014) European sea bass genome and its variation provide insights into adaptation to euryhalinity and speciation. Nat Commun 5: 5770

Tortonese E (1986) Moronidae. In: Whitehead PJP, Bauchot ML, Hureau JC, Nielsen J, E. T (eds) Fishes of the North-eastern Atlantic and the Mediterranean, Paris, pp 793-796

Ullal AJ, Wayne Litaker R, Noga EJ (2008) Antimicrobial peptides derived from hemoglobin are expressed in epithelium of channel catfish (Ictalurus punctatus, Rafinesque). Developmental \& Comparative Immunology 32: 1301-1312

Verde C, Balestrieri M, de Pascale D, Pagnozzi D, Lecointre G, di Prisco G (2006) The oxygen transport system in three species of the boreal fish family Gadidae. Molecular phylogeny of hemoglobin. J Biol Chem 281: 22073-22084

Verde C, Carratore V, Riccio A, Tamburrini M, Parisi E, Di Prisco G (2002) The functionally distinct hemoglobins of the Arctic spotted wolffish Anarhichas minor. J Biol Chem 277: 36312-36320

Verde C, Vergara A, Mazzarella L, di Prisco G (2008) The hemoglobins of fishes living at polar latitudes - current knowledge on structural adaptations in a changing environment. Curr Protein Pept Sci 9: 578-590

von der Heyden S, Toms JA, Teske PR, Lamberth SJ, Holleman W (2015) Contrasting signals of genetic diversity and historical demography between two recently diverged marine and estuarine fish species. Marine Ecology Progress Series 526: 157-167 
Weber RE (1990) Functional significance and structural basis of multiple hemoglobins with special reference to ectothermic vertebrates.: In: Animal Nutrition and Transport Processes. 2. Transport, Respiration and Excretion: Comparative and Environmental Aspects. Comparative Physiology, Basel:Karger, pp 58-75

Weber RE (2000) Adaptations for Oxygen Transport: Lessons from Fish Hemoglobins Hemoglobin Function in Vertebrates: Molecular Adaptation in Extreme and Temperate Environments. Springer Milan, Milano, pp 23-37

Wetten OF, Nederbragt AJ, Wilson RC, Jakobsen KS, Edvardsen RB, Andersen O (2010) Genomic organization and gene expression of the multiple globins in Atlantic cod: conservation of globin-flanking genes in chordates infers the origin of the vertebrate globin clusters. BMC Evol Biol 10: 315

Zambonino-Infante JL, Cahu CL, Peres A, Quazuguel P, Le Gall MM (1996) Sea bass (Dicentrarchus labrax) larvae fed different Artemia rations: growth, pancreas enzymatic response and development of digestive functions. Aquaculture 139: 129-138

Zhou J, Qiao X, Xiao L, Sun W, Wang L, Li H, Wu Y, Ding X, Hu X, Zhou C, Zhang J (2010) Identification and characterization of the novel protein CCDC106 that interacts with $p 53$ and promotes its degradation. FEBS Lett 584: 1085-1090 
Table 1: Specific primers used for real-time amplification of $\mathrm{Hb}$ and reference (18S and EF1 $\alpha$ ) genes. Genbank Accession numbers are also given.

\begin{tabular}{|c|c|c|c|}
\hline Gene names & $\begin{array}{l}\text { Accession } \\
\text { numbers }\end{array}$ & Forward primer sequences ( $\left.5^{\prime}-3^{\prime}\right)$ & Reverse primer sequences $\left(5^{\prime}-3^{\prime}\right)$ \\
\hline LA-Hb $\alpha 1$ & KX196178 & TTTCCCATGAGAGAGCAGGT & TCAGATGCGCTTCTTAGGATGT \\
\hline LA-Hb $\alpha 2$ & KX196180 & CAGTGGGACAGGATCTTGAAGT & GGTGATGGGTGGAATCAATC \\
\hline $\mathrm{LA}-H b \beta 1$ & KX196179 & CCCGACAACTTCAAACTGCT & CCTGCGTCTCTGGTGTGAAG \\
\hline $\mathrm{MN}-\mathrm{Hb} \alpha \mathbf{1}$ & KX196190 & GGCCAGGATGCTGACTGTA & CCAGCAAGGTCATCCATCTT \\
\hline $\mathrm{MN}-\mathrm{Hb} \alpha 2$ & KX196188 & CCTGCCAACTTCAAGATTCTG & TTTCTCAGACAAGGCACGAG \\
\hline $\mathrm{MN}-H b \alpha 3$ & KX196184 & ACAGACAAGATGACCAGTCTCACT & GCCAATGTCCTCTGCCTTC \\
\hline $\mathrm{MN}-\mathrm{Hb} \alpha 4$ & KX196183 & ACAGACAAGATGACCAGTCTCACT & GCCAATGTCCTCTGCCTTT \\
\hline $\mathrm{MN}-\mathrm{Hb} \alpha 5$ & KX196181 & ACAGACAAGATGACCAGTCTCACA & GCCAATGTCCTCTGCCTTC \\
\hline $\mathrm{MN}-H b \beta 1$ & KX196191 & TGATTTGAGCAAAGATCCTGAA & CATGGACGACATCAAGAACG \\
\hline $\mathrm{MN}-H b \beta 2$ & KX196189 & GTCAGCCAGCAGCCTGAAAT & GCAGCTCTTTCCAGGTGTCT \\
\hline $\mathrm{MN}-H b \beta 3$ & KX196187 & CAGAAGCTTTGGCAAGAGTG & GCTGCTACTTTGGCGTTACC \\
\hline $\mathrm{MN}-\mathrm{Hb} \beta 4$ & KX196186 & GTCGTTTACCCCTGGACTCA & GTTTTGCGACCATCGGATTT \\
\hline $\mathrm{MN}-H b \beta 5$ & KX196185 & ACCATCCAGGACATCTTCTCT & GTTTTGCGACCAACGGATTC \\
\hline $\mathrm{MN}-\mathrm{Hb} \beta 6$ & KX196182 & ACCATCCAGGACATTTTCTCC & GTTTTGCGACCAACGGATTC \\
\hline $18 S$ & AY831388.1 & TGGTTCCTTTGATCGCTCTT & AGCAGCTCGTTGGCATGTAT \\
\hline Ef1 $\alpha$ & AJ866727.1 & GCTTCGAGGAAATCACCAAG & СААССTTCСАTСССTTGAАC \\
\hline
\end{tabular}


Table 2: Lengths in base pairs (bp) of exons, introns, predicted coding (CDS) and amino acid (AA) sequences for the European sea bass $\mathrm{Hb \alpha}$ and $\mathrm{Hb} \beta$ genes.

\begin{tabular}{|c|c|c|c|c|c|c|c|c|}
\hline \multirow[b]{2}{*}{ Cluster } & \multicolumn{8}{|c|}{ Length (bp) } \\
\hline & Genes & exon 1 & intron 1 & exon 2 & intron 2 & exon 3 & CDS & AA \\
\hline \multirow[t]{11}{*}{ MN } & $H b \alpha 1$ & 95 & 107 & 208 & 176 & 129 & 432 & 143 \\
\hline & $H b \alpha 2$ & 95 & 105 & 208 & 506 & 129 & 432 & 143 \\
\hline & $H b \alpha 3$ & 98 & 422 & 208 & 180 & 129 & 435 & 144 \\
\hline & $H b \alpha 4$ & 98 & 110 & 208 & 184 & 129 & 435 & 144 \\
\hline & $H b \alpha 5$ & 98 & 348 & 208 & 180 & 129 & 435 & 144 \\
\hline & $H b \beta 1$ & 92 & 142 & 223 & 94 & 129 & 444 & 147 \\
\hline & $H b \beta 2$ & 92 & 110 & 223 & 97 & 129 & 444 & 147 \\
\hline & $H b \beta 3$ & 92 & 737 & 223 & 106 & 129 & 444 & 147 \\
\hline & $H b \beta 4$ & 92 & 143 & 223 & 99 & 129 & 444 & 147 \\
\hline & $H b \beta 5$ & 92 & 146 & 223 & 99 & 129 & 444 & 147 \\
\hline & $H b \beta 6$ & 92 & 145 & 223 & 99 & 129 & 444 & 147 \\
\hline LA & $H b \alpha 1$ & 95 & 629 & 208 & 355 & 129 & 432 & 143 \\
\hline & $H b \alpha 2$ & 92 & 1103 & 205 & 1010 & 129 & 426 & 141 \\
\hline & $H b \beta 1$ & 92 & 91 & 223 & 246 & 129 & 444 & 147 \\
\hline
\end{tabular}


Table 3. Relative expression of $\mathrm{Hb} \alpha$ and $\mathrm{Hb} \beta$ genes at different developmental stages of European sea bass as normalized with expression levels of $18 \mathrm{~S}$ rRNA. MN-Hbß3 values are not shown since Cq values were up to 35 from $12 \mathrm{hpf}$ until juvenile stage. Different letters show significant difference for the expression of one gene among tissues (One-way ANOVA, p<0.05). ND means Not Detected.hpf: hours post fertilization; dph: days post hatching.

\begin{tabular}{|c|c|c|c|c|c|c|c|c|c|c|}
\hline Gene & $12 \mathrm{hpf}$ & $60 \mathrm{hpf}$ & $5 \mathrm{dph}$ & $13 \mathrm{dph}$ & $19 \mathrm{dph}$ & $26 \mathrm{dph}$ & $33 \mathrm{dph}$ & $45 \mathrm{dph}$ & Juvenile & pvalue \\
\hline LA- $H B \alpha 1$ & $1^{*} 10^{-5} / \mathrm{a}$ & $1 * 10^{-5} / \mathrm{a}$ & $5^{*} 10^{-5} / \mathrm{a}$ & $9^{*} 10^{-5} / \mathrm{a}$ & $7^{*} 10^{-5} / \mathrm{a}$ & $3^{*} 10^{-5} / \mathrm{a}$ & $1^{*} 10^{-3} / \mathrm{a}$ & $0,07 / a$ & $1,1 / b$ & $1^{*} 10^{-6}$ \\
\hline $\mathrm{LA}-H B \alpha 2$ & $2 * 10^{-3} / \mathrm{a}$ & $1 * 10^{-3} / \mathrm{a}$ & $0,18 / \mathrm{b}$ & $0,2 / b$ & $0,13 / b$ & $0,11 / \mathrm{b}$ & $0,43 / b$ & $0,91 / \mathrm{c}$ & $1,26 / \mathrm{c}$ & $8 * 10^{-6}$ \\
\hline $\mathrm{MN}-H B \alpha 1$ & $0,01 / a$ & $6^{*} 10^{-3} / \mathrm{a}$ & $5^{*} 10^{-3} / \mathrm{a}$ & $0,05 / a$ & $0,01 / a$ & $0,01 / \mathrm{a}$ & $0,01 / a$ & $0,03 / a$ & $1,43 / b$ & $1^{*} 10^{-5}$ \\
\hline $\mathrm{MN}-H B \alpha 2$ & ND & ND & $8 * 10^{-5} / \mathrm{a}$ & $6 * 10^{-5} / \mathrm{a}$ & $3 * 10^{-5} / \mathrm{a}$ & $2^{*} 10^{-4} / \mathrm{a}$ & $0,01 / a$ & $0,41 / \mathrm{b}$ & $1 / \mathrm{c}$ & $1 * 10^{-6}$ \\
\hline $\mathrm{MN}-H B \alpha 3$ & $2 * 10^{-4} / a$ & $9^{*} 10^{-5} / \mathrm{a}$ & $0,75 / c$ & $0,21 / a b$ & $0,48 / \mathrm{bc}$ & $0,25 / a b$ & $0,47 / \mathrm{bc}$ & $0,19 / a b$ & $0,01 / a$ & $8 * 10^{-4}$ \\
\hline $\mathrm{MN}-\mathrm{HB} \alpha 4$ & $1 * 10^{-5} / \mathrm{a}$ & $1 * 10^{-5} / \mathrm{a}$ & $0,03 / a$ & $6^{*} 10^{-3} / \mathrm{a}$ & $0,05 / a$ & $0,07 / a$ & $0,25 / b$ & $0,85 / \mathrm{c}$ & $0,01 / \mathrm{a}$ & $1 * 10^{-6}$ \\
\hline$M N-H B \alpha 5$ & $4^{*} 10^{-5} / \mathrm{a}$ & $4^{*} 10^{-5} / \mathrm{a}$ & $0,13 / \mathrm{bc}$ & $0,07 / \mathrm{c}$ & $0,07 / \mathrm{c}$ & $0,12 / \mathrm{bc}$ & $0,35 / \mathrm{bd}$ & $0,67 / d$ & $2^{*} 10^{-3} / a$ & $6^{*} 10^{-5}$ \\
\hline $\mathrm{LA}-H b \beta 1$ & $1 * 10^{-5} / \mathrm{a}$ & ND & $3^{*} 10^{-3} / \mathrm{a}$ & $7 * 10^{-3} / a$ & $0,01 / a$ & $0,03 / a$ & $0,22 / b$ & $0,75 / \mathrm{c}$ & $1,04 / d$ & $1 * 10^{-6}$ \\
\hline $\mathrm{MN}-\mathrm{Hb} \beta 1$ & $0,06 / a$ & $0,01 / a$ & $0,33 / a b$ & $0,17 / / a$ & $0,10 / a$ & $0,18 / \mathrm{a}$ & $0,32 / a b$ & $0,32 / a b$ & $1 / b$ & $1^{*} 10^{-3}$ \\
\hline $\mathrm{MN}-\mathrm{Hb} \beta 2$ & ND & ND & $4^{*} 10^{-5} / \mathrm{a}$ & $5^{*} 10^{-5} / a$ & $4^{*} 10^{-5} / \mathrm{a}$ & $7^{*} 10^{-5} / \mathrm{a}$ & $3^{*} 10^{-3} / \mathrm{a}$ & $0,12 / \mathrm{b}$ & $1 / \mathrm{c}$ & $1^{*} 10^{-6}$ \\
\hline $\mathrm{MN}-\mathrm{Hb} \beta 4$ & $7^{*} 10^{-5} / \mathrm{a}$ & $1^{*} 10^{-4} / \mathrm{a}$ & $0,55 / \mathrm{b}$ & $0,45 / \mathrm{b}$ & $0,56 / \mathrm{b}$ & $0,36 / \mathrm{b}$ & $0,63 / \mathrm{b}$ & $1 / c$ & $0,54 / \mathrm{b}$ & $7^{*} 10^{-6}$ \\
\hline $\mathrm{MN}-\mathrm{Hb} \beta 5$ & $4^{*} 10^{-4} / \mathrm{a}$ & $1 * 10^{-4} / \mathrm{a}$ & $0,87 / \mathrm{c}$ & $0,4 / b c$ & $0,72 / \mathrm{c}$ & $0,4 / \mathrm{bc}$ & $0,36 / \mathrm{bc}$ & $0,06 / \mathrm{b}$ & $6^{*} 10^{-3} / \mathrm{b}$ & $5^{*} 10^{-3}$ \\
\hline $\mathrm{MN}-\mathrm{Hb} \beta 6$ & ND & $1 * 10^{-5} / \mathrm{a}$ & $8 * 10^{-3} / \mathrm{b}$ & $0,01 / \mathrm{b}$ & $0,07 / \mathrm{bc}$ & $0,08 / b c$ & $0,29 / c$ & $0,68 / d$ & $0,01 / \mathrm{b}$ & $1 * 10^{-4}$ \\
\hline
\end{tabular}




\section{MN cluster}

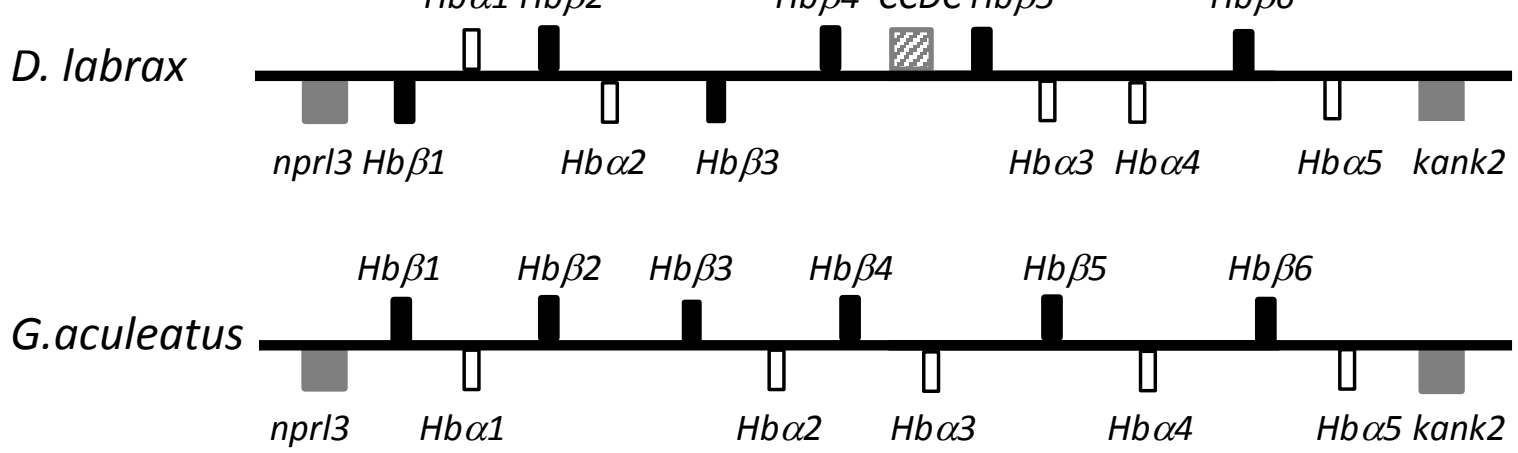

\section{LA cluster}

D. labrax

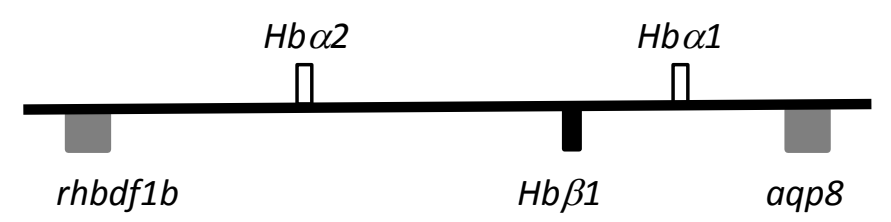

\section{$\alpha$-globin \\ $\beta$-globin}

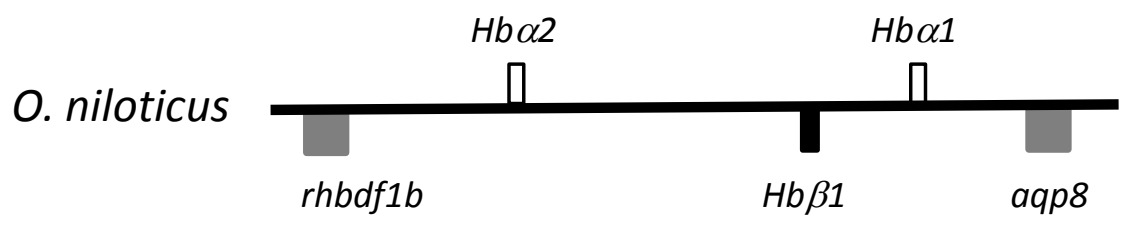

Fig1. Genomic organization of the European sea bass MN- and LA-clusters.

Genomic structures of the D. labrax $M N$ - and LA-clusters are compared with G. aculeatus and O. niloticus, respectively. The genes in the forward and the reverse orientations are shown boxed above and below the line, respectively. $H b \alpha$ and $H b \beta$ genes are represented by white and black boxes, respectively. Flanking genes are shown in grey and the inserted gene is shaded. 


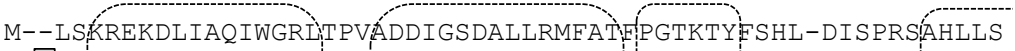
M-SLN:AKDKSVVKA IWAKVIS PKSAEIGAEALARMLTVIPQTKTY FSHWSDLS PES:PQVRA

LA-Hb $\alpha 1$

$\mathrm{MN}-\mathrm{Hb} \alpha 2$

$\mathrm{MN}-\mathrm{Hb} \alpha 3$

$\mathrm{MN}-\mathrm{Hb} \alpha 5$

$\mathrm{MN}-\mathrm{Hb} \alpha 4$

LA- $H b \alpha 2$

$\mathrm{MN}-\mathrm{Hb} \alpha 1$

LA-Hb $\alpha 1$

$\mathrm{MN}-\mathrm{Hb} \alpha 2$

$\mathrm{MN}-\mathrm{Hb} \alpha 3$

MN-Hb $\alpha 5$

$\mathrm{MN}-\mathrm{Hb} \alpha 4$ M-SLTQKDKAAVKALWAKISKSADAIGSDALSRMLTVYPQTKTY SHWPDMSPGSAPVKA M-SLTAKDKAAVKAFWGKVISGKAEDIGTDALSRMLVVIPQTKTY SHWKDLSPGSAPVRK MTSLTAKDKDAVRTFWAKV:SGKAEDIGADALARMLAVYPQTKTYFAHWKDQSPTSPSARK MTSLTAKDKDAVRTFWAKVSGKAEDIGADALARMLAVIPQTKTYFAHWKDQSPTSPSARK MTSLTA_KDKDAVRTFWAKDSGKA_EDIGAEALARMLAVYPQTKTYFSHWKDQSPTSP्SARK

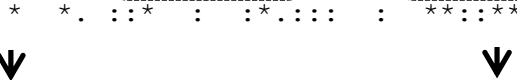

HGKKIVLAIAEGAQDi SQĹfAVNAPLQTLHAỲLLRIDPTNÉKLFSHCMLVTLACHMGDDF HGATIMAAVGDAVGKMDD:LAGGLISLSELHAFKLRVDPANFRI LAHNI I LVLAMLFPADF HGKKVMGGINLAVSK:IDDITNGLLELSEQHAFOLRVDPTNEKILSHCVLVVVSSMFPNDF HGKTVMSGVADAVSKIDD:LNAGLLNLSELHAFTLRVDPANFKILAHNILVVLS IMRPEDF HGITVMTGVADAVTK:IDDEKGGLLSLSELHAFTLRVDPANEKILSHNILVVMAIMFPTDF HGITVMTGVADAVTKIDD:LKGGLLSLSELHAFTLRVDPANFKILSHNILVVMAIMFPTDF HGITVMTGVADAVS, IDDEKGGLLSLSELHAETLRVDPANE_KILSHNI LVVMAIMFPTDF

LA- $\mathrm{Hb} \alpha 2$

$\mathrm{MN}-\mathrm{Hb} \alpha \mathbf{1}$

LA-Hbo1

$\mathrm{MN}-\mathrm{Hb} \alpha 2$

$\mathrm{MN}-\mathrm{Hb} \alpha 3$

$\mathrm{MN}-\mathrm{Hb} \alpha 5$
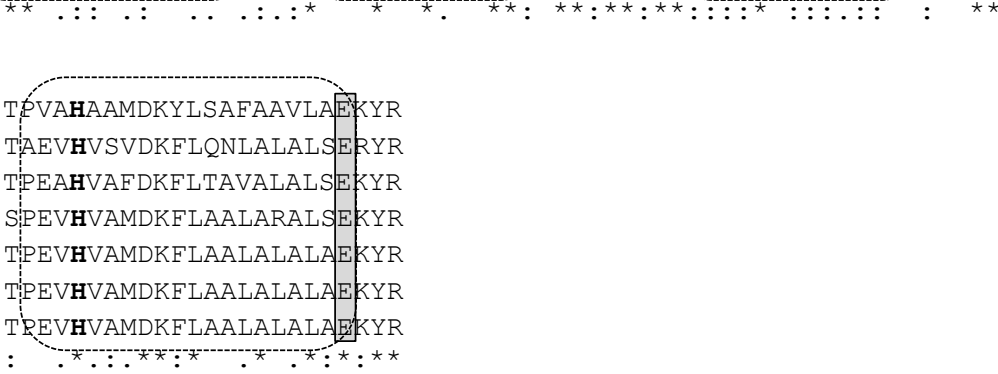

Fig2. Amino acid sequence alignment of European sea bass $\mathrm{Hb} \alpha$.

The amino acid similarities between all proteins are indicated as follows: asterisks indicate identical amino acids, colons or dots indicate similar amino acids and empty spaces represent absence or a low degree of similarity. Predicted alpha-helices are shown in dashed boxes. Histidine residues included in oxygen binding are indicated by arrows. Amino acids potentially involved in the Root effect are boxed in grey. The residues responsible for the Bohr effect are indicated in bold. 
$\mathrm{MN}-\mathrm{Hb} \beta 3$

$\mathrm{MN}-\mathrm{Hb} \beta 1$

LA- $H b \beta 1$

$\mathrm{MN}-\mathrm{Hb} \beta 2$

$\mathrm{MN}-\mathrm{Hb} \beta 6$

$\mathrm{MN}-\mathrm{Hb} \beta 4$

$\mathrm{MN}-\mathrm{Hb} \beta 5$

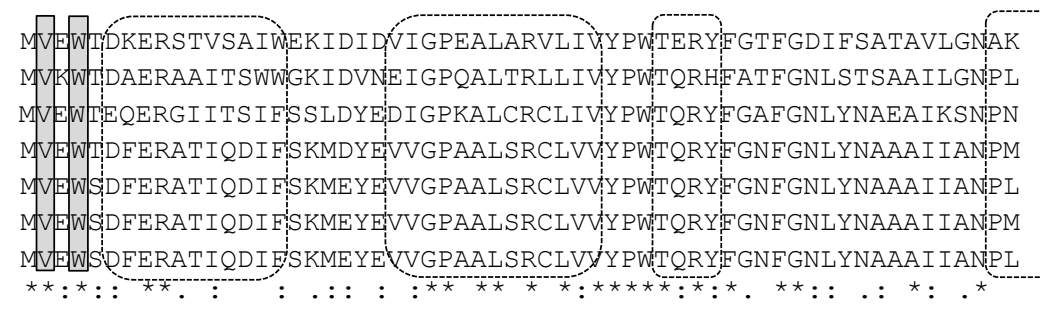

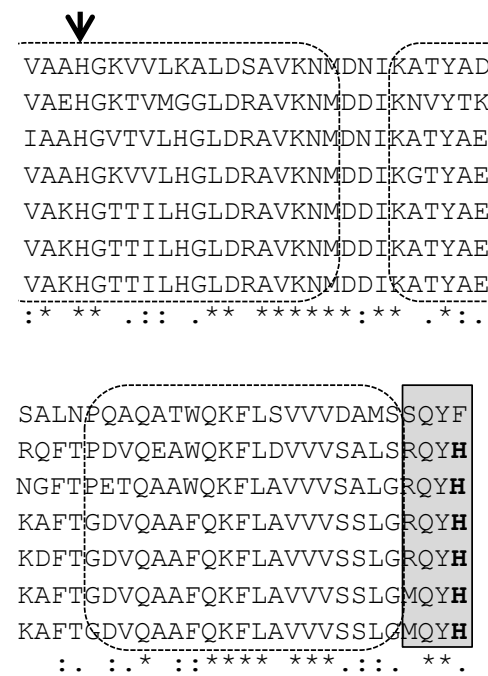

$\mathrm{MN}-\mathrm{Hb} \beta 3$

$\mathrm{MN}-\mathrm{Hb} \beta 1$

LA- $H b \beta 1$

$\mathrm{MN}-\mathrm{Hb} \beta 2$

$\mathrm{MN}-\mathrm{Hb} \beta 6$

$\mathrm{MN}-\mathrm{Hb} \beta 4$

$\mathrm{MN}-\mathrm{Hb} \beta 5$

\section{Fig3. Amino acid sequence alignment of European sea bass $H b \beta$.}

The amino acid similarities between all proteins are given as follows: asterisks indicate identical amino acids, colons or dots indicate similar amino acids and empty spaces represent absence or low degree of similarity. Predicted alpha-helices are shown in dashed boxes. Histidine residues included in oxygen binding are indicated by arrows. Amino acids potentially involved in the Root effect are boxed in grey. The C-terminal His residue responsible for the Bohr effect is indicated in bold. 
Fig4. Bayesian phylogenetic tree of $\mathrm{Hb} \alpha$ sequences

Phylogenetic analysis was performed on both $\mathrm{Hb} \alpha$ and $\mathrm{Hb} \beta$ following the method detailed in "Materials and Methods" section but only Hba sequences are presented here. European sea bass $\mathrm{Hb} \alpha$ genes are marked with asterisks. Hba amino acid sequences from other species were issued from the translation of nucleotide sequences listed in a previous work by Opazo and collaborators (10). Monophyletic clades are represented by a triangle. Clades containing $\mathrm{Hb} \beta$ genes of European sea bass and other species have been collapsed in an $\mathrm{Hb} \beta$ clade. Branches are color coded according to the location of the genes: $M N$-linked Hbs are shown in blue and LA-linked Hbs are shown in red.

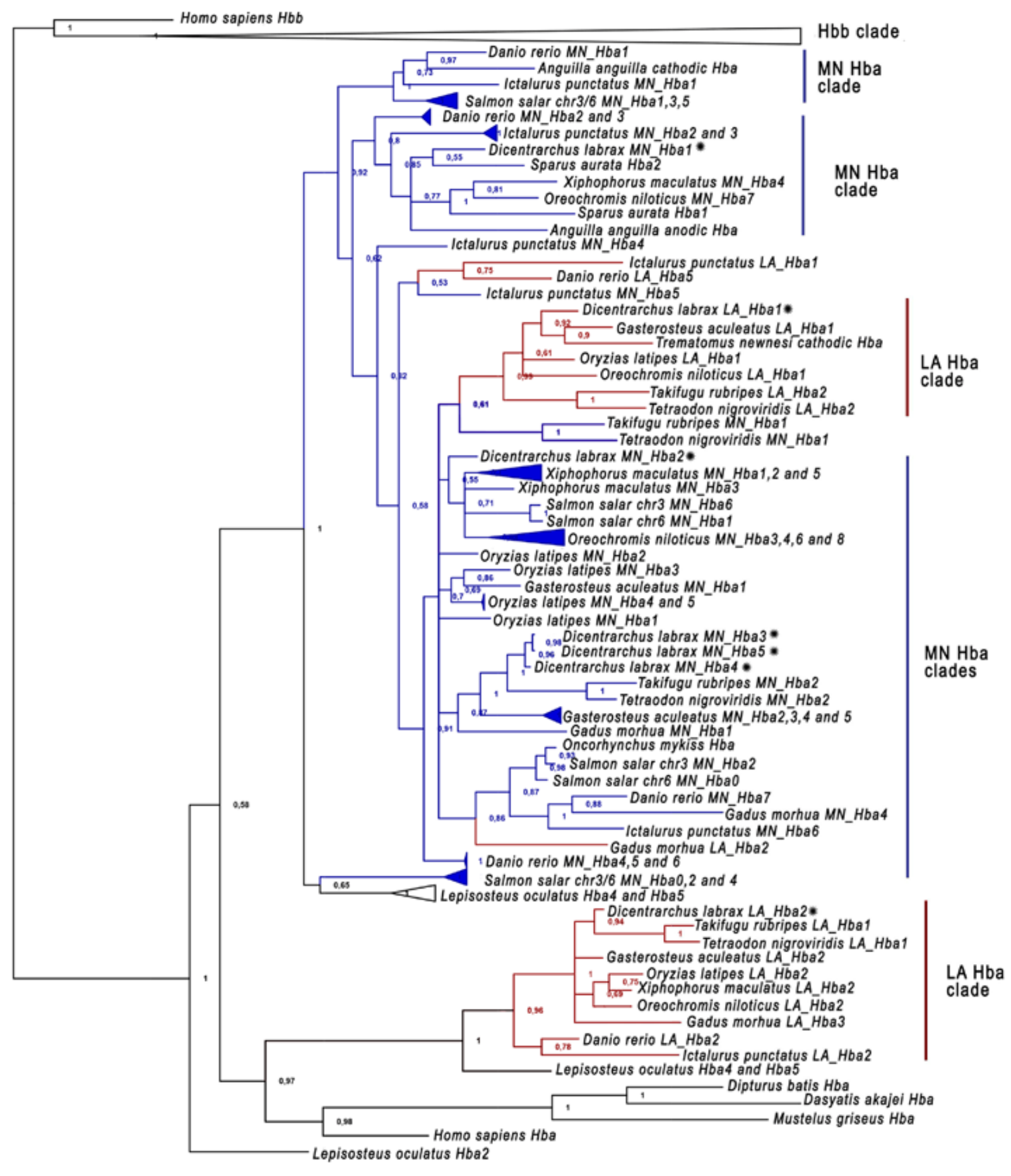




\section{Fig5. Bayesian phylogenetic tree of $\mathrm{Hb} \beta$ sequences}

Phylogenetic analysis was performed on both $\mathrm{Hb} \alpha$ and $\mathrm{Hb} \beta$ following the method detailed in "Materials and Methods" section but only $\mathrm{Hb} \beta$ sequences are presented here. European sea bass $\mathrm{Hb} \beta$ genes are marked with asterisks. $\mathrm{Hb} \beta$ amino acid sequences from other species were issued from the translation of nucleotide sequences listed in a previous work by Opazo and collaborators (10). Monophyletic clades are represented by a triangle. Clades containing $\mathrm{Hb} \alpha$ genes of European sea bass and other species have been collapsed in an $\mathrm{Hb} \alpha$ clade. Branches are color coded according to the location of the genes: $M N$-linked Hbs are shown in blue and LA-linked Hbs are shown in red.

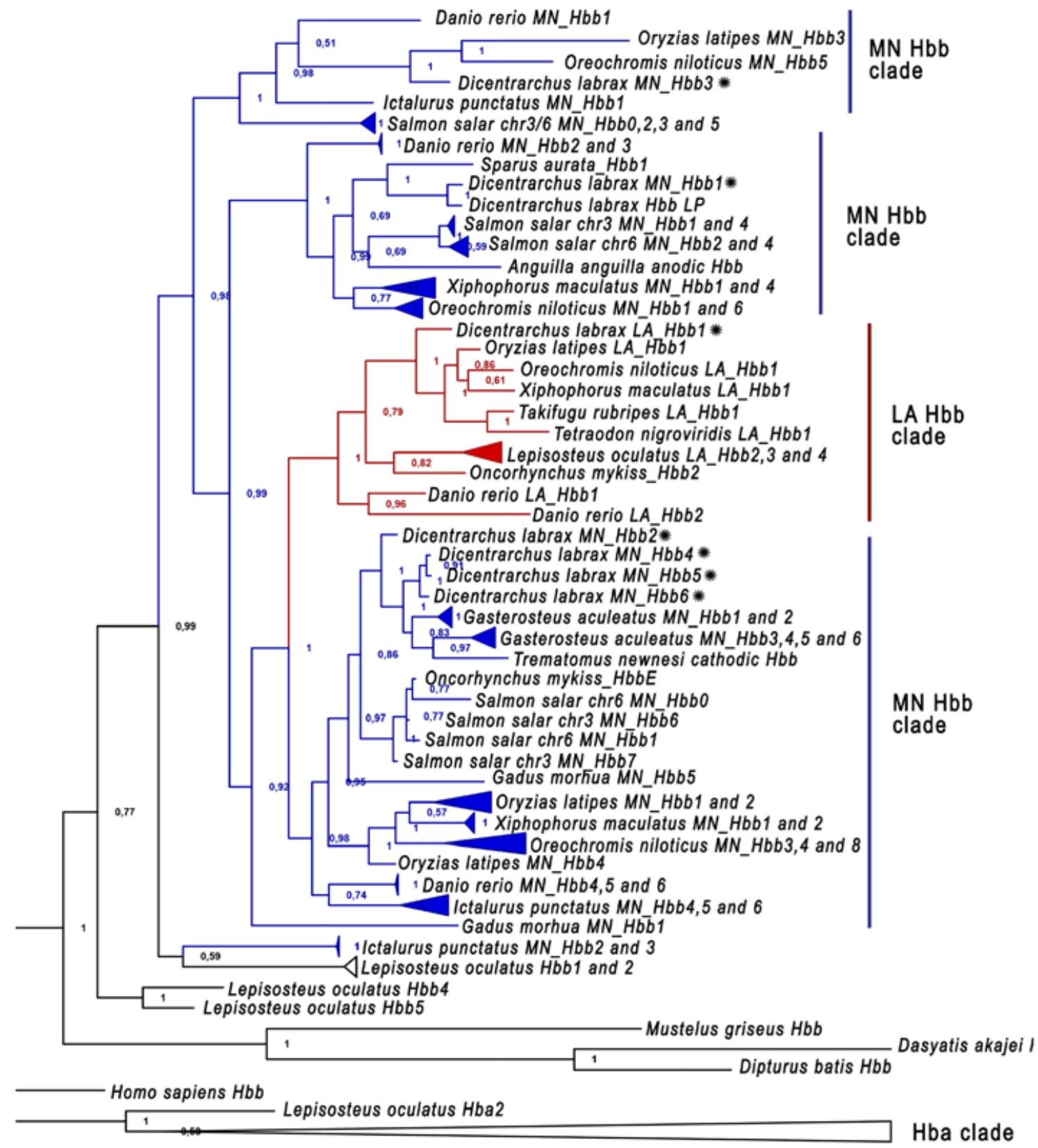




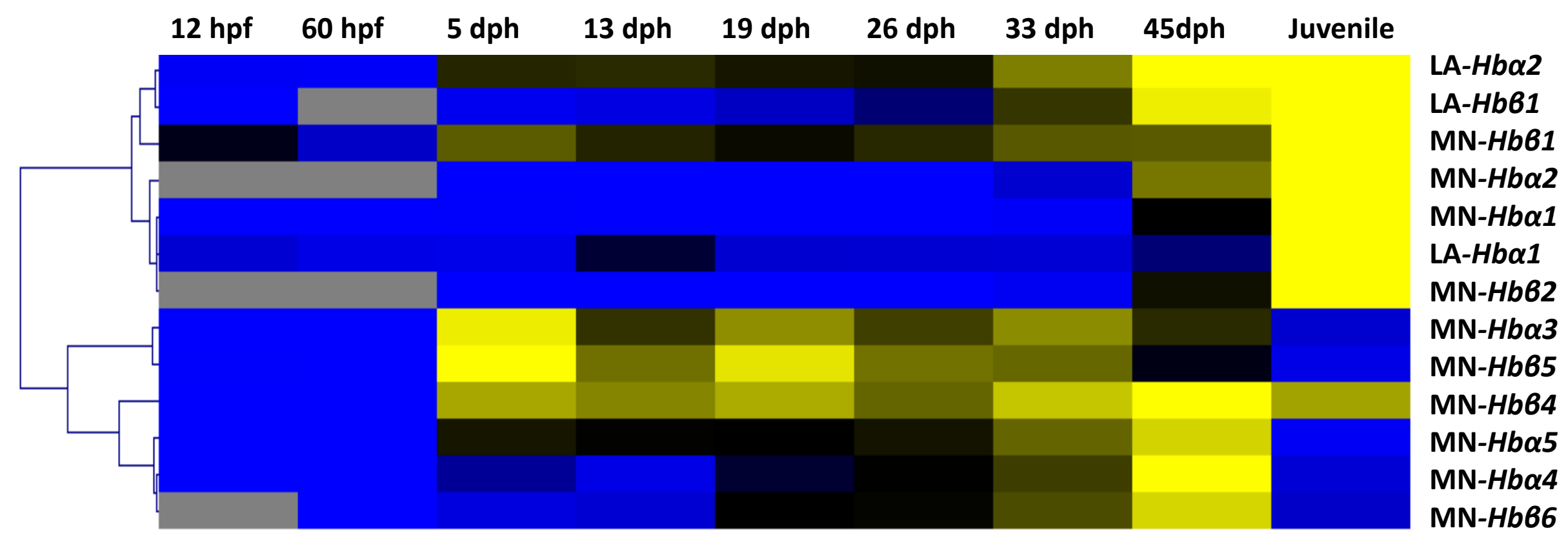

Fig6. Relative expression profiles of $\mathrm{Hb} \alpha$ and $\beta$ genes during development of European sea bass. The relative expression profiles of $\mathrm{Hb} \alpha$ and $\beta$ genes during development of European sea bass were determined using qPCR. $\mathrm{MN}-\mathrm{Hb} \beta 3$ expression profile is not shown since Cq values were up to 35 from $12 \mathrm{hpf}$ until juvenile stage. Following normalization with $18 \mathrm{~S}$ rRNA levels, relative expression of each gene throughout the life stages (shown in table 3) were subjected to hierarchical clustering and a heat map was generated. Relative expression level of each gene is depicted by a colour scale: blue and yellow indicating respective low and high relative expression levels among developmental stages. Hpf: hours post fertilization; dph: days post hatching. 
$>$ LA_Cluster

TTGĀTATGAATGATCGCACTTTCTGTTATCTGTTTACAATCTTTTTGTACTCATTGTTTGGGTCAAAGTCCTA AATAgAAAACCGTTCTTTAATAgCTTTATTACTGAAAAGACGGTACAAGGTCAGGTCACTACATAAGATAATA AAgCTAGATGAGAATCATAAACTGGTGCTCCTAGATTAACTGAAACAGCAATAAGTTACAATAAAAAGCTTTC TAAATACAGATGTATCTCATATATTCATCTTAAAATAATGGGTGAAGATGGATCATCTACCTTGCCAACCTAT CTGTTCAAGAGACTGTAAGACTGTTATGTGAAATTTGAATATATGGAATTAAAGTTTGAATATGGAATGAAAT CCTATGTGATCAGGTCACAATACTTTCTATACCTAAAGCATGTCATAATATGTGTAAGATATTCCTTTATTTG GTGTCACTGGTGGAAGTATTCAGATCATTTACATAAAGGTATACTCAAGTGAAAGCACAGATGTGTTATCAGC TAGAATGTTCAAAAGTAAAAGTATTGATTATGCATTACTCGTTGGATGGTGCTGTATGACACATACATATTAT ATCATAGTTCTGTGTGCACTATTTAAAGTAGCCTTTTGCTGTTTGAGGTGGAGCTGTTGGTAGTTTAAAACAT TTCTGTCTTCAATtGgGCCCACAGTTTTTCCTTTAAACAAAAGGAAAAAACAAAATCAGTGCAGTAAAATA TTTCTAACTTTATTTGGAAAGTTGTCCAGGTTATTTGCATTGAAAATATAGATATGCTGAGACTATAATGAC CCAATTATATACTATGATGCCTTGATAAGAAGGAATAAAGTAGCATTAAACAGAAAAGAAAAAATAAGTACAT CAAAATTGTACTCACTGTCGGCTTGGTGTCTTGATGAAGAGCGTTGTGTAACAGTTCAGTCCAATTCTCCCTT TAGTGTACAATTAGGTTGATATCTGCTGACTGTCAAGGCCATAGCGTATAATTCATGTCATTTTATACAAATT AAAGTATTCGGTAACCCCTCCTGCTCTGAAGGTCAGCATCTGAATTGTTTTTTCTTATAGTTTTACATTTACT TTAGTTAAGTAAAAATACCAATACAACCATGTAAAAATACTCCATTACTTGTAAAAGTCCTGCATTTGTTCTT GATCATTAAAATGTACTGATTCTGCAGAATATATGACCGTATTATTTTTAATACTGATGCAGTAATACAAAAG TACTGTGGTAACTTCTAATCACTTAGGTAGTTGAGTGTAGTAGTTCCCAACCTAGGGGTCATAAATCTCAACA АтСАTTTGACCTATCTGGACCTTAAAAGgTTTAGATGGGAAATGTCTCTATACTGGAACTGCTCACAAATCAT TAACATCTGAAATGTAACACAATGAGATACTGTGAGGGGTCACAAGCCAAAAAGGTTCGGAAACACGGGTTTA AACTCTAACAGTGTGTCGCATGGGGCGTCCTGACCACTGAGGACTTAACCTTGATGCATGGTGTAAAGTCTGT ATTCCAGGTCTATCTTATCTATGTAGGTCACCTATAGCAAGCATGTGCATACGAGTACAGAAATTATGATGAT TAAATTGCACATGATTGATCCCTAACTGATCCCTCTGACTGACATCAACCTTGAACAGCTCGTGCCTTGTTTT GAATCTTATCATTCTTTTATGAAAGGTAACTTGAGGACTCCTTAAAGGACAGGATTACCTGGCTTGACTCCAT CAGACAGAGCTGGAAAGGCGTCAAAGAACCTGTTTGAGGCGTGGGTTGTTTTGATGTTATGGATTTTCACCTT CAGAACATGCTTCACCTTTGATGTTTTTATGACCTGTGATGCAATTCAGCAACAGTGTGCAGTCTGCTGTTGA TGATTATAAATACCCAGAAAACCTTGCAGTGCCACAGTCCGAAAGAGGTGCAGAATGTCTTTGAGAATCATCC TGGAACAAAAGTCTCTTACAGGCAACAATGGGACTTGAGAAAATGGAAATGGAAGACACAGACTCTGCTCTGA TGGAGAAGGGCAAGAAGCCGCCTGTGTCCAAACCTCCCAACAAATATGAGAAGCTGTTCCAGCCCTGCCTGGC CGAGATAGTGGGGACCATGTTCTTCGTTTTCATCGGCTGTGTGTCTGTCATTGAGAATGTGCCAGCGGCTGGA CGGCTGCAGCCAGCCTTGGTGCACGGGCTGGCTGTGGCAGTGATGGTGGCGGTTATGGATAACATCAGGTATT TTGCGTAAAGAATAGAATGACATAAAGTCAGATGCTGTGTATTTTAATTTTTGCTTGCTTGCTTTATGTTTTT GTGTGCAACATCTTTGAATAATGATCAACCTCAGCCATTTGGTATATTTTGATATTTTGAGGGGAATGGGTCT CAGTGTCCCACCTGATCTCAACTTTTCTCTGCTGTCTCTTCCTCTAGTGGCTCCCATTTCAACCCTGCCTTCA СTATTGCCATCTACCTGTGTGGGGGCATGGAGCTGGCCATGGTAGGACCCTACCTTGCCAGCCAGCTGATTGG AGGAGTTCTGGGAGCTGGAATGGCCATGGTCAGGTGAAAGTTCACTGCACAGTATTCTGATTTTTTCACACAA AтTTTGAATATTTATAATTATAATATATAATCATAATTCAAAAGTGACCATAAAATCTTTAATAATACAA TTTTTAGATAATGACCCCTGCAGGTCGTTACAACAACGCCACGGGAGCAGCGTTTGATATCCTCAAGTCAGAC AGTCAGCTGTCTGGAGCCATCTTTGGGGAGGTGGCCATGACCTGCTTGGTCACCATGGTGGTGCTGCTGGTGG CGGTCAACGGCAAGACAAAAAACCCGCTGGCTCCATTCCTAGTTGGCTGTACCGTCATCATTAACATCCTGGC AGGGTGAGATAAAGGGGGCGATGACCACAGTGTTTATTGTGTCCGCACTGTGTTAAACATTTACACCCAACTG GACCTGAACGTTGCAGTCAGTTGCTATATTCACGAATTGAGGATTCGCATATCTTGCTTCACAACCAACATTT AАTTCAAAGTAAAAAAAAAAAAAAGTTATAAAATAATGTTTCCCAAAGTGAAATAGTTAAAAAAAAAAAAATT TAAAAACAGCAGCCAACAACACAAAGGACATTAAAGGCGTGGTGGTAGAGATATCTCTCTAAAGATGTGTTTT TTAАTTTATGTTTTTTTATTTGAAATGCACTATTACTTTCATTAAATTAАTTTTGCATTTTTTTTTTTTTTTT GCAAATGTCTTACTTTATTCGATTGTTTCGAAATAAAATGCTTTTAAGTTAATGACTTAAAAATCAATTAACC AATACTTGAGGGACACGAAACGGAATAAAAAGATGTCAAATTAATTAGAATTAGAAAATTAAAAATGTTTAAT СтTAСтTTTAAATTAATATTTTTATTCTACCACAAGAATCTTCTTCAAATGATGTAACCAGGAGACTCGGT TTGACTGTCCTAACTAATATGAAAATGAATTTTAAATAACCATATTGCAGTTGTATCAAATATTCTAATTAAT AATTAAACTTTTTCACTCGCAGGGGTGATGTATCAGGAACGTGTCTCAACCCAGCCAGGGCTTTCGGTCCAGC TGTGATGGCCAACTATTGGAGCTACCACTGGGTTTACTGGGTGGGGCCCATTGGAGGAGGTCTGGTGGCCGCA GCTTTGCTCAGGTAAGAGAAGCCAGATCAGATCACGTGGTTGGACGAATGAGTGAATCGACTGATATGAGAGA TAACGCTCACAATGTCTCTCTTCTGTTGCAGGCTTATTCTCGGCGATGATAAGTTACGAATTGTTATGAAATC ATAACAGCCCCTGGTGgGTGTTTATTGCCATATAAACGACAATAAACCTGTAATGTCTGTCTCATATACTTTA GATTCATGATGTTGGATGCTGTACTTTTATTTAATTTTTTTCATGTCTATCACTGTGTGGATTTTTCAGTATG AATACTAAAGATGAAAACATTATGATGGTCTCTTCGTTGCTTCCTCTGCCCATTAGAAAACGCATGTAGACAT TATACTCTTACTGTTATGAGGAGACATAAAACACACAGTGGCAGTAGCAGTTTTGGgTGGCATGCAGTTTATT 
CTTTCATCTTTTGCAATGAGTTAGATGGTCGCATGTGGCAGACCGCGTGCCACTCTGTGGGCAGCTCAGCGGT ATTTCTCGGACAGAGCCAGGGCCACGGCGGTCAGGAATTTATCGAAGGCGACGTGAGCCTCAGGGGTGAAGTC ATTAGGGAACATGGAGGAGACCACCACGAGGACGCAGTGGGACAGGATCTGTGGATGGCAAAGGAGACAATTA GATAGCGAATATATGGTTTACACTGTAAAAACTTCAATGGAACAAGATAAGAGTGGGTCGATTCAACGTTACC CCCATGTGGCTTAAAATGTGGAATGCATGTGTGGTGCAAAACATGCTCCAAGTAAAATCTCCAAACTGGTGGA TCGGCATCAGAAATACAGATTTTTTTTTTTTAAGTCGTTTAAAAGAAATTAAGATACTACGGCTGCACTCGTT GCAGCGCCAATGCGTAATTTACGCGTAAATCAGACTATACTTGTGCGTGAAAGTACCCATAAAACCATGTCAG CAGATCCTGGCATACGGTGAAGGTAAGGTAATCTCACCTTGAAGTTGGTCGGGTCCACCCTCAGCTGGAAGGC GTGCTGCTCGCTGAGCTCCAGCAGGCCGTTTGTCAGGTCGTCAATCTTGGACACAGCCAGATTGATTCCACCC ATCACCTTCTTTCCGTGGGCCTTCACGGGGGCAGAGCCCGGGCTCATGTCTGGCCAGTGGGAGAAGTAGGTCT TGGTTTGCGGATAGACGGTGAGCATCCTGTGGAGGAGGAGATGTGGGTGTTATAAGTGGCGGGTATGTTGGAG AGCAAACTGAGAGTAAAACAAACATGCAGCCAACTGTATTATTTTCTTCCTTGGATTTTGACATGACATGCTC CAGTGGCGCAATCGGTCAGCGCGCGGTACTTATAAGACAGTAACCTGCAGAGTGATGCCGAGGTTGTGAGTTC GAGCCTCACCTGGAGCATCAGCCACTCCTTTGCTTGTGGACAGGTATGCTGACAATAACCAAATCTAAACTCT AAAACCCTCTCTAGTTCGCGTGTGTGCTTTAATAGTTAGTGTCTATGATCTGCTGCTTCTCAGTCAATTTTGC AATGCATTTAGATGTAGAGCAGCATAAACATTAGTTATAATTTATATTATCTAATGTCAGAAAAGGCCTACCT TTTTTTTTACAAGTAAACTAGTAAACAATTTAAGCAACAAAGAAACTGAACTTTTTAGTAAAACTGACCAAAT GCCCCTGTTTGTGCGCCCATCGCAGGCTGTGATATCATTGGTCTTAGTCTTTCGTGAAGTACAAATCAATGAT CGGCTGCAGAAATAAAGCAAAAGCATGCCATAAAACTACCACTGATATGAACCCGAAACGTGACACTTACCTG CTCAGAGCATCAGACCCGATGGCATCCGCGGACTTGGAGATTTTGGCCCACAGGGCCTTGACGGCAGCCTTGT CTTTCTGTGTCAAACTCATGTCGGTTCAGTTCTCTGTCTTTCTCTCTCCACCTGAATCGGACGGTGATCTCTG GTTAGTTTGGACTTGTGCTTAAATTCAGTTGGTTCGAGAGTCACACCCACGGGAACGAATGCCAAGCCACGTC CCCTGACGCAGATCGGACCCTTTCTCCCCGCCTGATAGAAACGAGTGGCCGGATGCCAGCTCTGAGAGGGGCT ATCAGCTGCTCACATCGTGGGCGACAGTTGGCAAAAATATGATAATTTACTTATATTTTGTAAGAGTCATGTG TGGACGTATGGTTTGTTAGGAGTTTCTCCTGAGGTAAAGCAGGGGGGCATCCCGGGTCACAGGGGGTGGGAGC AGACACTCTGGCACACATCAATAAACCTGATCTCAAACATAATCAAATTAATTTCCAAGTTCACTCAACCATT CCAACCATTATAAGTTGGGAAGCATGTGGCAGCCAGGGTTTCAGAAATACTGGGATTTTTAGAACAGTCTCAA CACTAATGCATTTTTAATATGACAATAATCACTTATAGGAAATAATTATATAATATAATAACTATAACTCGTA GATGGAAATTGTCCATCTGTTTTGAAATGACTTATTTACAGCTCAGTTTGTTATTGCACTAAAACCACTTTCA CTGTCAAACACACTGAGTAGAGGAATACAACTGCAGCTATCGTCCAGCTTTAGCCCTAAAACACATCATACGT GTTCTGACTCTCATTACTTATGTAATGAAGATAATGACTGAAAGTGTTCCTCTAGAAACGATTTAAAGAAGTT GTATCAAATTGTTGAAAAGTTATAGGCCTAATAAATAAAAATTATACCGATAGGCTACATAATACGGCCTTTC AATAAGAAATAAAATACTGTAAATGTAATTCTTATGTGTAATACAATCAAACGAAAAGTTATTTCTCATTATT AATCAATGATTTATATCGATAACTGAGGTATAGCTGAACATTTTGGGTCATGATCACCGGACACAGACTTTCT ATCTATAATATATATATATATAACACATGAAGGCAGCATTACAGAGGAACATCTTCAGCAGCACTCAGAAGAA ACTGACCTCCCTCTCTCGCATACAAACGCTCACATTCATTTAGGACATATATGCCCTGATGCTCAAGGCCCGG CAGGGGCCACAGACAAAGCTGCTCCCGTTCACTGTAACTTTATATTATGTTAGATTATTTCCCACAGTGAATA TGTCTGCCCCTGAAAACGAGCAGATACTCCTAATTGAGTCTGTGCGCAAAAGTTTGGGCACGCGTTTGACAGC ACATGTCCCCGTGTGTAACCGCGTAGATACGTGTGTTCAGTGTGTGTGTCAGGTGGAATGCAGGAAAGGTATC ACCAGGCCTATCATTTATGGCACTGAGAGGTTGGCTTTATAAATAAATCTAATATTAAATTATGATTTGGGGA TTATGACGCCCGTGTGTGCAACCTGTACTGTAACACAGTGCTGACTGATCTTGCAGTCAGTCTTGCCCCTCAC CCACCGCTCTGAACTGTACGTGCGCAACTCACACACTTAGCCCACGTCACATAGTGTGAGGTACGCATGAACG CAGAGCTCTGACGAGATAATGCCATAACACCATAACCTACAACTACAGTGTTAGCAATTCTTCACTTGAAATA TGAAAGAAATATACAGGATGCAGTTTATTGAAAATGAAAAAAAAAAAAGCAACATTTTCATAAAGAAATCATT TTTTGGATTTAGAACACTTTTATTATTTATTGACATGTTCAATATTTTTCATTCAAGTGTAAGTTCCTAGTTA TTTGAACACTGCAGGCCTGTACAAGATTTACGTTAGTAATGATTTTAGACTAAATGAACACAAGCCATAAAGT TCATTTTGGGCGTTAAATTTTAATCATACAATTTTCTAATAACATTTTATTCAACAAGCTTGTTTTGTTAAAA CACTACACATTTGGTGAAATTTCATTTCGACTTCGATTAATCATTTTCTAAAAAATTAATAATTAGTCTTATC GCGCCGCAGCTGTGGAAGCCCGACAGGATTTATCAGTGATTTAATAATTTTATTTTTAATCCGGCATTTTTGG CTCCAGCCATAAATGTATTTCCTATCAAGTGGAGAAAATAGCGCTCACACCCTTTATCACCGGCCTCAAGTAT TTTCTTAATCAAAACATAAGTGAATTCTGGCAGAGGGTGCCTGGGTGGGGAGGGGGTGCTGCTGGAGATAACA CAAGGGTGGTTTTATAGATAACACTCCACTGCTTGGCAAAGTCAGTGACATCTGAAATGTAGTCTAATAAACA CGCCCACACACACACAAAAAAAACTTTCTATATTTCATTTGAGACTCTAATTGTTTTTATACCTCTATGTTTC TATATTGTCCACACACTGGTTCCGATACAGGTTCCTGCATCATCCTTAATATCACCCCAAGCATGACTGAAAA GCTACTGCTTTAACTTCAATAAAGGATGCTTTCTGTAAAATAACGACAAAGTAACAAAGTCAGAAGACCTCAG TTAAGCAGTTCAGGGTTGGGGTTAGTTCAGACGAGGCCCATCCTGCAGGTTCAATTAAAGGAGCAGCAGAACG AACAATAGTAGGAAAAACTTCAATTTTAAAAAGAGTTGAATGTGCAGTTTGCCCTGGTTTTAGGTTGTGTGTG GGTGATGATTCATTTAATGTATTAATGACAAGAAAACGACCTAATCTGCATAGTTTTTCCTTCTTTTCCTTCT 
TCATTCTTTTTTCCTTCATACATGTTCCTTCTTTTTAGGTTTAGCCTGAGAACATATTGGACTAAACTTTAAC TGAGCATGAAAGTCGACTTTTGCATCTTTGTAAATCTGTAATACTATTAAACCAAAACATCAAAACATTCCAG AAACCTCTGGAGGAGTCTGCAAGTTTATCTCAGACCCTTCATCTGCAGCCTATCAAAGCTGGCCTCTCCTTGA GGGCGGGGGGGTCTTTGACACGTTTAAATAGAGGGTGGTCGAGCTGAAGCAATATTCAGCCTTCTTTTTCGAG CCTGAACAAACAATAAACAAGAGCCACCATGGTTGAGTGGACTGAGCAGGAGCGCGGCATCATCACCAGCATC TTCTCCAGCCTGGACTATGAAGACATCGGCCCCAAGGCTCTGTGCAGGTATGGAAAAACCCAGCTCCTGACAC CCCATCATGCACTTTCAGGACTTTATCCCATGCTGAATTATCGGTTGTCTCCTCCCCATGCAGGTGTCTGATC GTTTACCCCTGGACTCAGAGGTACTTCGGCGCCTTCGGCAACCTCTACAATGCTGAGGCCATCAAGAGCAACC CGAACATCGCAGCCCACGGCGTGACGGTGCTGCACGGTCTGGACCGGGCTGTGAAGAACATGGACAACATCAA GGCCACTTATGCCGAGCTGAGCGTCCTGCACTCCGAGAAGCTGCACGTCGACCCCGACAACTTCAAAGTAAAG CCTCTCTCACCTTCAGCTGTCAGCTCACACGACCTCGCAGAAACTCACATATGTTTTGGGATATAAAATGCAG ACATACCAAAGAGAGTTTCCCTGCGCATAATGTCAAAATGTATCTTCAAATCCTGTGCACACGCACAAATATT TAAAGTTTCAATATGCAGTTTTCTTGGATGCCAAATCTATTTATGTAGTTTTATATGAAAGGCATTTAATGTT TTTTTCCCTCTTCTTATAGCTGCTGTCTGACTGCCTGACCATCGTCATCGCCGCCAAACTTGGAAATGGCTTC ACACCAGAGACGCAGGCCGCCTGGCAGAAGTTCCTGGCCGTCGTGGTGTCTGCTCTGGGAAGGCAGTACCACT AAGCCCAGCAGCTTCCACATCTGGACACCCAAAGGAGACCTGACGTCTGTTTACATGTGTCACGTGTTTCCTC AATAAAGAAATAAAAGGCACACTGAAGCAAAGTTTCTTGGTTGTCCCTATTTTGGTTCCTTTATGCGTGCGCA ATTACGCACAGCCACACCGAGCTCCGGTGGTGATTGGGGAAGGTGCGAAGGAAAAATGACAGAGCCTAATAAG AAATTGCCACAATTACTCTATAATAAGGTTATAATGAGCTTTAAAAGAGTCAGTCATAGTGAATTTCTAACCT ACCAACCCACCGTGGTTTAAAAATATGGCATTTATGTATGTTAACGTTAAGCGCTGTGTATAGTCAGTGGCGT ACTCTGGCTGTCTGAGGGGCAAAAAACAGCTGCTTGAGCAGCAGCAGAAAGTTATGATGCATTTATGGTGAAA CAGTTCCAAACCCCGGTTAAGATTTAAATTGTTTTATTGTTATTATTTATAGATTAACCTCTGTAATAAAAAC GCACAGGGAAATATCAATTATCTAACCATGGAGGTAATGAATTATGTCAACTAATGTTATAGCACGAAGGGCA GCCTATGTGGCAGTGTTTTTGTTGCAGGGACAGTTTACGCTGATAAAACATTGCTGTAAGTGAGTTAGAAGAG AGGTTATTTTTCTCATGTAGTCCCTGGACGGATGTTACGGTTAAGTCAGAGGGCACTGAATAATGTATTACAC TTTTCTGTGTTTTTCTTTGAACCCCCGAGCCTACCAGTGGATATAGTAGATGGAGCCTTTGCTATCTCGTTTG CAAATCATTGGGGGAAAAAATTAAAGTCTAAATAAACGGTACACACGAGGATATTCGCACAATAACAGTAAGC GTTACTCTCTAAAGGCATCTCTTGGATTGTTGTAAGGTTTTCGGTGTCAGCTGAGGACAAAGTAGACTTGAGT CTTGATGTCGTGGTTTTTACGTGTGACGCTTGAACTCATCACGCACTGGGATTATCTGAGCCTGCAGGTCAGA ACAGATAAAACAGAGGGGTTCACCTGCTGGAACGCGGATCCGGCCCGGAACCCTTTGCTGCATGTCATCCTCT СтСтCTCTCTCTCTCTCTCTCTCTCTCTACTGTCACTAAAAAGACAGAAAAATATTTAAAAAACAAAAACAGA TAAGAGCTTGAACCCTGGGGGCCTAAACTATGACTGATGATTTCTGATCTTTACTTCATAAGATGTAAAATAA AACAAAGAGGACATACACTCAAACACAATTAAAAAACTTAAACCAAATATTTTGTTTATTACTGTAACAAAAT GTCTCTGAAACAAAATGAAATATTGTTTGAATTTTAGCTCAAAGACAGAAACACTAGTGCACACTTGTAGCCT ATACAGCTGATAGGATGAACTAGGTCTTGGTTTAAATTCAGCAACCAAGGATTTTTCTTCTCCTTTTTTTAAT TCATTAGACACTCCCAAAAGGCTGTGAGGTAGTACTGAACAAACACTGCGGTGCAAATGCTCGTTAAATGCAG CAGGTGGCGCTGTGTGCATGTCTTGATGAAAGTGAAATAAGTCTTGGCTTCATTCAACTTCATTGGAACTTTC ATCAATGTGTATTTGTAAGTCTAATCATGTTATCCCAGAATCAAACCAATGCAGACCTACTTATTCAGAGTAA ACGGCTATGTGTTTGATTAGCTTATTGACTAGTGTGTATCTTCTTGTGATGATTTCATGGATTGTCTTGACCG TTAAACTGAGCACGGTGAGCAGTTTTTAATGGAGCAACTAGGAAATTAGATCCCAAAGTTGTATGCTAAGAAT GTGACATTGCTGAGTCGTGTTCGCTGTTAATTTCAGCACCAGGGACAGCTCCGAACTCACTACAGCCAGTCAC TTTCAGCACCAGGGACAGCTCAGGATTCAATACAAGTTTATTTCAGCACCATAGACAGCTCCCTATTAAACAG GCTAATTAACATACCATACATTTCTAATAGTTTTTATAATGCAGAAGAGGAGAAATGAAAACATTAAGAGGAC AGATATCATGAAAACCCAACAATGTGCATGTTCAATCTATTTTCTTGAAACAGTAAGGTACAGAATTATACTG TAAAATAAACCATGTATCACCAGAAGGAACAGCTTTAATGTGGATGTTAACTTAATGTCTCCTTTTAAATGTG ACCCCAATGATTTATTAGGTGAAATTATCTTCAAGGTGACAAATATTCCTTTTTGTCGTTTTCCCTGTTTAAT GAATCCATTATTGGATTAAATACGATGCCAAACATGCTTCCACAGACTCGCCTGGTAGCAACAAGATGTGGAT GTGCAGTTACATATTACATTTCCACAATGACTACTTGCATTGTAGCCTTCTCTCTAATATATATATATATATA TATATATATATATATATATATATATATATATATATATATATATATATATATATATAGACACACACATACGTAA GTGTATATATATATATATTTTTTTTTTACAATTTACTGCTGATATGCATAAGAGTTTTAGGCACAACATTTAT TTTTGCCCAATTTTCCACTGGTTGGCTTGAAAAATGCTTAATCAAGGAATTTACTCATCATTATACACTTTAT GTGTAACAAAATTTAAGTTTGCAGTTCTTTTATGCTACACACAAACAGATAATCAAATTCATAGAATAATAAA AACATAAATAAAAGGCAGGTAATAGTGTACGTTCACTATAAGTAAACATATAAAGTCATATAATATGTCACTG CTTTAATAAGGAAAGCAAAATTTAAATATAATATATATAATATAATACCTCACATTGAAAATGTTTGACATGA AACTGAAGTAGTCCTGATTGTACATTATTATGTCTGACACATCTGCCAGATTGTACCTATTCATCTCATCATG AAGAGTTTCTTTTGGTCAGTCTGGAGAGCTGACATACAGTATGTGTCCCCTTCGACTTTCAACTGTGAGTGCT CTTGATGATAAAAGGCTGTGAAGAGAAAAATAGAAATACATAACTTCTGTAAGACTGGTAATAAAAAAATATA CATGTATATCAGCAAATTAGTTTTTTTCTCACCCGTTCTTTGGGTATATGAACACGCCATCACTGCAGACGGC 
ATCTTTCACAGGATCGTCCATCTGACACCACAGCCATGAACAGCCCACACTGTACGAGACAGAGAGGAGAGAG AACCGTGGATACAGAACATACCTACTGACAGCCGCAGTGTAATCATCTGGGTAAACCTCATTATAACTAACAA CAGGGACACAAATTCAAGTTACCTGCCTGGTCTTGAGAGACTTGACTGATGTCTCTCCGCAAACTCCTGCATG TCTGTCAGGCTGCACAGACAGGTCTTCTGGTGCCATACTTTAGCTCAGAGGTTCCTCGTACTCTTCTGGCTCA ACCTTTACTGTCACAATCTCTGTTTCCACAGACTCCTCCTCTGACTCCGACTTCACAGAAGACTCCCCTGGCT CGTACTCCAGAGGCTCCTGATATGACTCTGGGCTTTAACAAACAAGTGTAGAGAAGAGCAAATAAATCATACG TGATATCCAACAGTAAACAAAAGTGTGTGCTGTCTCTTGAGGTACCTGTCAGCGGCCACGGCACTCTCAGTCT CGTTGTTATAGTTGCTTGGGTCGTCATCAGAATACGAGCACGGGTCGTATCTAAACCTCTTGACTGGTGTGTC GTTTACAAAATCAAGAGAAAACTCAAGTGTAGAAGTGTCACATTCAACACTCCTGTGTGGGGCCCTGGCCTGT ACAGCTGTGAATAAACCACACAAATTAAATGCTGAGATTAAACAAAAAGAGCAACATTAGTACAGAGGTCCCT CGCATAATTGATTCAATTTAGTGGCCAATATGTTTTGTACGGTCAATTTTAAGGACTTAAAATGAGCGTTGGC CACAATGAGAGAACATTAAAAACACACCTGCTGGGATTAAATGCAGAATACCATCTGTACTTCAGTGTTAGAT TAATAAATCATCTAAAATAGCAAATAACAAAAGGGTTTTTAGTATTCCGGTGCCCAAAACTGGTGTCTTTATG AGTTAACCAGATTACGAACTGACTGTACATCTTACCTTTACTTCTCTTGAAGGGCTTCCTGTTGGCTTGTGTT GAGACGTTATGTTTTCCAATCTGAACAGTCTGGCACCTGACGTGGTTCACACATGGCTCCGCTCCACAAAAAG CATCCACGGTCCTCACCTCAGTCTGGCAACCCACATCTTTAAGGACTTTGGACTGAGGTAAACAGAATATCAG ATCATCGTAATAAATCTACAAAGTCACACAAAAAGGAGCCAGAACTTTTGACTCTTTCCTTGAACCTCTGGCA GAAAAGTGCCCGACATAATTAGATATGATAAGTTACAATATATGTTATATGTTATGTTACTTGTGCAATATGT TTTGTTGTTAGTATTTATTTTTAATGATAGGGGTATTTTATTCTGTTTATTCTGCCTTACCTCTATATGCTTT TAATTGTTAGGATTTTATGTTTTGCCTAATGCCTCCTGAAATGTTTTTAATCCTGGTTCGGACCATGTAACTT TGATTTAAAAAGTGCTGTCGAAATAAAGTTTACTATTATTGTTGTTGTTGTTGTTGTTGAAAGGATACAAAAT GCTATAAAGTTGAACGAAATGATAGAAGAATAAATACAATCCAACTTAAAAAGTTAAAGGCGCTCCAGCCCTG CTAAGTAAATTAGCTTGAGACTCATTTTCAGCTCTTACCGGCCGACCCTCCGGTGGATTTGGAAGCGCATCGT CGGTGAGGTGAAGCCGGACAGCGAAGCCCATCTGGGCCTGGGTGTAGTTGGTGAAGCACTCCGGGGAGAAGTG GAGGGCGCAGACTTTGAGCTTGGAGGTCCGAGAGTCCTCCGCCATGCCGAGGATCTGCAGCCACCGGAGAGCC CGCACACGGTCAGTCGGGAAAGAAAGCAGCTTCGTGGTGCTTTTACAACCCGGGAACGAACATGTCATCACCA TCCTGCGAAACCCTTGGAGAAGACTTGTGCTAACTAGCTAACCTAGTAGCTAGCTAGAAATCAACACCGCAAC TGTTGCTGTCACCAAAACTTGGACGAAGTGAGCCTTAACACGGAAACAACGATACAGTAGACTCATTTGGACA CGTAACTCTTAGTAGATAGTCATAAAAACACCTATCAAATGCACTTTTGTAAAAGTTTTAGATATATATTCCA TAATTTTGAACATCTATCGAGTTGGTTATGATTTCTTCTTCTAGGGCGGTCGCTTCCTTTGTATTTATTGGCG GTAGCAAAACTTCTGGCTGCATTAGCGCCACCCACTGGACTGGAGTGTAAAAGCACCACACTGTAGAAACACT GTAGCCTATTATAAGCAGTGGTGGTGACTTTATGCCCCCATTTTTTAATTTAAAGCGGCTGTCCAGCAAAGGA TCAACGCCCTGTGTTATAAACAGTGACATCTATGAGTACTTTTGTATATTTAGTTCACCCTAAAATGGGCCAG TAGTAGTTGACTTCAGGCAGTTAGGATCATTATGAATTTAACATTAGACACAGAAGATCAATACTGGCCCACA GACTACAGGGTGAGATAACTATATTATTATGCAAGTATTCCTTACTGCACCCACTCCGAGTCATTAACTCTGT CATGCAGCTGAGTATAATATTATCTTACCAAATGATGGTGCTGTTGCAGTATATATAACCCAGGTCAGTCTGC TGTTATTCTGCTGCTGTCCAGCAGGCTATACCATAGACAAGACCTGTGGGTTGGCCATGAGTTTGGCCACTGA TTAGCACTGCATATAAAACCCAGAACAAAGTCAAACAGCATGAATTTGATTTCTTGTATTCCCACAAACATTA AAATGCCCCAGCATTAAATTACTGAAAGCTCACTGTGAGGGATTACTCGTCTGAAGCAAGTTTTAAGTCTCAG AAAGAGAGATACCTTCAAATGAAAAAAACAGTCTAACTACTATATGGTATGCTTTAAAATTGGTCAGCAGTAC ATCATTTGTATAATAATGGGATGTCCTTCCAGGCTTTACAGGGTAATACGACGCAGCAGCAGGAAAGGTGTTA AATTGCACATTTTTATTATTACAAAACATTGCATTATAACAACCACACATACATAAATATTGTATTAAATATC ATACGGCATCATAAATAGTTCATATACTTCCTGAAAACAGGTGGCTGGCTCTCATCTGTATTTCTCTGCGAGC ACAGCTGCGAAAGCTGACAGGTACTTGTCCATTGCTGCGTGTGCAACTGGTGTGAAGTCGTCGCCCATGTGAC AGGCCAGGGTAACGAGCATACAGTGTGAGAAAAGCTGCAGAGAAGAGAATGGTTTGTTATTGACGAAGGACTC AATGCTGCATCAGCTGAGGCTCAGCAAAAACTAATAAAAATCTTTAAAAAAACATATTAGGATGTCAGTATAA TTAGGGTTTAAAGTGTTTTATGAGGCTTTCGCCTGCAAATAAACTAGTTTGTACAATGAGTCATGTGACCCGC TTAGACTGCCAATTGTACATTACTTTCCAGAAGTAACATGAAGCTGTAGTTTAAAGCATATATAATGGTGGCA TATTACATAACATGTCATAAAATATATGACTCCTGATTCTTTTGTAAACCTAATATTCTAAATGTAACTCATG TCAGTGTTGTAATTAAAGTCAGTTTCTTCTAAATATCTGACACTTATACTGTCACAAAAGCACCACTGACAAC CTAGTGACAAATTCAGATATAAGACTGAATACTCAGAAAACTCACCCCCAGCCCCACTCTCAATCAGATAAAC TGTCCAAAGTGCTCCAACAAGAACATAGCAGACACAAGGGAACCCCTGCAGCTGTGTGTTTATCAGTGAAGTA ATTGTGTTTCCAAAAGACCAAAAATCTCCTCTGATACTACAAAACAAAACCTTGGTTAGTGTCATTGGTAACA CCTGTGTTTACACCACTGTTTCAGGTCAAAATGGCTGCTGTAAAAAGGTCTGTTATATTATGTATATGTTAAA TTTGTGTTTTTATGGGAAACAGAAATAGATATGCTCTGCAGTAAAATGCAAAGGTGTATTCCACAATATTTCG TGTTCCCAGTCAGTATTGTATATCTCCATATACGGAATATAATGTTAAATGCACATTAAGCTACATGTTGAGA GAAATATTATAGACATGTTGGTTTTGAAGCTGTAAACAGCTGCTGTCAGTAGAGTTTGATCTGATATGGGCAT GAGATGATGTCATTTCCACACCACTGAAAACTCTATGAAGAACTTGTGAGTGTTATTATCACCATCAGTGGAA 
CTGATGTTCTAGTAGTGGCACCTTGAAGTTGGTCGGGTCTATTCTGAGCTGGTAGGCATGCAGGGTTTGCAGG GGAGCCAGGTTTACGGCCAGCTGGCTGATGTCTTGCGCTCCCTCTGCTATAGCCAGAACAATCTTTTTCCCAT GAGAGAGCAGGTGAGCGGAGCGAGGACTGATGTCTAGATGGGAGAAGTACGTCTTGGTTCCTGGGAAAGTGGC AAACATCCTGTGGAAGAATTGGGAAAAGTGCTGTAATGATCAGTGGAGGAATGTAACTAAGTACATTTACTCA AGTATTGTACTTAAAGTACAAATTTGAGGTGCTTTACTTGAGTATTGCTATTTTATTGTATTTTATACTTCCG CTGCACTTCATCTCAGAGGCAAATATTGTATGTTTTAGTCCACTATTTAACTATAAAGTAGTTCAAATGAGCT TAACCAAAAACATGGTCAGCAGTAAAATACTATGTACACATAAATGCAGCAGTAATCCAAAAACAAGTGAACA GTGAAGTTGCAACTAACTGAACACAGCTCGCGTCTGTATTTGACAACATGGAATGGGACAGGGGTGTGGAGTG TGTAGAAGAAACTATGTGAGAGGCCCTATGTACAACCAGTGTAAGGTAACGAAAACACAACAATTCCTACAGT TTGTTCTGGTGATTATACACTAATGAAAAATATACCTATGAACATTATATTCCATTTCTGACAACAGATCCTC CTCAATGTTACACACTGGACCTTTAAGTAACGGATCTGAACACTTTTGTCATTGAAATACAAAAACATTAGCA ATGGAAACTGAGTCTGGCCACTCCTTTAAGTGCTTCTCATTGATGCCATCATCATCATCAGTGGACTCACAGC TCACTCATTTGAACATTTCACTGATCTTGGATCAGTGCCAGCATCTAGAAACCACAACCTGAAGTTTCCGAGT AAACCATCAACATCTGAGTCATTTTAATTTACATGGTGCAACGCTCTCACATTGGATTCATTCATATAAACCA ACTGCCAAACCAATAGTTAATAAATAATTCACCAATGCTTTGTAACCATCAAGTCTGAGGTTATAAATGTCAC AAACTTATTAAAAAAAAGGTTTTAAAAAAACATAATTTTTTCACTGACGCATGCTGGTTCAGCAGAGCTCATA TAGCTCTTCATACCTATATTGAGCCTAATTGTTTGTTTTTTGTCATTGTGATTGAAGCTTTAACATTTACAAA CAAACAAAAACCAGACTTTTGTCTGTATCAGCAGTTTATTTCAAACCCACAGAAAGTTTCTATGTTGGAAATG TCTTATGAACCTACCTAAGAAGCGCATCTGACCCAATATCGTCTGCCACAGGAGTCAGTCTTCCCCATATTTG TGCAATGAGGTCTTTCTCCCTCTTTGAGAGCATTGCAGCCAAGAGAGTGAGGTGACTTCCCAGCGGAGGTCAC AGTCAGATTCACACCAGGCGACAACGATGTGTGCGTAAAAGCTGTTAATCGACCATTTTATCACTTCACTGAT GGCCGCTGAACGCAGAAACGGACGCAGAGTGAAAAGGAGATGAAGTAATAATTCCTTCCCTGTGTGGAGAAAC CGAGGTGTCTTGTTGTTCCCCCCTCCTCTGCGTTATCGGAGCGCAGCCGGGGCAGGGTGGGGGCTCCGCACTA CTGCGCTATCTGCTGCTGTATGTGCGTCACACTGGATGTTTCTACCATATCATTCATGTGGCTGACCTCAGTG CAGCTGCAGAAGGGGGGTTCTTCTGATGCTGCTCAACTTATTTTTGTGTATTAGGGATTATAGGGATAATAAT GTTTCAATAGGTAAAGTTCATTCTCATTATTTCCTTGAGTTATAACTGCTAGTTACTTATAACTCCACCTCTT GTGTATAATGTCTTATTCTACACTTTGACAAGGAGGAAATAGTCAAAATGTGTGTCAGAGGCTTCATTGCAGT AGATATATTGCAGTGTGTCAAGGGCTTGTTTCTGATGAAGTCTCTCAGCTGCTGCTATCTGCAGCTCCATAAG CTGCTGGACAGTGGGTGTGAGGCCAGCCTGCATCAGATAACCGCTTCTGTGAGGACCATCAAGCTGCATCTCC CAGTGGACTTTACCTTTTGCTGTAACACAATATGTGTCTTTTATTTATGTATTTCAACTGAGAGCATCCTGCA GAGGAGCCAGTGAGCGGAGCAGGATCAATAATGACTAGATTTTGTTCAACAACAACAGGATCATTTGATTATT TGTCAAATATCAACAGATATTAATTTAGGAATGTGTGAATGTCCACCAATATCAGCTGATATTATGCTCTTTA GTGGTACATATTTCCGAACAAGGTTACAGTTAATCACATTGTTACAAATGCCACATGGTGAGCCTGTCTACTG GGGGCCCCTGTGGGTCTGGGGTTGTCTGGAGCAAGTGCCCTTTGCCCCACATTCATGATCTGGCCTTGATTTT TAGGCCCAGGTTTAGTCTGGAGCTGCATTAAACAGGCTAGTGACAAACTGATGAAGCACTCTTTTGTCACTTG GACTAGGAAATGTAAAGACTTAGCAATGTAACATAATATATAGGCTATAAATGGAGCTGTGCAGTCGAATTGA TTGACTGACCTTTTTCACAGCAGCCATTTTGACATGAAATAGTAGGGTAAACACAGGTGTTACCAATTATACT AATTAAAGTTAAAGTACTTCTTTTAAATCTGATTTTAGGAAAACTGTATTATTGGATATTATGCATATGCATA AAATCTTAGTTAAGAAAAAGTTCACAGTATCACAGTCCCTGTAACATCCAACATCATAGCCCTCAGTTTACAG CACTGCAATCTAGCTGCTGCTGGGGAGGTAAACACAAAAACTGATTCAGTGTTTTTGAGAATTGAAACAATAT CACAAAAAAAAAAAAAATTACTGAGATACTCAAGTGAATCAATACTTTCTTTCCATACCATTCAGGTCAAACA GAGTCAGTGTGATGCCATTGTGCTGGCTGGCTGCTGTAAAAAAGGTCTATTCAAGCAGTTGCAGGAACAACAA ATGTCAGGTGTTTTTTATGAGTAGAAGGTTGTTTAAGGATGCCTCTATGAGAGAGTCAAAGGTTGGGGTGGGT AAGAGAAGGAAGTCTGTGCCAGGGAGGTTATCAGATGGGAACCTCCCATCACCCCCCAGCAGGCTCTGCAGCC ATTATGATGACCTTGCACAACCCCCCACTGTTACCCAGTGGAGTGATGAAAACTGACTCAGCATAACGAGGAG GTCTCATGTCCTCACATTAAGAAAACTGCAGAGTCATGCTGTTTTGTCTCAAAGTGGTCGACTGGTATGATAT AAAGTAAGAGGTAAGCATTATTAACCAGTACTTTGATTTGATAAAAAAAAAAATTCTATTGTATTTCACTCTT TTACATTACTGAACATTATTAAACTATTATACTCATTTTGTTGTTTTATATTGTATTGTATTGTATTGTATTG TATTACTTTATTAATCCCACAACGGGGAAATTGCCTTTTCACAGCAGCATTTTACGTGAAATAAATAAATACA GATATAAAAAAACTATTGTAAAGTGGTAAAGCTATGGTATGGTGGTAATTTGAAAATCAGAAACTACAAAATT GATCAGGTGTTGTAGAGCTGCTGTAGGAGCTACTCATGGACCCCACAGCGTCATGTAGGGGGTGAGAGGTGTT GTCCATGATTGATGTCAGCTTCACTAACATCCTCCTCTCACCCACTTCCTCTATGGAGTCCAGAGGACAGTCC AGGACAGAGCTCGCTCTCCTGATCAATTTGTTGAGTCTCTATAGGTGGTATAGGTTCAGATTTTATGTGTTGT TGTGTGACCTCACCTGCTGCAGGTAAAACCTCACTGCTCCAGGTGAGGCTTGAACTCACAACCTCGGCATCAC TCTGCAGGTTACTGTCTTATAAGTACCGCGCGCTGACCGATTGCGCCACTGGAGCACAACACCACTGTCCCAC TATCTTGATTATGCCCACACTACTGATAGGCCTTGTCAAGTTAAATTTGGGTGGAGCTGCTGGGAGTTTACTG AGGTCACCACCATCAGAAAAGATAAAGATTGAAGTTTTCTCTCCCCTGAACAGAATAAAAAAATTAAAATATA AAACAGGACTCCACAGCAAGTGCACATCCACACTGTCATCAAGTGGAAACAGCGGTGTAGGTAAACCAGGGAG 
GGTGTCGGTCTCTCCAGCAGTTAACTTTTGTTGTCTTGTGAACCATTTAACCGTTTGTTCCTTCCTTTTCTTT CACTTTGTGGAGCAGGGAGGGAGGTTAGTCCCCTGGGCGAGATGCTTTCACTGCATGTTGTGGTTAAAGGTGC TTGGACACTCCACTAGTCTGTCTATTTGCTGTGTTGCTGTGAAGCACTTGTGGTGGTCAGTACTTGAAGTTCT CTTAGTTTAAAAATCAAACTTTCTTTAAAAAGTGTTTAAAAGTAGTTATATCTATATATACAACATAAGGTTG CAATGTGTCTATATATATGTCCAATACAATAAAACACTTTGACAAGAGGTCATTCCCGAAACCTTAAGTGTAC TTTAGGTACATTGTGCTGCTAATACCACTTCCACTGAAGTAAATCATAAAAGCAGAACTTTTACTTGTAATGG AGTATTGTTATATTGAGGGAGTACCCACATTTACACTGATCCATCAGTGGGTCAGTTTATAGAACTTTATGAT GAAGTAATTGTTTTCCAAAAGCTGCTGTTCTCATCTGTTGACTGGAATCATACTACAAAAAACTGCCACTTAA TAAAAACTTCTTTATTACTCCACAAGGTGGAGCTGTGAGGTAGAGAGTTATGTTTCTTCTAATACCACAGGAT CTTTCATGATATTCAGATTCTGCAATATATATTTTGCTGATGTAGTTCATGTTAAATCAGTACTTAGGGATAT GGACTTAAGGATGAAGATATATGTTTATGTGTGATATTTCCTGAAGAAACTTCAGAATTCACAGTTTATTTTG TTTGTCAAACAAATAGAAGAACAAACAAGACTTATAATTACTCCGGTAAAATTCTGATCCAAATATTCTGTCA CAGCATTGTATATTGGTTTTGGACTGGACTTCACAGTGTTGTTAGCGGGTTAATTACATGCCTGAAGAAAGTA TTTCATATTTTCAATTTAGTGTAGTTTGATTTATTGTTGTGGCCACGAGTTACAATCACTGTAATCCATCACA GTGATCATCTTCTTCCTCCAAAGGTTACACTGTCATCATGTGGAAAATGACAGGCCTTTTTATTTTAAAAAGT CTGCACCACATTACCACGCTCAAACACCGCCAAAATAAAAACAGATACAGTCTTAAGTCGGGTGAATAGCTAT TCTACTATGTCCCAAGGCTCTGCAATTTGAATTTTCATATGGAAATAATGGATTAACATTTATAATTAATAAA TATAAGTTAAACACTTTAGTTTTTGTTAAATGGCCAGCACTAAGGTATACATGATTTGCTAAAAATGAGCAAC AACATGCAAAAATATTAGTATGGTCCTTTTAAAATAACCAACCAGTATATGACTGTATTACATCTGTTCCTAA TTTTTCCTCTGAACCTAAACCATTTATTAAAAAAAAGTTTAATATAACAGCAAGAATCAAAGACATAAGGTGG AAAAGGGTGATAAATTCCTCATTTGAATAAACAGATTTCTCTGTAAAGTCTATAAAAGCTTCCTAAAGCATGA AACGTACCTTTGCAAGTCTCTCCTGTTTTCTGCCGCAGCTGATTATCCTTAAAGCTTGTTTCCTGCAGCTACG CATTGTAATCAGTTTTTACAAAAGGAAAGCAGTTCTCTGTATGTCCTGCACGTAATGTGACTCTCTTTCAGTG TGCAACACAACAAAGAGCAAAGAGTGTTGCAGTAAATAGTATTTAGAAACAAGCCTTCATTGCATAAGATGCA TTTAGGCAGACATTTGTATGTACGTATATATGCACCTGTGTGAAGTGAGTGTGTGTGTGTATTGTAAATGGTT TAACCTGCCAAACATCCTGAAAGTTCGGCTGACAAACAATAATCTAAAACTGAAACAAAAATGCACCAAACCT CCAAAGACACTCGACCCGAGCACAGGCGGTTTCCTCCTGTCTTTACTGGTAACTCACATGTATAAAAGCTCAG GCCCAGTCCCTCCCAGCACTGGCCGGTTTGTCAGAAAGTTTGCCAAGAGTGTTTCCTGTGCTGGTCTCCAGGA CTTTAAAGCTGAATGTAACCGAGCAACTTGGACCTAAACAGGCAGAAGGAGGAAACACAGTCTCTCTGCAATG CTCAGGACACACAACAGACACAGATGCTTACCCATTCCTCACTTACACCTTCACACACACACACACACACACA CACACACACACACACACACACACACACACACACACACACACACACACACACACACACACTCACTCACACCAAT GATAGCTTTCTGGAAGGCTTGTCTGAAACTTTCCACATGAATCATGTATTTTTTTATTTAAGCATTCTTGTTT TAATTCAACCAGTACTTTGTGTTAAACAGGATGGGCTGTTTTGTTATTGTGTTATATTATATTCATTATATTC ATATAGATTTCTCAGCTCAACCCTAATATCAACCTAAAATAGATAATGTAACAATCACTTTGCAGAGCCCAAA TCACACATGAGGAGTGGAGTTACAAATGGCCAAAATCATGACAAACATATCAAATGGTGAGTCCTGTTTACCA AGCTGAGCAGTTATTGCCCCTGTCACTGAAGCTAACAGGTAAAGGTGGCACATGTGACTGACGACATTCCCAT CCCATGACACCTAGCCTGTAGTTTTCGCGCAGGTCTTTCTCACTTGCCTATGTAAGAGTGAGTGGCTAATGGT AGTGCTCCATCCAGTTACCCCACTCCCAGTGTACTACTAACAGACATTAAGGGGGGAAGCAATGCTCCTATGA TGCTATTAGTGAAAATCTCCACTTCTCCCAAGCTTGATCTTTCCCTTCCATATACACACTATGCACATTTCTG CATTATGTGTATTCAAAATTAAGTTAAAATACCTAAAGGTAGCAAGGTTTTTAGTGATGCTCCTTGTTGCTTG CAGAGATCAAAGCAGTAAAAACATACTGTATCTCTTTTACATATGTGCACACACACATCTGTTTCACTAGCAG TGGTAGAGCTATTGCAGAAAACCAGATGCTTTTTTAAATTAAACTGGTCAGAAATACAGATAAGTGTCTCATT СTTTCTTTCTTTGCCTTTCTTTTTTTTTAAGTGGAAATCAAGTATGTGATTAAACACTGAACTCAATGGTTCT TGTGAGATAAAGCAACTGGGGGGTAAATGTGATTTAATCTGATTGTTTGGGATTTCCTTAAGTGAATCTTAGG AAATCATTTGCAGGCATTTTGGCAGCATCCTTCCAAAGATAAACAACGTGTTCCTGCCAAAATGACTGCAATT GTTATTAATGATTCACAGAAGTAAGTACTGATGACTTTCATCATAATACGCTGGAATGCATTTGTGTAATTAT GATTTGAGTTTGCAAGTCATGTTACACCCTGAGCAAAGTAATGAGTTCATGATGGTACAGCCAGATGCACTGC CTGTATTTAAAAACGTCTATTGCCATAAGGCGTGAATTATAACCTTTAATTTTTCAGGTATTCATCACCACAC CAAAAGGGGAAAATCATCCCTACCACACCCAGAAACACCTGGCCAGGCTTGTATAGCGAACAATCTACAACCA CGAAAATGATCCTTTGTACCGAGCCGTATCTTTTCACATCTGACGACCACATTGTTGCAGGGACTGAACGTTG TGGTGAGCGGTGCTGATGAGTGATGGTGGTCAGATGAACCCTTTAAAGGTGGTTGTTGGCTGTGAGGTGGGTG AGGGGTTGAAGGAACAGGACACCGACCCTGCAGGGGAAACTGGTTCACGGGAATCCACAACTGCAGCACCGCC CAGCAATGCTGAGAGAGTCTCCAGGACAAAACATCAGGCTAAAGTAGACAGAAGGCACATGCAGCATTTCCAG AGGGACAAAGTGACACTGCTATATATCAATGCATGAAGTTTATTTAATGTTTTTTTTTTTTTTAAATGGGTCA GTTCGTATGTGTTTGTTCAGCATTTCAGTACCTAACTTTCACCTGCCCATGCTCATTTGTCAGTATGACAAAC AGTGTGGTTTAGGGTTAGGGTTGAGTAAACATGGAGGAAAACATTTACATATTCCTAATTAATATGTATTTAA ACTTGCTTCCCTGTTTGTTGGTCCTGTAGTAGACTAGTAATAATCATGTGTATTGTGTCTGGCACTTCTTTTC CACAGAGTTTGGTGAGTTTAAAGTTGCATTATCATTCCTGAGGTTATGAGACATCTTTGAAGATGTGTAATAT 
СTAAAACAGCTCAGAATTCTGCTTTAACTTAAGCCGCAATAAATACCTACAGTATCTCCAGTGATGAAGACAA TACTTCAAACTAAAATTTGCTGTCAAGAGCAAAAAGTGAGTAATACTTAAAGTTTTAAAGGACCAGTGTGTGG GTATAAGTGACATCTAGCAGCGAAGTTGCAGATTGCAACCAACTGCGGCCACCTTCCAACTGTGTAGGAGAAT TTACTGTGGCCAGAAAATGTAAATGGCTGTATCTAGAGCCAGAGTTTGGTTTGTCTGTCGAGGCTACTGTAGA AACATGGTGGTTCAACGTGACGGAAGAGGAATCACTCCCTCTGCAGATAAAAATGACTCATTGTAAGGTCACG AАAАСАCAACAATTCTTATTTCTAAGTGATGATACACTGATGAAAACATACCTATGAATATTATATTATATTT CTGCCAATAGATCCTCCTAAATGTTACACAATGGACCTCAAGAACTCAAGTATACTTGCAAACCCAGTGAGTA AAAGAAAAAATATCCACAATATTAATATTTTGTTTTAGATTAGATAGATACTATAGATGGAAAATACAGTAAA CAGATTACCAGTCTTTGCTACTCCTGAACTGTGTAAAACCTCTTAATTACAACGAACCTGTTTTGACAGGATG CACTCGCCCTAACTCTTCCCTGCAGTTGCTCGGTATCACCACTAGGCGGCACCGTGAGGTCGGCCGGCGGAGC GGCAGTCAGTCGGTCAGTGTCCTCTCATCCAGCGGCGGCGGAGGCGGCGGCAGCGGCAGCAGCGTGGGCGGAC CGGCAGTCACTCCACAGTCAGACCGAAAGTCTGTGCCGGTGAGGCGGGTGGACAGCTGCAGAGGAGCCGGTGT GCCTCACTCAGAGCAGAGGTTTAACTCGGCTGATATTAGACATCTTTATGGCACGCGAACACCGCCCTCACTA ACATCTGGAGCTCGCGCCTGATAGTGAAACTACTCTTCCTCGTGCCCGCGGGATGTGAGGCAGGTATATAACT TATGAAGTGACCGGCCGGCGTTAGCAACTCACCGGTGGAGCCGTCGGTGCCGTACTGGGCTGCAGGAACCGGA CTCAGTTTGAAGGTAAGCGAGGGGGAACACTGGGTGTATTTCACCGAGCCTGGTCGCCTCTCACACCGGCTGT GAGTCTGTGCACTCCTCGCTTTTTGTCGTTCACGGTGCAGGTTTTGCAGTGTGTTTATTTATTTATTTATTTA TCTTTTACCGGCGCTGCAGCACACTGGGGTTTCTGGGTGGGGTCTCACGGGGGGGGGGACACCCATAGGTGAC AGTGTCGCTTAATGCACGTTGGTCAGTAGGGTAGAGTTTTACACATGTGCGTTGGTTGCCTTTCAAATTAAAA CGTTCTTGTAATCTTGTGTGGTCAGGTTGTCATGACAGGAGTCAAATGCTGAAAGTGAGAGGAACAGGTGTGT GTGTTTGTGTGGTGTGTGTGTTTGTGTATGTGTGTCAGACAGAGAATAAAGCCACACACACACACACACACA CACGCAAGCACAGGGTCTTATTGCCAGGGGTAGCCTTAGATTCAAATTCCTCCTTCCCATGATTCATCTTCAT TTATTGATGTCTTTCTCTACTGTATGGAAGGCCAAGGCTTGGGGAGCTTTATCAATCTGGATCCAATGAAGTG TCTGTGTGTGTGTGTGTGTGTGGGGGGGGTTGAGAGTGAGAAAAGAAAAAGAAGAGGCATTCTGTATCGTTTT AACCCACTGGCAAAATCCCCTCTATATTTCACACTCTAAAATGCTGTAATGAATCAAGTCAAACACACACACA CACCCACACAGGTTAGAGAGATAAATCGATTTGAGAGGGCTTCAGGCTCCGGGGCTGCTGAGATGAAAGGGGG CACTTTCTTTTGCTGGCTGTGCAGAAATCCCAAAGAGACAATTAAACTACTGACCCGTGTCATCAAGGATTTG TTTTGGCTTCCCGTTTGGAAAGGTGGTAGGAAGCAGACTTTTGGGCCAGATGGCAGGAGCGCACATACACACA CACACACACACACACACACACACACATACACACACACCTGGAACAGCCAGTCAGAGTTCCTTCTTAGGGAGAC CGGATTAGTGGCCAGGGTTTGAACCTGTGGCCTGTTAATGATGAGTTAACTGACACACATATCAGTTTGTAGG TGCTTACAGACTCTGTTCTTGTACACATTACCAGCATTCCCGCCCTTTTTTGTAAGTTAGGTATAGAGTTTAG ATGATGTAAGTACTTAAATAAAGGCTCTAAATAGTTCCACTGTTGCTGAAGTGCCTACAGAGACTGTTAGCTG CTTATTGAATGTTTATTTTGGAGGGAAAACACAAGTCTTTCAGTAACCACGATTTGTTGAACACTGGAATAT TTTCTGCAAAAAATATTCAAATATTATAGCAATCTGATTCAATTTTGCAAACCAATACCTCTGGCTTTACATT CAGAGTGATTTATTGTTTTTACCAACCAAATCTCCAGTTGTTTTAATTTTGCATGTAAAAGCTTCAAGTCAAA GGTGACAATTTTAAACATCTTGTTTTGTCCGATCTACAAACTGAAGATCTTTGGGCCCAAAATGGTGTATTCT GCACTACTGAAACACAGCTATTAATATTATATCCCATTCCTGCAAACAGATCCTTTGAAATGTTACACACTGG ACCTTTAATTGTGATCTGAAAAGCAGCACTGCTTGTGAAAATCACTTCTTTTTTGCAATTAAACGTGTTCTGA CTTTGACACTTAAAAATTACAACTGAAAGTCTTTCTCATTGTCTGAAGACAAAAGTAGAACGCACTCAATCAC ACGTAGCTCTAAACAGGAAGCTGACAGCATCTCAGAAGATATCTCCTCTGGTGTGAACCATCGCTCTGTGTAC GTAGGTGTAAATATGGTAGGTGTGTGCGTGCGCGGTGACATTTTGCAGGTGTGTTTTTATCTCTTCGTCTTTT TCCTCTGGTGCACTGAGCGTTCTGTGTTCTGCGTGTTTGAACAGGCTGGATAGAAAAGAAGCGGCAGTGACAG TAGTGGGTTTAATTCATGGGCTGCTGCTCTTAGAACTGAAATGCACCTTCCTGTTTAGAGTCCTCCGTCGGGT GCATCAGGGTGGAAAACTGGTTTGGTTTTACAGGAGCCCTCCAGCATTATCACAATGCATTTTTCATGGCAGT TTGCCCACAAGTGGATCTTCTTCAGGGCCTACAAGCTGCACTGTTTATCTTTCTTGTGTTTTGTGCCACTACT TGTCACTGCGCAAGTCCTTTTTTCATCTAGGATCTGCCTGTCAAGAAATTCAAATAGAATTTTGACTCAAGTT TAACAAAAGCAGGAAACAACAGCATGTATAACTGTCAGGTTGTACCAGGAGATACTCTCAATATCTTACTCAG СTTTAAAATGCTGCAAATGTCTGATTTGTTCTACTCCTCCTAAGGGACATAACCCATATCCAAAAGTATTGGT ACCATTTTGGTTACGTTGTTTTCTCCTTTTCTTCTTTTCTCTTTTTAGGAATTTTGAATAGAAAGAGACGGG AAAGGCAAGGGGAGTTATGTCTTCATGTTTTGAAAGAATGGAGGCATTTGGACGACTGTTGAGTATTAAACAA GTTATAAGAATGTGTCTGCAGTTGTGAGTAATTCAACCAAGGAGAGTGTACTTTCATATATCTTTGTATGCT GACAGTCTGAGCTACTGGATACCTGAATTCTGATGTTATAATTGACTTACGAAGTTTTCATCAGTTGTCTTCA CCATGCTTATTGATTGACACTGTGGCAGAAACCACAGCTCCATCAGAACCACAGGTTTGATGTGCACACATGT GTGGAGGTTTCTGTGGTTATTTTGCAACTAAGTAGCGCTGGTTGCACAAATTATGGATTGACAGTGACGTCAT AGCTTCAGCAGATGTGCGTTCTCCAGCTGCCGCGGGCAAGGAAATAACTTCAGTGCACGCTATGAAAAGTGAA AтсCTTTTGCTGCCAAACAACAGACATCACGGTGCAGCTTTTTTGTCATTTACATCAATTGTATAATGCGTGA TTTGGGCACACTTTGAGATTCTGCATGAAAGATTAGCAGTGTCAGGACCGCTACTAGGTTTTTGGAACAGAGC TGACTGACAGGTGATGGCAGGAGTTATTGCGTCTGTCCTGCAGTTTGTCTGTCCCCCGAAAGACCAAAAGATC 
AAGATTTGAGCTCATCCCCAACAATCTGGACAGGGATTTCTTTAATTCTCTCTCTCTCTTTCTCTCTCTTTCT СTCTCTCTCTCTCTCTCTCTCTCTCTCATCATGTCTCTGGGTGTTTTTTTTCTGTCACTGTGTTAGGCTCACT CTGTATTTATTTATTCCCCCCCCCCACACACATCATCATGCTGTATCAAAACCAGAATGGCCCCATCAGCGTG ACGGATGCTGCCAGAGCACTCGCACAAATCAATATCACAAAAGAAAAGCTACAAAGCAAGTTCACACACGCAC ATTCTATTTTTACTTCACAGAACAAAAAAAAAAGCAAACAGTAGATCATCATATTTCAGAGATACTCATCCGT TGTATTGCTCTCTGTGTTATGCATATACACTTACAGTTACATTTGCATATACATTTACTAATCCTCTTTGGAT TCCATTAATCTTAAGTCACACGCTCACCACTGTGTGTTGCTGGTTTAATTTTCCTTTTCCAGTCTAGAGATAG AGACAGAAATAGGACGGCTGTAGGAGTCCATAACAGTACAAAAAACAGTGCTGTGCATCAAACGGGTTGACAA GGGTTATAGTGAAAGATCAGTGACTGTACAGTGGAAGCATGGACACTCGGGTCACTGAGTGGTCACGAGTGGA TCGTCAAATGAAGGGTTGATGAATAATGGGCAGATGTACTGTCATGCTTTTACTTTGAAATATTCACCGTTGC ATGAAAAGGGTTTGACTGTCCTAGGAATGAAGACTCTTTGAAGAGCTGACTGGCACTGGGAAAGATACTGGAT TGCTTTTAGGTAACTATTTCAAAGGTTTATCCATTACTTAATGCAGTGTATTGTAACCAGCTAACTATGTATT ACTTTAAGCTTGCTAACTGATTGCCATGTACTCTCAGTTGCAATGGTGTTCAGTTAGTACAGCTGACTCAGAT AAAAAAAAAAAAAAAATCAGATCAAATCATAATTTATGTTTTTCAAATTAGCTGCTCTTATGCAATCTTTCCA TGTGCCCCCTGGCAACTCCAAAATTATCCCCTGGTTGGAAACTATTGATTTGAACAGTTATCCCATTGTCTTC AGCAACACATTACACAATTTCTTTATCAAAACACAATTTTTTTCAAATGTTCTTGGCAACAGGTCCAATGAAA AGACCAAAACCAACTATGATATGATTCTACTAGTATTGGGTGTTTAGGTGAAGCCTTACCTAAACGGACCACA TTGTTGCCCTGGGCGACATGTTCCTTCACTACCATGAAAACATGCACTGTAGTTCATTTTGTGTCAACGCCAC ATACACTGCCCCGCCGCAGTACAGTCACTCGAGCAACAAATGCAATTAATATGCAGCAGGAGACAGTCCCTAA CAAATTTTACTCCCGTAAAACTACAATGTCCGGCTGTCGTTACATGCCGGTCACAGGTTGGGAAATCACACAG TTTTGAGAGATAAACTAGCACGCTTTAGTATATTCATGGTGTTTGTTGACAATGAGGAAAATATGGAGTAATG TCAGAGAGATTTTGTTTGTTAAACTTTTCTAATTTGACATTTTGCATAGGGAAATCCTTCAATAGAAACCCAT TGTGTTTTGCTTGAACTTGTAACAAAAAGCCTGTATGAATATCAAACTGCCTGTCAAAAAGGAATTTTAATCT GCCCATTATGTGGCATATACTGGTCTCCAAACTCTTCTTCGGTGTCCATTTCATGACAATGCTGTCCCAAGGC CAGTGTTCTCCTCAGAGACTCCAGACTTCTCTATGAAGATCCTGAATAACTCCTCTGTGTTTCTTTGAAAACC ACAATTATGACCTCCATTTTTCCCACTAAATCCTCTTTTCAGAGCCAATTCTCTCTCCCCCCGCTATCTTACC CTCATGTGGCATCTGTGGGCAATTACCATTAAGTTTTCATACAAGGCCAGCCAACAGAGGAAGCTGTTGTATT AGAGCTCGCAGCGTGAGTCTGCTGGCTGCGTAGAGGGACGGCAGGAAGGAGGGATGGCGGGAGGCATGGATGG CTGCTGAGGAGGTCTATTCATGTCTCCAGGCGAGGCCATACTTGAGTAGACACTTATTGTGACGGTGACCTTC CTGTCACCACGCAGCTAACACAAAGAAGCTTCATATGGAGGTCAGCTGAGATGCTCTCTTTGTTTCTCATCCT GCTTGTCAACTCTGTCTCTCTTTCCATGTCTGCTGCTCTTTACAACATTATATACTGTTTACTGTATACTGTT TGTTGAAGTCTCCTTATAATATAACAGCTATCCATTCTGAAGTTACAGATTTAAGCAATTTAGCATAACAGGG TTTACTGACTTACATTAGAAGACAAAGACGATTCAAATATAGTAAAACCTTGTGAAGTGACGGCTGACGGAGT TTATATTAAGCCTCACTATGACAAGAGGAGTGGCTGCATGACCTTTAAGCCTATGTGTGATAATCAGAGGTCC TGTCTCACAGCAAAAGCACGACAGTCAATCAGCCGTGTCAGTGCAGTTAAGTCCAGTGTGCGGCATTTGTTTG TACAGCAAACCAGTGGAATATTCTAGAGTGGGTTCAGTAATATTAATCTCACACTTGACTAGCGACCCAGGAT ATATAAATGTTTATTGTGACTGTCGAATGATGAAGCTACAAGTCAGTTTTTAAAAAAGTTTTAGTGAAATATT TCGCCACAAAATGAATCAGGATTTGCGTGCTTGCTCATAGCTTGTAGCCTGTGAGAGCAGAACATTGCTGGTT TAAATCCTTGGCTAGAAATGACTATAGTGGGAAAGTCAGTGAGAGATGCTTTCCATTCTTCCATCAATACAGT GCCTTTGAGAAACCTCCAGATCTGCTCAGTAATCAATGTAAGACTGTGTGAATATGTATGAGGGCAAAGCTGT СTCTCAGTAATTAATTATTTTTTTACTTGATGAAGAATAGGCTAAGTTTATATACCAGAGGGAAAGTATAGTA TATAAGCATGTAGTCAGGGTGGATGGGGATTGTACAAAGCTGAGGATTTACCAATCCCGTTTGAAGTTATATT TAGGTTAAAAAAAAAGCACTTTGGTCAAACATTGAGGTGATGATACCTGATCAATACGTCCAATATTCGCTCT TCTTTTAGCTCGGTTTTGGTCTCCACCAACTCCGGAGTTTTGTTTTTAAATCCTCCACTATGTTCACCAGCTA ACTGCTAATTTTGTGTGTCAGCTGTGTGGTGTTGGGCATGTAGCATATAGTCAGTTTTTAGAGCTATTTTTAT TGAAAAATATTGGTGCCGCTGGGAAGGATGCTGATAGAAGCATTGAACCACAACAGTGACATTTTGGGGTCGT AAAACCATAACAATGAGTTGAAAGATGCTCAAACGCTGGATTATGGACACGGATTATTGCTGTTTTATTGTTA GAGAAAGATTGCGGTTTGGGGTTAACTCGCGATAAAATAAATGCTTCCTGTGTCATGCTTAAAGTCATTTATG ATATTTTCAATATTTAACTTAGACCACAATCGTTGCTAAACTTTAACAAAGTGCTGCCAACCACAAAAAACCT TAATCATGCTTTAGTTTCCACATGGACAATTTGTATTGCAGATTGCAGAACAGATTTTGAAGGGAAAACAGAT TCAAAATACATTTTCAGTGAACATTTGGCATTATGTTGATGAGGAATGTAACGTGGGTGGCATTACTTTTCTC CTGTGATTTCTAGAAGACAGGGTTGTGGAGGGAGACTTTGCTCTGGCAGCTTTCCTTGGATAAATAAGTGTGA GTGTTTTTTGAAAACAGGGTGTTTCCCTATGACTCATAGGCTACAATGTTCCTTATTCTACAGATGTGGTCAA CATGTGGTCGATCAACTCCCCTGCTGTTATCTTTTCTGCATTTTGGCCCAAAACTGGTAGTTTGAGGGTGATA ATGCCTGTAGTCAAGAAACACGACATTGCTGCTATACTGTTCACTTTTGATTTAGTCATGTTAGACTTCAGTA AAAACATTGGTAGTACTCATAAAGACTTTAAAAAGACTTGACCTCCATTATATAAGCCATAATTATAAGTTGA GTTTTCCCTGTTTTGTTACAAAAGTGTTTTGGAGTTCTGCCTTTTCGGTGTTCTTCGTGGATGCTTTGGAAGT CTGTTTGTAGTTTTTGGCAGAACTGCTGCTAGAAGATTGTACATGGACTTCAACAGCGATGTACTCTTCATTT 
TAGAGCACTGGTTTGTCTTGTGCATCAGTGCTTCATCCCAGGGGTAGAAGTCTACCCTATACGTGCCATGTGA GGTCCATTAAACTTCTTTTTGCACCTCTAATATAATCTATAATACCTGGTAAATCTCCTCAGGGCACTGGGGA CTTGAGGACACTTTGGCCTCAATTACTCTACTGCATAAGAAGATAATGTTGAGAGGAGATATCGAAATACAGA GTGAAACTGTGACAGCAAAATATTCCCAGAACACAAAGAAAACATGTAACAAAAAGGAAATGCTTTCACTTTT CACAACACCAAGTTAAAAAGAAAGGACAGAAGAAAAGAGATAATTATTTTTATTCAGTAGTAAAAATAGCACG CAGGTATGAACTTGCTTTTCCTCTTTTGTGTCACTGTCATTGATATGCATGAGTGCTGTGTCAGCGCAGGTGT ATCTGTCGTGCTTCTGGAGGCCATTCTTGTTTTGATTGAGCAACACAATGGGTTTGGAAAATAAGAAAAACAA CATGTGTACACAGAAACAAAAAGGAGTGAGAGAGACAAAGGATATGAAGCATATGAAATAGCAACGTGAAAGG CAAGAACAGTTCACATTTCGTGAGAAAATGGAACTAACATACCTTCAGGCAGTTAATTGGAGGTAATCAGTGT TAGCAACGCTGAATGAACCTGGTTGCTTGGAAAGTCTTTGCTGTTTTTGGCCCGAAGGGATCAATTTGCCTCT GCAATAGACCTTTTTCACAGCAGCCATTTTGATGTGAAATAATAGGGTAAACACAGTTGTTAGCAGTGATACC AATTAAGGTTTTATTCCATTCAGGTCAAACAGAGTCAGGGTGCTGCTATTGTACATGTTGGCTCACTGGAAAG GCTCTGCTACACTAAATGGAACAGAGCATAAAGTAGTATAATTAGTAACACCTGTGTTTACCCTACTATTTAA TGTCAAAAATGGCTGCTGTGAAAAAGGGTCTATTTGTGCTAGTTCAAAGGCTCTTATTTAATAAGCAAACTTA GAAAGCGTTTTTTAGGTTAACACTGCAGCTAATTTGGAGCACATAAACTGAAATACAGAAGGGATTGTAGGAC TGCTGTGCTTCATCATTATTGGTTTATAAATCATTTTCATACAGATAATGCTGCATTTATGTCAGATGCCACA TTTTATATCTCTATTACCTTTTGATCGCTGTTCCTCTGCCTCTCTCTCTTTCATTCCCTCATCTTCTCTCATC TTCATCATCCTGCTTCATCCTGCAGTCTGTCACCAGCTACTACTTTATAACACTCGACTGGCAGATGGGAACC AGACATCCTCTCTGCTGAGAAAACTCTGCTCGGTCCTTACAAGTCCCATACCCGGCGCAGGAGCTGAGAAACC ATTATACTCTCTCCAGTGGCACTTTTGGCAGTTGAATGTGCCACGAGGTGTTGGAACAGTTGGAGTGACGAAT GGCAACGCGGGCTTTTTCTTTAGGGAGTTTTTCCATTAGTCATTTCTGCTGTATTGTCCCACAGCTGCCAGTG АTTTCTTACTGTTTAATCTGAAGAGGTAATAAATGAATGTGAAATGATTTTAGCCTAAGCTTTTACTGACATG TTTTTCTTATTTTTGTTTCTTACTGATTTTCAGAGAACTAAAAGCTGCTATAATCAGTATTATTGTATTAACA ATAAATCAGACGACTGAGTGTAATGTGAAAGTGGTCACTCGTAGTGACAAACCCACAGGAAATTATGACCCGA CCCGTTTTAGCATCTTTCAGTTCATTGTTTTGGTTTTATGGCCTGTAAGTGTACTGTTAGTTCAGTCTCACTG СTCTTATCACTGTTGCTTTCAGACACAGCAGGCAGTACAATAAAAGCAGACAAAGTTAGCAACTAGCCGGTGA ACATAGCGCAGCATTTAGCATCTAAAGAGACAGATATTTCCCTTCACAGAGCTAGAAGGAGAGGGAATTTTGG AGTTAGATTCATTGGGACACAAACAGCACCCGAGTCTGTAAACTGTTTGGAAAGCAGGTTTAGCACATCAAAT TGAAAGGTGAACATGTATACAGCTTGTTTCCACTGCCCCTATGTGACCACAACAGCCAGTTAGTGCAGGTTTA AAGCTGCTGTTTAACAATCTGCAGAGCATTTTGTAGTTGTCGAGAAAAGTATCTGAAGATATGTCCGAGCCGT TGTCGTCACACACGGACGGGAGGGTTACATGCACTGTTGTCTCTGCAGCTTCACCAAAACTAAACCTCCCCTT ACGGCTCAGGTAAACGAATGCAGATGTTCGGTATGAATTAACAAGCACAAACATGCATGCGTATACACACTTA TGCACACATAGTCCTGCACTTTTTAACCCTGCTGCAAGCCCACACAGTGAAACTATGCAAATGGGTGTCACAT GTTGTTTAGACATGCATGCACGAGGTAGGCAACTGTTTTTGACCGAGAAAGTAAATGTGTCTCTTCCACATCC ATCTTCCCTCCACGCAGTGTCAGGCTGTTTATTCTCTCGCTGCAATGGATTTCCTGTTGGTTTTTTTGCTCTT TCAGTTGGTGCAGCTTGTTTTTTTTTTTGTTTTTTTTACTCCTCCCTTTCTATCCCTTTGCTCCTTTCATCTA CTCCCAATATGACATAAAGTCTCTGCTTTTTTCATCTCTCTCCATCTGTTGTTGTATTTTGCTCTTCTCTCTG CCTTGATACATACCAGGTCATCTGCAGAAGAAGGCAGGAGGCCACAGCTCATGTTAACACTGTGGTAAAACAT TTCCAGCCCTAACGAAAGCATACAGAAGTTTTGAACATTTATATTCACCAGCACTGACCGTCAAAATTTGATG TGTTAGGACTGTTACAGGTGCCAGCAACAGTCGCCTGGAGGTTTTATTTGATAGCAAGACCTGAAAAGATCAT GCTCCTTTTTTCGTTACTTCTCTTCCTGTGCAGACTGCTTTGTAAGAAATGTGTGGGTGTGAAGTGACGTGCA GCGGGTCGGGGGTTTGAATATTGTGAGATAGAAAGCCACATGTCAATCTACAGTAGGAAACACTTAATAGCAG TAGCCCAGGCCAATCCTTTAATCATCTCCTAATGACTTCCATGGGAGTCATTTCTCACTGTGAGAAAGATCTG AGACTTATTATTATGAAAAAGCAGGAGTGGAGGAAGTTTTGGCTGTGGATTTTAACTTTCTCTGCTTTTGCCC TGGTTCCACGTTACCAATTAAATCTCTCCTGCTTAGACGGCTGAGTGGTAGAGTAGAGAAATGATTGCGCCTA GAGACAGACAAAGCATGTAGGACTGACCTCTGACCTGCTCTGATTGTCTGTGCTACTGTATATCTCTTAAGCA GCTTTTTTTATGCTGTGCCCTTATTTATTAAGGGCACAGCATTAATACATCTTTCTTAAGCGTATGAGCTTTA GATTCAGCTCGCCCAAATTTTAAAAACCACACAAATGATTATTTTTATTATGGGTTAATCTTACAGAGTGAAT GATGATATACTGTATTACAACCAGCCTGACCTCCACAACAGTGGTTCTGATGTCTATCATGCTACAGTAGGCA GTGAGTGAGGATGCATGGCACTTGGGCTCTTAACAGGAATGTGGGGTATTTCCTATCTGCAGTGGAGCCACAC AGTGAGGCAGGTTGGACACAGGTTGCAGAGCTAATTTATGCTAATTTATTATTTTAATTCTAGTACTTTGGTA AGGGAAACCAAGAAGTTAGCAACCACAACTTGCACAATTACTGATCATTGCATAACGTTGCAGAGTAGTTGCT ATGATTGCACAAGTGCCCTCAAGGTGAACTTATGTACCACACTCTGACCAACTATAATAGCAGATAGCATGCT ATTCCAATTAGCTTGCAGCTTACATTTAGCATTTAAACATCAATAAAACCATCACATTCATACTTACTGTAAA CAAAGGATACCGCCAGTGAAAA GACCAGGTTGTATTTGAATTTGCTGGGCTGTTTTATGGCACAGAAGAGAAC AGGGTGAGGGCACTGTTTTTCTACTGAAATGGATTCAAAGCTTTTTAGAGTTTCTCACAATGGCAGATGTTGC CAGGATTGAAAGGCAGATGGAAAAAACACTTTGAAAGTGTTCATCCTCTTCAGTAAAGTGAATGAGAAAACCA AGCGTCACCAGGAAGTGGGTAGGGGATACAATGCTTCAAAGGCCTTTTTGTGAGAGGAGGTACGAGACTTTTC 
TGGGGAAATATGGACTTAATTTCAAGAAACACAAGCCATGACAAAGATACTGGCTTATATGAAGCTATAAATC TCTCAATCAACATACCGAGGCGTCACATTTGACGGTATGCACGTATAAAAATGCTACTTATACAGTAGCTTTC AGTAGCTGCTTCCTGTGTGTGTGGTTTTGTATGCGTCTAAGTGGGTAGGGACTTATGTCAGCACCCCTGTGCA CTGATGTGTGTCGGGGCATAGGAGCACAGTGGCTGATCGACTGATGAATTGCTCACTCTCGCATAATCTGTGG TAACAAGATGAAAGACAGACGCCACAAGACAATCAAAGCAGCATTTAGACAGATGGCCTCAACTCATTGTGAA TCTGTCAGTAGATTATGGAGACTAACCAAGCTCTTTAGATTGGTGTTGGCATTTTGAATTAGTGTTGTATTGT GTAGTCAGTGGCTGTTACCTTTTCACTTGTTGTTTTGCTGATAAGACTCAGTCTTACAATTCCCAAATCCCAT TCTCACTTTCCGGGTATGGATCCTGGCAAATAGAATTGAACATCAGATGCCTGATTGAATTCATCTGCATATG ACTGCAGATATAGGAAGATCAGTGAGTTTGACTTTTCTCTGTAGATGATGTTTGAAAGATTACAGGAAACCGT TGATTATGTAAAAAGGATGACAATGTTGAGTCATGAAAACCACAGGAAGTCAACATATTAAAGTAGAATTTGG ATACAATATTAGCCAGAAAATAGATGAATGCTCCTCTGTTTTCTCTGCGGATGCCATTTATCATTCATCGTTT TGCTCTCTTAGATGATGATTTTCGTGTTTCCGTGTTCCATGGCAGATGTTTAATCATTTATTATTTGTTTGTA TAGGTAGCAGCAGATTACACACACACACACACACACACACTCCAGCAGGACTCTGCAAACAGAAAGGAAATGA CCCACTGTGTTATGACTGTAACATCTGGCACAGAGGAGAGCCTAGAGCATACATGGTCCTGTGGTGGGGGGTG GAGGGTGAAAGCACACGCCATAACTCACAGTTAATCTGTGACCTACTCATGCTTTGTTGCATCAGAGCAAACA GGATGATGGAGAAAAAATGGAGATGTACAGTTTTTTTAGGAGCTTGTAGAGGATTTCCATGAGAAAAAAAGAT TTGTAGGACAGATGATGACATATCACGTTCCTGCAGATCAGAAGCTTTAGATTTATTTCTTTATGCTCAATGT TAAGTTTTCTGCGTACTGGAAGTTGACATATCTTAAAATTACAGATTTAAAGAAAGAAATAGTCTGACATTTA GGAAAATACACTTCTTGCTGAGAGTTAGATGAGGAGATTGATACGACTCTTACGTCTGTTCTTAATATGAAGC TACAACTAGCAGCCGATTAGCTTAGCATAGCATTAAAGCAACAGAGATTTGTTAAATATGTACACATTCTGAA GTGTAAAGGTCTGTAAATAGCAATATCCAGCTAAAGTTTGCTAAAATTTGCTAACATTAGCCTCTGTGAGCTA CTGGTCATACAGGACCAAGTAAGGCTGTAAACGCATAATTCCTGAATCATGTATTATCTTGATTAACGGTGTG TTTAATTGCTCATGAATTTGACTTTTTATTTGTAAAAGAAAGATTTTTTTTTCTTTTAAAAGTTACATAATCT CACTTTAAACAAATAAGAAATTTGGCTTTGGCGTTGATAATTGGAAAACATATTTCTAACAAAATAACTTTGG GTAACTTATGCACGTGTATTTAAAGGAATCAGTTAGATCCCATGACCACAACTTCTGAAAGCACCATCATGAA AAAACTGCATATTAAACAGAAAGTTAAAATGGACCCAGTGGTGTTGTTGGGCAGGTTGTAGTGCCTATTAGAG CAGAACACTGGCAGTAATAAAGTTCAAACCTACCTGGACTGTTTGAGCATTAATCTGCCGCTGAGTCCCCAAC CAATTAACTTGGACGTGCACTGGTAATTGTCAGTTTTACAGCAGCAAGTATCTGGCTTACGTAGAGTCTGCAC AAAAGCAGCTAAAGTGACCAAGGTTATTTTGACTTTCTCTGGCTCATATACACCGTAAATCCTATCAACATAT CCTAATAAAAGAAGTAAGCTAACATAAACTTAATCCTCCAAAAAAGGTAAAAAAGAAATCACTCATTCCCATG AAATAAACCCAACTCAAAAATAAATTAATTAAATGACTGACTATTTTAAGGGCTTTTAAATTTGTTTAAAGTA TTGGTGATATAATTCCATTTTTTTCCATTGACCCATTTTTTTAAAGTTCACTTTATATCCGTACAAAAGGGTA AACCCCAAAGCCCCCAAATTAATTGGCTACCAATTTAATAAACGATTAATTATAAATTGTTTATTTAATCAGC TGTTATGGTAGTGTAATGATTTGCTTGGAAGAAGACTGAGTAAATTCAATTTAGGTGCATATGAGTGTAACAT ACTTACTAGTTTTAAGTTCAATTAACTGTATAAAATTGGTGCAATTTGCCCAAGTAGCCTTAATATTAAATAC TTGAGTTACTCAATTCAAATATTTTTGTTATGCTTACATGTTTGGAAGAAATACATTTAAATGGTTTAAGGCA ATCGGTTTCCTCAAACTATTTGAGTTAAACTAAGTCAATCAGGTTTTACAAGATAGGCTTTTCAATAATGAAC AGCTGTTGTTGCTTGAAAATTCAATGTATGTAAAACTGAAACAAGCAAGTAATTCAACAACAACCGTGTGGTA ATGACGGCAGAAAGTTTACTTGGCTGCAGAAAAAGTGATTTGCAGAAATCGGAAAGGCCCCGTTTGGAAATAC TAAACCACGTCAAAAACCACAACAGAAGCTCATAGAGCTTATGTGACTGACTAGCACTGTTAGCAGTTAGCTC ACCAGCTACAGTAGTTCACCAACTAGTCCTGTGAGTTGTCACAACGTCACCAAAAGCAAATAATATGCAAAGA TGCACTGATTCATTTCATTTCTGGTGTGCCTTTACACTGAAATGGTTTGATTCATTTATGCACTACAAAATAA AATAGCTAGCAGCTAGCTTGCATCCAAAATATTTATACTACCATACTACCATGAAAAAATAAAAAAAACTTTT CTGTCAGTCAGTATTCTGGCAGTACACGAGAGAGGTTTAGAGAACAAAAGGACAGAGAGAGCAGAGATTAGGT TTTGGACTGCTTAACATAAAGGTCTATTGAATATTAAAGCTTATTGACATGCAATCACAGTGAGTTTCCATGC CACAGTGACTGGGCGCCTCAGCCTCAAAGACCCAGAATCAAGAAGCAGGACAAATCTTGCTTGGGTGCAGAGG GGATGCCTTTTTTGTGTCTCAGCACGTCAGAAAATGTCACCCTAAAAGCAACCAATTACACCGCTGTTCTGCT GCAGTCCCCACATCAGTCTGCGGCACACAACGTCCACATTTCCTGTAATACTTCTCAAAAGGTAACAGTTTTC ACTTGACTGAATGAAACAGATATAGATAATCTACATCGAAATCTTGTCCGCTTGGTCAGTTGAGAAATTGACT TGCAAATAAAATGAGGATTTGTTTTGGACCTTTGAGCTGACTGAATCTTACATGTTTGTTGCCTCCTCCTGAT ATAAATCTGAGAAGATAAGGGGAGGAAGTGCTCAAGCTGTGAGCTTTAAACATGTGCATGACAACTAGACTGA TAAATCCCGACCTGAGTAAAAAAGACATTTCTTAGATGTTAAAGCTGGGTAGTTGACTCCCAAATAGTTAATT TTCTGATCACAGTTACTTTCACTTCCGCTCTGAGAATGAATACCTTGGTATCAGAGAAGCCACTTCCTGCTGG GTGCTGATGACACCAGCAATATCTTTGTCCAATCGGGGGTCAGTATGAGCCTCTGTGGCATGACAGCAGGGCG ATGTCATGTGGTGTACAGCGCAGGGATTACAGCCCCTAATCTGCCTGTCGTGAAAACCTAACTAGAGAAAAAA AGAGAGAGATCATGATCATGAAGTAACAAATGGCGGATGTCGGAGACAAGATGAAAAAAGAGGAGATTGATTT CGGACCAGACGGCATCTCTTCTTTAGCCGTATGGAGAACTACAAAAACCTGACGTGTGAGACTGAAGCAAGGG CTGTCAAGTGAGTTTATACACTCTATGGATGTGAATATGTATACTTTGTGCATATTCATAATTTGTATAAGTA 
CATCCGAGTGTGTCTGTGCTCCTGTCAGCATGCACACAAACACACACCGGATCACCACCACAGAATAAGCAAA GGATGTGGTTATGGATTGTTCTGGTGGAGGTTTCTATTTGCAGGGGTGTGTTTATCCTCTGTGACCGTAAGGG AACGCGCAGGCTTCCACATGTGACCACAGAGAGACCAAAACACTGACATGGGCAGCAGAGAGGCAAAAGGAAT TAAAGTCAACTTTGTGTAAGCTGCCTTTAGGCTCAGTCAACATGTGTAATTTAAACATTATACACCGTGGATC AACAGTGTATCAGTGTAATGCAATTCTGCGTCTCTTCACAAGGTGTTTGTTTAATCAGGACTCAGAGTTTAAA GCCACTCTGTCTACTTTTGTATGTCTTTATTGCATCTTTCAAATCATTCTGCTGGCACACGTTTTTCCTCCTT СтTTTTCTACAGTCATGTTAGCGGCTCTCTGAGGCTGTACTCTACAGCTAAATGCTAATTCCATCATGCCAAC AAGCTGATTTTATACAAGTATAATATTTGCCATACTCACCATTTTAATTTAGCACTTTAGCATGCCAAATTTG GATAATTATTACAATACACAAAGTCTTGTTGGTCATAATGAGGACGCTAGAGGAAGAGTTAAAGGTGGGGTCA GTTATTCTGGACAAAGATTGTTTGATTGTTGAGTCTCATTCCCGGGTCATAAAATACAGATGCTTGGCAACCC AAAGTGTTGGCGACCTTTGGGATCACCAAAGTTATTACAAATTTGTCACTGATATGGTGGCGCAAGAGTAATC AACAACAAACACAGTAAGTTTCCAAAGCAGTTGATAGACCAGCCAATATCGCTCTACAGACTCTACATCTAAA ATTCACAATTTTGCACTGATGTTAAGTGCAGTATCAGACACTGTAGAAGAGAGAAATACAAACATCCACACCT CCAGTAACCTCAGTAGATCAGTATGTGATGGAACAAGCTGTGATGTGTTTGACAGTTTCGTTTAACTAAACGG AAACACGTGCCCAGCTCGTGCTCTCGTTGCTCTATTACTCCCATCCATAGCTGAGCAATGTCACTTTGTATAT GTCCAAACACCCACCTTTTACTAAGACTGAGAGTCACAAGTTCACACCAGTTCTGTAGGCATTGCTGCACAGC CACCTGGGACATGTAGGAGATCACTATTTAAATTGACATGGCAATTTGCATGAGAGCAAGTACAATAGAAAAG AGAATACCATTAAGTGCAAAATAAGCACCCAAACTAACTGTATTTGACAAGTAAGAGTATCCAAAGTGAGATC TTTCCCTTAAGGATGTAGTGCAAGTGTGTGGGTCGAGGTTTGGAATCGAGAAGTCCTCCAGCTGCCGGTGTTG AATGTCTTTCAGCTCGTCAGACTGGCCAGAAGTCTGAATGTCGGCTCCAGCACCACGTACGATGGAGGAAATT ATTGTTCAGCTGATGGCTTTATGGCAAAAGAGGAGAGGCTGTAATTTCACTCACAGCAGTGAATCAAAGAATG ATTCACAGCTGAGATCTACAGGAGCTCCTCGATGGTAGTCGCTCAATGCATCTCAGAGCAGATGCAGATGTCC TATTATCCAGTAAATAGCCCTTCCACTCCTGCTCAATGACTTCCCCTTGGCCCAAGAGACATTTTTTCACATT AAATATAATTTGATAAAAGTGAACGGGGTGTGTAGACAGATGATAGAGAGAAACAGACCTGCTATAAAACTAC CTGAGATTTCTTCCATTTTGCCTCCAAGAGATGGAATGTGCTGAATTATCTATGTTTATTATTTGTCCTGGAG CCTAAACTGACTGAGGTAGATCGATTATCAGTCAATATTAGCCTATTGTTGGCTGGTATTTAAGAGTTAACAG TAGAGGTAATTTAGAAACAATGTCTCCATTACAGATGCTGTCCATTGTATTTTGTTATCAGGTTTTTAATGAT TTTTTAAAGTTGTATGTGTGTAGCGTGATGGGATGGCAGACTTTCACTTCGTTGTGCAACCTTGTGTCGATCC TTGTATTGTTATTGGCCTAAAATAAAATTCAGTATGGGCTCTGTGTTGGTGGCCGGGGGATGAATTTGTGGTG GTGAATTCCAAAGCCTCAGCGCTTTATGCTATTAAGTGAGGAGCTATTCAGTCATGATAAAGTGCTATTGTTA CTGTGTGGCACCGGTGACACAGTAAGCAGCAGAAAGGCGCTGAGTCGGTGCTTCACCTGACGAGCTCAGCACC TTTAAAATGCACAAGTCAACGGGAAAGGAAACGATCATTGTTTGTGTCGACACTTCGTACCACCACTACAAAA ACTGCCTCACTGGCTTTCATAGAGACGGAGAGATGTCTCATTGACTTCCACTGAAAGAGTTAAGCAGGAAAAC СССTTTCACTCTCCAAATGCCTTACTTTACCCATAAACCCATTAACCAGCAACAACAGGATAAGTGGAGGCTT TGGCATACTGTTCTGTGGCTTTCCCTCTGTTTGTGTAGCTCAGTTGTTCGATCTATCAAGCTCTCTCTGTCTG ACTGTGTCTGCTAGGTGGCCCGCTACACGCAGACCACTGATCCTTTTATGAAAAACACACTCAACAGCAAGTA ATAGCATCCCTTTCTGTTCTGCCTGTCCCCGTTCACTGAGGGCACATGTGCCTGTCTGAGGATAGGAGGGGAG CCTTACCCATGAGAAGCCGAGCGCCATCTTGTGGTGGTGAAGCATGGTTACACTACTGGCCTCGCAAAACACT ACTGTATTGTCTTCCCTGCACAGGCTCCAGTGGCGCAATCGGTCAGCGCGCGGTACTTATAAGACAGTAACCT GCAGAGTGATGCCGAGGTTGTGAGTTCGAGCCTCACCTGGAGCAGTGAAGTTTTAACGTGGGCTCTCACAGGA GAAGGTCTGACAACTTGACATCAGTGGGAATGAGATTATTCTGCAGTAATCAGAGCAGACAGCACTTGTCATT GTTGAAACCAAACAGAAAGCACTTCTGTTCCTCCTTACACACAGTCATCCCACCTTACTGTATATTGAATGCA TTTGAAATATTTATATCTCACTAAATTTACACATTGTGAAATTATCAGCTGTTTCAAAAAAGGCCTTTTTGTG ATGACACCACATTAAAATATAAAGACATGTTCTTTTTATTGGGGAAAAGCCCATTTGGCTCAATCCTCTTCTT ATTTACCCATGCTGTAAACCACCAGCCAACTAGCTGTGGATTTGTAACAGCTAGTAAGATCACTGCTTCATTC CTGTTGACATGTCAGAGCATCAACTATTAGTTTTACCATTCAGGTGTACATAAAGATATTTTATGTAATCTAG TGGCCACCTGGTAGACAGGTATGAGATTCATTGAGAATTAAACTCACAATAAAAATGCATGTCATCCCCCCTT TCCTGTCACTATTGATTAAAGCCCCCAAAAATATATTAAAGAAAGACACGTTTAGTGTCTATGGTGTCACACT TTTTTGGAGTAGATGCTGAGGGTGGTTGGCAGACTAGTAATTAGGATTAAGCTTTTCCAGGCATTTGCCTCCA CGCTGTATCTGTCTGAGCCCAGACCATGCAGAGACATGGATTACTGGTGATTTTGTGCACACTCTGTCCAGTG TCTGTAATTACTCAACAGAACTGGTGTGTTTTGCTTTTGGGCTTTTGACGACATGTGTCTGTGAACATACTGT ATTTTGGCTGCGTGCGCTGTCCCACAAACCTATTTCTTTTGGTTGTATCCTCCAGCTGGCAGGCGTAGGGGGA GCTAGTGAGCATGGATGAGCCGGGCAGCAGGAACAGCAGCCTGCAGAGGAAAAAGCCTCCATGGCTCAAGCTG GACATCCCAACCATCCAGCTGACGCCTGACGACGTGCCCACACTTACACAGGTAGATACACAAGGGTAAAATA CAAGTCAGAATAGTAAAACTGTAGTCACAGTAGTTTAGACGAGTAGAGGAACTACTAGAACTGCTTGTACTTG TTTGTTTCACATTCACATACTAAGGCATCATCTGTTTGTGTGTGTGTGTGTGTGTGTGTGTGTGTGTGTGTTA CAGCCAGTAAAGCGCCTGCGCAGTGTCAGCATGCCAGGGGAAAACCCTCAGACCTGCATCGCTGCCTTGGAAA CTTCCAACAACTACCTCAGACCTCCTCTGGAGAGATTGCCCTCCTTCACACAGTCCATCAAAAGGTTAGACAC 
ACACACACAAATAGAGATAGATTCATAAGAGGTTCTTCTTTCTCTTCTTTCCTCTGCTGATTTTCTCCAGCTG CTAATTATCTTCAGCGTGCCTTATGCTCTCTAATTCTAATAACTCTTCTCTGCTAATAACTCTTGAGCAAAAC TAATTAAAGCTGCAGCCTCCTTACTGATTTAACACAAACTAATCTTGCTTTTTCTCTTTCTTCTTTTTTTCTT TTTTTTTCCTTCTTCTCTCTTGTCCTCCCTTTGTCTTATTTACCATCGCTTTCCCCCCGCTGCCTCCCTGTCC TCTTCTCTACATGCTACTCTATGTTTGAGAAAGTGAGAAAAGGGTGCGCTTTGAGCGGGTCAACACAGTTCCT CCAAAGGGCCAGAGGGGCCCCAGGAGGGCGTCAACCGTCCGAAGGCGGTCCTGCATCCCAAAAATACTGACCC GGCGGCGTTCATCTATACCCAAACAGATCATCAGGTACAGGAGAGGTGGAGGGAGGTGGAGGAGGGAGCTGAT AACTGCGGTTTGAAGGTGTTGGAAGTGGACTTTCTTTAAACTTGTGTGTATAAGCTAATACACAAGACATCTG GATTATTTTAAGACGAGATCCTTAATAGAAGAGATTTTATCACAAGACTAGAAACACAGAAAAATAGAAATGA ACTGTCTGGAGAATGACACACAAGGCAGGTTGAGTTAAAATGAATACACCCAAACTATACTGTTTTTTTACAA AACTTTTTCGCTCTTGATGTCTGTGTTGACTGAGTTTTACAAAAAGGTCCTGTTAGCCCTCTGTAGTCTCTTT ATGCCACAGATGTGACACTTTGAACAATGTCTGTGACACATGAAGGCTCTCGCACACACACATTGTATCAGTG TCAGTCCAGGTGGCACACACAAGACCACATGCTTTGAGGCAGACAAAATAATACAATTACTCATACAGACTAT CTATGTGCCTCACACAGCATGTGGACCACATCAAAACGGCCACTCACGCAGCGCTAAATTGAATGTAAATGTT TGAACACGGAGCCAAGACTAAAACCTTGAGCCACTGTCTTCAAAAGAGCGTCTTAATGTCTTAACTTTATCAT GCCGAGGTTTACTAGCCTTGTATGTGTATGTGGGTGATAAACAGAATTAGTACGTTTGATGTGTATGAGAGAA TTAATGGGATTGAAGGGCAGTTTGTGTGTGTGTGTGTGTGTGTGTGTGTGTGTGTGTGTGTACATGACTTGTT GGAGGTTAGCTGAGGTACCTCCTCCTCAGAAGAGTGCATGAATACAGGATAAATCTTTAGTCTACAAACAAAG TGTATGTCAGTGTGTGATTTGCTGCTTCTGGCTTATATGGCAACTGCGGGGGAGGACACCCTGATGTTTGTTG ATCTGCAGGACAACAGAGGAAAATCTTCTTGAGTCATTTTTCATGTAACCAGTAATTTGAACAGATGGGCTCA TAGATAATAATTTATCTCAGTCACACTGATGCAAGCAGCGATAGGTGTTTGAACTGAACTTCACACGCTCAAT TGGACTTCTTTTGAATGCTGACACAAATACATTGCTGCAAAGAAAGGTGGGGTTCCAGCTAAACCTTTGTGGT TCTACCAGTCCAGTGTGTGAATGAGTACATGTATGTCATGTCCAGGCATGTCCCAGTGCCGTCCCTCAGTCTC TCCTCCCTCCTAATGTGTATGATGTCCCGTATCTCCCTCCTTCTAATCTCCTGTTTCCTGTGGAGTGCTGGTG TTTACACACACACTCAATGCAAACCCAGGCAGAGAAAGAAAGACAGAGAGAGAGAGAGAGAGAGAGAGAGAAG GGACCTCAGTGATAGAGATCGTTGGGGCTCTGGAAAGGGGCAATATTCAGTTTGACATGGACCAGAGCTGCTG TCTTCTTTGCCCAGTAGGTCACATCCTTATACGCAAACGCTCGAGGAGGGGTACTTGGAGGCCTGCCAGCCTC TGACACATTCACAAACTTTCTCACACATACACAGTCATATGGAAACTAGTATTGTGCGTTGGAGACACAAGGT CAGAAAATTTAAGAGCCTCCCGCTTGTCAGTGCTCTGAATGAGCGAGTCCAGCTGGTGGAAGACTGGAAGTCC TCTGTGGATATCCTTACATCAGGGCGAGATCAGAATCCCCGCCAACGAGATCATCGTCCTCTAACCTTTGATT AACCAGGAGCTCGCTGAGCGGGACAGGGATGTTACACACAGTCTGGACATGATGTAGCGATCATCCTCTTTTT GCGTAACAGTCGAGTGTTACAAGGCACTGCTGGCCTGGATATCGGGAAATCCCCTTATGGAACACTACATGTG TGATCTGCAGCGGATCAGTTTGCATTCAGCGGATCAGGGTTTAGTACTGGTCAGGGGGTAAGCGGGGACAGTA TGTTAGTGATGTTGGTGATGGACGCTATACAGCTCCACAGTCCCTGCCCCCCTCAGGTATGACCCAGACCCTG AACCCCTCCTGGAGCACCCTGGACAGGTGAGTGTGCTATGGTTGTGCAGAATGGGGCAAACCAGGGCAGAAGA TCCTGAACAAATATGTTGATCTGAGTCAGCTCGAGGCACTTTGGTCAAAGGATGGAATTGCTGAATAAAGCCT TTGTATTCAATTTATTGTGGAGGGATTAAGGTTATTTTCTTAATAAAAAAACAGAAGAATTAGCTGAGACGGG CATCTTATATGTTAATAGCCTTGAGCGTTATGTTTTTAATGAACTAGTGATTGCTAACATCTACATGCAACAC ATGTGATATTGCTGTGTTTTTATTTTTGCATTCAGGAAGGTAATAATCAGATACTTAAAGGAATAGTTTGACA TTTTGGGGATACCCGTATTTGTTTGCTTACCAACAGTTAGGTGAGAAGACCGTGAAGAAATCTGCTTACCAGC AGCTGTAAATTTAACGTGTTACAACTTGTTTATCTATACAAAAAGTGATGACAGGCTTATTTTCTTCAATTTC AATGGGGCCAGCTGCCTGGCTGAGAGCTATCAGTCTTCTCAAGTAGCAGTACCACCGTATAGACATACTCTAA AAGTGACAGTCCTGTACATACTTTTATTGACAGTATTAGCAGAGCAGCAAAATGTACTTAAATTATCAAAAGT AAAGTACTTGTTAATGCCTTAACTGTATGTTAAGGCATTATATGTTGTAGCTGCTCCTTTTTTTTTACTATCA AGTGCAATCAAATTGTATTTCAGTATAGTACTTGAATAAATGTTGTTCCACCACCACTGTCTCAAAGTACAGA CTGTCTCTAGCTCAAGTCACTACAAGCCAATATTTCATGACAGTTTTTTAATTTGGATAATTTTTGTCCCAAA AAAAACTATTTTCATGTCATTCCTTAAGGAGTTTTCTTTTTGTCAGAGTACGAAACAGCATCTAGCCATGTTA CCATGACAATAACATGCACCATTTGACCCAGAAAAATATTATTATATTATTATTTTTGTTCAAGGAGACCATA TCTGTTCAGTGATCCTGAACCTGACACTTCTTCCCTTAATTGAAGAATAGTAGTGAAGTAGTAGTAGTCTGGA AAGAACAAATGAAAAGTTAAGTATGTTTTTGTAGTTTTTTGTTCAGCTCATTTCTGTTACATCAGCTATGGAC ATGTGACCACTTATATTTAATTTGCTGTTAATTAACAGCAAATACTGGTAAACTTATATATCAGGCTAACCCT ATAGATCTGAACTCCAAAAACCCCTCATGTTGGTGGATCAGAAAGTGCAATGCCTATTATCAGTGTTCATATT TATCTTCTATATCTGTACAATTCTCTTGTTTGTTCAGCCATAATATTTGTTTAGATTCTAGATTTGCCCCTTG GCCTTTTCTCCCTGATAAAATGAATGCTGTGTAAGCCTATAATTACAGTAGAGTCGATGGATACTTTGTGTGT ACCAGCCTTACCTTGAGGATATGAAAGCTAATTCATGTTGCTTAATGAGGCTTTAATCTTTGATGGCTGATGG ATTTGTGCCGTTGTCAAGCAGTTTTGAAATATTTTATTTATGGTTTTGTGTGGGCGAATATGACCCGCATGCT TGTCGGTGCATGTGACAAATACCCGCTTACCTAAACAAACACACACAAACAAACGTCATCTATCTTGAAACGG GCCTTGATTCACAGTGTGGACGAGTGTTTGCATCATGCTTTCCCATCAGTAGTGGATGTTATGTTAATCTTGA 
TTGTTATCTGTATTTAACAGTTAACCAAAGTGAGTTATTGTTCCGTTATCGTCACACTCTGACCTCCTCTTCT CCGCTGTCTCTGTTTACATCTCCTGCTCAGAGAGGCCAAGTCTGACTCCCTTCTCGGCTTTCTCAAACCTGTC TTCTTTCTAAGAAATTCCTCTCTGGAGGGACAACAATCAGGGCCACGCCACCCCCTCCCTGCCCCTGTGCAGC CTTAAAAGGCATCCCCAGCTGGCGTCTTTCTTGATGATGAAGCTCGAGTGTTTATTACTGAGCGCTCTGCTCT CCAAACAGTTTCCCCAGAGAGGCCCCCTCCCCGAACGCACAGCCTGGCACGCAGACGCTGTTTCATGGAAAAA AGGGACCTGGATGCACACACCGAATGCACATTTATTTAGTTTAAATGAGAAACAAACACACACAGCCATGTCA GGTCATCAGCTGCAGGACTTTGAGGCAACATCTGCTTCTTTGCTTCTGACAGAACAAAGGGCAGTAGGTTATG TAAGGATAAAGACAGTATTTTTCCTTCAGCCTCTCTAAGCTGCACACATAAACAGAGAAAGCTAAAGATAGGG GAGAGAGACAGATACAGGAGGGGAGCTGAATTTATGAGGATTTCTCAGTCTGATATGTTGACATGTTGCACAA CAGACCTTTATTTAATACACTGTCCTGCTGCAGCCCGATCATAGTCCTCACTGTGTACTGCGGCTGTAGAGAC GCCGGCCCATATTTTATCTTGATTTTTCATTATCCAATCACTTATAAACTTTATCCAGAATTACGTTTAATCT GATCCATGCTACTTAAATAGATCTAATTTGATGATTTTTCCATTCACCCTACATGCCCCATTCATCTTTCTTT TTCTGTCTCAAGTTTCTGTTCTGTGGCAGCTTAAAATTATGCTTTATCAGCTATATTAAACCACACATGTTGG CTTTTCCATTTAGTATTTAAGGTACCAAAGAGAAGGTGTAAGGCTCCATATTTAAAGAATGCGAGACCAGATG CTCGTTTGAGATGTACGGTTCTCTTCATCAGTATGGACAAGAAAGCAATCAACCAATGAACCATTTAACTCGT CAACTAATCCCCAACAACCAGCTGCAACCAATCAGCTAATCAGCATATTGTTGTGCTAATCTGGCGGGATGTA TGGCTCAGGCTGTCGGACAATACGGTGCCTGTCAGGGTTTTCTGAATGCTGCGCAGATTATAGACAAAAACAG AAATAAGATAAAACAGACGACAAGAGGACAGCGTTATTATTAATTCTCTTTCTTTGAGAGTTGACATGAAATT CTGGTTTAGCCCAGTGTCAGTGTCTTTTGAGATGAGGCAGTGTTGTTGCCTTAGACAGCCTTTACTTTGCTTT TTTTATTCTTTAATAGCTACCTAAGTTGTAAAGAAAAACTACAACTACTACTGAAAAACTCATGACATTCCCA TCAGCCTCAGCTGTAGTTTGTGTTTTATGCTAATTAGCAGATGTTAGCGTGCCAACACGAACATGGCAAACAA TATACCAGCTCAGCATCACCACGTTAGCGTAGCCATTGTGTTGTATGTTAGCATGTCGACTTTAGCATTGCTG TAGAACTCAATATCTTTATTTTAAGTCATTCTCGAAGTCCTGTTGTGATAGTTTCAGGCTTTGAAAAAAAAAA TAATTCTATCCATTTCAATCCCTTTTCATTGACAGTTGCTATCTGCTAACCAGACTGCGTCCTTTCCCCAGGG GTACGGCTGACTGGTTTGGGGTGAGCAAAGACAGCGACAGCACCCAGCGATGGAGGAGGAAGAGCCTGCAGCA CTGCAGCCATCTGTACGGGGGTCTCAAGGCGCAGGTGATGCGGGAGATGGAGCTACACAGCCAGGACAACCTC TCCCTGGCCAGCACTGAGACCCCTCCTCCCCTCTACCTCCCACCCCACCACCCCAATCACCACCACTATGGCA TGCAGAGGGTAGGCCAGCGCCCGGGGTCACGTTAGAGGGACGGAGGAAGAGATGGGACAGTTGTTCACCTTTT CCTCCGTCGCACTCTTTCACTCTCTTTCGTTATTTCACATTAGGTTTCCGGCGCTTGCTTTACCTCTTTGCTT TGCCATCTTGCCTAGGGTTCAATTGATGGCTTTCTGAAGACGCATGAAGCAAAACTAATACAAGAAACTCATA AGGCTGCTACACAGGCATGGAGTACTGTGCATTGTTCTGTATTTGCAGCCTTAACTTGATAAATACCATGTAA TGATAACTGGCTGCTCAAAATTGGATTCTGACACACTACTTTACTCTGTTGAGATTTGAAATGTTTATACATT CAAACAAATAAGCCTCTAAAACCTCTAAAAAGATTTCTTTCTCTATACATTTTGTTTTAACTCATAGCATGCT AGCATAGGCCTCTAAGAGCCAGTAGCCCACAAATGCACCCGTATATACAATCCTTGGGTTTGCTTTTTCTCAC TTGTTTTAACAATTTTTATCACAAACACAAAGATTAGAGATTGCATTAATTTTGATCATTATATAGTTATTGT TAATAAGTATGGTCTGAGAATGTTTCACACTAATGGTTTCAAAGGAGGTTTAGTCCTCCTCTGTCCTGTTGAT TGTTGCATGAAGCATGAAGTACATTTTATAGTGGACAAATAGCCACTATAAAAGCAGCTGCTACTGTAAAGCA CAAGTTAAAATTTGTTATTAGATATTAAAAATGTGCAAATAACATCTGTAAAATATCAGTGCTCTGCCACTAA AACCTACTGCACTTTGGTCTTTTTAAAAAGCTCTACTCTTCTTGGTACATCAGTCTAACCCTAGTTTAGATGT GTGTCGTGTGCATCCCTGCTTTCCCCTGTTCTGCCACTTAGTGTTGTATGTGGCTTGACAATTGAGTACCAAA AAAAAGCTGTGCTGAACCAACACATACTGTGTGTGTGTGTGTGTGTGTGTGTGGGTAGATTGTAGACCCCCTG GCCCGGGGTCGTGCCTTCCGCATGGTGGAAGAGGTAGACGGCTTCAGTGTCCCGCAAACTCCCATCACGCCCG GCACAGCCTCCCTCTGCTCCTTTTCCAGCTCCCGCTCCGCCCTCAATCGGTTGCCACAACGACGCAAACGGGA GTCTGTGGCGGTCATGAGTCTCAAAGCTGCAGCAGCATTAATGAAGGTGTTTGTTTATTTATTGTTTATACGG TGCATTTTATTTTGAATTTCTTTCATTCGCCAAATATTCTGTGCTTTGCAAAATCTGTGCTTTAAAGTTAAAA GCTACCTCCATGTCCTGGCAATGTAACTGCACATTAGGACCAGCTGTAAGTAGTAGCAGTAGCTCACTATCTT CAGTCTGAAACAGTCAGAGCTCACAATATATGAATGCTTTTATGATGTTTTGTAACAAATCATTTAAAGACCA GGAGTATAGTCAGGGATAGGTTTCCATGCACATGTGCACTTTAATGTCCATTATTTTAGGTTTTTGTGAAGAG ATTCCTAGAAAA 
$>$ MN Cluster

TAA $\bar{C}$ AAAAACTGCAGTTCATCAAATGGCCCCTTGAGGCTGTGTGCATCTCCATAACGCCCCATATTAAATGCT CAACGTTACAGCCTAGTACAAAAATAGTTTTGGTCTCTATAGTGAATTTTCCCATTTCCAACAACTGTACAGG AАTTTTAтTTTATATAACTCAACCATTTAAATGATATTATGGCCTAAAGTGAAGGACACAGACGCTTTGAGTG ACAGGTGGATGCTTGAGTGACAATGTGTCCTGTTTTCAGTAACCAGGCTTCATTCAACTCGGCTCAGCTCCAC CCATGTTTCATGTATTTGCACATTTATAGATTAACCAGGAGTCAAGGCTGTCAAGATGGTGGTGGCAAGTGCT GACCTCCCTGAGCTTCTCAATGGCCCTTCATAAACCTATGGGTTACGTCACAGAGTCTACATCCATTGTTAAT TATACAATCTATGGGCCTTTCATGTTTTTTGCAAGTTTTGTGGCCACCATAATTTCTCCTAGTTCACAGCCTG TCAGTCTGAAGTCGTCAAATGCCAATGCGAGTGATGATCAGCTGGTTATGATCTGTAACTTCATTGCTATATC TCACACACTGGTCCTTTTGAACTCCTTTTCATCCCTATGAGTCAGATGTTTAAGGGTTTTCCTGTATTCAGTC ATtAGgTTTGATTATCACACAGTCTTTAAAAATTCCGTTTCCAACATGTCATGCATCCCTCTCATTGTTTGT GCTGCTCTTGTATCACCTGTCACACTGATTGCCCTATATAACTGACAGGCCTCTGCAATGCTGACAAGCAGAA CAAGGCGGGCTACACAGCGATCATGCTGACAGCTCTGGCTGCCTTCCACTCTGACTGCGACCTTCAAACCGTC CTGCAGCTGCTGCGCACAGGGGATGTCAATGCCAAGGCCAGCCAGGTGCGCCCTCTACTGTTCATTAGATTTT ACTGCACCTCCACCCACAATTAAATCCATCCCAGAACTTCACTTACAGAGAGACTACATTTGCTTTTCAAAAC TCTGCCACTAATGTGCTAATCACACTGTAGAGTTTGTCAGCAGATGTATGATAAAGCATGTAAACAAATGCA TTTCTAAAAGATTTTAAATAATGTGTGTTTGTAGGCCGGTCAGACAGCGCTGATGCTAGCGGTGAGCCACGGT CGAGGGGACATGGTGCGGGCGCTGTTGTCCTGTGGGGCACAGGTCAACATCCGTGATGACGACGGCTCCACAG CACTTATGTGTGCCTGCGAACACGGTCATGTGGACATTGTGCGTCAGCTACTGTCTGTGCCGGGCTGTGATGC CACTCTCACCGATAATGTAAGCACATTTCCCTCCTTTCTTTCTCCGTCTCTCCTTTGTTTTTTTTCTGTCCAA ACCCATCATTGTGCCATCATCTTCTCATTTTTCACAGGATGGCAGCACTGCTCTGTCCATTGCCCTGGAGGCC AGCCAGAATGACATTGCTGTGCTTCTGTACGCTCACCTCAACTTTGCAAAGCCTCCTTCCCCTGTGAGTACTT TGACAAATGTGATGTTAAGCTGTATGGTATAAGCATAAATATATCTGAACTTGGGATTCTCCTTTAATTACCC ACATTAGCTGTATTTTTTCTACAACTTTATGTGTTCTAAACCAAGTCTACAGTTCAAATGCAATAAAAACAT CCCCTCTGTATAATCAGTCATCTGCAGTATACTCAGCTAAAGATGTAAGCTGTCTTTCAACTGTCTCTAGGTT TCACCAAAGTCTCCTCTCTTGGGTTCGTCTCCTCCTGCCGGAGAAACAAAATAAAGTCTGCTCCTTCTGCAGG TGACAGCAACTCTACAGCCAATTCACAGCAACTACTCATATACCAGTGTGATTTTTTTTTTTTCATTTGTGTA ATTAAGAGAAACAATGAGTTTGTGTGTGCGTTTTTTTTCACCCTCTATAAGTACATTATTACGTGCAGATTAC ATGACAGCTTGCACCCTTGTTACCCATTGCTTTTTTTTTTACACAGTGGTGGTTGAAAACATGGATTTATTGT GTGGTACAAACTGAACATCTAGTGGCTGTTTAGCCGTCAGTCCAGTCTTGCAATGATCCACTTGTCAGCCATG TGGCACTTAGAAGTCAAAACTGTTCACTAAATTACATTTACAGTTCATTATTATACACTTGTTGTTTTATTCC ATACAAATCTCGTATTAGTTGCTTTATAGAGTGATGTGTTTCATTGTAATACGTACAATAGCTCTTACAACG TAGCATCATTATGTGTGTGACCATCACAGCCATTTGGAATAACAAAAGCCACCCCTCTCATGGATAAGTGTCC TTGCATCAACTAGTTCATTGGTGCTAGAAAAATATAATACAGAAAACACCACTTCAACAGAATATCAATATTT ATACACAAGATTTATTTACTCTAGAGTATTTTTGATATTGATTTTTTAAATTCTGACTTAATTCAGGACCTCC AAgGATCAAGATTTTTTTATTTCCCAGGTAATGGTTAACTTGTTACACCTTTTTAACCAGAGGACAAAAATGT ATTTTATTTTTGACCCACATTTCCTAAAATAAACCCAAAGATCTCTCCTATCAGCTCTGTGACACTGTATA TTGTGGTGATAATATTAACACAGGCCTAGCATCAGTGTGAAGGAAGAAATAAGGTCAATATTACTTTCTGAAG GTTATGGTACTATGGTCTTTGTATTTTTTATACATGAAGGTATTTTGGATGGCTTTAGGGAAAGATTAGACTG TATTTGTGTTTGAATATTCTGTGCTTATATGATTTAACAAGACTTTGTTGAGAAATGACTAAAAGCACTGGT GAAGCCACCTCCACTGTGGTCATGTCAGTAAGATTTGTCAGGGAACCAGAAAGTGACTGATTGGGGTCTTTAA AGGACGTGCCATGTAAGATGTGAATATCAAAATAATATATATCAATATAAACTGGTAGTTAATATGCCGAAAA TCAAATTTCTTGAACTAAAGTGCATAGGCTATTGATGTCAAAATTTCTATTACTTTTTAAAGATTTTTTCACA GCTTTCATATTCACTTTTAAACAACTGAATTTTTATAAACTCTCAAGTTTTTATTTAAGTATCTATCTTTTTA TACATTTAGAATTTTAAGCTCACACCTACTTTATGTAATCGCACAGCTCTTTAGTTTATGTTTTATGAAGGT TTATGTAAAATTATGGATTGTGTTTCTCAATTGATTAATAAATGTGGACCACACTGTTTCTATTGTGTTTATT TAATACTACTTTGTTTGCATGTTGCATCAGTAATGTAACACAAGGTAACAGTTCCTAATAAAGTCATGTTGCT TGTAATAACAATGCGTAAATTAACATAGATCATGTTTTATATTTGTTTTTGTTTTTTTTACAGTATTGAAGTT AGTAAATCTGTCTTTCATATACTTGGTATACTTTGTTAGGAAAAGCCCACTAGCGGGAGTTCCAACTAAATAA AGTTCTAAAAAAGTTCTACGACATACAATTCCCTCTCAAAACAGGCTAAGCAAACAAAACTCCCACACAATTC TCCTGAAACAAAGTGGAAAATAATCATACAGTATGCAATTACTAATTGAATATCTAATACAATTGCATAGTAA ATTAATAACAACACTGTTGAATACTAAAATGGAAGAAACTATAAGGAATAGCTTTACATTGTCAATGCAAATG ACCTATATAGCTACTTCCTGTAGTGTATATACAAATACTGTCACAGCTGCTGCGCATGCTGTGAAGAAACCAC CTCTGCACAGTGAACTGTTGTTTCTACATGTCTCCCTTCAAATTGATGTGCCCTTGTTAAATTTCAACTCAGT GTGAGTCTTTACTGTGTTTTGCTGTCATTCGTGTTCCTGTCATGAGACAGGATGCAGCTAAAGAGGATATCCA GAAGTTGCTGAGCCATTTGGCTCAAGTCAGTGCCAGTAACAGAGGTGTTATAATGGGAAACTTCTTGAATAGA ATCTGGTCCACTGGCTCTGAAAAACATGGCAAATCCCACTGTGGTGCCAGAGAGGATCCCAATGAACTCTGCC ACCAGATACACAAAAAAAGGTGGCATTGATGGATGTATAAACACAAACCTGTCACTAAAACCAGAGTGACAAG 
AAgAgATACCCAAAAAGGGATCTGGTTTCTCTTCATGATTTTCCTTTCACAGTGTATAAAACGTGTGAAGCCA GAGAATTCCCAAATGTCATACGTCTTGCGGTCTGCCTACCTACCTACTTGCGTACCTGTTGTAAGTTGTTTTG ATTGACTTCTTTAACAACTTGCCAGCCCATTGCTGCACTGTGCTGACTGTTAACCTGTGTTTGCTGAAGTTCT ATCATGCCTCCACACTCATATTCAGATAAACAAATCCTTTTTCTCTTTCAGTGGAAAAGTTGCCATCTTCTTA AAAGACATTCACGTTTTTAATAGATCTTCAAAAAAACAACCTTGAGCTCCAACTGGAGCACCATGCAGGCCTA AATGGAACAAGGTAAGATGCAAGAGGCCTCAGCCACTGACCATGTATCAAAAGTGGCACAACAATTTCATGTC TCCTCACAAGTTGCAGCTCTTGCAAGAAAGTACAGTCGGTACATCCTGCAGGATTACTAGTCAATGAGTAACT CCTAATAGAATTTATAGGGTAGCCAATGATTAGTTAATGCTTACTTAATGAAGAATTTAGTGAGGAATGAAT CAGGAACAACTTGATGATTGATGAATTGTTGATATCTAGTTCCCTAATAATTTCTAATGGAATATTAGTAGTT CGTTAGCTACAGTCCTCCATTAGGAATTATTAGGGAACTACTTATTAATGATTCATTAATTATCCATAATAAT AATAATTATAATCCAGTGTTCTGATTCATTCCTCACTCTATCTTGATTGAGTAATCATTAACTAATCATTAG CTATAAAACATTATTAGGATTTACTTATTCACTAATCACTAGCAGTTAGGTTATATCTCATTTGTTCTCTGGT AАCTACTCTAAAGTTTGTGTCCCTTATTGTCTTCTCACTGTATGCAAATTGTACTCTTATCAGCAACAACCAG GCCTTGGACAACCGTATGCGTTCCTCCTTTGTCATAACACCAGCTGATTTTCTCAGACAGCTGCTTGCCTTTG GGAGGAGTCTGTAAGATAACACGCCAATGTAAGCAATGCTATACTGTGCTTATGTTTGAGATAATAGCATTTT AGGACACTTACCAGCCAATCCAAAATCAGCTGGATGTTTTTAATTCTACCAAGGCAAAAAAGGTCCACTCAGC TTTCACTTTTATTTACTTTTGAATATGTTGATTGAATTTCAACTTCCAGTATATTGAGTCAAGACAACATAT CAAACATAACGAAGTATACATTGCATCAAACATATATGAGTAAATGAAATAAAAATGTATTGGAAACTCAAAT ACGTAAACTAAAATACATTATTATACAACTATATCAGTGTGAGCAAATGTAATGGGTATATAAGTGAGTAGAG GTGGAGGTCAGAGGAACATCTACTGTTTAACTGCAAATTTATATTCAAAGTAAAAGAGAAAAGGCCTCCAAAC TTGGAAAAACCTCTTAGTTGCAGAGTATTCAAGTTCTTCCAGTTTTAAATGTCCCATAACATTTTCTATCCAT CGAGAATGCAATGGTGGCTTGTTAGATTTCAACTGTAACAATATTAGTCTTCGTTGTGGCTTCATTTTGATTC CAGTCCTGGGAACATTTTAGCAAGTTGGAGTCTGTGAATTATCTTAAATTGCACATAACACAGGTCTTGTGGA AAATCTACATGATATGTCGAATGGATCCATTCAATTGCAAACTGCCCAGCTTTTGAAATATCAGCTCCCCATT GCTCCCTGAGATGGTTCAAAGAAGGAGATGCTTCCCTTGGAATTGCACTGTACAATCTAGATATCAGACCATG CTGGAAGAGGATCCCAGTCTAAAAGTTCCTCAATCCAACAACAAACTGCTTTTTGACCAAATGCACTTGCAAA TACCTGAGGAAATGGGATTGTGGTCATATTCTCTTACACTATACACTGAAGGCCCATCCTAAGCCATGACTGG AAAGCAGAGTCATCTGGAAATAGCCCATTGGACACAATAGGTAAGAAAAGAAAGTGCCTGTTTATGTTTCAAG TTTAACCTTAACTGTTTGACAATGGGGTTGACTGTCTTTTGCTGGGTTTTTTTTGCTAAAGGTAGTGGTGCAC AGATAATTGATGGTTAGGACATGCTTAATTTTGGGTACACTCAGTTACCTGTCCATTTAAGTTTTGTTGTGTT ACAGTGTGTCTCAAAGAATTAAATGATCACTTCTCTTATATTAGCTTTAGTTTCTGCTCAAGATAGCAAGTGA AATGGTAGTCCCATTAGACCATTTCATTGTGAAAATGTGGCCTTATTTAGCTCTTAACTCATCCAAAAGCCTT AAAAAAGCCTAAAACAATTACTCTGCTCATTTGGTTATAAATTGACTGTCAATTTCCAGTTGTGTAAGGCAGC AGTTTATGACTTTAAACTAGATATCAGCTGAGCAACACACTAGAAAGATACCTTATCTATTTGTTATTTTGAG TGGATCCACCCTCACAGCTGTCAGCCACTGCGTAGCAGGTTAGATAAGCCTTAGACGTGTGCTATGTATTGGC AGGAAACACAAAAATATGAGTCGAAATTTAGTCATCCCTTTGGAAGGTTTTCAAATTATAATCACATCTTTTA ATATAATTGATTGAGAACCTATTCAAATCTGTTTGATATTTTGAAAAAGGAAAGAAGGAAAAAAGGAAA TTTGTTTTTATCTCGCACAAACTATGACCCATCACTTGGACCACCTCCCGCAGCCTATCTTGGAGGAGGTCC CGGGTGTGCCCATTGTCTGTTTCAAGCATAAAAAACCTCCCACGGTTTGGGCTCATCACTGCATTGAGCAACT CTCAATCAACAGACAAGATGACCAGTCTCACAGCTAAGGACAAGGACGCAGTCAGAACCTTCTGGGCTAAAGT GTCCGGGAAGGCAGAGGACATTGGCGCCGATGCTCTGGCCAGGTAAAGATAAGAACATACTGACTGATATAAA ATGCTCCAACTTGACTTGATTCATTTACATACCTACTCACTCATGTATACAATGGCGTGCACAGACTTTTTGA AGGGCAAGGGCAGAAAGAAAAAAAAGAGCACATATACCGCGTCCTCACCACTATAGAGGGCACTTAACACGTG TTTTGGCTCCCAAGAGGGCACTTTAGCACGTGTTTTTCAACAATTAGGCCACGAGGGGGGGCGAAGATAAGAA CATACTGACTGATATAAAATGCTCCAGCGTTAATTGATTCATTTATTTACCTACTCACCCATGTATAAATGTT TTTTATTTTATTTTTACCCAGGATGCTGGCTGTGTACCCGCAGACCAAGACTTACTTCGCCCACTGGAAGGA CCAGAGCCCCACCTCTCCCTCAGCCAGGAAGCACGGAATAACTGTGATGACTGGAGTTGCAGATGCTGTGACC AAAATCGACGACCTGAAAGGAGGTCTTCTGAGCCTCAGTGAGCTGCATGCCTTCACTGTGCGTGTGGACCCTG CTAACTTCAAGGTGCAGAGCTCAAGTTTTTAAAAGATAATATGATATGAAGCGTTCCTCTAACTGAATGTAGA TGATGCTGTTCTTCTTTTGGCCTATACATATGAAAATTGGAGTATGGACTGATCATTAAGTGTTGGACAGCTA AAAACTCAAATGTAACATCTGTGTGATTGTCTTCTTTTGACAGATTCTCTCCCACAACATCCTTGTGGTCATG GCCATCATGTTCCCCACTGACTTCACTCCTGAGGTCCATGTGGCTATGGACAAGTTCCTGGCTGCTCTGGCTC TGGCCCTGGCTGAGAAATACCGATAAACAGCAGGAGAAGATGACGGAGACTGCAGCATGAGCTTACATTGCAT GATGATCAATAAATGCATGAATGCAAAAATGCAAAGCAATTGTTTTTCTGGTTTGTTTGTGATGATTTGTGTC ATTTGAGAGTAGATCAAAAGTCTCAACTACAAGACAGAATTTTAAAATCCAGTCAAGTTTTACACTTAACATT ACAGCTGCAACAATTAGTTGATTAATTGATTAGTTTTGATAATGAATTATTGTTACTTTTTAGGTAAAAACAT TCTGTAATTCCAGCTTCTTCAATGTTCTCTGTTTTATGTCACTGTAAATTTAACATCTTAGTACTGTTTGTCT GACAAAAGACATTTGAACCCTGGGAAACTTTGATGGGCATTTTTCACTATTTTCTGACATTTGATAGTCTAAA 
TGATTCAGCAAGTAATTTACACACTAATTTGCCGAGTCATCGATAATAAAAAATAACCATTAGTTGTATTTGT AACATCTGTAGTGATAATTCTTAGAAAACAAAAAATATAAAATAGTTACACGTTGTCACTTTGTGCAAATCCT AААААTTСАAАTACAATGTTTTAGTGAAATCAACATTTTTCTTAGAACATTTGGATAAATTAACTTCCTATG TGGCATTAGTCCCTTGTTACATGAAATAGGCCCTATGTAATAACTCATGAGTGTCATGTGACAACTGTCAGCC TTGAAAAAAAAAACGTATTTGTGCTTCTTCTGCTTCTTCTTCTTCTTCTTCTTCTTCTTCTTCTTCTTCTTCT TATTATTATTATTACCAGTATTATTATTAGTAGTGTTATCAATATTTTTATTATTATACTGTTGTTGAATTAA GAATATAAAATATTGAACTTTCTGCATTCAATTGATAATAAAGCAGAAGACCACAAGGAATTAGCCTGAAACA GAAAAACTGGACTTTGGATGGTAAACCTGATAAATGGTGAACCTTTTCTTTTAAATTCTACTTTTTTTTTTTT TTTTTTTTTACCTGTTCAAGTGGTGATCCAAATACCACATCATGAACAATGTATTTGTGGAACTATTAATGTA ACCAACCTGACATATTTCCTCTATAATGATAATAACAATAATATAATAAGAACTTTGTTTATGTTACGTGAT GATGGTAGAATCTTTTTAAAGAAATGTTCACTGTTGCCACATTTCATCAGTGCTATAGTGTTGCTGTCTATA CTTCCTGTGTCTGGTCAGTCAGAATAATATTTTTTATCCCAAATGAACATTAATTTATGTTTACATGCAATTT AGGTATAATTACAGGAAAACAACTTGTTGAATTTGCTTATTTTTATTTGCTTTTGAGAAAAAAACAAAGAGCT TTTAAACAAATACAGCATGTGATAGTCTTCTTAGTGGTACTGCCTTCCCAGGGATGACACCACCACAGCCAGG AACTTCTGAAAAGCTGCCTGGACATCACCAGTGAAGTCTTTACCCAGCTTTGCAGCAACCACAATGGTCAGGC AGTCAGACAACAGCTGCAAGACAACAAGCATCAGAAAACATTAACATGAAATGCGTTTGTTGAATAATGGTTG AATATGGATAACAGTAGTTACAGTTCCACAACTTTACCTTGAAATTGTCAGGGTCCACGTGCAGTTTCTCGGA GTGCAGCACGCTCAGTTCTGCATATGTGGCCTTGATGTCGTCCATGTTCTTCACAGCCCGGTCCAGACCGTGG AGGATGGTTGTTCCGTGTTTTGCGACCAACGGATTCGCAATGATAGCAGCGGCGTTGTAGAGGTTTCCAAAGT TGCCGAAATACCTCTGAGTCCAGGGGTAAACGACCAGACACCTGAGGGAACATGCAACAAGATAAACGTTACA GGCTAAGATAATGAGATTAAATAGGTGTTCCAGGAATATGAACATGCGTGCATACAGTGCACAAATAATAATC CTTGATTATTAATGTAAAAAACAAAACAAACATCTGACCTGGAAAGAGCTGCAGGTCCCACGACCTCATACTC CATCTTGGAGAAAATGTCCTGGATGGTGGCGCGCTCGAAGTCTGACCATTCAACCATTTTGCCGGCTGTAGAT TTTCCTGTGCAGATCGGTAGATCCTGTGCTCGTCGGCTCCAGAACCAGCTTTTAAAGAAACCCTACACCCCTC CCAGCAACAAGCGATGCGATGGGCGGTGGGCTGGACGAGTTCTGTTATTGATGATCACAAGAGAGATAATAGA AGGGCGTACCTGCACCACTTGTCTTTCTGAAACATAGGAAATGTTTATTTTCTGACCGTAAAGTCTGATGCAA AGTAAAAGAAAAGGCCTCACACGTAAAAATATATATATATTGAAATGACAGGATCCAGATATCAAGTGTGTTT AAAAGGCAAATTATGTAAAAAGGGTTTTACAGTCTTTACTGAGACTTATTTTCCGTTTCTGTCAGTATCTTTT GGACACATGGGAGCAAATTCAAGTGTCCACAATTTGTTGGCAGGGAGCCACAGACGGATATATGCACATTGAT GACCAAGATCAAAGCGAACTGTCAGTGTGTTACCAAGAGAGGGATAACGGCACAATAACAGGTTTACCCGCAC AGCAAAAAACACCTGTGGACCTGAACCTCGCCCTGGAGGTCCCAGGATCAGCACCTTGGACAGCGATCATTAA AgTTCAGTGCGGAATGATCGCACATTAAAAAAAAGATAAAACATGTTGAATGACCACCTGAGCTGAGGCAAGA TGGAGCAAACAGCAAGTTGTTCTATTGATAGTCTGTTAATGTTATCTCTGCCGAACATGTACACCCTAGACTA TTTTTTTCTAGCTCTGCTTAGCTATCAGTACACCAACAGGTGGTTGTGATATAGACTGGGTTGGGTCAGATTA CTTTGTAATGTAGTCCTTGACTGAATACAAAATACATGGCAATTTTGTACTTGTTAGTAAATGAATTTGATTT TTAAAGAAAAACACTTTTGGATTACTATAATATAAAACATAATATTATACCGTATGCTAAAGATTTGCTTGTT GCATTTTGTGCATGCGTGACTTAAAACATTAGTAAACGTTAACCTAATTAACGTATCAGTGTACATATTAAA GGAAAGTAATAATAAATTCATTTGATTTTTTAAAAAATCTAATCTTAACAGTTTTGGATTACTTCAAAATAA AACATAATATTATACCTACCATATGCTAAAGAATTGACTCAGAAATGCATGTTACACATTTTTGAATTAATTT GGTGATATTACCAGGAATGGGAGGCTGTTTTTGAGGAACTGCTGCTGCTTGAACCTCCCAACTTGACTCAACA CCGTTCTTTTTTCTCCAAAGTTCGCCACTAAGTGGAGCACTAGCCTAAGAAACTCCGTATGTTAGAGGAGACG CACTTTTCATTAGTATATATAATAATACATTGATTATCATGAAAAAGTACTATAATTTCAAAATTGCTATATT TTAAATCCTTAATCAAAATATGTAATCAAGTAACCCTTGACAATGTAAAACTAATCTTTTTACACATGTAGCA TTAGTAAGGCTGCTTATGTTTTGTTATCTGATTACTTAATTTTGATGACATGTAATCAGGTACTACCCAACTC TGGATACTGAAACCCCATAAAGTCACATGTAGCTTACCTGATCCTGCGGGAAAATAAATAAAAGAAAACACTG TGGGCTACACATCTCTCTAACAGCTTAAAATCTTATAAAAAAACAAATGTTATGGTATTAATATGGTATAAAT ATGGCTGTGTGACTCAGTCTGAGGCAGCAAAATGAAAAATACATAGGTTCACTTAAAAACGAAAAACGGACTG TACTTTTTAGTGTAGCCCTCCTGCCTAATTTTAGTGTCCTATAAGGGTCCTATGTGAAAGCTGTCAGGTTTTT TAAATAATAACTGTATTATATGTGGTTTAAATCCCTTTGTCTGCATACAGGCCAGTTCCGACTTCAAGTGGCC ATAAGTCTCCATCTAAAATATATTTTAAAAGATTAAGTAGAAGCCTATCACACACCTTTATTTTATTTTATTT TTTATTTTTGTATTTTAACATGTATTTAACCACATGGGAGATGCAATTGGGATAGTGCAACTTTTAAATTGTG TTTTACAAAATATACACACATAAGTCCACGATTTAAAAATATTTAGGTTGAGTGATGAAATTCAAAGAAAGTC CAAAGTCACAATGAGAAGATTTTTGTTTCTATCTAATGGAGTCTCACTATCTTGGAGGAGGTCCCGGGTGTGG CCTTGCTCTGTTTCAAGAATAAAAACCTCCCACGGTTTGGGCTCATCACTGCATTGAGCAACTCTCAATAAAC AGACAAGATGACCAGTCTCACTGCTAAGGACAAGGACGCAGTCAGAACCTTCTGGGCTAAAGTGTCTGGAAAG GCAGAGGACATTGGCGCCGAAGCTCTGGCAAGGTAAAGATGAGAACATACTGACTGATATAAAATGCTCCAAC

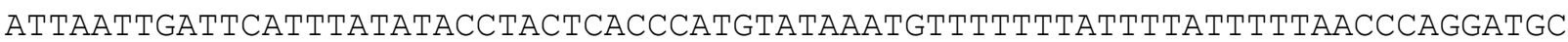
TGGCTGTGTACCCGCAGACCAAGACTTACTTCTCCCACTGGAAGGACCAGAGCCCCACCTCTCCCTCAGCCAG 
GAAGCACGGAATAACTGTGATGACTGGAGTTGCAGATGCTGTGTCCAAAATCGACGACCTGAAAGGAGGTCTT CTGAGCCTCAGTGAGCTGCATGCCTTCACTCTGCGTGTGGACCCTGCTAACTTCAAGGTGCAGAGCTCAGGTT TTTAAAAGATTATAGGATTTATATGAAGTTTTCCTTTAACTGAATGTAGATAATGCTGTTCCTCTTTTGGCCT ATACATGTTGAAATTGGAGTATGGACTGATCATTAAGTGTTGGACATCTAAAAACTCAAATGTAACATCTGTG TGACTGTCTTCTTTTGACAGATTCTCTCCCACAACATCCTTGTGGTCATGGCCATCATGTTCCCCACTGACTT CACTCCTGAGGTCCATGTGGCTATGGACAAGTTCCTGGCTGCTCTGGCTCTGGCCCTGGCTGAGAAATACCGA TAAACAGCAGGAGAAGATGACGAAGACTGCAGCATGAGCTTACATTGCATGATGATCAATAAATGCATGAATG CAAAAATGCAAAGCAATTGTTTTTCATGGTTTGTTTGTGATGATTTGTGTCGTGAGAGTAGATCAAGTAGAAT CAGAATAAAAAAATAATCCAGTCAAAGTTTTGATAATGAATTATTACTATTTGATGTAGCTATCTTATTTTAC TCTAGGAGTGTAGGCATTTTTCACATGTTTCATTATTATGTTAAGAATAGGCCTATAAAATATTAAACATATA TATTAAATTTTACACTTGGCCATTAAGCAGAACATCAATGAATTAACAACATGAAAAACTGAATTTGATTTAG TAAACCAGATATTTTATCAACTGCTTCCTTTAAATTCTTCTTTTCTTCTATTCAAGTGTTGAACCAAAAGCTA CATTATGAACATTTAAAGGCAACAATATATTCGTGGAACTACAGATAAGAAACCAGCCCAGTGTTTCCTCTGT AATGATAACAACAAAAACAGCAACAAGTATTTCACTCAGTAGAGTGCAGAAACCTCAGCAACAGATGGTGAAG CACCGACTGAAATGTCACCACAATTGCCAGATGTAACCATCGGGGATTGGCAAAGGTTTCTATTGTTTTTGTA GTATACGTTTTCTTATAAATATGTAGGCCTATGCAAACTCTTTTTGTAATAGTAATCGTGTAGTCTCCGTTCC CCACCATTTAAACATTATTTATTTAGAACATTTTGTTCACACATCACAGAAAATAGAAGGGCAGTATTTAACT ATGCAAAAGAAACAGGTGTAATATCCCGAATAAGACTTCCTCATAAAGTCATGATGTTACATACTTCGGTACA GAAGGTGGCGGCATGCACCCGTTGGTTTGTAGTCCGCCAGTAGAAGAAAAACAAGGGTCCCTCGCAGACAAGA TGGCGGCAAAAACAGGGGATTTTTAGGGACAGAGCCAGCGACCGCTGGAGGTCCCTATTGTTTTTGCTCGATT TCTTCTTCTTACCTATTATTTTTAGCCCCCATTGACTGCATTTTTGCCCCCCTGAACATATACGAAAAGTCAA TTTTATTTGGCAAAGTTGTCAGGCCTGGTGAAAATGGGGAAATTCTATGCGTCGCTTTCGGGTTGGCCACTAG GTGGCGCCTCTACCATAAAAAGTGCGTTTAGGCTAATAACTTCTGCATAGTTTGTCGCACATTCATAAACCTT GTATCCACGCGTTCGGTGAATGGAGCTGAATCTCCTCATATAGGCCCCGCCCACTTCCGCCTATAATTTTTAT TCGTTAGGGCGCGAAATGTCCTTAAACTACTTTTTCGTGGTCCTCCTACACGCTTTTTCTGATTCGCATGAAA TTTTTCACCTAGCACCTGGGGACCACCCTGGCAAAAAGAGTAATTTGAACATTTTTGAAATTTCAAAAAATAA GAACGCTATTAAATTCCAACTTCCTGTAGATTTTTCGTCAAAAAAGGAAGAGGACAATTTCTCCACATTGCTA TGTCCGATTGACACCAAACTTGGGTCCATAGTTCCACATGTCTTCCTTAAGCATCATGCACAATTTCAGGCCA TCGCCACTAGGTGGCGCTCTTTAAAATGTAGAAGTTTATATCTCTACAACGGTATGTGTGATTAAGATGAAAC TTAATACGATGCTTCCCCATGGCTCCCTGAAGCTCGCTAAAAAATGTGTCAACAATCCGCCACTAGGTGGCAC TCTGGTGGCATGGTAAATCACTGTGCGGAGACCGTAATAGTTATGAATATTAAATTCACAGGGCACGTGTAGA GAGGGGCCTGAAGCTCACATACCAAAAATCGTGTTTGTCGTGTGTGTAGCGCCCCCTACAGGATAAAAAAACA GCAGCCCCGGCGCCACGTTTCACCGATATGTACGAAACTCGGTAGGCATGTGTAACATGCCGGGACGTACAAA AAAGCCTCTTGGACCCATACCCGAAACCCCACAGGAAGTCGGCCATTTTGATTTAAAGTTTAACTTTCGGCGA ACTTTTCGCGTTGTATTTGAACGAACTCCTCCTAGGGAATTTATCAGATCTGCTTCAAAATTGGTCAGTACAG TCATAAAATGGTGCTGATTAAAAGTTGTGCTATTTGAGACTGTACGTTGAATGGGGTGTCTGTGGCGTGAGGT CAAAGTTGGCCACTTTGAATTCTGTCGTCATACAAACTGATAGAAAAGTTGAGCTGTGACTGTACATTAAACC GCGTGCCAGTGACAACTTGTCCATTTCAGCAGATTCACCATAAAATAGTACTCATAACTTCAGTGTGCAATGT CCAATCTCCAATAAACTTCATATATTCTGAAACAGTTTTGCCCTGAACACGTCCTCATAACTTCAGCGTACTT GGTCCAATCTCAATGAAACTTCACGTCTTTGATAACACACTCGCCTTGAACATGTCTACATGACAACATTCAG ACATAGTGATAGCGCCACCTACTGCCAACAGGAAGTAAGCGCTGCATGACGATTTTGAGCTGAGTTACGTGAA ATGACCGTGCCAGTGACGACTTGTCGAATTCAGCTGATTCGCCATAAAACAGCACTCATAACTTAAGTGTGCA ATGTCCAATCTCCAATAAACTTCATATATTCTGTAACAGTCTCACCCTGAACACGTCCTCATAACTTCAGCGT ACATGGTTCAATCTCAATGAAACTTCACGTGTGTTATAACAGTCCCGCCCTGAACTTTTCTATATGACAATAT TAAGACAAAGTGATAGCGCCACCTACTGGCAACAGGAAGTAAGCACTGTGTGGCAGTTTTGAGCTGATTTACA TGAATTTTCATGTCGTGGTCCGCACGTGTAACGACGGCACGCGACGCGGCCGCAGCACACCCCGAGTGGCGTG GAGTGCGAGGTCCCGCTCAACGCTGCTTGCAGCTTTAATTCTTCTTTTTTTATGTGCTATTTTAGTGAATCAT AACAGATCGGGTATGGGATTAATCTGCATTTGCTCCGGTTAAAGATGGCGAAAACGTTTCTGTGGCTGTAAGT TTTTAAACCAAGACCAAATGCCAAAAATGTGGCCTTCACACCTTGTTTTGAGCATAGCAGTTGTTGATCACTA GCGACCCCTGCTGGCCGGATAAGAGGAGGGAGGAGTCAGCAGTCGGCATAAAACAGAGCAGTCAATAAAATAG GGTTTTCAAGTTTAAAAAAATAAGAACTATGAATTGACTTACATCAACCAGAGAGATCATTGAGCATACAGTC ATGAATGTGCACAAAAAACACACTATGTTGCTATTTTGGTAAAGTAATAAGAGTAATTGGTCCAGTAGTATGC AAAATGACCCAGGTCAGAAAATATTATTTAGTGTATTAGAAATGATCGATGTAGGCTATACAAAAGTACACTT GTGTACAATTTACACTTACAAGTTTCACATAATTATAGTGCTAATTTAAGTCAGTCTGGCTGACTGCTGTTGA ATGGACGTCAAGACATTACTGATGGTGCAGATTTGTCTTTCCCAGACGTCACCCATATGACTTGAAGCTGGAG TGTTCACGATGAATTCATATCCCAGTTCTTGCTTCAATAACTCATGCAAAGTTTGTCCCCTTGATCACATCTT AACTTGTTAAGAATGGTCCGAAACTATGAGAAGCCGCCTAGGCTAAAACTGAATGCTTCAGTGGCACATACAT ATATACTGGTTGTAAGCACTTGCACATACATGCATACTCTCATGCATATGCATACATACATGCATGCATACAT 
ATACAGACATAAACACACACGCATGCATAATGTGAGAATTTAAACTGGGAGAATGTGTGTGCTTGAGTTTGTA TACTGTAAGCCACCATACTTTAACTGGAAGTTGCTACCATAACCAGCGCTTGGTGACAAGCTCAGCTCAATGA GCGGTAGTATGTGGTAGTGGGTGAGTTAATGTTTCTGCTTCATGAAACCGCCAAACCTTGTCACATTCAATTT AGAGTTCTTGAAGGCATTACTCCATTTGTTCTCTTTATAATATCACACTAGCCTTGGTCCAATTAATAGCTTT GAGGAAGA GTGCAAATCCATATTTACAAATGAATCAACATATTACAGTAGGTACTAGAGTAAGTAAAGGAAAA AGTAGTAGCAGTGATATTAGTTTCACTAGCTATTTTGCTGCACAGTTAATCCAAATATTATCGAAATGGCAGT ATGgCTAGGTGCAAATCGCAAATTTGTGACAAAATAGCATTATAATGAAGTACTGTAGTGCAGCAAAGATTCC CTGGCCTACAAAGCTTGTTATTCAGATTTAAGAAAACATGTTCGTTTGCTACACACCCCAAACAGTTTCACAT CACCATGATATAGCACTTATGATGATTCATATTATGATTCCGATAATATTTCAATTAATTAATTGCAAATATT СтTCTGCAтAстCCCACACTTATATTATACTTAATGCAGTACAATTGAATTGTAATGCCATTTTAAGTATATT CCCAACTCAGTGGTGATATACAATTGTACTGCAAGTGTGTTTCACATGCTCAAAAATCATTATTTTCCCTGGT GTACTTCAGTACACTGAATGAGTACTGAGTCCCAATTAACATACTATTTTCATTTAAAGTATACATCAAGGCG ACAGAAAAGTATACACATAATAGAAGTGTACTTAGTACATTCTTTAACAAGTATATTTAAAGTATAATATTTT TAAGGGTAATTATTAATGCAAAAAAACATCTGACCTGGAAAAGTCTGTACATTCAACCATTTTGCCAGCTGTA GGTTTTCCTGTGCTGGTCAGTAGATCCTGTGCTCACTGGTTCCAGATCCAACTTTTAAAGAAACTCTACTCCC CTCCCACAAGTAAGCGATTGGATGGGCGGTTCTGTGTTATGGATGATCACAAGAATGATAATAGAAGGGCGTA TCTTTAACGCTTGTCTGTCTTAGATGATAATTCATATTAAAATAATCACATCAAGTGTGTTTAAGAAACGTTT TTGTAAAAACGGACTAAAAACTTTACTCAGGCTTATTTTCCGTTTGTGTCAGTATCAGAGAACAGAGAGCCAC AGACTGATATATGCACATTGATCCAAGACCAAAGCAAAATGCCAGTTTGTCATGCAAAGACAGGAAACGTAAC AATAACAGGTTTACACACACAGCAAAAACCAAACATGAACCTGAATGCTGCCCATATCCTCCCACATCACTGG AGGTCCCAGGATCAGAACCTTGGACAGCAATGTTTGTATTTCAGGGCAGAATGATGAATGATTGCACACTAAG CTGAGGTGCAGGATGGTGCAAACTGCAGGTTGTTCTATTGATAGTCTGCAAATGTTATCTCTGTAGAGCATGT ATACGCCGAGGCTAATTTTAGCTCTGCTTAGTTATCAGTACACCAACAAGTTGAGATGGAGACAAGGTTGGGT CAGA GTACTTTGAAATGTAATCAGTGACAGAATTCAAATGACAATTTTAGAATTATTAATGTAATCTATTGGA TTGCACAAAAATACCACCTAAAATTTGAATTACTTCAAAATCAAACAATGGACATTTTTGGACACTTTTTGAG GGACTGCTGCTACTTGAACCTTCCAACTTGACTCAACACTGTTCTTTTTTCTTCCATCACTAAATGTCAGAGC AGATGCATTTTGCTCTATTGAAATAATTAATAGAGCATTGATTATCATGAAATATGTCATATTTATATAGTC

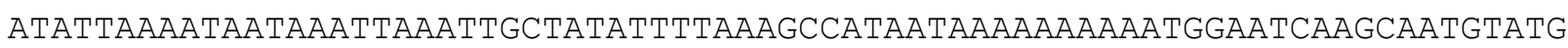
ACAATGTAACTATAATCTATTTACATCTTTTTTTCAAATGTAATCTGGGGTGTTCTTATAGGCTAATCAGTAG CTACCCAACTTACAGAAGCCCCATAAAGTCGCATTTACTAGATCCTGCTGGAAAATGAATAAAAGAATAAATT AAAGCTCACTCAGCTTAAAATCTTGCTATAAGTCTATGCTATTTTCAGTGGCTTAAAACATTAATTTCAACAT TGACCTAATTAATGTAGAACATAAACCTTTCAGCAGCCAGCGTTTGTCAGTGGAATTTTTGGATTGTATTTTC CAAAATATCCACATAATTTGAAAATATTGAGGTTGAGCGATGAAATTCAAAGAAAGTGCAATGAGAAGATGAG AAGATTTTTGTTCTTGAGTCTCACTATCTTGGAGGAGGTCCCGGGTGTGGCCTTGCTCTGTTTCAAGCATAA AAACCTCCCATGGTTTGGTCTCATCACGGCATTGAGCAACTCTCAATCAACAGACAAGATGACCAGTCTCACT GCTAAGGACAAGGACGCAGTCAGAACCTTCTGGGCTAAAGTGTCCGGAAAGGCAGAGGACATTGGCGCCGATG CTCTGGCCAGGTAAAGATAAGAACATACTGACTGATATAAAATGCTCCAACATGAATTGATTCATTTACATAC CTACTCACTCATGTATACAGTGGCGTGCACAGACTTTTTGAAGGGCAGGGGTGGAAAGAAAAAAAAGGGCACA TATACCGTGTCCTCACCACTATAGAGGGCACTTAACACGTGTTTTGGCTCCCAAGGGGGCACTTTAGCACGCG TTTTTCAACAATTGGACCACGAGGGGGGGTGTAGATAAGAACATTCGGACTGATATAAAATGCTCCAACATTA ATTGATTCATTTATATACCTACTCACCCATGTATAAATGTTTTTTATTTTATTTTTTACCCAGGATGCTGGCT GTGTACCCGCAGACCAAGACTTACTTCGCCCACTGGAAGGACCAGAGCCCCACCTCTCCCTCAGCCAGGAAGC ACGGAATAACTGTGATGACTGGAGTTGCAGATGCTGTGACCAAAATCGACGACCTGAAAGGAGGTCTTCTGAG CCTCAGTGAGCTGCATGCCTTCACTCTGCGTGTGGACCCTGCTAACTTCAAGGTGCAGAGCTCAAGTTTTTAA AAgATAATATGATATGAAGCGTTCCTCTAACTGAATGTAGATAATGCTGTTCTTCTTTTGGCCTATACATATG AAAATTGGAGTATGGACTGATCATTAAGTGTTGGACATCTAAAAACTCAAATGTAACATCTGTGTGACTGTCT TCTTTTGACAGATTCTCTCCCACAACATCCTTGTGGTCATGGCTATCATGTTCCCCACTGACTTCACTCCTGA GGTCCATGTGGCTATGGACAAGTTCCTGGCTGCTCTGGCTCTGGCCCTGGCTGAGAAATACAGATAAACAGCA GGAGAAGATGACGGAGACTGCAGCATGAGCTTACATTGCATGATGATCAATAAATGCATGAATGCAAAAATGC AAAGCAATTGTTTTCTGGTTTGTTTGTGATGATTTGTTCATTTGAGAGTAGATCAAAAGTCTCAACTACAA GACAGAATTTTAAAAAATCCAGTCAAGTTTTACACTTAACATTACAGCTGCAACAATTAGTTGATTAATTGAT TAGTTTTGATAAGAATTATTGTACTTTTAGGTAAAAACATTCTGTAATTCCATCTTCTTCAATGTTCTCT GTTTTATGTCACTATAAATTTAATATCTTAGTACTGTTTGTCTGACAAAAGACATTTGAACCCTGGGAAACTT TGATGGGCATTTTTCACTATTTTCTGACATTTGATAAGTGATTCATCAAGTAATTTACACACTAATTTGCCGA GTCATCGATAATAAAAATAACCATTAGTTGCATTTGTAACATCTGTAGTGATAATTTTTAGAAAACAAAAAAT ATAAAATAGTTACACGTTATCACTTTGTGGAAATCCTAAAAATTTCAAATACAATGTTTTAGCGAAATCAACA TTTTTCTTAGAACATTTGGATAAATTAACTTCCTATGTGGCATTAGTCCCTTGTTACATGAAATAGGCCCTAT GTAACAACTCATGAGTGTCATGTGACAACTGTCAGCCTTGAAAAAAAAAAACATATTTGTGCTTCTTCTTCTT 


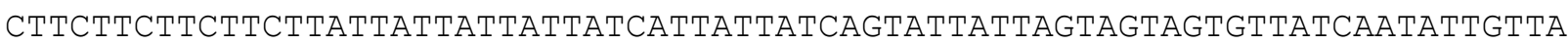
TTAтTATACTGTTGTTGAATAAGAATATAAAATATTAAACTTTCTGCATTCAATTGATAATAAAGCAGAAGA CCACAAGGAATTAGCCTGAAACAGAAAAACTGCACTTTGGATGGTAAACCTGATAAATTGTGAACCTTTTCTT

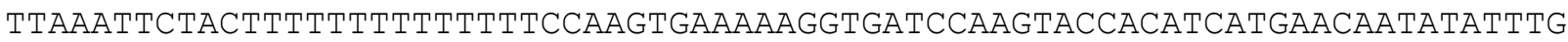
TGGAACTATTAATGTAACCAACCTGACATATTTTCCTCTATAATGATAATAACAATAATATAATAAGACTTTG TTTATGTTACGAGATGATGGTAGTATCTTTTTTAAAGAAATGTTCACTGTTGCCACATTTCATCAGTGCTATA GTGTTTCTGTCTATACTTCCTGTGTCTGGTCAGTCAAAATAATATTTTTTATCCCAAATGGACATTAATTTAT GTTTACATGCAATTTAAGTATAATTACAGGAAAACAACTTGTTGAATTTGCTGATTTTTTATTTGCTTTTGAG AAAAAAAACAAAGAGCTTTTAAACAAATAAACAGCATGTGATAGTCTTCTTAGTGGTACTGCATTCCCAGGGA GGACACCACCACAGCCAGGAACTTCTGAAAAGCTGCCTGGACATCACCAGTGAAGGCTTTACCCAGCTTTGCA GCAACCACAATGGTCAGGCAGTCAGACAACAGCTGCAAGACAACAAGCATCAGAAAACATTAACATGAAATGC GTTTGTTGAATAATGGTTGAATATGGATAACAGTAGTTACAGTTCCACAACTTTACCTTGAAATTGTCAGGGT CCACGTGCAGTTTCTCGGAGTGCAGCACGCTCAGTTCTGCATATGTGGCCTTGATGTCGTCCATGTTCTTCAC AGCCCGGTCCAGACCGTGGAGGATGGTTGTTCCGTGTTTTGCGACCAACGGATTCGCAATGATAGCAGCGGCG TTGTAGAGGTTTCCAAAGTTGCCGAAATACCTCTGAGTCCAGGGGTAAACGACCAGACACCTGAGGGAACATG CAAAAAGATAAACGTTACAGGCTAAAATAATGAGATTAAATAGGTGTTCCAGGAATATGAACATGCGTGCATA CAGTGCACAAATAATAATCCTTGATTATTAATGTAAAAAAACAAAACAAACATCTGACCTGGAAAGAGCTGCA GGTCCAACGACCTCATACTCCATCTTAGAGAAGATGTCCTGGATGGTGGCGCGCTCGAAGTCTGACCATTCAA CCATTTTGCCGTCTGTAGATTTTCCTGTTCAGGTCAGTAGATCCTGTGCTCGTCGGCTCCAGAACCAGCTTTA AAGAAACCCTACACCCCTTCGAGAAACAAACGATTGGGTGGGCCGGAAAAACGATGTGGGATTTGCACCAATG ATAATGGTTATCTGCGCCTCTCATATTGTTTCTGAGACAGAAACCACTTGTCTTTCTGAAACATATGAAATGT TTAтTTTCTGACCGTAAAGTCTGATGCAAAgTAAAAGAAAAGGCCTCACACGTAAAAAAAAAATATATATTAA AATGACAGGATCCAGACATCAAGTGTGTTTAAAAGGCAATTATGTAAAAAGGGTTTTACAGTCTTATTTTCCG TTTCTGTCAGTATCTTTTGGTCACATGGGCGCAAATTCAAGTGTTCACAATTTGTTGGCAGGGAAAACAGGTT TACCCGCACAGCAAAAACCAACTGTGGACCTGAACCTCGCCCTGGAGGTCCCAGGATCAGCACCTTGGACAGC GATCATTAAAGTTCAGTGCGGAATGATCGCACATTAAAAAAAGATAAAACATATTGAATGACCACCTGAGCAC AGGCAAGATGGAGCAAACACCAAGTTGTTCTATTTATAGTCTGTTAATGTTATCTCTGCCGAACATGTACACC CTAGGCTATTTTTTTCTAGCTCTGCTTAGCTATCAGTACACCAACAGGTGGTTGTGATATAGACTGGGTTGGG TCAGATTACTTTGTAATGTAGTCCTTGACTGAATACAAAATACATGGCAATTTTGTACTTGTTAGTGAATGAA TTTGATTTAAAAAAAAAAAAATCGGTAACACTTTATATTAAGGTACACCAATTCACCATTAATTAGTTGTTTA TTAGCATGTAAATTAGTAACATATTGGCTCTTAAATAGTCATTATTAATACTTATTAATGCCTTATTCTGCAT GgCCTTATTATACAACCAGTAAgCCATTAACTAAGAGTTTTCCCTCAATAACCTCAGAATTATTGCTTATTAG TAGTAAGTTAAGAAGTTGTTGTATATGAATTACCATCTCAATATGCTAATATGTCCTAATAACTCTATATTAA GTCTTTGTGACTTAGAATATGTTCCCCATACTAAAGTGACATCTTGATCATTACTAATTCACTAATAGTTGAA САСTTATTAАССАAСАCATGTTCCCTAATCTAAAGTTGCCTCCTTTTCACACTTAGTTAATATATTATGGCAC ATAACTACTGCAATGAACAAGTGATGGGTTACACCTTTGAAAAAAATGGATCTATCTATAGGTTCCCTCTCAT TCAGTGGTAGAATAACAAAGACAGTACAAGTACAAATACCTCATTTATTAGACCACTGGTTCAGCTGGTTCAG GTTTTCCCAAGGCTGACAACAAATACTGTGTGAACTTGCTGTGTAGTTTAACTTACAGGTAATGGGGTCTGCC TGAAAATTCAAACACTGACAAGTATCAGTGTATATAAACATGTCATACAATATGGTATCAAATGTATAAGCAG GTCATACTGTATGATAATACAGGTACTCTATTGTTGAAAGTCTGTATGATCCTTTAACCAAAATCTGCCTGCT TACAAAAAACAACAATATTAGGTACAAAAATACAAAATATTGCACAATCTAAAAAATATAAAACCTGCACAGC TCAACATGAACTTGCTCTTTATCAGATACACTACACTTGTCAACGCTTAGAACAAATGTACTGAACTCAACAA ACATTGCACTTTCACAGCCTAAACTTGCTCTATAATTACTGTATCAGGTCTTAATATTTCATCAACAAGGGCA GGAGTTGGCATTTGGCCTTCATGTCCTCTATAATGCTCTTCTTCTCAGGGGTAATGTGGGTTGCACATCTCTT AGCAAATGACTGCAGGGTCTCAGTTGCAGGATCATACTTCAGAGTCTCGAAGGTTTCAGGATCCACAATGTTG AGCTCTGCAATTGCAGCAGTCATCTTGATGGTTCCCCGGTCCACGCCATTGATTCTGAAGGCTTCAGTCATGG TGCGGACTTTGCTTAAGCTTTTCAAGACTTTTTTTTATATCTGCTGATAACCTCAGCTGGCCCTTTCACTGAA AAATATATAAAACAAAAACAAAAGAACACGAGAAATGAGCACAAACAACGGCTTAAGGTCAGCTGAAATTGCT CAAACTAGTCACCTCTTTTTTGATAAAAGTATGAAGCATATTCTAAGAATGGGACAATCTGCTCATCTCCTGA TATGATTCCATGCACAATATGTGGTTCAGAATTCAATACAAAAATGATACTCTGTAAGTTTAGTTGGTAAGTC TTACGGTTCAGAATTATTTTTACTTCTGAGAGACAAATCTTTCTAGCAATACATTTGATGTTGAATAATAATT AGTGTGAATGCTGCAAAATAAAATGGAGCTTTATTCTTGATGAAAGCCACATTTTTGACTTTAGACATTTAAT TTTTGCCTCATAATTACTTCAGAACATATTTTTAAAAGCTCTTCCAACGCTTTTCTTAATAATTACTTTTCAT GACCACTTGTTGCCAACAGGGCAGGTGGTTCAAGTTATGCCATTCTTACCTCTCACATGAGCTTTTCCAGGCA CAGCCGCAGTTGCAGATGCAGGTGGCTGTGTCCGACGTGTCTTTCGTCTTTGGATGACAACAACACTGTCCGA GGACTCACTGATGTCTGGAGAAGATGGGGGAATGACGTCATCTTCCTCCATATTGCCATCATCATCCTCATCA TCGTCCTCCAAAGAAAATACCATTTGGCTGAGCTTTGATCAAATAGAGGATAGATGAGACAAAGATCAGATTG TTATGAAGATATATCACACCTTTCATTACAATTACATTATATTTTGCAGATACAAGTTGCTCCCAAATCACTG 
ACATGATTTCTTCTTTCCCATGATTTGCTCACGCAAAAATTGCGCTCTTGCTCAAGATCCTTTATTATTTTTT CTTGCCATTGGATCCTGGCTTCTAGGAACTCCACTTTGGAGATTGATCCTTAAAAACAAAAATGAGAGCAGGA TACAAAAAAAGATTATGATTTGACAATATTGAGTGAGTGAGAGCGAGAGCAAGAGAGAGAGAATGACATGCGT GCAACAGAGGTAGTGGACCAGATATGGTGTGTGCCTTAACTTGATTTGTAAGCACATGTATACTGACAAAATC TGCCAACAACTTTAATCCCAGGCAGAAAAATCACTAGCAAGAAATGAAACTGTAATTTTAGACAAAAAAGATA CACTGCTAGAAAATGAGAAATCATTACCTGATCCAATGCTGTTTATACTGCTGCAACTTGGAGCAGAGCTTCC TTTATCTTCAGGACCTACAGAATGGCATAGGTACAACAAAAAAAACATATGAATAGAGGACTTTAAATGTTTA AACACATTTGGAAAAGCTAATTATTTAAGTATTAAAGTTCCTTGAAAGTTGAAAGAGAAATATTTACCATCCA GATCATTTTTGGTCACCTCTGCCATCGAGTCATCTTTGGGGACTTTTAGGGACAATTTCTTCTTGGCCTTAGG CTGTAAAAATAAAAAAATTCATGTCACTATAACCGGCAACATGTAACTTAAATAATCCACACAAGATTCAAAA GCTCTTCTTGTTCTAATGACTGCATAGTGCATAATTTATTTTTAATTCCAAAATTTGGTTCATACTAAAGCAT TACATGCACCTACATTCACATTTTATGTTAATGTTAATGTTTCTTTCCTACTATTCACCCAGCTCACCACTCG GCTAGCTTAACATTACATTAGCTAATTAGCCTCTACACTGCAGATAAATAGGAGGGTTTACTACTCTTTAACT ATGTAAACGGCCGGGGATGTGCTTACAAAACTCAGGGGGAGTTTCAATTAACAGGCTAGCGCTTAACTTAAAT TTCCCTCTTCACTCTCCAAGCACTGAACGAAACGGCGTCTTTGTGCTGTCTTTGTTTTTCTACCACTGAATGA CAGGGAACCTATAGATAGATCCATTTTTTTCAAAGGTGTAACCCATCACTTGTTCATTGCAGTAGTTATATGC CATAATATATTAACTAAGTGTGAAAAGGAGGCAACTTTAGATTAGGGAACATGTGTTGGTTAATAAGTGTTCA ACTATTAGTGAATTAGTAATGATCAAGATGTCACTTTAGTATGGGGAACATATTCTAAGTAACAAAGACTTAA TTTAGAGTTATTTGGACACTATTAACACTTGTAGTTTTGTGTTTTGTTATGTAAGAACAGACCATATTCATTA AGTGTTATTAAGGGAGAATAACTATTCTTGTGATACTACCACCTTAGTCCATACTAAGCAAAGCATATTGAGA TCGTAATTCATATACCACAACCTCTTAACTTACTACTGATAAGCAATAATTCTGAGGTTATTGAGGGAAAACT CTTAGTTAATGGCTTACTGGTTGTATAATAAGGCCATGCAGAATAAGGCATTAATAAGTACTTAATAATGACT ATTTAAAAGCCAATATGTTACTGTGCTGTTCCTCTTTCGGCCTATACATGTTGAAATTGGAGTATTGGCTGAT CATTAAGTGTTGGACATCTAAAAACTCAAATGTAACATCTGTGTGATTGTCTTCCTTTGACAGATTCTCTCCC ACAACATTCTTGTGGTCATGGCCATCATGTTCCCCACTAACTTCACTCCTGAGGTCCATGTGGCTATGGACAA GTTCCTGGCTTCTCTGGCTCTGACTCTGGCAGAGAAATATCAATAAACAGCAGGAGAAGATGATGGCGACTGC AGCATGAGCTTACATTGCATGATGATTAATAAATGCATGAATGCAAAAATACAAAGCAATTGTTTTTCTGGTT TGTTTTTTGTTGTGTCATTTGAGAGTAGATCAAAAGTCTCAACTACAAGAAAAATTTGAATTAAATAAAAATC CAGTCAAGTTTTACACTCGATTAATTGATTAGTCAGTGACATAAAATGAATGTAAACTGTTTTAATAATTATT ATAGTGTTTTTGTTGTTGCAAAAACTGTTTTATACCATTCTAAAGTGGATATCTTGTTTGTCTGACAAAACAA AACACGAAATTTGAATTCTGGGAAACAACATTTTCTAGACTAAAGAAGTTATTTCCAAAATAATTAGAAGATT CATTTAAAAAATGAATTGTTAGTTGCAACCATACAGTTAAACTTATAGGGACAGCAGTTGTAACATTTGTTAC ATATACGTGTATCCCAGTCTTTTGGAAAACAAACTAGCAATAAATATAAACCTTATTATTTGTCAACTTTTTG СTTTAAATCCTACTTTTCTTTTATCTAATCCTGCGAACATCATGAACATCATGAACATTTAAAGGCAACAATA TATTTGTGGAACTATTCTACAGACACACAACCTGCCCTTTGTTATATCTTCAGTGATAACAACAATAATGTCA AGGATTATATAATCAAGACATTGTTTTTTTATTTTTATTATCTAATTTATTTCAATCATGTTTCATCTTTGTG TTATTTAGCTGGAGTAGTTTTTTTTAAAGACAGTTTCTTGTTGCCACATTTCATCAGTGCTTTTTTTACCTGT TCAAGTGGTGATCCAAGTACCACATCATGAACAATATATTTGTGGAACTATTAATATAACCAACCTGACATAT TTTCCTCTATAATGATAATAACAATAATATAATAAGAACTTTGTTTATGTTACGTGATGATGGTAGTATCTTT TTTAAAGAAATGTTCACTGTTGCCACATTTCATCAGTGCTATAGTGTTTCTGTCTATACTTCCTGTGTCTGGT CAGTCAAAATAATATTTTTTATCCCAAATGGACATTAATTTATGATTACTATGAGTTAATTGGCAAACAACTT GTTGAATTTGCTGATTTTTTTATTTGCTTTTGAGAAAAAAACAAAGTGCTTTTAAACAAATAAGCAGCATCTT AGTGGTACTGCATTCCCAGGGAGGACACCACCACAGCCAGGAACTTCTGAAAAGCTGCCTGGACATCACCAGT GAAGGCTTTACCCAGCTTTGCAGCAACCACAATGGTCAGGCAGTCAGACAACAGCTGCAAGACAACAAGCATC AGAAAACATTAACATGAAATGCGTTTGTTGAATAATGGTTGAATATGGATAACAGTAGTTACAGTTCCACAAC TTTACCTTGAAATTGTCAGGGTCCACGTGCAGTTTCTCGGAGTGCAGCACGCTCAGTTCTGCATATGTGGCCT TGATGTCGTCCATGTTCTTCACAGCCCGGTCCAGACCGTGGAGGATGGTTGTTCCGTGTTTTGCGACCATCGG ATTTGCAATGATAGCAGCGGCGTTGTAGAGGTTTCCAAAGTTGCCGAAATACCTCTGAGTCCAGGGGTAAACG ACCAGACACCTGAGGGAACATGCAAAACATTTTACTGGGTATGATAATGAGATTAAATATGTAGAGAAAGAAA GAACATTTTTGCGTACAGGGTATAAATAATATTCATTATTAATGTAAAAAACAAACCAAAACAAAAAAACATC TGACCTGGAAAGAGCTGCAGGTCCAACGACCTCATACTCCATCTTAGAGAAGATGTCCTGGATGGTGGCGCGC TCGAAGTCTGACCATTCAACCATTTTGCCGACTGTAGATTTTCCTGTGCAAGTCAGTAGATCCTGTGCTCGTC GGCTCCAGAATCAGCTTTTAAAGAAACCCTACACCCCTTCGAGAAACAAACGATTGGGTGGGCCGGAAAGGCG ATGTGGGATTTGCACCAATGATAATGGTTATCTGCGCCCCTCATATTGTTTCTGAGACAGAAATAGAACACAA TTATCTCTGTTTATCTAGTGTCAGCATACAATTCATGTCTTGATGAGAATAAAAATTGATAGTCTAGTACAAA TTTGATGGGCATTGTTTGTTTTACTGTCAGAATAAAGCTGACTAGCCCTCACCCTCAACTTCTAACCAAACTG CATCATATTGATTTAGTTACTGTACGGTTTAATGACTGCAGTATTTATCACTTGCTTATCACTTATTCCTGGC TTCATGACATAATTTAGGCTTATATTATACTATATACTATATTATAGGCTTATCTGTGGGCAAGGCTAACAAG 
TGAAGTGGAAACAAACTCAGGGTCCGTGAAGGCCCCATGTGCATGTCCTGTAAATTGTTTTGTTGTTATTTGA ACTGTTTGGGAATATTCGCTACCAAAGCAAAATATAAACGGAGGTATACGGGCTTTAGCGTGCATGTATTGCC TGTCCTGTACTAATATCTTGTAATATTTACATGATGCATATTCCTAAAAATAAATGTGTGTTTATTGACAAGG GTTTAATATGGTTAACTTGCCATGTGTGTGAAATCAGTAAGCGATCGAAAATTTTATTTATTTTCGTTTATTT ATTTATTTGATTTTGTTATTATGAATAGATCATGCACGGATCATCTCAGTGATACAATGTTTGCATACAAATT AATTAATGGACTACAACAGTGCACTAGCTTACAAAATGATCGGGGTGATGTGGTCCAATCCCCATTGCAAAGG GATTATCCATAAAAGTTTCATTCAGAAGAAGTGACCATGTATAATAACTAATTTCTTATATTCTGTGTTATAT CCTGCGGTTTAAATTCAAGGCTATAGACAAGAACAGCTACAACCTTTAACTGACTGAAAAAACTGACACCCAG GTATATTTTTGTTTTTGTGTTTTTATGAACAAGCACCCATCGAAAGGGGTGTTGACATTGTTGGATTGCATGC CACACACAGGCAGTCGATGCTTCTGCTTGTTGTTGGAGTTTAGCGGTATTTCTCAGACAGAGTCCAAGCCAGG CACTGTAAGAACACCTCTGGACCTCTGGAGTGAAGTCTGCGGGGAAGAACATGGCCATAATCAAGATGACGTT GTCGGCCAGGATCTGCGGGTGGCCATACACGTACTTCTGAGATGGCCCTTGTTCTGCCTTGTTATTGTTGTGA AACTCATTAAATACATCTCAAATTACTGCATAACTCTTTGTGTGATCATTTGTGAAATATATTTTTTATTATC CTCATTTCGACTTCTGTCAGTGTGTGTTTTCCTATCTGAGAAATTATTGTAACGACCCCGGGATTCTGATACA CGCTTCCCCTGATTTTGAAGAAAATTGGGTTTCCGTTATCCTACTGTCAAACAAACGGCACCGACAACATAAC CTACTTGGCGGAGGTAAAAACTTAATACACAAAATGAAAACTTCCTACAAACAGTTATATGATCCCAGTGTAA TTTAGTCGAATTTAAATTTGCAGAGTCAATCAACTTTATGGTTATTTTTCTAATCACTGGTCACTCACCGTCT CTGATTCCCTGCAGTGTCAGAATCAGAAATATTTTATTGATCAGGGTCCAGATGTATAAACTATTCGTACACA CAAAAATGGCACTTACAAGTTTCCAAATGTATAAAAATCCACAGCAGGTGAGAATAGTAGTAGTCCTTTTAGT CTTATAATGTGATATTGCGAACTGCAAAAAAGGGAACGACAAAAGTGTGAATTTGGGTTTGACACTTTATTCA GAGTCTTTTGTCTTTCACTTCACTGTGTTAGAACTACAATCTGTAGTTTAAATAAAAACTTCACTAACATAGG GATGCGTGTGTGATTAACAATCTTTTTGACTAGCCTTAATTTCGTTGATAAATATTCAAATAAATACATGATC TTTTAAAAATTATATTATTGTCCTGGTATTATTGAGGAAAAGAATAACCAACACCTTCCTGTGTGTGAGCAGT TTTGGGCAAGTTACTTTTAAAAAGTAATTAGTTACAGTTACTAGTTACTTCTTCCAAAGAGTAACTAAATTAG TTACTCAGTTACAAATTATAAAAGTAACTAGTTACTTCAGATAGTAACTATTGCGTTACTTTCAAGTACATTT CTAAATGCTCAAATGTGACCCCAACTCCACCCTTCTTTAACGGAACTCAATGCATGTTCAATTATTTATGATG AATTTGAATACTATAATGAGATGGACACTTAATACAATACATTATTAACAGAAACAATGTACACAAGTCTAAA CTATTTTAATGTTGCTGTGGGACAAAGTGAGACTAGCCTCCAATCAAATGCCATGTATGTAGATATTATGTTC ATATAGTGGATCGGTACAATAGATGTTTTGGAACTTTTGATATTTATTGCCCCTCAACAGGCCACAACTGGGC AAAATTAAATTATGCTTTTTAAGCACATACCAAAAATAAATAACAAATATATATACTCTTTGTAGTGCAAATA AAACAAAACAAAAACTTCAGACAATTGGTAGATATACTGTACTTCAAATATTTTCTTCCTCGAAAAAGTAGAA CACTGTAACAATATGAGGTGCTTTCAATCTAACTGAAAAGTACAATGTATAGCAAAACTAAGGAATCCAAATA TAAATAGGTTATACATAAGAAATAAAAAATACATCTCCATAAAAATAAATCTCTTTTCTGGCCATTGACAATC TCACTTCAAGAGGCTGCAGTCAGCCAACAAATACATCTAAATTAAATTATGCTCATTAAGCAATGCACCAAAA ATGGTTTCAAAAAATATATACACTCTTTTTAGTGCAAATGTAATAAGATGCAACACAACTCGCCATACCTGTC TCTTGTTGACTGACTCGCTTGTGTTGTTCGTTGTGTGTCCGACTATGCGTGCTTTCCAACACCCGCCCAACTC TACCTCTGATTGGCTTACCATGAAATTTTACTCAACCTCAGCCAATCGTCAGCATTTATGCGCTTGTCTCGTG CACTGCCCACTAACCAAGGAAGAACAGTAAAAAAAAGTCTTTGCCTCTCAGCTCAGAGATCTAGTTTGTCTGA TAAAAAAAACAGCTTCAATTATAGTAACGCGCCGCATTTTATGGCAGTAACTGGAACAGCGTTATTAAGATGG GAAGAGTAATCAATTAGATTACTCGTTACTGAAAAAAAGTAACGCCGTTAGTAACGCCGGTATTTATAACGCC GTTATTCCCATCACTGTGTGTGTGTGTGAGTGACCAATTAACAGGTCGTTAAAGAACACAAAGGCTGTAGCCG TGCGTAATAGATGCCAAATGATACAAATGCCTGGCATATGCAAATCAGACAACTGAGACCAGGATTTATTGGC TACATAGACCATATTTAAATAACCTGCATAAAGGTGTTTAATAAAAGCACTGCTTACTCTCGTATTTACAGTA TAAATGTACAAAATGCTTTATATTTCAAATTTAATAACATCATTTGGAAAGACGTAGACATATGTAACACGTT TAAAGTGTATATTCAAGTTGATTGAAAACATGTGGTCACCAACAGATTCAATCTTCTGTTTAATCGACGGCAA AATTTGTCACCAGGACCATCCCTATTGAAGACAGAATTGAATTCTAACAGTAGTTCAAGCCATTTAAAAGGGG CATGTTCAGGTCTGTTTGTTTGTGTAATGCACTTGGACATTCAACTTTTTATTTTTGCCAGGTAACCTTGAGT CATTATGACGACGACTTTATGGCAACAACCAAGAAATGTTCAAGGTGCATCACAACCCTGCATCACCAGCAAC AATAGGCCATCTTCACTGCTACCGTCTCCTAAGACTCAACAAGACTGGGAACCAAAATAACCAGCACTCAAGT ATATCAGTAAAACACTACATACTGTATCTGCAGTGATCTTCACACCATTGTCAAAAGATGTGCAGGGGTGCTG CACAATTGATGAACTTTACCTTTTAATACGATGTGGCTGTGCAGTCTTACCTTATCAATCCTGTACAGCCTCC ACCTTTCAGGAATCTAAACTGTTTAGGTGTCACATGCATTAAGTCGGCGTGAGCTGTTCATTTTTCCACAGTT GTGAACAACAAATATCAGTACAAGTTGTTAGAAATGTCGGAGCATGATCTTTTCAAAGACAAGTGCCTGGGAA ATTGTGGCATCTAGCGGTATAGTTGCAGATTGCATCTCGCTTCACCTTCCAAGCGCGAAGTACGAAGTACAAA GTACGGTGGCTGGGAAAGATGCGAACGACCCCATCTACAGCCAGCGTTTGGTTTGTCTGTTCTGGGCTACTGT AGAAACATGGCAAACCACCCTCATTCTAAGGCAACAAAAACACAACGAACCTTATTTCAGGTGATTATACATT AATGAAACATGTTGTATGTGCGCCAATAGATCATCCTAAATGTTACACACTGGATCTTTAATATTTATGTTAA GTGTATTTCTGAAAAACGTTCATTTAGAAAAAAAAACATTTAGACCGTTGAATGTTTATCCAACCTTGAGTTT 


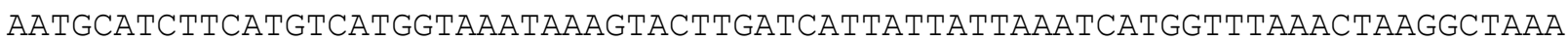
ATTATAGTTTTATATGTTATTTATTTTCTGTTATTCAATGGTTTTTGTGTTTTTTCATTTCTAATATTGTGAG

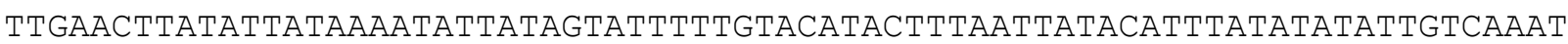
AAAGGGCGTCTCTGTAGAAGGTAATCTATGTTATTTATGACTCAGATTAAACAGTGTAGCTGGTGAAGATAAG TTAAAATAAAAATGTAAGTTTAGTAGACGTTTGAATTCATACTTACACACTTTATATTCGGGAAAACAGACTC TCTGGCTCATTTGAAAGCACACTGCGCAATTTATGTTGATTAAAGGGTGCGGTCATTTTAATTTCCTGATAA TGGTGTGCAATATGGTGTGTGTGCCGATAAAAAAAATGTGTAACGTCAAGAAATGAATATCAGCTTGTTGTTT TAGCCCACTAACACTACGTGGCTGTTCTACATATATTACCACCCACTTATTGTACTATTAATGTCCTTGTCAT CAACATATGAAGAATGGTGTCCTTTCGAGGCCGATGAAAGGCCAATAAAGTCCTGTGGAGTAATTCTGTGTTA TAACGgAGTTGTGCTGCTGTGTAACAGAAACCTTGTCATTATGTGGCAGTGGgTAAAgCATGCAACAAATAAA CCCTTGTCCATGGTTTAATTATGGGGTTTAAAAACAAATTTAGCTGCTTGGGGACATTAAGAACCTGTTGCA TGTAGGACATTTTCGTTATATTAAGTTGACCTACAGATAATTTATTTGGGTGGAGGAAGTCTGAATGTTCAGA TAATGAAAGCTTTGGATAATCTCAGTTTAACAGCCTCGGGCAAGTAACTTTGCATGGCCAGGGTACATAGGAA AATGGAgGGAATGTCTTTTTGAGAAGA GAAACAAATTATTAGCTCTTTATTCTGTGAGATATAGGCATAAGT TCTGATGTATCTGCATGGCGCTGACTGGTAACCATCACAATCCTTTAATGTAGTGTTGGCGCATGTCTGACTC TCTGCTGGCTTATCAATGCGTGTCATTAAACAGAGGAGCTGGTCACTCTTCAGTTGATATTGATTACATTGAA ATtATGGTTGA GTGGACAGACAAGGAGCGCAGCACGGTCAGTGCTATTTGGGAAAAAATTGATATTGATGTGA TTGGACCAGAAGCTTTGGCAAGGTAAGCTTAGAGGAAGTGATTTTGAACGAAGTGAGGTAGGCTGTTGCTTTG TTTTTGGTTTTTTTATGGTCTCTTTATATAGGAAGAGGCTTATTTTAATATTATTATTATTAAGTTTGTTTG ACGATAAAATCTCAGGATTTATATTAGGCCTATTGTTTGCTTAATGTTTAAATTTAAAAGATCGATCGATCAT ACTGCAGCTTCTTATTTATCAGTGGCAGGTTGTCTTCCTTGCATCACAACTGTTAGAAAATAATGGTATGAGG GGCAAATCTATTTGTTAAATAAAAGCTACTGAGGAATTAATGTTTGAATAAAGATATTAATGTCAGAGTAG ACTAATGCTTAATTAAAATTTGGTGTTTTTATTTCTCATGTTAGATTATTTGATATTTTCAGCACCAGGCGCT GTCGTACAAGATATTAGTTTTTAGTGTTGCTGCGTATAAATTGTCTGAACTCGTCCCACAAATTTTGATATAG CCCATTTTTGAAATATTTCCACCTTTATAACAGTTTATATCATGTAAGTCAACACGGCCTGAATGTAATTGT GTTTAAGCCTCAACCAGAGTAATACGTCTATCACTGAGTATTAAATGTCGTACATGATCTGTAAAAGTAATAA CCTTTCGTTCTTTTTGCTGCTTTGTGGAGTCAAGATATTTCAGACTCACATTTACGATGTGGGCTCATAACAA ATATATTTACAATACAAAAATCCACAGAGTGTTGATCGTTTACCCCTGGACTGAGCGGTATTTTGGTACTTTT GGAGATATCTTCTCTGCAACTGCTGTTTTGGGTAACGCCAAAGTAGCAGCTCATGGCAAGGTGGTGCTGAAAG CACTGGATAGTGCAGTGAAGAACATGGACAACATTAAAGCCACATATGCTGATCTGAGCCGGTTGCACTACGA GAAACTCAGCGTGGACCCGGACAACTTCAAAGTAGGCAATCTTGTCCTAAAATCAAAAGGAGAAATATTTTAT CAGTAGGCTACTGTAAGGTCAAACCTGTCTGGATTCATTGTGTCTTTTTGTTTTGTCCACAGCTGTTGGCCGA CTGCATCACAATTGCCGTCGCCTGCAAACTCAAGAGCGCCCTCAACCCTCAGGCCCAAGCTACCTGGCAGAAG TTTCTGTCCGTTGTGGTCGACGCTATGAGCAGTCAGTACTTCTGAAGAGCCGAACAGACCCAGCTGAAAAAAA ATAAATTGCATATTTAAACCCTGTGTGTTTGTCTCAGTGGCAGACATAGACTGTACCTGCTGGCAGGCTTAAT TATGCAAGGTATCTGAAACCATTAAAAATATGCTTTAGTACTCGAATAATTGCTTAAATGACCAGTTTGCATA ATGTTGCAACGTCAGTATGTGGTGCACTGGTGAAAGTCATTGCACCTTGACAGTAGTACGATATGGTATACTT TTAGTGGCCTATTTTCTGTACTAAAATGTGTATATATTTATAGACTGTATAAAGTTATCTGGGTTTTCGTAA GTGCCTTTTGTTCACTCTTTCTTGTTATCCACAGAATCTACACCCATTTGTGGATGGATTATGTAACAACAA ATAACATCTCTGCTGGGTTCCCTGGACTATGCTGTCCAATTATGTAGCAGTAGGGTGGTAGTAACATGAGAAA AAAACCCCAGGAAAACATAAAAACAAAACATTACTTAACATATGTCCATTAATTGATGACATATTGCTAAATG AGACACAGAATGGGAACAAACATTCTCAGGTCCTTACCTCTATAGTGCTAAAAAGACACTGGATATTATGAGG TCCTCTCCTCAAGAGGGTCATATCACAACAGGACATTTATGTTTTGTAATAAGAAATGTATACTTATGAAGGC AтTTTAGAGAACCTCCCCAATAGTCACAGTAGGTGTTGAGAGTAAATCAAGGAAATAAGAGCTTTTATGTCAT CAGTTTTTCAAATGACCCTAATCTCAGATTTAGTTATATGTATGGCTTTGTTTAAAAAGGTGACATGAAGCG AAAGTGGGAAGAAAATGTATCCATTTCCCGAGACAATTGACCAGTGATCTGATTTATACCACTCAATCAACGG TCTAACCAAAATACACCATTAGCCTCCCCACCAGAGAGTTGGTGCTTTTGTATACAAACACGCTGTAAGTGCA GATGCAGTAAAAGGCAGGAGACTAGATGAGCTAAAAATAAAGTTTTATTGTCTTGGGAAATTAATACATACAT CACAATTAGATAGCTGAAACTGCTGACACAAAGTTGAGCTGCTAATGATTTGTAAGAAAAACTATTTACAGGG AGGCAGCTTGTTTTACTAGTACAAGGGTGAATTGAATGATTCAATATGTTCACTTGCTAGAGCTGCCTTTAGT CCTTGAGCATGGCCTGAAGTTAATACCACTTGGTGGCAAAACCTCTTTGGTTTGTTTCTAGGGTCCACATACC TTGAAACATGATAACTCTTTCACAGTTTTTTGGGACAATCATTGTTTTTGCCATTATTGCCCTAATGTACTA TCGCCAATGTTATtgtAAgATCATATgtACCAAgTtTAAATCACACATACACAATATATATATATATACACA CACATCCACAAAGAATATCAATTGGTTTTATGAGTTTTTCACTTTCTCACTTCAGAGAAAATTCTGACCTGTG CCCATGGTATATTGGTTACTATTGACAAAGGCACTACCAGGTGCTCACAGCTCTAGGATCAGGACCCAGGCTG TTACTGTCACATGTGCTTTGTTGTGGTTTGTCAGCCTATATCTGAATTATTATGCTTATATAGTGCAGGAGCA TGACTCTTCTTGCAATATTTAAAGATTGAGATGGATTGAAAGACCACCAGAGGGCGCTCCAATTTAATTACAT ACGACCATGTACGACCAATGTTGTGCTCCAACGGGCTCAGTTTAACTCTGTTGACAGCCAGACACATTTTTCT 
CACATGAATTACAAGTGTAGACCTACGAAAAACTCGATAAAATAGTGGAGAAACAAAGTGAATGCAGATGCTT CCACACAGAAATGGAGAAAATACNATCCAATCATGCTTTGTGGCTTGTATATGTTGGATCTCTGTGAATAATT TTATAAGGAGTACGGTCTAGACCTGCTCTATTTGAAACGTGTCCTGATATATAGTTGCAGTGCATAAACCCCT CATTACAAAGATGAATACGCATATGAGAGTTCAATGAAGTTTCCATTTAACTAATCATCCGTCTGCAGGTACC CCAGCATTGTGTAGACTATGCTGAACTGTTTCTGTGTTATGTACAGATATCAACCTATACTGGTGTAGACATT TTACTGCATCAGAAAACACTCAAAACTATATTAGGGAATTGTGCCAATATCCAAATCACTGCGCAGGGAGTTG CTGGGTTACTGCCAGCAGTACTAACGTCATTTAGCAGAGAACTCCGATTTTCTTTATCAAAGCAAATGTATTG AAGAAACCCCACTGAACACAAGGGGATTTCTGTTGGATAAACTTTATTTTTTCGACAGCCGCTTAAAGGGTTA TTGCATGTGTGCTTGGGCATGGTTAGGTCTTGGATAGGGAAAGTATCGCTTCTAAAGTTGATTAAAGGTAATA TCATTTTGGAGGGTTTCCAGGCGAATGGGAATTGTTGTGTCAAAAAAACAGTGAAGACATGTAATTGCAAACG AAAGTCAAACAGTTTTTTTGTTTTATGTGTCTGTTCCATGCAGTTTTTGGGATATTTTTGGGGATATTTTTA TTAACCATCTCGTAATTGTGTTTTTTTTCTGTCATGCCAGAGGTCATCGACAGGGTCTTATGTGATTCCGAG AGGCTCAAGTTGAAATGAGGGTTTGAATAATTTCAATGAGCCACTTGGATGAAAAAAGTATTACTACATTTAG AAGAAATACAGAAATTAAAATTATAACCTCGTTTTGCAAGTAGAACACAAGAAGATATATTTTGAAAGAGGT TATGGCTGTCGTGTGTTAGTGTGGTTGAATAGATTCTGTATTTACCTGGCAAAGGTGAGAAGTCCATTAGACC TCTGCAGTGACGCTCAGCATCTGCTCATTCTACTCTGGTGTTTTCTGACTGTGTGACAGTGTGTCTCCAGATG TGAACAGATAAACCCTTATTGTTTCACGCCCCTGCCCTCAATTCTCTGCCCCAGTGACCTTATGTGAACATAA GTCAGCAAAACAGCTGAACTGAAGCTAAAAAAAGGTCGAGTCAGGACAAGTTTAAAGTTCTTTATTTTATATG CACAACAATTCCAGTGAAGCAGTTGTAATGAAATACATTGTTCTCAGGCTCCCTTCGTCCTCACACAAAAGGT AGAAAAAGAAAATCACTTAATTAAAAATAGAGAATCAAAGACAGTATTACAAACTGAATCCAGTCTAATTTGT GGAATAATCTTCAAGCCCCAGTGAATCTAAGCAGCATTCTAGTGCAATTAAATGAATGAATTTTCACTAACTA CAACTTCTCCCAAATAGAGAAATGTTAGTGGTGTTACAGTCTGAATCAGCAAAGCTACTTTTAAAATATTATC TTGTTCCCCTCTGAGCGGACCCACCCTTTGAAGGCTCCAGTTCTCCTCCAGATAAGCGTGTTGGCAGCAAACT TCACCCCAAATGACATCACTTTTCATGTTCAACCGATAACTAAATCCATTAATATTAATTAATTGCAGAGCCT AGATTTCAAATCAGTACGCCTGGACTCTTAGATGTTTACACCGTTGCCTATAGCTTTAACTTTCCATACTCAC ATATGTCTGTTCTCTGAATCAGAGCATTTAGACCCAGTTATTTTACTTTTAGTGTATTGGATCATATTTTGGT ATTGTGCTGTAAACAATAAAACGATTACAAGCTTTAATTTAGTATGTCATTGCTGAAATAATATAATAATTTA TTTGATTATTATTTAATCTAACGATGTAAGGACAAGTCATGTTGGATTTGGTCCAACTTGAAAGTTATTTAT AGAAGCATGCCACTTCAGGGTCGTATCAACACCCTAGATTTTGCGAAAAAGCTCAAAAAGCGTTTATCTGGTT AGGCTCAAATCTGATAAATCTGATCAAGAATGATATTAAGCTCGGGCACATGAGCCCATATTACTTATTACCA GTTAATTTAGTTGGAAACTATAATCTTTCTTTTCTGACGGGTTCAAGTTCGTCATTTTCGATGGCTGTATTGG GTATTTGTGTTTTGCATTAAAAGTATGCAATCTGAATTGAATTGAATTCTTGAGTTTGAAAGAATTCTTGAGT GAAAAAACAACTAGAGTGAAAACATGTGCTACATTACTTATTTTTAACTTGTGTTTTTTTTCACGAGTTGTTT TTTTGTCTTTATCTCATGCAAGCTGCAACACATCATTTGGAACCTGTCCAAATGCCACGCAGCCTATCTTGGA GGTGGGATGGGTGTGACCCTGCTCTGTTTTATGTATAAAAACCCCCACGGTTTGGGCTGATCACAGCGTTCAA CTTCAACCAAACAATCAACAGGCAAAATGAGTCTCACTGCTAAGGACAAGGCCGCAGTCAAGGCCTTCTGGGG TAAAGTGTCTGGCAAGGCGGAGGACATCGGCACCGATGCTCTGTCCAGGTAATTATGACCCCTAACTAACTGT AACстTTTTCTAAAATTACTGCATGTTTTCGTTATGAATTAAAATTGTTTATTCACTTACTCACATTTATTA TCCAGGATGCTGGTGGTGTACCCGCAGACCAAGACTTACTTCTCCCACTGGAAGGACTTGAGCCCCGGCTCTG CCCCAGTGAGGAAGCACGGTAAGACTGTGATGTCTGGAGTTGCAGATGCTGTGTCCAAAATCGACGACCTGAA TGCTGGTCTCCTGAACCTCAGTGAGCTGCATGCCTTCACTCTGCGAGTGGATCCTGCCAACTTCAAGGTCAGT GATGGTGATTAACACTTGGTAGTGTTTTCAAACTGCTGTTATTTTATTGCCTTCGCTCTGCCATAAGAAGGT ATGCACAAAACGTTTTAATTAATTCAAACAAAAACAAAAАATATTAAAAAAAACCATAAACAATAAAAACTAA

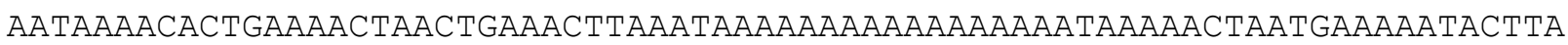
ACTATAATAACTGGTGTGAAGCCAGCTCAGCTTTCATTTAACTGTGTTTAAATTCAAAATTGAGTGCACATCA GTCGTCGCTGTGAGAGGAGAAAGAAAAGAAAGGGGAAGATGAAAATCAGTGACACACTGAATGTTAGTTTGGT GTTGTTTGACCTTCTTTGCAATATCTCACTTGTGGTTCTGGGTCTTTTGATATTGAGTCTTATACACTATACA TACTTAACGATTTTTAGGTACTGGCGATTGGATTAAATGTCATTATTCTCTTTTGACAGATTCTGGCTCACA ACATCCTTGTGGTCCTGTCCATCATGTTCCCCGAAGACTTCTCCCCTGAGGTCCATGTGGCTATGGACAAGTT CCTGGCTGCCCTGGCTCGTGCCTTGTCTGAGAAATACCGATAAACTGCAGGAGCAGGAGCAGATGATGCACAG CAGCATGGGCTTGGATGCACTCTGTCAGTCTGAATATCAATAAAAGATCAAATGCAATTAAACAGTTTGTGTG GAGTAATTTCTATCGTGCATGTTTAAAAGTGTGTCAAGTCAGGTCAACAGCATACATATTTTAGTGGAATTT GAATTGGTATAACGCATGTCAACGACGGTTCAACATATTTACTTTNAACAAAACAGACAAAATAATTCGTACA ACAACATAAAAACCCTATTTTAATCGACCAGAATGAATGCAAATGAATGAAGTATGACACAACACCCTACAAA TGTAAATCTCCAACGGATCTAAGTTGAAATTATATTACAGCCCTGAGGTGCAATTCTCTTTCTCTATATATAT СTATAACTATATATAGTGGCAGTAATATAATTTCTCTCTCTCTCTCTCTCTCTCTCTCTCTCTCTCTCTCTCT стстстстстстсGстстстстстTтстсстTстстCACTCATTTTAAAGGTTTAGAAGGATAATTATAGTTA

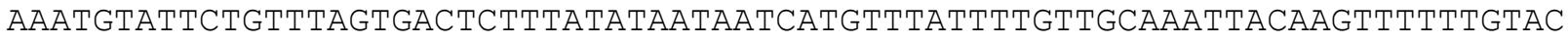




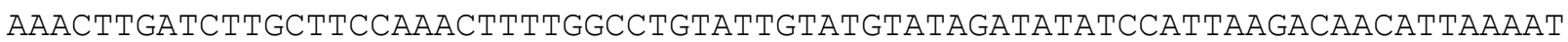
ATCTGAGTTTAAATAATGTTACAGTCACTGGTCTATTAACTCCCCTTACAGTGACTTACAGTGCAAGATTTTT GTGCTTTATTTGAACATGTTGGCCTGTCTTCTACCCCACTACGTTTCAGGGGAAATGTTATAATAATGTAGTA ATAATAATGCATTATAATGCGTCATAATTTTACTTTTTTAAAGTGGTCATTCTGCATAAAGAATACCTTAATA TTAGGCTGATAATACCAGTGTATTTTAATTTATTCGTATTTCAATGAAAGACTTTTAGTTTAATGTTATTAT TGGTATTGAAGCTTTAAATGGATTTAAATAGTTCTTCAACCACTGAACATAAGTAAATATATATTGTGAATAA AАСтTTтАСААтTСАATTGTATGCATCCTAATATGACCATCAATCCAGGCAGAGGAACACGCAACTTGTTGA AтAтGстTAтTGTTTATTTAтTTATGCTCTTGAGACATGATCAAAATAATGTTTTTTCACAAATGATCAGCA TGTGATGGTCTTCTTTCTCTCTAGTGGTACTGCCTTCCCAGGGAGGACACCACCACGGCCAGGAACTTCTGAA AAgCTGCCTGGACATCACCAGTGAAGGCTTTACCCAGCTTTGCAGCAACCACAATGGTCAGGCAGTCAGCCAG CAGCTGCAAGACAACAAGCATCAGAAAACATTAACATGAAAGGAATGTGGAATAATGGTTGAATATGGATAAC AATAGTTACAGCTCCAAAACCTCACCCTGAAATTGTCAGGATCCACGTGCAGTTTCTCGGAGTGCAGCACACT CAGCTCTGCATATGTGCCCTTTATGTCGTCCATGTTCTTCACAGCCCGGTCGAGACCGTGGAGGACAACTTTT CCGTGAGCTGCAACCATCGGATTTGCAATGATAGCAGCGGCGTTGTAGAGGTTTCCAAAGTTGCCGAAATACC TCTGAGTCCAGGGGTAAACGACCAGACACCTGAGGGAACATGCAAAAACATGTAGTATAACTCAAAATTGTTA CAGGCTACTTCTTTTtGGCATTCATGTTAAGGAGAAAATACAACAACAGAAAAAATCTTTCACCTGGAAAGA GCTGCAGGTCCCACGACCTCATAGTCCATCTTGGAGAAGATGTCCTGGATGGTGGCGCGCTCGAAGTCTGTCC ATTCGACCATTTTGCCGGCTGTAGATTTGTTTCACGAGTCCTGTGTGGGCCATGACCAGATGCAACTTTTAAA AGAACCCTTTCACTCCTCCCAAAATATGCAATTGGATGGAGTAGGGCAGTGGGTTGTCCATGTATAGCTACGG ATTTCCAAAAATGATAAGAAAATGACAAATCTGCACTGCTTAGATTGTATATGAAGCGGAAGAAATAGATATT GCTGGTTGTGTGAATTCATGCAGTAAAAAGAAGACCTAAGCTGGCAGTATTAAAACGAGATACTGTTTAAAAA TGGGGCCAGATTCTTACGAATTTTTTGAAATGCTTGAAAAATGACAATTTGTACTATCTGACAGTATTAAGTA GCCTACGTTTTTCTGGTCTTCTGGCCACTCAAAGTGCTTTACAACACTTGTCACATTCATTCACCCATTCACA CAAACATGCATACACTGCTGGCAGAGGCTGTCATGCAAGGAGCCAACCTGCTCATCGGGTTAGGGGGTCGATA GCTTCCTATCTAAAGCAATTTGGGGTTCAGTATATTCTGTCAAATCAGTCCAAGGACACTTCTAATGGATTGG GGGAGCCGGAAATCGAACCATAGACCCTCCAATTCATAGACAACAACCTGAGCTCAAAAACGCGAGCCACAGG CGCCCCCTAACGTGGATCTAATGTGAATAAATGATATCCAAACTTGAGGTAATAAATCCAGTAGATGGCGCTC CTACACGTCAGGGAAACTGACCTCTGCAAGACCGGTTCAAAGTGAGGATAATGCAGACCAAAATAAATGATGT TTGTAAAATCACAACTGAAAAATGACCTTCTTTGAGATCAGTTTAGCTTATGCATTTATGTATCGATCTACAG TTTACACAACAAAAAAATACTTCGGTGTGACTTAGACGGTAATTATCATTTGACGTCATTGTAATCATTCACC TCATTTTTTCCCTCCCAGCTCATTTTCGATGTTAAGAAACGCAGGACCTGTGTGCAATGTACATTCATACATT AAAATTATATATACTTGTAGTGATTTACATTATTTATGGGGTATACCCAAGTTTTATTTTTCTGTTGCAACAG AATGGTGGCTTATAAGGGTGGCTTATTTTACGCCAAGCTGTAAATAATGGTGGACACAAAGGTAGCCATATGA AgGgAgGTATACTCAAGATTGGGCAACAAAGACAACTGACAACATACAGTATGTTTAACGACAGAAACAAAAG GAGGAGTTGTGTGAGGTACAGGGTCCCAGGCACTAGCCTCACTTCAGTACCACGGACAGCTCCCTGATCACCG ACTGCCACACCCCGCGATGTACTGTCCTACTGGCATGTTTCTGTTTCTTTATGAACAAAATCGTCTTACAAAA CAGTTGAAAGCATTTACTTCATTACCACACTGTAACTTTCAACGATTAATGAGCGACTTAAGTTCTTATAATA TAATAATATAAAAAATATATATATATATATATATATATATATATATATATATATATATATATATATATATAT ATATATGTTTCTCTGCACTGGCTCCCTGTAAAATTTAGAATAGAATTTAAAATTCTCTTCCTTCCACATTCAA GAGTAGGCTTAAGACGTTCCTTTTTGATATAGCTTATGGTTAGGGCTGGATCAGGTGAGTCTTGAACCATCCC GTAGTTATGCTACTATAGGCCTAGACTATCCTGGGATTTTCCCATGGTGCACTGAGCTCCTCTCTTCCTCTCT CTTTCTGCACTCATTCATGTCCCATTAATGCATGTTACTAACTTCACTTCTTCCCCGGAGTTCTTGTGCTTTC TCGTCTCGCAGGTTCTTATCGATCCTGGTGGAGCCTCCTGCCATGGTCCTGCTAGATTGCCTTTACCAATACT GTTGTTGCTGTGCACCTGTCTGTCTGCCTGTCTCTCTGTCTCTCTCTCTGTCTCTGTCTCTCTCTCGGTCTCT GTCGGTCTCTCTCTCTCTCTGTCTCTCTCTCTCTCTCTCTTTCTCTCTCTCTCTCTCTCTCTCTCCCTCACCC CAGCTGGTCGAGACAGACGCGGACCCACCTAGAGCCGAAGGATCTGTCCTAGGTTTCTGCCTTTAAACAGGCA GTTTTTCCTTGCCAAGTGCTTGCTCATGGTGGAACTGTTGGGTCTCTGTAATTAATGTTTTAAAGAGTTCGGT CTAGACCTGCTCTATTTGAAAATGTCCTGAGATAACTTCTGTTATGAATTAGCGCTATACAAATAAAATTGA ATTGAATTGAATACCTTTTAATATTCATGAATGCCACATGTCTCCTTTTCTGCACAGGCAGAAGTTCTTACG ATTTCCTTGCACAGAAACGTTTGGTGAATAAGCACTAAGCAGGCAGGACGAGTGCAGCCCTGTGACTGGCTAA AGTGAGGAGGTGAGAATTAAGTGACAGCACAAAGTGTTCATAATGTTACTGGGAAAAATCCCTAACGATTAAT GAGAATAATATTGTAATAACAATAATAATAAGAAGAAAAACATTATAACTTCAATAAAACTGGTTACCTTGAA ATCAGTATTTTAAATACGTTTTATTATCATAATTATATTATTATTATTATTATCATTATTATCATTATTATTA TTAтTATTATTATTATTATTATTATTACCTAAATGTGTATGTGTACTGAAAAATGTTTAAAAAAAAGAAGAGA CATCTGTACATTTTATTGTTTTATTCAGCAAGCCCTTCCATGTTAAAAGGGATGTTGGCGTTGTTGTGGACG ACATGCCGCATTGTATGCAGTCGATGCCCCTGAATCCTGGATCGCTGGAGTTTAGCGGTATCTCTCGGACAGA GCCAAAGCCAGGTTCTGCAGGAACTTGTCAACGGAGACATGGACTTCAGCAGTGAAATCTGCGGGGAAGAGCA TGGCCAAAACCAAGATGATGTTGTGGGCCAGGATCTGCGGTTGTGCAAGAAACACATTATTAATTAAAGTCAT 
CAACAAAGAAAAAAAAACACATGGACAGTTAGATAAACAGTTATGACATAAATTCACATTTGAACATGAACAA AтTTTATCTGACTTTTGGCTAATGATGACCTAATTGTATTACTATAAATTAAGTAACTTACCCTGAAGTTGG CAGGATCCACTCTGAGCTTGAAGGCATGCAGCTCGCTGAGGCTGATGAGACCACCAGCAAGGTCATCCATCTT TCCGACGGCGTCGCCAACGGCCGCCATGATGGTTGCACCATGGGCTCTCACTTGAGGGGATTCAGGACTGAGA TCAGACCAGTGGGAAAAGTAAGTCTTGGTCTGCGGGTATACAGTCAGCATCCTGTCAAAGAAAATAAAGAAGA ATCAATGACAAGAAGTAATAGCGCGCATAGTTTAAACTATGACAAAATACAATATAAAAAAATAACAACAAAT AAGCGCTTACCTGGCCAGAGCCTCAGCTCCAATCTCGGCAGACTTCGGGGACACTTTGGCCCAGATAGCCTTC ACCACGGACTTGTCTTTTGCGTTGAGACTCATGACTGCCCTTTCTTCAAGTTTTCACAAGTGAAAGAAAATAT TCAAGCATGAACATGCTGGTTAACGACCTTTTTTATATCAAACTTATTTGCACCACACCCCAGTATCAAATCT AAACCACACCTTCCTCAAAGAAATTGCCAAAGTTTCCCAGCCAGTGTCTGAGATAAAATTAGGCTAACCACTC CCTGTTCTTGTATATATTTTCAGTCAGTTTTTTTAGGTAATTAAAATATAATGGTTTTGATCTGTCTGC AGATATAACCATATGCTGAAATGTGCATTCAGTAAGACAAAAGACAAGACTTTGTAAGGTTGCATTTATTCAA CAGTTTGGACAGAATAAAGAAAGAATAGAATAATAATAAAAATAATTTCCACAGGTTTTCATTTGCAGAAAGA GACATTGAATGGTTATGGTTAATTGTGCATTTATGCAATTATGCATTTTCATGAAATAACACTTAAACTCTGA TATAATTGATTCAACGTTACCATAACACTAATGACAATTCTGCAACTTAAACAAACATAAAATCTAAAACCAA ATAAAAACAACTACAAAATTCAATACATTTATCATGAAAACAGTGGCAAAGTACAAACTCTGTGACCAAATGT GACAAAATGAAAAAAAAACCTATGACCTAAAAGGAAAGGACAGTGTTACAACATTAACATTAAAGTGTTTTTT TCATAAGATGTTTAGTTTTCCATCCTTTTGTAGCCCATAAACACAGTGTAACATAAAATACTACTATTGCAAT GGAATGAAAGATCAGCAAAAGACAGGACAAACCATATACTTTTTTACAATTCTGCAATACACTTTAATACAAT AAGTTATTTTAAATTCAGAAAAAATGTTGAAACGCATTTCATTGAAAATTTTAAGTAACACAAACTCAATTTT GCAAGTTAAAAACATTCTTGATTAAGTACTTTTAAGGTACTAGGTATTTTGTTTTCGAATAAAAATTACAAAA TTAAGTCAGTGTCACTTAATTAGCAAAGCTACTGATCAAGGATGTTTTTTAACTTAAAAATGTAGTTAGATTT ACTTAAAAАСАTTATTAAACAGTTAAAAAAAGTTTTTTAGTTAATAAACTTAACTGGTTTTAATGTGATAGG TAGAGAAGTTAGAAGATTTTGCAACCTTGCAACCTATGAATTAATGAACATTTTTAAGGAACCGATACAAAAC TTCCTTTTTATTCGGAAAAAATGTAATATCAAATTTGGCCACATTTATTCTTATCAGCTTTTTTTTCAGTACT GGCAAACGTCCCATCTAACTCATTATAGGTAACAAGCCAAGCTCTCGTCTCTAGTCCAATCAGACGCTTCCAT GGCTGAGAGGTGTGGCATTGAGTAATTAATAAAAACCTCCAGAGGACTGGTGTTTGCAAAAGGTTTGTGCTCA TATTTCCTGTCTGAAAGAAACGTGAGAACCTTAACGTCGTCAACATGGTCAAGTGGACAGATGCAGAGCGTGC CGCCATCACAAGCTGGTGGGGAAAGATCGATGTGAATGAAATTGGACCCCAGGCTTTGACCAGGTGACCTGTA TAATCTTATTCATAGTTTTTTTTATTTCTTACACGTTGACATTACTAGATGTTATTACTACTAGACTTTCAA CAGTCTGTTGGTGTTGTAGTTTGACTTTTTTCTTTATCATGGATCGTGTCATTTCAGACTTCTGATCGTGTAT CCGTGGACTCAGAGACACTTCGCAACATTCGGCAACCTCTCCACCAGTGCCGCCATCCTCGGAAACCCTTTGG TGGCCGAGCACGGTAAGACAGTGATGGGTGGGCTGGACAGAGCTGTGAAGAACATGGACGACATCAAGAACGT CTATACCAAGCTGAGCGTGAAGCACTCTGAGAAAATCCATGTGGATCCTGATAACTTCAGGGTATGTAACCAT CAACACCCAACTTCTAATGACTTCACAGTTTATTCTACTAGACTATCACTGATCTCTGTATTCTATTCTTCTC ATTTTAGATCTTTGCTCAAATCATCAGCGTGTGTGTGGCTGCCAAGTTTGGACGTCAGTTCACCCCTGATGTC CAAGAGGCCTGGCAGAAGTTCCTGGATGTGGTCGTCTCTGCCCTGAGCAGACAGTACCACTGAGGATCCGGAG TGAAAGTTCACATTCAGAAAATGTGCAGCAGTTCTACAGGCGTTTACAAATGTCTCAAGCACTGCGTTGAAGC TTTTGCCAAAAGCACCAAATAAAATTTAAATCAAATACGTCTCTGTTTGTGTGTGATTGTCTGTAAAACTTTC TTTTGTTTTTATCTTTTAGCCATTATTTTCTTCTCCTTAGATGTTCGTAGCTTCAGCTCACAACCGATTTACA CTAGGgATTTACATACATTAAAAACTATTTTAAAAAAAATACAATGGTGAGACACATTGATCCCCGTGTAATC TGGTCTCTGTAATTACAAAGTCTGCAACTTGGGTTTTTGTGTATAATTCTTTTAACACATTGTGAACAAAGT GCTTTTGATCTGCCTTTTAAATTCCCACATCATAATCAGTTATTTTGAGACTAAATCATCCCTATCATCTCAA TTTTAACAATTTACGAGTCTGCCATAACAAATATAGGGCTCTGATACCAGGTAAAAAATAAATAAAATAGAAT TATATATGCACAAACACATATATTTAGAGAGAGAGAGAGAGAGAGAGAGAGAGAGAGAGAGAGAGAGAGAGAG ATATTGGGGTTTCTTGTAAAGACAGCCTTCGCAGCCTTGGATCATAATACTTCTTGTTATAGTAATTATACTA ATTACTCGATATTGTGATTTTACATTCTCAAGTTAGGGTTTAGGTTTTAGAAGACACACATAATTGAGTGGTT CAGTGGTAAATGAAATTATTTGCTATGACTCATCTTTAAGAAGGAAAATACTTCTGTCGGTTATTTTGGCTTC TTTAAAATGTGCAGTTTGATATAAATGGGTGTGGTCACCTGAAATTAATGATAACAGTGCACCAAAATACTGT ATCATGCAGGCGAGAAATTGTGGCAGTGCTGCCAACTTATGACTGAACAGTTTATTAAAACATCGCTGGATGG GCTTTTTATTTCAAATTTAAAATATTTAAGCAATTTAATAGGTTCACCATGTGCATTTGGGCCTAAATGTTTT AAAGCACACATCAACACCACTCCCCTCAAGAAAGAGACTAAAAAAACAATAACACAAAAAGAAAACAAAAACA AGTATATACGGGTATAGGCTTATTTTAATTACAATATTTTAATTCCAGCAGTGTGCACACAAGTGTACTGAC TAAGCATGAATTCCACTATTAGACTATTATTGACATTTACAATTATATTAAAGTCGATAGTCCTGATTAGTTT

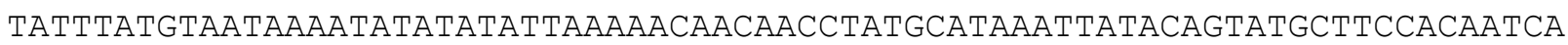
GCTAAATGCTAATCCCTAGGGGATATAATGTAACATATATAGTATTTGTCAGAATGCTGAAATTATCTTTTAG GTAATTTTAAACAGAAATAAAAAGAGCACATTTGAACTGAAGATCCATTTGATTGCAAAACTGTGAAATACAC TAATGGTAAAGTGTGTGAATAACAATAGTAATTTGTAATTTGTTAGATTTAATCATACTTAGAATCATCTTTT 
TAACCTGTTGGATATTTGTATATTATTTGTGTAGCCTACGTTACATGACGCTTTATTAGATCTCTCTCTTATG TAAGTTACACGTTACTTTTTAATAACAATAACTTAATTCATATAGCACCTTTAAGAAAAGCTGTGATACATTG TTGCAGCAATGGTTGTATAATTACATTCATAAAATAGATTAATCTAGATAGAGTAGGGCGTAACCATGCATTA TTTGAGGGGAATGTGTCCAGTCTGTCCTCAGCAATATACAGTAGAAAATATGTTAAATATGTGAGCGCTGTCA ACGGATTTGCCTCTTATTATTTCCTATTTATTTACAATTTATGTCCATTTGTTAAAGAAAACATAAGTGTGAC TCCCCCTCCCAACTTTATTCTTGGTGTCGCCCAAATTCAGTGCGTTGGTTCAGTGTGGCCAACTTTTTAGATC СTCTTTAAATACTTTATTTCTTAAAACCCACCAGCAAAACGTTAAGCAGCTTATTCAAACCATCAGGGAAAAG ACAAAACATTATAGTATTTGGGACAGGACTAGAGCTGCTCATCTGCCAACAGCGTTAATCCCTAATAAATAAC AGAACAACAGAACATCAAAACAAATGTCAGTGTAGTCATCAAAATGTTCTATATGTGCGCATTTTAATTTATC GTTGTAGCTTTTTCTGGTGGAGTGCTCTCATTTGGACTCATCCAAAGTCTATAACTTGGTAAGGCCCTTGTAG AATTATAACTGGTAGGAAACATATACTGTCAGTGGTAGGAAAGCTATAGAAGCCCATTTCTGCCAGGAAAAAA AAAAGACAGATAAAACTTAGCCTTGATAATAAAAATATCCTCAAAGTAAGTTGAAATTATGAGATAAAAAGTC ATAATTAAGAGATAAGAAGTGCGCATGTGCCAATGGCAGTCAGCAATATATTCACAGATTCGCACTTCCCGCA TCAATAACTCATAATCGGGACATTTTTAAAACACAGCGGATGCTTCTTTCCAACCGTAGTAAAGCACACAACA CACAAAGCTACAACCTCATTTTCATCAAAACAAGCCGTCCCTATGCGCTGCTCTAATAACATTGATCTCTATG TAAGGAGTGCGCAGGGACCGTCAACAATTTTCAAGTTGTTGCTCGTTGTGTGCTTTACTACGGTTGGAAAGAA GCATCCGCTGTGTTTTAAAAATGCCCCGATTATGAGTTATTGATGAGGGAAGTGCGAATCTGTGAATATATTG CCAACTGCCATTGAAGCGCATGCGCACTTCTTATCTCATAATTTCGATTTTTTTTTAAAATCTCATAATTATG ACTTTATATCTCATAATTTAGACTTACTTTGAGGATATTTTTATTATCAAGGCTAAGTTTTATCTGTCTTTTT TTTTTTTTTTCCCTGGCGGAACTGGGCTTCCATAGAAAGCTCCGATCTGCTGAAGCAAGCGGAGTCTTTTAGC CACGGTCCCTGTTCCTGCCGAACATTTTTTGAAGTGACACAAAACAATCGCAACAGTACACGGATGTGAGGTT GGGAGTACTTAATTAATCGCCAAACCTCCACATTAAAACAAGTTAGCCTGTAATTGGTCCAGGGTCGGTGGTA TGTGTGTATGGCATACATTAATACGAGCTTCGCCCGCAACAAGCTAACGTAACTTACGCTGTTTGGTCCTAGC TACAGCTCACGGGGCAGCTAACGTAACGTTAGCTAGGATGCTAACATGATGTTCAACCCAGTAGCCGGGTTAC AAGGTGACCGAAAATCCATGTACAGCCAGTCACAAAATAGCGTGTACAAAACTGGAGACAAAACAAGCCCTAT TAGCGTCATACTGGTTAGCTCTGGCAGCCGAGGACACAAACTACTTTTTCGATACCCTTTCCAAAGAGTGGCT GAATGTCCGTCATCTCTTACAGGTAGGGGTAGCATCCAGTTAGCATTCACCAGCGAATCACAGTACAGCACTG ATGTTTGTTGATCTTTACTCAAGGGAAACTCGTGCCCTGAGGTCTCACTTTCTCGCAATTACACATACACAGC TGGATAAAATTACGAACACCCCCCAATGTAATGCAGTCAAATACAGCAGCCTAGCAATATATGGTTCCCCTAC AATGCTAAGGCCACAGTTGTCCACTGTTCCTCTTTCTGAAAAAGAAAGTTTTCCTGTTTGGTTTTTTTTTGCA TTCCTTTTGTCATTGTGACTGTTTGAGCTTAGTATGATATATGTAAATGTACTGGATGTAACTGTGATTGGGC TACTTCACATATAGTTTTCGTATGCTTTTTGTCCACACCCAGTGAAACCCAGCTGTAGAGACAGTCGCACTAA CTGAATACACACTTACAGTATTTCTGACGTATTACTTCTGTATAACCTTCATATGTGAGCTTCCATGCCAATA TACTGTGGGGTATATTGGCATGTGTCTAAAATGGCAAAAAAATAAATCAAAAATGACATGTCACTCACAAGTA GACCCTTACTAAGAGAAAAATACCCATAACAACAACAAACTATGGCTTGATACAGTAACACAACACTTCTCTA ATACATGTTTGATATTCAGAGTGTTGTTTTAACATCTTGTTGGCTTTTAATGGGGCTACATCTCAAATCAGTG TATTTTTACTAGTTGGCATCAATAATCAAGTGAACTAATACTATTATTTCTGTCCACAAGCTAAACAACGAAG TCCATATGTACTGAACACAACCGGGGATGGTCTTGAAGATCAGGATGGTGATTCAAGGTATTGTTTTTGGTCT TCATTTAATATTACTGGATTTATAAATTAATTATAGGGAAGTTGACTCTAGGTGGACACACAGACAATTCTTA GATCAGCATGAGGCACTCACTCTCATGTGTTTCCTCCCTGTGTCCCCTGTTTTATGTTTCAAAAACCTCATTG TATTTTTCAAGAGGAATTGAGGAGTGTTAAAAGGTTGTTCTAACCCATGTGGTGTGACCTATATTAATGTCCT CAAAATGAACTTACAAGACAAAATGCTCACCATGTGCCTCTTCAGATTTGTCATATTCCCAGCTGCCGTTTGC TTATCTGAACTGGTAATAAAAACAAGCATTTAAACCAGTTCTGATTACTTTATTTTGTTACCTCCGTTCATGA GAAAATGGAGACAAATTTCTACATGTGTTAAATAAACAAGTTTATACAATGCACACATGTTTTCTAAGCTCAA CATGAATGTTGGGACAAGAAGCTGTCAATCGTTGAAAATAGTCATATTACAAATAAAATTGGCCATTGATTCA AAAATAGGATAGTGGCAGTGCTGCATCATTTCATGGTTTTACACAAGCTAAGACATGAAAAAAGAGGGAATGA CTTGTTAATCCACTGAGTAAAAAAAGAAATGGGAAAACTGCTGTACACGAATGTTGCAGATATGACAATTGTT AAATAGAGTAGTATTCAGATCAATCCACAGTCACTATATAATATTAAATATTATTGTATTATACTATACTCTC TTGTTTATGGTATAGACTCTCCTGGTGGTGAGCCACATGAGAATAAATATAATTAAAAAACAGCCTCAAACCT TTAAACTGGTGTGTTATAGATATTGAACCAACTACTGTATAATATAAAATTCAAAGTTGAATATTTCCTGCAC AAGCTGTGTGAGTTTAAGCTAAAAATAATTATACAAATGAAGATATACTCACAAAAATTTAGCTATTCATGAT TTTGAGTTGAGCAGTCACTCTGGCAGAAGTTGTATGAAACATGAACAGAATTGGCAAACAACTTGAACTCCTA CCATTTGCACTCTCGAAGATATTGATGTCTGATTCTGAAAAATACAAAAATGTCAGACAGAGAACCTTTCAGT CTTGTTTGTTATTTTCCAACTGCATTCAGTTTTCATCATGCTGCGGCTTTATGGATGGTAATGCTAGTCAGTC GTTGGGTGGTGATATTACTTTGGTCACAGGGTTGTAAGAGGCAGCCACAAATGATCATTTAAACTCTTTTATC AATGCAACTCACTAGGCGTAATCAGATAAAACTGACACAAGATCATGGAATCTTTTTTTTTTTTTTCTTTTGC TCAGTTTTTCCATACGGACTCCACTACAAACATACTTTGTACTATGTACTCCAGTTTAACTTTCTTTAAAATT AAGGTCTATGACCTTTTAGACAGATTATACTGGGATATAGAACCATACAGAGCCTTATAATCATTGAATAATT 
AATTAATATAAGGTAGGTGTTCTATGGCCCGTCATTTTTGGCTTGGTTAACAACCTGACAGCAGCATTTTGAA CAGTTTGAAGCAATACAGTAACAGCTTTATAAAGACCAGTATAAAGTGTTTTATAGTATTTAAGTGGCTCATT GTGAGGGATTGCATAAAATATTTTCACGGTATCTTTTTGAGTAATGTTAACAATTTTAGTTGCCTTTATGACA GTTTTAAGCTGGCATTTAAACTCATGTGGCTATAATGTTAATGGCATTAAGGCACAGTCAGGGTAAACAAGTT AATTTGTTTGAATCAACCTGCAGACCAGCATTTTCACATCATAGTATCACATGTTTGTTCGGGTTGTTTTGAT GTCCATTTTATGCATTCAGCCCAACTGTGTGTTGCATTTTGCTTTTTGTTTGCTTTTTTCTTCTTTTTTATGG CCCAATTCCCATTTCAGAGAACAATCTCCACTAACTGACGAACAGTTGGTAGCAGGGTAAGACCAAATCATTC TAAACCACCTTTCCTTCGCTTAGATCACCCCTGTCTTTTTTTTATCCCCTTCTTTCCTATTTTGTGTCCTATA TGGATCCCCAACCCTAATTTCAGTAAAATCCTTGTAAATTATCAGTAGCAGATCCAGTGAATCAGTGCTTTGT TTAATTCAGTATTTCAGTTTTTCTCTCCTGTATTGTATGCCTTTATGAACAATAAACAATCCAACAGTTTTAA ACTATAATTACAGTTTTAATTCACATCAATTATATTACCTCTAGTATGTAGACTCAGCATTACGAAACATATT GCAAATCAAATGCAAACTACAATAAAGCAGCTACTACACATCCTTTTCAAATTGTGTCAGTGGGGTAAGTCTA TAATGTGTTTAATTTTTTTCTTGAAGGTAACGCCTTGATTTTTATTCCTTTGATTGCAGCATGAGTTACTTAA GTCAAACCAGCATGAGTCTTTCACATTCTTCATTCATACAGCGACTTCACTGTTTCTGCAAGAAGGCCTCTTT СTCTTTTCTCCTCTCCAGTAGCCACACAAGCCTTTTTTATTTATACTCTCATTTTTGAATTACTTTACAGGCA GATGCATCTGATTAACGCAACCACTCCTATAGACAGGAATGTGAAAAATGTAAACAAACAGAGCTAATTTAAA CCCTCTTACTTGTTCCAGCTGTTTCTGTGCTGTAACCTCTCATCCCATCTTGCACACTTTTTGTATTGAGTCT GTTCTTCCTTCTTAAAGGTTCTCTGACATCATCCTTGCCACCATCCTGGCCACCAAATCTGACATGTGTGGGA AGAAGTTTGAACTGAAGATAGACAATGTTCGATTTGTGGGTCACCCTACTCTCCTTCAGCATCCTCCCATCAT TCAGGCAAGGATAAAATGTCTCCTATTAAAAAATCATTTTCGATTTGAATTGAACTTCCAGTAAGTTTAAAAA GAAATGTTATGCAGTTTTCTTTTGGACAGTTAGCTTGTTACCACTGTTCAAGAGAAAAGAAAAGATAAATGGA TTTGCACAGTGCAAGAGAACAACAAAATGAGTGTGAAGAATCCCTACATTGAAATGTTTTAATTAGCAGGCAT TGCCATTAATAGCTGTTATTATACATGGCAGTAGACACCTTTGATTGATAATTTCAATTAAAAGGTTAACAGT TCCATTTTGAATTTTATTGTTATCATTATAATATTGATGTGTCTTGCCATCTAACTTTGAATAGGCTATAAGG CCATTTCTCATTGTCCCAAGGCCCTCTAAATGTATGTAATCTTTGAACTTTGGCAGATTAGTCTTAACTAAAA CAGTGCCTCCTACTGACGGTTTTCTATGCTTCTGCACTGTCAACAGCTGTGGCTGGAGGCATTATCTTATTAT CTGCTTTTTTTTATAAATTTCACCAGAACAAGATCAACAAAGCTTAGCAAAGTCAAGTAAAATATATTTTAAA TATCCCTATATCACACATAAAAAAATTGCCTCAAGGGTTTTGACAATCTGCACAGTAATCCTCTATACTTAGA CTCTCAACTTGGTTGGGGAACAACTCCCATTAATGGGCAAGATGTAAGAAACCTCAGGAAGGGCAACAGAGGA AGGATCCCTCTACCAGGATGGACAGAAGAACAATAGATGCCACATGTACAGAATAGACCAACATTATGGCATT CACTCACAATGGCAAGATTACAGATAGATTGTAAATAAATACGAAGAATGGACCTGATGATGAGCAACCCCAG GTGTCACCAAACAGATCTGAGCCACACAACCTCCACTCCACTATTGAGACGTGGAAGAGGACGGACTACAAAG ACTCACAAGAGCATAGCTTCACACACATCATTTCACACAGGTTGTAAAAAAAAAAAAAAAACACACAGACAAA GGGAGAAAGAGACAAAGACAACATGCACAAAAAAAAAGAGAGAAAGACCAAAGGAGAGGCTGAATGTCCTGGA GAACGAATATCAAAATCCATAAATCTACCGCAAGGTGGTAATTCTGGTATAATGTAGTGTAATAAATAATAAA GTGTGAAGTAATTATAAATTGCTTCTACAAAAATAACAGAAACAGTTTACATTATTCTGTAATGCAACAGCAC CTACTGTACATGACTTAAAAATGACCATTTAGCTCAATCAACTCTTCTTTGAGAAAGTCTCAGAAATTTTTTT TTCAGGTTTGACAAAGGTTGATTTATTGCAGAACTATTGTGCTAGGTTGTATAACATTTTGCTGCTGCAGCTT TTATCATGTTTTTGTTTAATATATGTGAATTTAACTGCTCTAGATCTACACACTGTGGATTTACATTTTAAAA TGTATTTATAAACACTTGGTTTATTACATACATCTTATTCATTTTTTTTTTCTTTTTATGCAGGTTTCTAAGA CAGATCCTTCACCTAAGAGAGAAATGCCTACAATGATATTGTTTAATGTGGTGTTTGCCCTAAGGGTATGAAC TTTTAATAATACAATTTTAATTCTTTGGTTTTATTTTGTTCTGCTTTTTGCTCTACCAGTTGAAAAATGCTGC ATATAAGTGGTCATTTTAATATTACAAATTAACGTCTTTATTTTAAATGATGGCACAATTAAGTCAGCTGGCA GTGGGTGGGACGCGTTGCGTTCTGTTAATCTGTGTGTTCTGTGAAGAAGGGTGTGTGTAAGTGATAATATTCT GGTATACTATATCTGTGAGAGCATAGGAACTCAGAGGGGGGAAGGGCCAATTTACTATGCTTAATCATGCTGG TCAACTAGGCACAGCCTGTTCCTGCCCATGCTGAGTCACTGTACTCAGTAAGTCCCAGTATAGCTGAGGCAGC CGAGGACACAAAATGCTCAGTCATGTTTTGTCAATGATATCTGCCCAGTAACTGTGGTATCTTTACTGCAGAA ATGTTCACAAGAATAACAGTTCCATTGGGTTATTTTAGCTTGTCAGTGTATTTGCAATTGGGTAATTTTTTTG TATTTTACAATAAATTCATAAGATTTAACAATTCCATTTTTAATGAATTCACATTTGTTCACCAGTAAATTAT GTATGTTATTCTACATGTTCATTCATACAGATAAGCTTTGCATTTTTGTTTTTATATCTACTTTGCAAGAAAA TTAAAGCAATTGATCTGCTTAACAATGCCATGAGATGATGCCAATGACCACCCTTATTAACATACCTGAGGAG AGCAGAATATTAGAAACTCCTCTCAGTATAAAGTAACACCACCATTAAGCTTCAGAAATACCACAGAGATGAG GCAAAAACCTTTCCAACACAATCTCAATAAAAACTGAACAATATACTTTAGAGTGATTGGATTTATCAGAGGT TTATTGGATTGGATTACATTACAAGTGATGATTTTTCAGGTGAAACTGATGAACTCATTCCGCAGCATTCTGT ATAAAATATATACATAAATAAAGGGGCTCAAAATAATTTAAATATTAAAGGCCATATATGTTTGATAGTTTAG CGGTTAACAGGCCAGTAATGACATGAACATTAACTGAGCGACAAATTAAAGCACTGATGTTGCAAATGCAGTG TAATAGGTAATAACTGAGTTGTATATGTATTACTAGCATATTTCTGTCAAAATTACACAGTTTAAAAAGTGGC TCCCTTTCCTCTTTTGCCCATAAAGGCGAATGCTGACCCGTCCGTCATCAGCTGCATGCACAACCTGTCACGC 
CGCATTGCAATAGCTCTGCAGCATGAGGAGCGGCGCTGCCAGTACCTGACCAGAGAGGCCAAACTGATGCTGG CCGTCCAGGATGAGATTACTACCATGACTGAGAGTAAGAAAGAAAGTTGTTGCACCTCACAGTTGACAGCAAT TTACTGCTCTGTCTCAGTTAATCTATAGAATTCCATAGAATCCATTGTACACAATGACATATTTATTCTTGAA CATATTATTGAGCTTGTAGGTACACTAACATTGTAATGATGTATTATAGTTGTGTTTTGAAAGTATATTTTCG TATATTTTTCCTGATTTAAGTGGATGGAAGCCCCCAGTCCCCATTTAGACAAATTCTTCCCAAATGTAAGCTG GCTAGAGACCTGAAGGAGGCTTATGACAGGTGTGTGATTAAACATATTATACATTGTTATTTCTGTTTTGTTA TTTCCATATTTTTCTCACCATGCAGAATGCATATTGTTCATACAGTTGAACATTTATGTTTTTGCAGCCTTTG TACAACTGGTGTGGTGCGACTACATATTAACAACTGGCTTGAAGTGAGCTTCTGTTTACCACACAAGATCCAC AGGATTGGTGGTAACTATATCCCCCCGGAGGCATTAGAGCGCAGCCTTAAGGCTATAAGGTGTGTTTTTACCT TCATGTGTACGGTGTATATATATATATATGTATGTGTTAAATATAACATTTTGTTTGTGGGTTGAAACGTTTA AAATTACACTTTTGGCAACACCCCAATCAGCAGTTGTTGACTGTGAAAGATACTTGGCAGCCCTTTCAAAGCA TAAACACATGAAGGGAGTGTTATCCCAAGTGTTATTACAGATATAAAAAATGTATACAGTATTTAAAATTATA TTCAGATATTGCATATAAATATTACATAAAACAATATAAAATTGCTTATAAATCTTAATTTACTATGTAAGAA ATGTATAACATTTACTATTAGTTAATATAGTTTCCTTCTTTTCTGCCCCCAAATACAACATCTGACAAACTTT TGACCACCTCCTATTTACCCTAATTTATAGTGTTCATTTATACTTCATTTAATTGTGAATGGAATCTAAAAGC AGCATAGTGTTAATGTGTTAATCTGACTTGGCAAGTCATGCAAAACTGCAAATGATAGGTAGGATGTCATATA TCATCCACCTGGTTGAGGAAAAATAGCACACATACGCTTTTATTTACTCAACCAGCCTGCATAGGCATACAGT ATACATGACTATTAAGTAGCACAGGATTGTCACAGTGCTATTCAATCTGTGTTGTTAGGTTCATTGTTGACCC ACAGGTTAACAAGAGGCCTAAAAACTGACAGTCTTTAACAGTATTATATTGTGAGTATTATGTCTTTAACAGC TGATTAAGGGCTGTGTATTTTGTAAACTTCAGTACTGAGTCACTATTTAAAACATCAAGAAACCCTGAAATTG TTTTTGTGGGCCATACTGCCCCCTATTGTCACCTCAGTATACCTACATAAGAGTTGTGATCAACCTCTACCTC TCCTTTAATTTCTGTCTTTTGAAGATAAACAAAATATGCAAAGGGTCATGTAAGTCATGAAATTGTAACAGCT GTGCTGATAATTATTTTTTAATAATCAGAGTGGTTTGTGTTCTCAGAGACACACAGTTCTGTACATGGATACA TGTATAGATGGTTCTGTTTTACAGGGTGGTACTAAGGCTGATTCCTTCTGGTGTCTATTGTAGAGGCAGTCTT GATCTCTAAAGATGAGTTTTGTTGATTTCAAGGCTGAAACATATGTTGTTTGATCCTCCAGACCTTATCATTC CCTACTGCTACTGGAAAGTGAGAAGGCACTGCTGAGCCAGCTTCCTTTGGACTGCTCGCCTGCGATGGTGCGC CTCATCAAAACCTGCTCAGCGGTGAAAAACCTGCAGCAACTCGCACAGGATGCTGACCTGGCCTTACTTCAGG TGCAGAACAGTCACATCGCCTGAATCACCAATGCACTTTACAACACCTGATGCCATTCTTTGAACTTGAAAGG CAAATTGGAAGTACTTTTTCATTATTAAACAAATTAATTCACAAGTGGATGTAATGATTAAGCTAATTTCAGC CATCAGTAATAAAGAGTTTGGTGATTGTATTGGACTTTTGTAATTCAAATACAGTACACAAATGTAAGTTAGG GAAGTAAAAGTGTATTCAGACGTTACTGTGTTAATGCGGTCTCACTGCCATCTAGTGGACAATATTTGTACAT TCAAAGCAAATCTGAGACTACACAAGTCTGAGTAGTATAGGATGTTCTTATATCTTCCTTCTACTGTTGGTTT TCAATTCCAAAACACTTTATTGTTTGATTGGTTTTTTTTCACATAATTTATATTATTTGCAAATCATTTTCCT CTTAGATATTTCAAATTGCTGCTCACTTGGTTTACTGGGGCAAAGCGATCATCATCTATCCACTGTGTGAGAA CAACGTCTACATGCTGTCTCCTCATGCTAACATCTGCCTGTGAGTATTCCTGATATGACATGAGACATTTGGC TGTACTGATTACTTCAGAGCTAATGAGCCATAATATTGCAGAGCACCCATCCATGTCATTGCTTCATACACAG TAGGTAAAAAAATATTAAATTCAGATTTCCAAAGCCTGT 
Supplementary file2: In order to validate the hemoglobin gene succession in the MN Cluster, PCRs were performed on genomic DNA from primers designed at different locations in the cluster.

Couple of Sequences (5'-3')

Primers (CP)

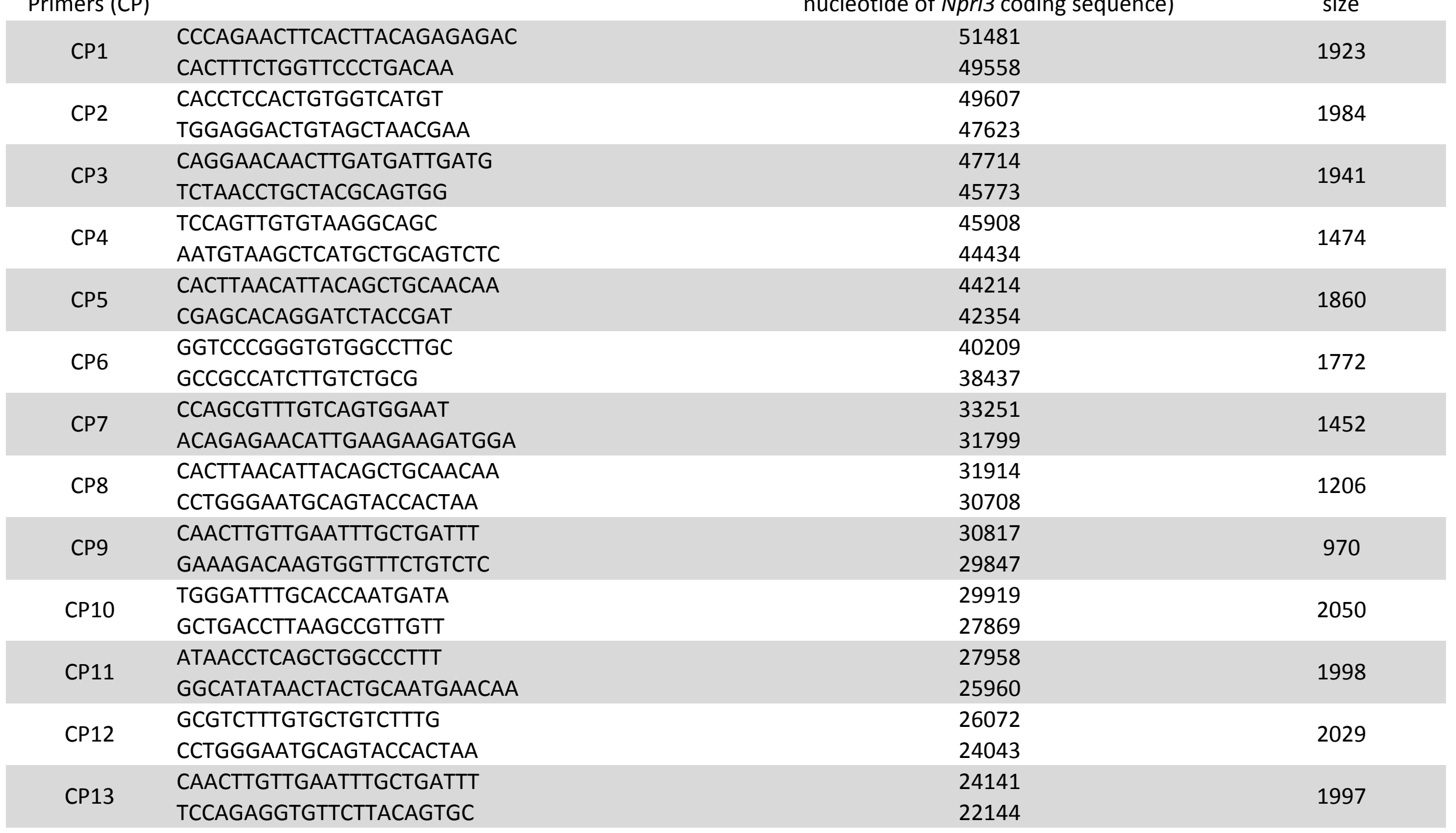
Positions in the cluster (related to the first predicted amplicon Nprl3 coding sequence)

49558

49607

47714

45773

44214

42354

40209

33251

31799

30708

30817

29847

27958

25960

24043

24141

1997 


\begin{tabular}{|c|c|c|c|}
\hline CP14 & $\begin{array}{l}\text { GAACAAGCACCCATCGAAAG } \\
\text { TATGGCGAGTTGTGTTGCAT }\end{array}$ & $\begin{array}{l}22284 \\
20346\end{array}$ & 1938 \\
\hline \multirow{2}{*}{ CP15 } & GCTCATTAAGCAATGCACCA & 20435 & \multirow{2}{*}{2014} \\
\hline & СCTTCTACAGAGACGCCCTTTATTTGACAA & 18421 & \\
\hline \multirow{2}{*}{ CP16 } & TTGTCAAATAAAGGGCGTCTCTGTAGAAGG & 18450 & \multirow{2}{*}{1910} \\
\hline & AGTGCTTTCAGCACCACCT & 16540 & \\
\hline \multirow{2}{*}{ CP17 } & CGCCAAAGTAGCAGCTCAT & 16591 & \multirow{2}{*}{1940} \\
\hline & GCACCTGGTAGTGCCTTT & 14651 & \\
\hline \multirow{2}{*}{ CP18 } & AAAGGCACTACCAGGTGC & 14692 & \multirow{2}{*}{1913} \\
\hline & AACTGGAGCCTTCAAAGGGT & 12779 & \\
\hline \multirow{2}{*}{ CP19 } & ACCCTTTGAAGGCTCCAGTT & 12798 & \multirow{2}{*}{1906} \\
\hline & GGGAACATGATGGACAGGAC & 10892 & \\
\hline \multirow{2}{*}{ CP20 } & TCTGGCTCACAACATCCTTG & 10933 & \multirow{2}{*}{1915} \\
\hline & CTGCAGCTCTTTCCAGGTG & 9018 & \\
\hline \multirow{2}{*}{ CP21 } & TGCGCATTCATGTTAAGGAG & 9082 & \multirow{2}{*}{1971} \\
\hline & AGAACCTGCGAGACGAGAAA & 7111 & \\
\hline \multirow{2}{*}{ CP22 } & ATGGTGCACTGAGCTCCTCT & 7229 & \multirow{2}{*}{1967} \\
\hline & CTGGGAAACTTTGGCAATTT & 5262 & \\
\hline \multirow{2}{*}{ CP23 } & TTCCTCAAAGAAATTGCCAAA & 5291 & \multirow{2}{*}{1922} \\
\hline & CACACACGCTGATGATTTGA & 3369 & \\
\hline
\end{tabular}


Supplementary file 3. $\mathrm{Hb} \alpha$ and $\mathrm{Hb} \beta$ amino acid sequences of the European sea bass and other teleost and non-teleost species used for phylogenetic analysis.

\section{$\underline{\text { Hemoglobin alpha sequences }}$}

>Anguilla_anguilla_Anodic_HbA

MSLSAKDMAVVKGFWNKIAPKADEIGGEALGRMLRVFPQTKAYFAHWKDTSPNSPEVKKHGALILATIGDVVNRIENMTTVL GSLSDLHAFKLRVDPANFKILGHNIMVVICMTFPNDFTPEVHLSVDKFFQNFTLALSERYR

>Anguilla_anguilla_Cathodic_HbA

MSLTAKDKSLITGFWQKISSKADDLGAEALSRMIVVFPATKVYFSHWPDLGPGSPSVKKHGKVIMAAVGDAVGKMNDLVGAL SALSDLHAFKMRIDPGNFKTLSHNILVACAVNFPVDFTAEVHVAMDKFLAALGAALSDKYR

>Danio_rerio_LA_Hba1

MSLSAKDKAAVRGFWAKIAPKGEQIGNEAFSRLLLVYPQTKTYFSHWNDLAPGSPSVKKQGKKIVGGLGLAVDKIDDLFNGLLN LSELHAFQLRVDPANFKLLSHCLLVVFAMLFPDDFTAEVHLAIDKFLARVALALSDKYR

>Danio_rerio_LA_Hba2

MLSSAEKELIAEIWDKMTPVAEEIGSEALLRMFTTFPKTKTYFSHLNISANSEHLRSHGKKIVEALAEGAKNISTLTTTLAPLSRFHA YQLRIHPTNFKLFNHCILVTLACRMGDDFTPVVHAAIDKFLSAFSAVLAEKFR

>Danio_rerio_MN_Hba1

MSLTAKDKAVIKGFWGKIASQADSIGQEAMGRMLTVYPQTKIYFAHWPDHSLGSAQVKKHGKIVMGAITDAVGKIDDLLGAL SSLSDLHATKLRVDPGNFKILSHNILVTLAVHFPADFTAEVHVAVDKFLAALSAALADKYR

>Danio_rerio_MN_Hba2

MSLSDTDKAVVKAIWAKISPKADEIGAEALARMLTVYPQTKTYFSHWADLSPGSGPVKKHGKTIMGAVGEAISKIDDLVGGLAA LSELHAFKLRVDPANFKILSHNVIVVIAMLFPADFTPEVHVSVDKFFNNLALALSEKYR

>Danio_rerio_MN_Hba3

MSLSDKDKAVVKAIWAKISPKADEIGAEALARMLTVYPQTKTYFSHWSDLSPGSGPVKKHGKTIMGAVGEAISKIDDLVGGLAA LSELHAFKLRVDPANFKILSHNVIVVIAMLFPADFTPEVHVSVDKFFNNLALALSEKYR

>Danio_rerio_MN_Hba4

MSLSAKDKAAVKTLWAKIAGKADDIGHDALSRMLIVYPQTKTYFSHWKDLSPGSAPVRKHGKTVMGGVAEAVSKIDDLNAGLL NLSELHAFQLRVDPANFKILSHNILVVLATLFPADFTPEAHVAMDKFLSALALAMSEKYR

>Danio_rerio_MN_Hba5

MSLSAKDKAAVKTLWAKIAGKADDIGHDALSRMLIVYPQTKTYFSHWKDLSPGSAPVRKHGKTVMGGVAEAVSKIDDLNAGLL NLSELHAFQLRVDPANFKILSHNILVVLATLFPADFTPEAHVAMDKFLSALALAMSEKYR

>Danio_rerio_MN_Hba6

MSLSAKDKAAVKTLWAKIAGKADDIGHDALSRMLIVYPQTKTYFSHWKDLSPGSAPVRKHGKTVMGGVAEAVSKIDDLNAGLL NLSELHAFQLRVDPANFKILSHNILVVLATLFPADFTPEAHVAMDKFLSALALAMSEKYR

>Danio_rerio_MN_Hba7 
MSLSAKDKANVKAFFDKVAPKAEEIGRETLSRTLFVYPQTKTYFSHWADLSPNSPQVKKHGTTVINGVLTAVGLMDDLKGGLLT LSELHAFMLRVDPANFKIINHNLLVSLAMMFPDDFTPEVHVSVDKFLAQVSLALSEKYR

>Dasyatis_akajei_Hba

MVLSSQNKKAIEELGNLIKANAEAWGADALARLFELHPQTKTYFSKFSGFEACNEQVKKHGKRVMNALADATHHLDNLHLHLE DLARKHGENLLVDPHNFHLFADCIVVTLAVNLQAFTPVTHCAVDKFLELVAYELSSCYR

>Dicentrarchus_labrax_LA_Hba1

MSLTQKDKAAVKALWAKISKSADAIGSDALSRMLTVYPQTKTYFSHWPDMSPGSAPVKAHGKKVMGGINLAVSKIDDLTNGL LELSEQHAFQLRVDPTNFKILSHCVLVVVSSMFPNDFTPEAHVAFDKFLTAVALALSEKYR

>Dicentrarchus_labrax_LA_Hba2

MLSKREKDLIAQIWGRLTPVADDIGSDALLRMFATFPGTKTYFSHLDISPRSAHLLSHGKKIVLAIAEGAQDISQLAVNLAPLQTLH AYQLRIDPTNFKLFSHCMLVTLACHMGDDFTPVAHAAMDKYLSAFAAVLAEKYR

>Dicentrarchus_labrax_MN_Hba1

MSLNAKDKSVVKAIWAKVSPKSAEIGAEALARMLTVYPQTKTYFSHWSDLSPESPQVRAHGATIMAAVGDAVGKMDDLAGG LISLSELHAFKLRVDPANFRILAHNIILVLAMLFPADFTAEVHVSVDKFLQNLALALSERYR

>Dicentrarchus_labrax_MN_Hba2

MSLTAKDKAAVKAFWGKVSGKAEDIGTDALSRMLVVYPQTKTYFSHWKDLSPGSAPVRKHGKTVMSGVADAVSKIDDLNAGL LNLSELHAFTLRVDPANFKILAHNILVVLSIMFPEDFSPEVHVAMDKFLAALARALSEKYR

>Dicentrarchus_labrax_MN_Hba3

MTSLTAKDKDAVRTFWAKVSGKAEDIGADALARMLAVYPQTKTYFAHWKDQSPTSPSARKHGITVMTGVADAVTKIDDLKGG LLSLSELHAFTLRVDPANFKILSHNILVVMAIMFPTDFTPEVHVAMDKFLAALALALAEKYR

>Dicentrarchus_labrax_MN_Hba4

MTSLTAKDKDAVRTFWAKVSGKAEDIGAEALARMLAVYPQTKTYFSHWKDQSPTSPSARKHGITVMTGVADAVSKIDDLKGG LLSLSELHAFTLRVDPANFKILSHNILVVMAIMFPTDFTPEVHVAMDKFLAALALALAEKYR

>Dicentrarchus_labrax_MN_Hba5

MTSLTAKDKDAVRTFWAKVSGKAEDIGADALARMLAVYPQTKTYFAHWKDQSPTSPSARKHGITVMTGVADAVTKIDDLKGG LLSLSELHAFTLRVDPANFKILSHNILVVMAIMFPTDFTPEVHVAMDKFLAALALALAEKYR

>Dipturus_batis_Hba

MVLSDANKQEIHHVAELIKAHAEAVGADALARLFELHPQTKTYFPNFSGYHATDAPVKAHGAKVINAVLKAAEHLDDLPKHLEK LATKHGHELLVDPHNFVLFSDIIVVTLATRLPTFSPATHRAIDKFLEELVHQLSSKYR

>Gadus_morhua_LA_Hba3

MLSKQEKELIIEIWTRLTPLADRIGAEALLRMFTSYPGTKTYFSHLDITPRSAHLLSHGQKIFLALAEGSKDIANLMTNLAPLQTYHA YQLRIQPNNFKLFSHCMIVTLACFMGDRFTPSSHAAMDKYLSAFSAVPGEKFR

>Gadus_morhua_MN_Hba1 
MSLTPKDKATVKLFWGRMSGKAELIGADALSRMLAVYPQTKTYFSHWKSLSPGSPDVKKHGKTIMMGIGDAVTKMDDLERGL LTLSELHAFKLRVDPTNFKLLSLNILVVMAIMFPDDFTPMAHLAVDKFLCALALALSEKYR

>Gadus_morhua_MN_Hba4

MSLTDKDKALIKGFFAKVSSKAVEIGHQTLARTIVVYPQTKVYFSHWKDLGPDSPNIRKHGYTVVKGVLDSVDLIDDLVGGLLELS ELHAFRLRIDPANFKILNLNLVVVLGLMFPDDFTPQVHVSVDKYLALICLALCEKYR

>Gasterosteus_aculeatus_LA_Hba1

MSLTDKDKATVKAVWAKISKSADAIGADALSRMLVVYPQTKTYFSHWPDLGPNSASVKNHGKNVMGGIALAVSKIDDLTAGLL ELSEQHAFQLRVDPANFKILSHCILVVIAGLFPKDFTPEAHVAVDKFFCGVSLALAERYR

>Gasterosteus_aculeatus_LA_Hba2

MLSKKQKDLIAEIWEGLISVADDIGADALLRMFASYPGTKTYFSHLDISHRSAHLLSHGKKIVLAIAEGAKDISQLTVTLAPLQTLHA YQLRIDPTNFKLFSHCMLVSLACHMGEDFTPEAHAAMDKYLSAFAAVLAEKYR

>Gasterosteus_aculeatus_MN_Hba1

MSLNAKDKDAVKAFWAKVSGKAGDIGSDALSRMLTVYPQTKTYFAHWKDMSAGSDPVVKHGKAVMGGVAEAINKIDDLNA GLLNLSELHAFTLRVDPANFKILSHNILVVMAILFPKDFTPEVHVAMDKFLGALSRALAEKYR

>Gasterosteus_aculeatus_MN_Hba2

MTTLSTKDKEVVKAFWAKVSGQGEEIGTDAVARMLRVYPQTKTYFSHWKDRSASSPDVQKHGRTVMAGVGDAVAKIDDLKG GLLNLSELHAFTLRVDPANFKILSHNLLVVMATMLPNDFTPEVHVSMDKFLAAVALALSEKYR

>Gasterosteus_aculeatus_MN_Hba3

MTTLSTKDKQVVKAFWAKVSGQGEEIGTDAVARMLRVYPQTKTYFSHWKDRSASSPDVQKHGRTVMAGVGDAVAKIDDLK GGLLNLSELHAFTLRVDPANFKILSHNLLVVMATMLPNDFTPEVHVSMDKFLAAVALALSEKYR

>Gasterosteus_aculeatus_MN_Hba4

MTTLSTKDKKVVKAFWAKVSGQGEEIGTDAVARMLRVYPQTKTYFSHWKDQSASSPDVKRHGRTVMAGVGDAVAKIDDLKG GLLNLSELHAFTLRVDPANFKILSHNLLVVMATMFPNDFTPEVHVSMDKFLAAVALALSEKYR

>Gasterosteus_aculeatus_MN_Hba5

MTTLSTKDKEVVKAFWAKVSGQGEEIGTDAVARMLRVYPQTKTYFSHWKDRSASSPDVQKHGRTVMAGVGDAVAKIDDLKG GLLNLSELHAFTLRVDPANFKILSHNLLVVMATMFPNDFTPEVHVSMDKFLAAVALALSEKYR

>Homo_sapiens_Hba

MVLSPADKTNVKAAWGKVGAHAGEYGAEALERMFLSFPTTKTYFPHFDLSHGSAQVKGHGKKVADALTNAVAHVDDMPNA LSALSDLHAHKLRVDPVNFKLLSHCLLVTLAAHLPAEFTPAVHASLDKFLASVSTVLTSKYR

>Ictalurus_punctatus_LA_Hba1

MSLSENDKQMVKTLWAKISPAADQLGCEALCRMFLVFPQTKTYFAHWPDLSLGSDSVKKHGKKVMDSVGEAVNNIDNLFGG LSSLSELHAFQLRIDPTNFKILSHNILVVIANRFPDDFSVEAHLSLEKFFARVALAMSDKYR

>Ictalurus_punctatus_LA_Hba2

MLSALEKQILIDLWHKLIPVAEDIGSEALYRMFTTFPKTKTYFGHLDLSHGSGHLRSLGKKIVVAIAEGTTHISTPLFTSSLGYLSRYH AYQLRIHPTNFKLFNHCMLVTLACHLGGDFSAIEHAATDKYLSAYSAVLAEKFR 
>Ictalurus_punctatus_MN_HBa1

MSLTDIDKTLVKAFWGKVSGKADAIGHEALVRMLLVYPQTKIYFSHWPDLSPGSESVKKHGRTVIGALNDAVVNIDDLVGGLSA LSDLHAYKLRVEPGNFKILSHNFLVTLAINFPADFTPEVHIAMDKFLAAVSAALADKYR

>Ictalurus_punctatus_MN_HBa2

MSLSAKDKAVVKDLWAKIAPKADKIGAEALGRLFEVYPQTKTYFSHWSDLTPGSPQVKKHGGVIVRKIGEAVGHIDDLTGALSSL SELHAFKLRVDPVNFKLLSHTIEVSIALFFPAEFTPEVHVSFDKFLQNLALALSEKYR

>Ictalurus_punctatus_MN_HBa3

MSLSAKDKAVVKDLWAKVAPKVDDIGAEALGRLFEVYPQTKTYFSHWSDLTPGSAQVKKHGSVIVRKIGEAVGHIDDLTGALSS LSELHAFKLRVDPVNFKLLSHTIEVSIALFFPAEFTPEVHVSFDKFLQNLALALSEKYR

>Ictalurus_punctatus_MN_HBa4

MSLSAKDKDTVKAFWATVSPKAGEIGAEALYRMLTVYPQTKTYFSHWTDLSFGSAQVKKHGKTIMASVDGAVSKIDDLTTGLLS LSELHAFQLRIDPANFKILAHNVLVVLAIMFPAAFTPEVHVSMDKFLNALALALAEKYR

>Ictalurus_punctatus_MN_HBa5

MSLSAKDKAAVKAFWATIAPKAEDIGGQALFRMLTVYPQTKTYFSHWNDFSLGSEQIKKHGKMVLSGVGEAVDKIDDLTNGLL NLSQLHAFQLRVDPANFKILSHNLLVVLALLFPADFTPEIHVAMDKFLAAVSLALSEKYR

>Ictalurus_punctatus_MN_HBa6

MSLNAKDKAAVKAFFSKVSCKAEELGNEVLSRTLFVYPQTKTYFSHWNDLSPGSVQVRKHGLTVIQGVLDAVERIDDLRTGLLNL SELHAFMLRVDPANFKIISHNMLVVFALLFPNDFTPEVHVSMDKFLAQ

>Lepisosteus_oculatus_Hba2

MSLSTADKDNITKTWAKVSPKAAQIGAEAFGRLLIVFPQTKIYFTHFADLSPSSAQVKAHGAKVMGAIAQAVSHLDNLSEALSKL SELHAYNLRVDPVNFKLLGHTLLVSLSASLGADFTPEAHVAWDKFLANIALVLAEKYR

>Lepisosteus_oculatus_Hba3

MLSQEEKDLLVSIWESIAPDAEEIGAEALLSRMFTCFPKTKTYFAHLDISPGSAHLRTHGKKIIMAVGDGVKNISTLATTLAPLSLLH AYQLMIHPSNFKLLSHCILVTLSCRRPQEFTPVAHGAWDKLLSAVSAVLAEKYR

>Lepisosteus_oculatus_Hba4

MSLSDKDKQHVQSIWSKASGRLEDIGNEALSRMLIVYPQTKTYFAHWSDLSAGSAQVRAHGKVIMTAVGDAVGHLGDLKGFL SHLSELHAYKLRVDPANFKILSHNILVALAIYFPADFTPEIHVSFDKFLAALSLALAEKYR

>Lepisosteus_oculatus_Hba5

MSLSDKDKQHVQAIWAKASAKVDTIGHEALSRMLVVYPQTKTYFSHWGDLSPGSAQVRKHGKVIMTAVGDAVGHLGDLKGF LSLSLALAEKYR

>Mustelus_griseus_Hba

MAFTACEKQTIGKIAQVLAKSPEAYGAECLARLFVTHPGSKSYFEYKDYSAAGAKVQVHGGKVIRAVVKAAEHVDDLHSHLETL ALTHGKKLLVDPQNFPMLSECIIVTLATHLTEFSPDTHCAVDKLLSAICQELSSRYR

>Oncorhynchus_mykiss_Hba 
MSLTAKDKQIVTAFFGKVSGKAEDIGNEALSRTLVVYPQTKTYFSHWTDLSPGSAPVKKHGLTVMGGVLEAVTKIDDLAGGLLTL SELHAFTLRVDPANFKIINHNILVVLAMLFPDDFTPEVHVSVDKFLAKLALALSEKYR

>Oryzias_latipes_LA_Hba1

MSLTEKDKAAVKALWAKISKSADAIGADALSRMLLVYPQTKTYFSHWPDTKAGSEPVKKHGKKIMGGVGLAVSKIDDLAAGLLE LSELHAFKLRVDPANFKLLAHCLQVVIANMFPKDFTPEAHVACDKFLANVALALSEKYR

>Oreochromis_niloticus_LA_Hba2

MLSKREKDLIKEIWERLTPVAAEIGADALLRMFASYPGTKTYFSHLDISPNSSHLLAHGKKIVLAIAEGAQDISQLTVTLAPLQTLHA YQLRIDPRNFKLLSHSMLVSLACYLGDEFTPVAHAAMDKYLSAFAAVLAEKYR

>Oreochromis_niloticus_LA_Hba1

MSLTEKDKAAVKALWAKISKSVDAIGAEALGRMLLVYPQTKTYFSHWPDLTPGSAPVVSHGKQIMGGVTEAMSKIDNLRGGLL ELSELHAFKLRVDPSNFQILAQTIMVVVAAMFPNDFTPEAHVAFDKFLAAVALGLSERYR

>Oreochromis_niloticus_MN_Hba3

MTSLSAKDKNTVKAFWAKVAGKEEQIGCDAVSRMLTVYPQTKTYFSHWKDLSPRSAPVKKHGATVMAAVTDAVSKIDDLTGA LLSLSELHAFTLRVDPANFQVLSHNLLVVLSIMFPQDFTPEVHVAMDKFLAAVALALSEKYR

>Oreochromis_niloticus_MN_Hba4

MTSLSAKDKNTVKAFWAKVAGKEEQIGCDAVSRMLTVYPQTKTYFSHWKDLSPGSAPVRKHGATVMAAVTDAVSKIDDLTG GLLSLSELHAFTLRVDPANFKVLAHNLLVVLATVFPNDFTPEVHVAMDKFLAAVAHALSEKYR

>Oreochromis_niloticus_MN_Hba6

MSLSAKDKETVKAFWAKVSGRAGDIGSDAVARMLTVYPQTKTYFSHWKDLSPGSAPVRKHGGIVIASITDAVNKIDDLAESLLT LSELHAFTLRVDPANFQILAQNILVVLATMFPVDFTPEVHMALDKFLGALARALSEKYR

>Oreochromis_niloticus_MN_Hba7

MSLSSKDKTVVKTFWGKVDSKSAEIGGEALGRMLVAYPQTKTYFSHWGDLTPSCPQVKKHGAVIMAAVGKAVKNIDDLTGHL SKLSELHATQLRVDPANFQILAHSIILVLGMYFPGDFTPEVHVSVDKFFNNLAWALSERYR

>Oreochromis_niloticus_MN_Hba8

MTSLSAKDKNTVKAFWAKVAGKEEQIGCDAVSRMLTVYPQTKTYFSHWKDLSPGSAPVKKHGAAVMAAVTDAVSKIDDLTG ALLSLSELHAFTLRVDPANFQVLSHNLLVVLSTMFPDDFTPEVHVAMDKFLAAVALALSEKYR

>Oryzias_latipes_LA_Hba2

MLSKKEKQLIREIWERLTPVAEDIGADALLRMFASYPGTKTYFSHLDIGPGSAHLSSHGKKIVLAIAEGAKDISQLTVTLAPLQTLH AYQLRIDPTNFKLLSYCLLVTLACYMGEGFTPEAHAAIDKFLSAFSAVLAEKYR

>Oryzias_latipes_MN_Hba1

MSLTAKDKDAVRTFWAKISGKADDVGTDALSRMLKVYPQTKTYFSHWTDLSPGSASVKKHGKTVIGGVADAVAKIDALSSGLL SLSELHAFTLRVDPANFKILGHCILVVMAMMFPNDFTPEVHVAMDKFLAALALALSEKYR

>Oryzias_latipes_MN_Hba2

MSLSAKDKAAVKAFWAKVSGQADAIGSDALSRMLVVYPQTKTYFAHWKDLSPGSAPVKKHGKTVMGGIADAVGKIDDISSGL LNLSELHAFTLRVDPTNFKILSHNILVVMAIMFPQDFTPEVHVALDKFLAAVSLALSEKYR 
>Oryzias_latipes_MN_Hba3

MSLTAKDKEVVKAFWTKLAPKADDVGADALSRMLVVYPQTKTYFAHWKDLSPGSAPVKKHGKTIMGGVADAVGKIDDLTAG LLDLSELHAFTLRVDPANFKILSHNILVVLAIVFPNEFTPEVHVALDKFLGALSRALAEKYR

>Oryzias_latipes_MN_Hba4

MTSLSAKDKDVVKAFWAKISSKATDIGADALGRMLVVYPQTKTYFAHWKDLSPGSAPVKKHGQTVMGGVAEAVGKIDNLTA GLLNLSELHAFTLRVDPANFKILSHNILVVLAIMFPNDFTPEVHVAMDKFLAALALALAEKYR

>Oryzias_latipes_MN_Hba5

MTSLSAKDKDVVKAFWAKISSKATDIGADALGRMLVVYPQTKTYFAHWKDLSPGSAPVKKHGQTVMGGVAEAVGKIDNLTA GLLNLSELHAFTLRVDPANFKILSHNILVVLAIMFPNDFTPEVHVAMDKFLAALALALAEKYR

>Salmon_salar_chr3_MN_HbaO

MSLSAKDKANVKAIWGKILPKSDEIGEQALSRMLVVYPQTKAYFSHWASVAPGSAPVKKHGITIMNQIDECVGNLDDLFGFLTK LSELHATKLRVDPTNFKILAHNLIVVVAAYFPAEFTPEIHLSVDKFLQQLALALAEKYR

>Salmon_salar_chr3_MN_Hba1

MSLTAKDKSVVKAFWGKISGKADVLGAEALGRMLTAYPQTKTYFSHWADLSPGSAPVKKHGGVIMGAIGNAVGLMDDLVGG MSGLSDLHAFKLRVDPGNFKILSHNILVTLAIHFPADFTPEVHIAVDKFLAALSAALADKYR

>Salmon_salar_chr3_MN_Hba2

MSLTAKDKQIVTAFFGKVAGKAEDIGNEALSRTLVVYPQTKTYFSHWTDLSPGSAPVKKHGLTVMGGVLDAVTKIDDLASGLLT LSELHAFTLRVDPANFKIINHNILVVLAMMFPDDFTPEVHLSVDKFLAKLALALSEKYR

>Salmon_salar_chr3_MN_Hba3

MSLTAKDKSVVKAFWGKISGKADVLGAEALGRMLTAYPQTKTYFSHWADLSPGSAPVKKHGGVIMGAIGNAVGLMDDLVGG MSGLSDLHAFKLRVDPGNFKILSHNILVTLAIHFPADFTPEVHIAVDKFLAALSAALADKYR

>Salmon_salar_chr3_MN_Hba4

MSLSAKDKANVKAIWGKILPKSDEIGEQALSRMLVVYPQTKAYFSHWASVAPGSAPVKKHGITIMNQIDECVGNLDDLFGFLTK LSELHATKLRVDPTNFKILAHNLIVVVAAYFPAEFTPEIHLSVDKFLQQLALALAEKYR

>Salmon_salar_chr3_MN_Hba5

MSLTAKDKSVVKAFWGKISGKADVLGAEALGRMLTAYPQTKTYFSHWADLSPGSAPVKKHGSTIMGAIGNAVGVIDDLVGGL SALSDLHAFKLRVDPGNFKILSHNILVTLAIHFPADFTPEVHIAVDKFLAALSAALADKYR

>Salmon_salar_chr3_MN_Hba6

MSLTAKDKKMVRAFWAKVSGKAEDIGCDALSRTLVVYPQTKTYFSHWKDLSPGSAPVRKHGGTIMGGISLAVASIDDISAGLLA LSELHAFKLRVDPANFKILSHNILVVLAILFPNDFNPEAHVAMDKFLAAVGRALSEKYR

>Salmon_salar_chr6_MN_HbaO

MSLTAKDKKIVKAFFGKVAGKAEDIGNEALSRTLVVYPQTKTYFSHWTDLRPGSAPVKKHGLTVMGGVLDAVTKIDDLTGGLLA LSELHAFTLRVDPANFKIINHNILVVLAMMFPDDFTPEVHVSVDKFLAKLALALSEKYR

>Salmon_salar_chr6_MN_Hba1 
MSLTTKDKKMVKAFWAKVSGKAEDIGCDALSRMLVVYPQTKTYFSHWKDLSPGSAPVRKHGGTIMGGISLAVASIDDISAGLL ALSELHAFQLRVDPANFKILSHNILVVLAVLFPNEFNPEAHVAMDKFLAAVGRALSEKYR

>Salmon_salar_chr6_MN_Hba2

MSLSAKDKANVKAIWGKILPKTDEIGEQALSRMLVVYPQTKTYFSHWTSVAPGSDPVKKHGITIMNQIDDCVGHLDDLFGFLTK LSELHATTLRVDPNNFKILAHNLIVVFAAYFPAEFTPEIHLSVDKFLQQVALALAEKYR

>Salmon_salar_chr6_MN_Hba3

MSLTARDKSVVNAFWGKIKGKADVVGAEALGRMLTAYPQTKTYFSHWADLSPGSAPVKKHGAVIMGAIGNAVGLMDNLVG GLSALSDLHAFKLRVDPGNFKILSHNILVTLAIHFPADFTPEVHIAVDKFLAALSAALADKYR

>Salmon_salar_chr6_MN_Hba4

MSLSAKDKANVKAIWGKILPKSDEIGEQALSRMLVVYPQTKAYFSHWASVAPGSAPVKKHGITIMNQIDECVGNLDDLFGFLTK LSELHATKLRVDPTNFKILAHNLIVVVAAYFPAEFTPEIHLSVDKFLQQLALALAEKYR

>Salmon_salar_chr6_MN_Hba5

MSLTAKDKSVVKAFWGKISGKADVVGAEALGRMLTAYPQTKTYFSHWADLSPGSAPVKKHGSTIMGAIGNAVGLIDDLVGGL SALSDLHAFKLRVDPGNFKILSHNILVTLAIHFPADFTPEVHIAVDKFLAALSAALADKYR

>Sparus_aurata_Hba1

MSLSDTDKARVRALWAKVQTKAPEIGGEALGRMLVAYPQTKAYFSHWGTDLNVNHPSVQKHGAVIMTGIARGVKYIDDLTN GLSALSELHAFKLRVDPGNFKILAHNLIVVIASYFPADFTPEAHVSFDKFLMRLAFALSERYR

>Sparus_aurata_Hba2

MSLSGRDKTIVKSFWDKIGSRSADIGAEALGRMLRVFPQTKTYFAHWSDLGPDSPQVRKHGSVIMAAVGEAIGKIDDLTGALSS LSELHAFKLRVDPANFRMLAHNIMLVIAMYYPGDFTAEVHLSFDKFLQNLALALAERCR

>Gadus_morhua_LA_Hba2

MSLSSKQKATVKDFFSKMSTRSDDIGAEALSRLVAVYPQTKSYFSHWKDASPGSAPVRKHGITIMGGVYDAVGKIDDLKGGLLS LSELHAFMLRVDPVNFKLLAHCMLVCMSMIFPEEFTPQVHVAVDKFLAQLALALAEKYR

>Takifugu_rubripes_LA_Hba1

MISQKEKRLLAEVWKSLIPAAEDIGSDSLLRMFTAFPGSKTYFSHLDISPRSPHMLSHGRKIVLAIAEGAQDISQLAVNLAPLQILH AYQLRIDPTNFKLLTHCLLVALACHLGDDFTPEAHAAIDKYLSAFAAVLAEKYR

>Takifugu_rubripes_LA_Hba2

MSLSRTDKEAVKAIWAKMSKSIDVIGAEAFGRMLIAYPQTKIYFSEWSDLRPASGPVKAHGKKVMGGIATAVASIDDLTCGLREL SERHAFTLKVDPANFRLLAHCILVVTAIMFPKDFTPEIHVSFDKFLAGVALALSDKYR

>Takifugu_rubripes_MN_Hba1

MSLSADDKKVVKAFWGKVSTKADAIGADALGRLLLVYPQTKTYFSHWKELTPNSNDVKIHGARIMTGVTEAIGKMDNLTVGLL NLSELHAFTLRVDPANFKLFAHCILVVMANMFPVDFTPEVHMAMDKFLAAVARGLSEKYR

>Takifugu_rubripes_MN_Hba2

MTSLSAKDKDTVRLFWGKLAPKREEVGANALCRLLSVYPQTKTYFAHWKDQSPTSASAKKHGITIMNAIGDSVSKIDDLKGGLF NLSELHAFTLRVDPANFKLLAHCIMVEIGIMYPTEFTPEVHVAVDKFLASVALALAEKYR 
>Tetraodon_nigroviridis_LA_Hba2

MSLTKTDKAAVKALWNKLSKSVDVIGAEAFGRMLLVYPQTKIYFSKWGDISFGSSQVKNHGKIVMGGIATAVANIDDLTSGLQK LSEVHAFDLKVDPANFKILGQCVVVVTAMLFPKDFTPEVHVSFDKFLAAVALALSEKYR

>Tetraodon_nigroviridis_MN_Hba1

MSLNANDKKVITTFWGKASAQADAIGSDALGRLLLAYPQTKTYFSHWKDLSPGSSDVKRHGALIMGGVTEAVTKIDNLSTGLLE LSELHAFTLRVDPANFKLFAHAIIVAIAIRFPGDFTPEVHMAMDKFFAAVARAMSEKYR

>Tetraodon_nigroviridis_MN_Hba2

MTSLSTKDKETVRAFWAKVASNREEIGASALCRLLSVYPQTKTYFSHWKDQSPNSASAKKHGITIMNAVGDAVSKIDDLKTGLF NLSELHAFTLRVDPANFKLLAQCMMVVIAIMYPADFTPEVHVAMDKFLASLALALSEKYR

>Tetraodon_nigroviridis_LA_Hba1

MITEKEKELLRKVWNSLIPVAEDIGSDSLLRLFTTVPGSKTYFSHLDISPRSPHMLSHGRKIVLAIAEGAQDISQLAVSLAPLQTLHA YQLRIDPTNFKLLTHCLLVSLACHMGDDFTPQAHAATDKYLSAFAAVLSEKYR

>Xiphophorus_maculatus_MN_Hba1

MSLSAKDKATVKTFWAKISSSGEEIGTDALSRMLVVYPQTKTYFSHWKDLSPGSAPVVKHGATVMAGVGDAVAKIDDLTSGLL SLSELHAFTLRVDPSNFQILSHNILVVLAIMFPTDFTPEVHVAMDKFLAAVARALSEKYR

>Trematomus_newnesi_Cathodic_HbA

AXSLSDKDKAAVRALWSKIGKSSDAIGNDALSRMIVVYPQTKIYFSHWPDVTPGSPNIKAHGKKVMGGIALAVSKIDDLKTGLM ELSEQHAYKLRVDPSNFKILNHCILVVISTMFPKEFTPEAHVSLDKFLSGVALALAERYR

>Xiphophorus_maculatus_MN_Hba2

MNLTAKDKDTVRTFWAKISTNAEDIGTDALSSMLVVYPQTKTYFSHWKDLSPGSAPVKKHGATVMAGVADAVAKIDDLRAGL LSLSELHAFTLRVDPANFQVLSHNILVVLATSFPNDFTPEVHVAMDKFLSAVALALSEKYR

>Xiphophorus_maculatus_MN_Hba3

MSLTAKDKETVKAFWAKVAPKAEDIGQDALSRMLAVYPQTKTYFSHWKDMSAGSAPVKKHGATVMGGVADAVTKIDDLTSG LLSLSELHAFTLRVDPANFQILAHNILVVFAIKFPTDFTPEVHVSVDKFLAALARALSEKYR

>Xiphophorus_maculatus_MN_Hba4

MSLSDKDKSRVKALWAKAEGKAGELGGEALGRMLVAYPQTKTYFSHWGDLSPQSPKVKKHGATIMGALGKAVKGIDDLPGTL AALSELHAFKLRVDPANFQILGHSIVVVLAMYFPGDFTPEVHLSVEKFLQNVALALSEKYR

>Xiphophorus_maculatus_MN_Hba5

MTSLTAKDKDTVKAFWGKISPKAGAIGADALGRMLVVYPQTKTYFSHWKDMSPGSGPVKKHGATVMAGVADAVAKIDDLTA GLLTLSELHAFKLRVDPANQILAHNLLLVLAISFPNDFTPEVHVAMDKFLSAVALALSEKYR

>Xiphophorus_maculatus_LA_Hba2

MLSKKEKKLIKEMWERLTPVAEDIGSEALLRMFASYPGTKTYFSHLDISPGSAHLSSHGKKIVLAIAEGAKDISQLTVTLAPLQTLH AYQLRIDPTNFKLLSHCMLVTLACYLGSEFTPVAHAAMDKFLSAFAAVLSEKYR 


\section{$\underline{\text { Hemoglobin beta sequences }}$}

>Anguilla_anguilla_Anodic_Hbb

VEWTEDERTAIKSKWLKINIEEIGPQAMRRLLIVCPWTQRHFANFGNLSTAAAIMNNDKVAKHGTTVMGGLDRAIQNMDDIK NAYRQLSVMHSEKLHVDPDNFRLLAEHITLCMAAKFGPTEFTADVQEAWQKFLMAVTSALARQYH

>Danio_rerio_LA_Hbb1

MVAWTAEERAFIQDIFSKLNYEEAGPKALQRALIVYPWTQRYFGSFGNLYNAEAIINNPKVAAHGTVVLHGLDRAMKNMDDIK NTYAELSVLHSEKLHVDPDNFRLLADCLTIVIASTMGAAFTADMQAAWQKFLAVVVSALQRQYH

>Danio_rerio_LA_Hbb2

MVVWTAEERAAIQNIFAKLDFESVGLETLTRCLVVYPWTQRYFGGFGNLYNTEAIMANPKVKAHGVVVLKGLEKALNNMDNI KSTYASLSELHSEKLQVDPGNFRLLADCLTVVIATRMRSEFTPDIQAAWQKFLSVVVSALRRQYL

>Danio_rerio_MN_Hbb1

MVQWSDSERKTIASVWSKINVDEIGPQTLARVLVVYPWTQRYFGAFGDLSCASAIMGNPKVSEHGKTVLKALEKAVKNVDDIK TTYAKLSQLHCEKLNVDPDNFKLLADCLSIVIATNFGPAFNPSVQSTWQKLLSVVVAALTSRYF

>Danio_rerio_MN_Hbb2

MVEWTDAERTAILGLWGKLNIDEIGPQALSRCLIVYPWTQRYFATFGNLSSPAAIMGNPKVAAHGRTVMGGLERAIKNMDNV KNTYAALSVMHSEKLHVDPDNFRLLADCITVCAAMKFGQAGFNADVQEAWQKFLAVVVSALCRQYH

>Danio_rerio_MN_Hbb3

MVEWTDAERTAILGLWGKLNIDEIGPQALSRCLIVYPWTQRYFATFGNLSSPAAIMGNPKVAAHGRTVMGGLERAIKNMDNV KNTYAALSVMHSEKLHVDPDNFRLLADCITVCAAMKFGQAGFNADVQEAWQKFLAVVVSALCRQYH

>Danio_rerio_MN_Hbb4

MVVWTDFEKATIQDIFAKADYDVIGPQALARCLIVYPWTQRYFAKFGNLYNAAAILGNPMVAAHGKTVLKGLELAVKNMDNIK ATYADLSVLHSEKLHVDPDNFRLLADCLTIVVAAQMGAGFTPEVQAAFQKFIAVAVSALGRQYH

>Danio_rerio_MN_Hbb5

MVVWTDFEKATIQDIFAKADYDVIGPQALARCLIVYPWTQRYFAKFGNLYNAAAILGNPMVAAHGKTVLKGLELAVKNMDNIK ATYADLSVLHSEKLHVDPDNFRLLADCLTIVVAAQMGAGFTPEVQAAFQKFIAVAVSALGRQYH

>Danio_rerio_MN_Hbb6

MVVWTDFEKATIQDIFAKADYDVIGPQALARCLIVYPWTQRYFAKFGNLYNAAAILGNPMVAAHGKTVLKGLELAVKNMDNIK ATYADLSVLHSEKLHVDPDNFRLLADCLTIVVAAQMGAGFTPEVQAAFQKFIAVAVSALGRQYH

>Dasyatis_akajei_Hbb

MVKLSEDQEHYIKGVWKDVDHKQITAKALERVFVVYPWTTRLFSKLQGLFSANDIGVQQHADKVQRALGEAIDDLKKVEINFQ NLSGKHQEIGVDTQNFKLLGQTFMVELALHYKKTFRPKEHAAAYKFFRLVAEALSSNYH

>Dicentrarchus_labrax_LA_Hbb1

MVEWTEQERGIITSIFSSLDYEDIGPKALCRCLIVYPWTQRYFGAFGNLYNAEAIKSNPNIAAHGVTVLHGLDRAVKNMDNIKAT YAELSVLHSEKLHVDPDNFKLLSDCLTIVIAAKLGNGFTPETQAAWQKFLAVVVSALGRQYH 
>Dicentrarchus_labrax_MN_Hbb1

MVKWTDAERAAITSWWGKIDVNEIGPQALTRLLIVYPWTQRHFATFGNLSTSAAILGNPLVAEHGKTVMGGLDRAVKNMDDI KNVYTKLSVKHSEKIHVDPDNFRIFAQIISVCVAAKFGRQFTPDVQEAWQKFLDVVVSALSRQYH

>Dicentrarchus_labrax_MN_Hbb2

MVEWTDFERATIQDIFSKMDYEVVGPAALSRCLVVYPWTQRYFGNFGNLYNAAAIIANPMVAAHGKVVLHGLDRAVKNMDD IKGTYAELSVLHSEKLHVDPDNFRLLADCLTIVVAAKLGKAFTGDVQAAFQKFLAVVVSSLGRQYH

>Dicentrarchus_labrax_MN_Hbb3

MVEWTDKERSTVSAIWEKIDIDVIGPEALARVLIVYPWTERYFGTFGDIFSATAVLGNAKVAAHGKVVLKALDSAVKNMDNIKA TYADLSRLHYEKLSVDPDNFKLLADCITIAVACKLKSALNPQAQATWQKFLSVVVDAMSSQYF

>Dicentrarchus_labrax_MN_Hbb4

MVEWSDFERATIQDIFSKMEYEVVGPAALSRCLVVYPWTQRYFGNFGNLYNAAAIIANPMVAKHGTTILHGLDRAVKNMDDI KATYAELSVLHSEKLHVDPDNFKLLSDCLTIVVAAKLGKAFTGDVQAAFQKFLAVVVSSLGMQYH

>Dicentrarchus_labrax_MN_Hbb5

MVEWSDFERATIQDIFSKMEYEVVGPAALSRCLVVYPWTQRYFGNFGNLYNAAAIIANPLVAKHGTTILHGLDRAVKNMDDIK ATYAELSVLHSEKLHVDPDNFKLLSDCLTIVVAAKLGKAFTGDVQAAFQKFLAVVVSSLGMQYH

>Dicentrarchus_labrax_MN_Hbb6

MVEWSDFERATIQDIFSKMEYEVVGPAALSRCLVVYPWTQRYFGNFGNLYNAAAIIANPLVAKHGTTILHGLDRAVKNMDDIK ATYAELSVLHSEKLHVDPDNFKLLSDCLTIVVAAKLGKDFTGDVQAAFQKFLAVVVSSLGRQYH

>Dicentrarchus_labrax_Hbb_LP

MVQWSDAERAAITSWWEKIDVNEIGPQALTRLLIVYPWTQRHFATFGNLSTNAAILGNPLVAEHGKTVMGGLDRAVKNMDD IKNVYTKLSVKHSEKIHVDPDNLRIFAQIISVCVAAKFGRQFTPDVQEAWQKFLAVVV

>Sparus_aurata_Hbb1

MVQWSDAERAAIVSLWGKIDVGEIGPQALSRLLIVYPWTQRHFSGFGDISTNAAIVANDKVAAHGKTVMGGLDRAVKNLDDI KNAYTKLSQKHSEQIHVDPDNFRLLAECISVCVGAKFGPKVFNADAQEAWQKFLAVVVAALGKQYH

>Dipturus_batis_Hbb

MVKITDKEAAYITDIWSKLDKKVTTAHALERVFTVYPWTTRLFKSFNGHFKAGDSGVQGHAEKVVGALDTAVLHLHDIDAGYKK LSEKHQLIGVDTQNFKLLGQAFLVELAILFKEGFTPELHEAAYKFFLAVAGGLSSQYH

>Gadus_morhua_LA_Hbb2

MVEWTDEERTIINDIFSTLDYEEIGRKSLCRCLIVYPWTQRYFGAFGNLYNAETIMANPLIAAHGTKILHGLDRALKNMDDIKNTY AELSLLHSDKLHVDPDNFRLLADCLTVVIAAKMGTKFTVETQVAWQKFLSVVVSALGRQYH

>Gadus_morhua_LA_Hbb3

MVEWTDSERAIINGIFSNLDYEEIGRKSLCRCLIVYPWTQRYFGGFGNLYNAETILCNPLIAAHGTKILHGLDRALKNMDDIKNTY AELSLLHSDKLHVDPDNFRLLADCLTVVIAAKMGPAFTVDTQVAWQKFLSVVVSALGRQYH

>Gadus_morhua_LA_Hbb4 
MVEWTDSERAIITSIFSNLDYEEIGRKSLCRCLIVYPWTQRYFGGFGNLYNAETILCNPLIAAHGTKILHGLDRALKNMDDIKNTYA ELSLLHSDKLHVDPDNFRLLADCLTVVIAAKMGPAFTVDTQVAWQKFLSVVVSALGRQYH

>Gadus_morhua_MN_Hbb1

MVEWTAAERRHVEAVWSKIDIDVCGPLALQRCLIVYPWTQRYFGSFGDLSTDAAIVGNPKVAAHGVVALTGLRTALDHMDEI KSTYAALSVLHSEKLHVDPDNFRLLCECLTIVVAGKMGKKLSPEMQAAWQKYLCAVVSALGRQYH

>Gadus_morhua_MN_Hbb5

MVEWTEFERDTIKDIFSKIDYDVVGPAALTRCLVVYPWTRRYFGNFGALYNAEAIMGNEMVANHGKKVLHGLDRAVKNMDHI KESYCELSQLHSDQFHVDPDNFRLLADCLAIAIATQWGSAFTPDIQAAFQKFLSVVVFSLGSQYH

>Gasterosteus_aculeatus_MN_Hbb1

MVVWTDFERSTIQDIFSKMDYEVVGPAALTRCLIVYPWTQRYFGNFGNLYNAAAIMGNPMVAKHGTTILHGLDRGVKNMDD LKATYAELSVLHSEKLHVDPDNFKLLSDCLTIVVAAKLGKAFTGEVQAAFQKFLAVVVSSLGRQYH

>Gasterosteus_aculeatus_MN_Hbb2

MVVWTDFERSTIQDIFSKMDYEVVGPAALTRCLIVYPWTQRYFGNFGNLYNAAAIMGNPMVAKHGTTILHGLDRGVKNMDD LKTTYAELSVLHSEKLHVDPDNFKLLSDCLTIVVAAQLGKAFTAEVQAAFQKFLAVVVSSLGRQYH

>Gasterosteus_aculeatus_MN_Hbb3

MVAWTEFERATIQDIFSKIDYDVVGPAALSRCLVVYPWTQRYFGNFGNLYNAAAIMGNPLVAKHGTTILNGLERAVKDMDNIK ATYAELSVLHSEKLHVDPDNFKLLSDCLTIVVAARFGEAFTAEVQAAFQKFLAVVVSSLGRQYH

>Gasterosteus_aculeatus_MN_Hbb4

MVAWTEFERATIKDIFSKIDYDVVGPAALCRCLVVYPWTQRYFGNFGNLYNAAAIMGNPLVAKHGTTILNGLERAVKDMDNIK TTYAELSVLHSEKLHVDPDNFKLLSDCLTIVVAARFGEAFTAEVQAAFQKFLAVVVSSLGRQYH

>Gasterosteus_aculeatus_MN_Hbb5

MVAWTEFERATIKDIFSKIDYDVVGPAALCRCLVVYPWTQRYFGNFGNLYNAAAIMGNPLVAKHGTTILNGLERAVKDMDNIK TTYAELSVLHSEKLHVDPDNFKLLSDCLTIVVAAQFGKAFTAEVQAAFQKFLAVVVSSLGRQYH

>Gasterosteus_aculeatus_MN_Hbb6

MVAWTEFERATIKDIFSKIDYDVVGPAALCRCLVVYPWTQRYFGNFGNLYNAAAIMGNPLVAKHGTTILYGLERAVKDMDNIK TTYAELSVLHSEKLHVDPDNFKLLSDCLTIVVAARFGEAFTAEVQAAFQKFLAVVVSSLGRQYH

>Homo_sapiens_Hbb

MVHLTPEEKSAVTALWGKVNVDEVGGEALGRLLVVYPWTQRFFESFGDLSTPDAVMGNPKVKAHGKKVLGAFSDGLAHLDN LKGTFATLSELHCDKLHVDPENFRLLGNVLVCVLAHHFGKEFTPPVQAAYQKVVAGVANALAHKYH

>Ictalurus_punctatus_MN_Hbb1

MVSWSQSERSAIADLWDKINVDEIGPQSLARVLIVYPWTQRYFGKFGNLNSVAAILGNPKVTAHGKVVLNALDNAVKNLDNIK ETYSKLSQLHCEILSVDPDNFRLFADCLTIVIANKFGAAFTPEIQATWQKFLAVVVAAMSSRYF

>Ictalurus_punctatus_MN_Hbb2

MVHWTDAERHIIADLWGKINHDEIGGQALARLLIVYPWTQRYFSSFGNLSNAAAIIGNPKVAAHGKVVLGGLTKAVQNLDNVK GIYTQLSTLHSEKLHVDPSNFTLLGDTFTVTLAANFGPSVFTPEVHETWQKFLNVVVAALGKQYH 
>Ictalurus_punctatus_MN_Hbb3

MVHWTDAERHIIADLWGKINHDEIGGQALARLLIVYPWTQRYFSSFGNLSNAAAIIGNPKVAAHGKVVLGGLTKAVQNLDNVK GIYTQLSTLHSEKLHVDPSNFTLLGDTFTVTLAANFGPSVFTPEVHETWQKFLNVVVAALGKQYH

>Ictalurus_punctatus_MN_Hbb4

MVVWTDFERATIQDVFSKIDYESVGHQALSRCLVVYPWTQRYFGSFGNLYNAAAIIGNPKVAAHGLVVVRGLEKAAKNMDNIK AIYADLSVLHSEKLHVDPDNFKLLADCITIVIASVLGASFTAEVQAALQKFLAVVVSALGKQYQ

>Ictalurus_punctatus_MN_Hbb5

MVVWTDFERATIQDLFSKIDYESVGHQALSRCLVVYPWTQRYFGSFGNLYNAAAIIGNPKVAAHGLVVVRGLEKAAKNMDNIK AIYADLSVLHSEKLHVDPDNFKLLADCITIVIASVLGASFTAEVQAALHKFLAVVVSALGKQYQ

>Ictalurus_punctatus_MN_Hbb6

MVQWTDFERTTIQDIFSKIDYESVGHQALTRCLVVYPWTQRYFSKFGNLYNAAAILGNPKVAAHGLTVMRGLEKAVKNMDNIK STYADLSVLHSEQLHVDPQNFRLLADCITIVVASVLGSSFTAEVQAALQKFLAVIVSALGKQYY

>Lepisosteus_oculatus_Hbb1

MVHFTDAEAKAIKTVWGKVNVDTVGPQALARLLIVYPWTQRYFGAFGNLTNAAAILGNAKVAHHGKVVLGALDKAVQDPEHI TANYASLSELHSTKLHVDPDNFRVLGDCITIVLAAQLGTGFTVELNAAFQKFLDVVIAALRKQYH

>Lepisosteus_oculatus_Hbb2

MVHFTDAEAKAIKTVWGKVNVDIVGPQALARLLIVYPWTQRYFGAFGNLTNAAAILGNAKVAHHGKVVLGALDKAVHDLEHI VANYASLSELHSTKLHVDPDNFRVLGDCITIVLAAQLGTGFTVELNAAFQKFLDVVIAALRKOYH

>Lepisosteus_oculatus_Hbb4

MVHWTAAERQAIASVWEKISLESVGSQALARLLIVYPWTQRYFSSFGNLSSAAAILGNPKVKHHGKTVLTALGQAVKNMDNIK ATFSKLSELHSEKLHVDPGNFRMLGETLIIVLAAELGAEFTPELQAAWQKLLSVVSSALSRQYY

>Lepisosteus_oculatus_Hbb5

MVHWSDEERKAITSIWGKVDVESAGHQALVRLLIVYPWTQRYFSSFGNLSSAAAILGNPKVKHHGKTVLTALGDAVKNMDNIK ATFSKLSELHSEKLHVDPENFRLLGETLVIVLAGQFGAEFTPAVQAAWQKLLAVSISALSKQYH

>Mustelus_griseus_Hbb

MVHWTQEERDEISKTFQGTDMKTVVTQALDRMFKVYPWTNRYFQKRTDFRSSIHAGIVVGALQDAVKHMDDVKTLFKDLSK KHADDLHVDPGSFHLLTDCIIVELAYLRKDCFTPHIQGIWDKFFEVVIDAISKQYH

>Oryzias_latipes_LA_Hbb1

MVEWTEQERSIITNIFGNLDYEDVGSKALSRCLIVYPWTQRYFASFGNLYNAEAIKTNPNIAAHGTKVLHGLDRAVKNMDNIKA TYAELSVLHSEKLHVDPDNFKLLADCLTIVIAAKLGSAFSPEIQATFQKFLAVVVSALGRQYH

>Oryzias_latipes_MN_Hbb1

MVKWSDFERATIQDIFSKIDKDVVGQAALSRCLIVYPWTQRYFGSFGNLYNAAAITSNPKVAAHGKVVIGGLEKALQNMDNIK QAYTDLSVLHSEKLQVDPDNFKLLADCLTIVVASNLGISFTPEVQAAFQKFLDVVVAALRKQYY

>Oryzias_latipes_MN_Hbb2 
MVTWTDFERATIQDIFSKIDYDIVGPDALSRCLIVYPWTQRYFGSFGNLYNAAAIASNPKVAAHGKVVLGGLEKALKNMDDIKQ AYADLSVLHSEKLQVDPDNFKLLADCLTIVVASQLGKDFTPEVHAAFAKFLAVVVAALRKQYH

>Oryzias_latipes_MN_Hbb3

MVKWTEEERCTIREVWEKVDVDEIGPQILTRVHIVYPWRETYFGTFGDIFTNTSILNNPKVAHHGKVVLRSIDKAVRIMDRIQET HAALSRLHYECVDPDNFKLLGDCITISIACKLKEALNPQVQAVWQKFLCAVVEAMNSQNK

>Oryzias_latipes_MN_Hbb4

MVEWTDFERATIQDIFSRIDKDVVGPAALSRCLIVYPWTQRYFGSFGNLYNAAAITSNPKVAAHGKVVLSGLELAVKNMDDIKQ TYADLSVLHSEKLHVDPDNFKLLADCLTIVVAAQMGPDFTCDVQAAFQKFLAVVVSSLRRQYH

>Oncorhynchus_mykiss_HbbE

MVQWTDFERATIQSVFEKMDYDDVGPAALSRCLVVYPWTQRYFGNFGNLYNAAAIQGNPMVAAHGKTVLRGLDRAVKNM DDIKATYAELSVLHSEKLRVDPDNFRLLADCLTIVVAARMGADFTADVQGAFQKFLAVVVSSLGRQYH

>Oncorhynchus_mykiss_Hbb2

MVVWTDEERAAISDIFSKLDYDDVGPKCLSRCLIVYPWTQRYFGAFGNLYNAEAIMNNPLIAKHGITVLRGLERALKNMDDIKN TYAELSILHSEKLRVDPDNFKLLSDCLTIVIAAKMGTAFTPEYQASFQKFLSVVVSALGRQYH

>Oreochromis_niloticus_MN_Hbb1

MVEWTDAERKAIASLWGKIDVGEIGPQALARLLIVYPWAQRYFKAFGDLSTNAAIMGNPKVAQHGKTVMGGLENAVKNLDNI KQTYAKLSVMHSEKLHVDPDNFRXXXXXXXXXXXXXXXXXXXXXXXQEAWOKFLAVVVSALGRQYH

>Oreochromis_niloticus_MN_Hbb3

MVVWTDFERTTIQDIFSKIDYAVVGQAAFSRCLTVYPWTQRYFGQFGNLYNAAAIASNPKVAAHGKVIMEALEKAVKDMDNIK ATYAELSALHSEKLQVDPDNFMLLGDCLTIVVASQLGKNFTAEVHAAFQKFLAVVVSSLRRQYY

>Oreochromis_niloticus_MN_Hbb4

MVVWTDFERTTIQDIFSKIDYAVVGQAAFSRCLIVYPWTQRYFGGFGNLYNAAAITTNPKVAAHGKVIMEALEKAVKDMDNIK ATYAEXXXXXXXSEGHGQHQGHLLGDCLTIVVASQLGKNFTAEVHAAFQKFLAVVVSSLRRQYY

>Oreochromis_niloticus_MN_Hbb5

MVKWTELERSTVKAIWEKVDIDEIGPQIWARVLIVYPWTERYFGSFGDLFTITAILKNDKVAAHGKVVLKALDKAVNNMDNMK RTYADLSQLHFQKLEVDPDSFRLLADCITITIACKLKSALSPQSOAIWQKFLYAVVDAMSSOYR

>Oreochromis_niloticus_MN_Hbb6

MVEWTDAERSAITSLWGKIDVGEIGPQALTRLLIVYPWAQRYFQSFGDLSTNAAIMGNPKVAQHGRTVMGGLENAVKNLDNI KNTYANLSHMHSEKLHVDPDNFRVLAECISLCVAAKFGPSVFTADAQEAWQKFLAVVVSALGRQYH

>Oreochromis_niloticus_MN_Hbb8

MVAWTDFERATIKDIFSKIDYEVVGPAAISRCLIVYPWTQRYFAGFGNLYNAAAITSNPKVAAHGKVVMQGLEKAVKNMDNIK ATFTELSTLHSEKLQVDPDNFMLLGDCLAIVVASQLGKDFSPEVHAAFQKFLAVVVSSLRRQYY

>Oreochromis_niloticus_LA_Hbb1

MVEWSEKERSIITNIFSNLDYEDVGPKALVRCLVVYPWTQRYFASFGNLYNAEAISTNPKVAAHGIKVLHGLDRAVKNMDNIKA TYAELSVLHSEKLHVDPDNFKLLSDCLTIVVAGKLGSAFTPEVQATFQKFLAVVVSALGKQYH 
>Salmon_salar_chr3_MN_Hbb0

MVDWTDAEKSTISAVWGKVDINEVGPLALARVLIVYPWTQRYFGSFGDVSTPAAIMGNPKVAAHGKVVCGALDKAVKNMG NILATYKSLSETHANKLFVDPDNFRVLADVLTIVVAAKFGASFTPEIQATWQKFMKVVVAAMGSRYF

>Salmon_salar_chr3_MN_Hbb1

MVDWTDAERSAIVGLWGKISVDEIGPQALARLLIVSPWTQRHFSTFGNLSTPAAIMGNPAVAKHGKTVMHGLDRAVQNLDDI KNAYTALSVMHSEKLHVDPDNFRLLADCITVCVAAKLGPTVFSADIQEAFQKFLAVVVSALGRQYH

>Salmon_salar_chr3_MN_Hbb2

MVDWTDAEKSTISAVWGKVDINEVGPLALARVLIVYPWTQRYFGSFGDVSTPAAIMGNPKVAAHGKVVCGALDKAVKNMG NILATYKSLSETHANTLFVDPDNFRVLADVLTIVIAAKFGASFTPEIQATWQKFMKVVVAAMGSRYF

>Salmon_salar_chr3_MN_Hbb3

MVDWTDAEKSTISAVWGKVDINEVGPLALARVLIVYPWTQRYFGSFGDVSTPAAIMGNPKVAAHGKVVCGALDKAVKNMG NILATYKSLSETHANKLFVDPDNFRVLADVLTIVIAAKFGASFTPEIQATWQKFMKVVVAAMGSRYF

>Salmon_salar_chr3_MN_Hbb4

MVDWTDAERSAIVGLWGKISVDEIGPQALARLLIVSPWTQRHFSTFGNLSTPAAIMGNPAVAKHGKTVMHGLDRAVQNLDDI KNAYTALSVMHSEKLHVDPDNFRLLADCITVCVAAKLGPAVFSADIQEAFQKFLSVVVSALGRQYH

>Salmon_salar_chr3_MN_Hbb5

MVDWTDAEKSTISAVWGKVDINEVGPLALARVLIVYPWTQRYFGSFGDVSTPAAIMGNPKVAAHGKVVCGALDKAVKNMG NILATYKSLSETHANKLFVDPENFRVLADVLTIVVAAKFGASFTPEIQATWQKFMKVVVAAMGSRYF

>Salmon_salar_chr3_MN_Hbb6

MVQWTDFERATIQSVFEKMDYDDVGPAALSRCLVVYPWTQRYFGNFGNLYNAAAIQGNPMVAAHGKTVLRGLDRAVKNM DDIKATYAELSVLHSEKLHVDPDNFRLLADCLTIVVAARMGADFTADVQGAFQKFLAVVVSSLGRQYH

>Salmon_salar_chr3_MN_Hbb7

MVEWTDFERATIQSIFSKMDYDDVGPAALSRCLVVYPWTQRYFGNFGNLYNAAAIQGNPMVAAHGKTVLRGLDRAVKNMD DIKATYAELSVLHSEKLHVDPDNFRLLADCLTIVVAARMGADFTADVQGAFQKFLAVVVSSLGRQYH

>Salmon_salar_chr6_MN_HbbO

MVQWTDFERQTIQSIFGKMDYDDVGPAALSRCLIVYPWTQRYFGNFGNLSNAAAIQGNPKVAAHGKTVLQGLVRAVKNMD DIKATFTELSVLHSEKLRVDPDNFRLLADCLTIVVAARMGADFTADVQGAFQKFLAIVVCSLCRQYH

>Salmon_salar_chr6_MN_Hbb1

MVQWTDFERATIQSVFEKMDYDVVGPAALSRCLVVYPWTQRYFGNFGNLYNAAAIQGNPMVAAHGKTVLHGLDRAVKNM DDIKATYAELSVLHSEKLHVDPDNFRLLADCLTIVVAARMGADFTADVQGAFQKFLAVVVSSLGKQYH

>Salmon_salar_chr6_MN_Hbb2

MVDWTDAERSAILGLWGNISVDEIGPQALARLLIVFPWTQRHFSTFGNLSTPAAIMGNPAVAKHGKVVMHGLDRAVQNLDDI KNAYSALSVKHSEKLHVDPDNFRLLAECITVCVAAKLGPAVFSADIQEAFQKFLAVVVSALGRQYH

>Salmon_salar_chr6_MN_Hbb3 
MVDWTDAEKSTISAVWGKVDINEVGPLALARVLIVYPWTQRYFGSFGDVSTPAAIMGNPKVAAHGKVVCGALDKAVKNMG NILATYKSLSETHANKLFVDPDNFRVLADVLTIVIAAKFGASFTPEIQATWQKFMKVVVAAMGSRYF

>Salmon_salar_chr6_MN_Hbb4

MVDWTDAERSAILGLWGKISVDEIGPQALARLLIVSPWTQRHFSTFGNLSTPAAIMGNPAVAKHGKTVMHGLDRAVQNLDDI KNAYSALSVMHSEKLHVDPDNFRLLADCITVCVAAKLGPAVFSADIQEAFQKFLAVVVSALGRQYH

>Salmon_salar_chr6_MN_Hbb5

MADWTDAEKSTISAVWGKVDINEVGPLALARVLIVYPWTQRYFGSFGDVSTPAAIMGNPKVAAHGKVVCGALDKAVKNMG NILATYKSLSETHANKLFVDPENFRVLADVLTIVIAAKFGASFTPEIQATWQKFMKVVVAAMGSRYF

$>$ Takifugu_rubripes_LA_Hbb1

MVEWTDQERTIISNIFSTLDYEDVGSKSLIRCLIVYPWTQRYFAGFGNLYNAEAIKNNPNIAKHGVTVLHGLDRAVKNMDNIKET YKELSELHSEKLHVDPDNFKLLSDCLTIVVATKMGSKFTPEIQATFQKFLAVVVSALGRQYH

>Tetraodon_nigroviridis_LA_Hbb1

MVVWTDQERAIIDNIFSNLDYEDVGSKALIRCLIVYPWTQRYFSSFGNLYNAEAIRNNPNVAKHGVTVLHGLDRALKNMDNIKE EYKKLSELHSEKLHVDPDNFKLLSDCLTVVIAGKLGSKFTPEYQAALQKFLAVVVSALGRQYH

>Trematomus_newnesi_Cathodic_Hbb

VEWTDFERATIKDIFSKLEYDVVGPATLARCLVVYPWTQRYFGKFGNLYNAAAIAQNAMVSKHGTTILNGLDRAVKNMDDITN TYAELSVLHSEKLHVDPDNFKLLADCLTIVVAARFGSAFTGEVQAAFQKFMAVVVSSLGKQYR

>Xiphophorus_maculatus_MN_Hbb1

MVKWSDFERAAIQDIFSKINYDVVGCAALSRCLIVYPWTQRYFGSFGNLYNAAAITSNPKVAAHGKVIMAGLEKAVKNMDDIK TTYKDLSVLHSEKLQVDPDNFNLLADCLTIVVAGQMGAAFTPEVHGAFQKFLAVVVASLRKQYH

>Xiphophorus_maculatus_MN_Hbb2

MVKWSDFERAAIQDIFSKINYDVVGCAALSRCLIVYPWTQRYFGGFGNLYNAAAITSNPKVAAHGKVIMAGLEKAVKNMDDIK TTYKDLSVLHSEKLQVDPDNFNLLADCLTIVVAGQMGAAFTPEAHGAFQKFLAVVVASLRKQYH

>Xiphophorus_maculatus_MN_Hbb4

MVEWTDAERTAISTLWSNIDVGEIGPQALSRLLVVFPWTQRYFPTFGDLSTPAAIAANPKVAQHGKTVMGGLETAVKNIDNIK NAYAKLSVMHSEKLHVDPDNFRLAECITVVVAAKFGPSVFTAGFQEAWQKFLAVVVSALGRQYH

>Xiphophorus_maculatus_Hbb1

MVEWTDEERKAITTLWGQIDVGEIGPQALTRLLIVYPWTQRHFGAFGNLSTYAAIVGNAKVAQHGKTVMSGLETAVKNMDNI KNAYAKLSVMHSDKLHVDPDNFRLAECISVVVAAKFGPTVFTASFOEAWQKFLAVVVSALGRQYH

>Xiphophorus_maculatus_LA_Hbb1

MVAWSDQERSIITSIFSNLDYNDVGPKALIRCLIVYPWTQRYFASFGNLYNADAIKTNPNIQAHGTKVLHGLDRAVKNMDDIKT TYAELSVLHSEKLHVDPDNFKLLADCLTIVIAAKMGSGFTPEIQATFQKFLAVVVSALGKQYH 
Supplementary file 4: Identity matrix based on the nucleotides (panel A) and amino acid (panel B) sequences of European sea bass $\mathrm{Hb} \alpha$ and $\mathrm{Hb} \beta$ genes.

\begin{tabular}{|c|c|c|c|c|c|c|c|}
\hline LA-Hba2 & 100.00 & & & & & & \\
\hline $\mathrm{MN}-H b \alpha 1$ & 52.35 & 100.00 & & $A$ & & & \\
\hline LA-Hb $\alpha 1$ & 53.99 & 65.74 & 100.00 & & & & \\
\hline $\mathrm{MN}-H b \alpha 2$ & 54.23 & 72.22 & 72.69 & 100.00 & & & \\
\hline $\mathrm{MN}-\mathrm{Hb} \alpha 4$ & 54.46 & 70.83 & 69.44 & 88.19 & 100.00 & & \\
\hline $\mathrm{MN}-\mathrm{Hb} \alpha 5$ & 54.46 & 71.30 & 70.14 & 87.73 & 98.39 & 100.00 & \\
\hline $\mathrm{MN}-\mathrm{Hb} \alpha 3$ & 54.23 & 71.30 & 69.91 & 87.96 & 98.62 & 99.77 & 100.00 \\
\hline $\mathrm{MN}-H b \beta 1$ & 100.00 & & & & & & \\
\hline LA- $H b \beta 1$ & 65.91 & 100.00 & & & & & \\
\hline $\mathrm{MN}-H b \beta 3$ & 61.94 & 67.72 & 100.00 & & & & \\
\hline $\mathrm{MN}-H b \beta 2$ & 65.54 & 76.75 & 65.99 & 100.00 & & & \\
\hline $\mathrm{MN}-\mathrm{Hb} \beta 6$ & 65.99 & 76.98 & 65.09 & 93.92 & 100.00 & & \\
\hline $\mathrm{MN}-\mathrm{Hb} \beta 4$ & 65.77 & 77.20 & 65.54 & 93.92 & 98.20 & 100.00 & \\
\hline $\mathrm{MN}-H b \beta 5$ & 65.77 & 76.98 & 65.32 & 93.92 & 98.65 & 99.55 & 100.00 \\
\hline
\end{tabular}

\begin{tabular}{|c|c|c|c|c|c|c|c|}
\hline LA-Hb $\alpha 2$ & 100.00 & & & \multirow{3}{*}{ B } & & & \\
\hline $\mathrm{MN}-H b \alpha 1$ & 39.01 & 100.00 & & & & & \\
\hline LA-Hb $\alpha 1$ & 48.23 & 60.84 & 100.00 & & & & \\
\hline $\mathrm{MN}-\mathrm{Hb} \alpha 2$ & 45.39 & 68.53 & 74.13 & 100.00 & & & \\
\hline $\mathrm{MN}-H b \alpha 3$ & 46.10 & 68.53 & 67.13 & 81.12 & 100.00 & & \\
\hline $\mathrm{MN}-\mathrm{Hb} \alpha 5$ & 46.10 & 68.53 & 67.13 & 81.12 & 100 & 100.00 & \\
\hline $\mathrm{MN}-\mathrm{Hb} \alpha 4$ & 46.10 & 69.93 & 67.83 & 81.82 & 97.92 & 97.92 & 100.00 \\
\hline
\end{tabular}

\begin{tabular}{|c|c|c|c|c|c|c|c|}
\hline $\mathrm{MN}-H b \beta 3$ & 100.00 & & & & & & \\
\hline $\mathrm{MN}-H b \beta 1$ & 54.42 & 100.00 & & & & & \\
\hline LA- $H b \beta 1$ & 60.54 & 61.22 & 100.00 & & & & \\
\hline $\mathrm{MN}-H b \beta 2$ & 61.90 & 63.95 & 77.55 & 100.00 & & & \\
\hline $\mathrm{MN}-\mathrm{Hb} \beta 6$ & 57.82 & 61.22 & 77.55 & 92.52 & 100.00 & & \\
\hline $\mathrm{MN}-\mathrm{Hb} \beta 4$ & 58.50 & 59.86 & 76.87 & 93.20 & 97.96 & 100.00 & \\
\hline $\mathrm{MN}-H b \beta 5$ & 58.50 & 60.54 & 76.87 & 92.52 & 98.64 & 99.32 & 100.00 \\
\hline
\end{tabular}


Supplementary file 5. Cq (Quantification cycle) values of $\mathrm{Hb} \alpha$ and $\mathrm{Hb} \beta$ genes at different developmental stages of European sea bass. ND means Not Detected.

\begin{tabular}{|c|c|c|c|c|c|c|c|c|c|}
\hline Gene & $12 \mathrm{hpf}$ & $60 \mathrm{hpf}$ & $5 \mathrm{dph}$ & $13 \mathrm{dph}$ & $19 \mathrm{dph}$ & $26 \mathrm{dph}$ & $33 \mathrm{dph}$ & $45 \mathrm{dph}$ & Juvenile \\
\hline LA-Hb $\alpha 1$ & 36 & 36 & 31 & 35 & 33 & 34 & 28 & 24 & 18 \\
\hline LA-Hba2 & 36 & 36 & 29 & 31 & 29 & 30 & 28 & 26 & 25 \\
\hline $\mathrm{MN}-H b \alpha 1$ & 35 & 36 & 36 & 35 & 34 & 35 & 35 & 33 & 27 \\
\hline $\mathrm{MN}-\mathrm{Hb} \alpha 2$ & ND & ND & 31 & 33 & 32 & 30 & 24 & 19 & 17 \\
\hline $\mathrm{MN}-H b \alpha 3$ & 35 & 35 & 23 & 25 & 23 & 25 & 23 & 24 & 28 \\
\hline $\mathrm{MN}-\mathrm{Hb} \alpha 4$ & 36 & 37 & 25 & 27 & 23 & 24 & 21 & 19 & 26 \\
\hline $\mathrm{MN}-\mathrm{Hb} \alpha 5$ & 36 & 36 & 24 & 25 & 24 & 25 & 22 & 21 & 29 \\
\hline LA- $H b \beta 1$ & 36 & ND & 26 & 27 & 24 & 23 & 20 & 18 & 17 \\
\hline $\mathrm{MN}-H b \beta 1$ & 36 & 37 & 33 & 35 & 35 & 35 & 33 & 32 & 30 \\
\hline $\mathrm{MN}-H b \beta 2$ & ND & ND & 35 & 36 & 35 & 35 & 28 & 23 & 19 \\
\hline $\mathrm{MN}-H b \beta 3$ & 36 & 37 & 36 & ND & ND & ND & ND & 36 & 36 \\
\hline $\mathrm{MN}-H b \beta 4$ & 37 & 34 & 22 & 23 & 21 & 23 & 21 & 20 & 21 \\
\hline $\mathrm{MN}-H b \beta 5$ & 33 & 35 & 22 & 23 & 22 & 24 & 23 & 26 & 29 \\
\hline $\mathrm{MN}-H b \beta 6$ & ND & 35 & 24 & 24 & 21 & 22 & 19 & 18 & 23 \\
\hline
\end{tabular}


Supplementary file 6. $\mathrm{Cq}$ (Quantification cycle) values of $\mathrm{Hb} \alpha$ and $\mathrm{Hb} \beta$ genes in different tissues of adult European sea bass. ND means Not Detected.

\begin{tabular}{|c|c|c|c|c|c|c|c|}
\hline Gene & Heart & Intestine & Head Kidney & Spleen & Gills & Liver & Brain \\
\hline LA-Hb $\alpha 1$ & 33 & 29 & 31 & 32 & 34 & 34 & 34 \\
\hline LA-Hba2 & 26 & 30 & 22 & 26 & 27 & 32 & 28 \\
\hline $\mathrm{MN}-H b \alpha 1$ & 19 & 22 & 12 & 17 & 19 & 24 & 20 \\
\hline $\mathrm{MN}-H b \alpha 2$ & 26 & 29 & 25 & 24 & 26 & 31 & 27 \\
\hline$M N-H b \alpha 3$ & 35 & 35 & 38 & ND & 37 & 38 & 35 \\
\hline $\mathrm{MN}-\mathrm{Hb} \alpha 4$ & 27 & 30 & 26 & 25 & 27 & 32 & 29 \\
\hline $\mathrm{MN}-H b \alpha 5$ & 34 & 33 & 36 & 35 & 36 & ND & 33 \\
\hline LA- $H b \beta 1$ & 29 & 26 & 29 & 27 & 29 & 34 & 30 \\
\hline $\mathrm{MN}-H b \beta 1$ & 19 & 22 & 12 & 17 & 18 & 24 & 20 \\
\hline $\mathrm{MN}-H b \beta 2$ & 30 & 34 & 28 & 27 & 30 & 33 & 30 \\
\hline $\mathrm{MN}-H b \beta 3$ & 29 & 33 & 22 & 27 & 29 & 34 & 30 \\
\hline $\mathrm{MN}-H b \beta 4$ & 32 & 36 & 30 & 30 & 33 & 37 & 33 \\
\hline $\mathrm{MN}-H b \beta 5$ & 36 & 36 & 33 & 36 & ND & ND & 36 \\
\hline $\mathrm{MN}-H b \beta 6$ & 30 & 30 & 32 & 34 & 32 & 36 & 30 \\
\hline
\end{tabular}

